\title{
Abdominal Aortic Aneurysm \\ Detection by Common Femoral Artery \\ Doppler Ultrasound Waveform Analysis
}

\section{Catherine E Wells}

\author{
Ph.D. Thesis
}

Cardiff University 
UMI Number: U585114

All rights reserved

\section{INFORMATION TO ALL USERS}

The quality of this reproduction is dependent upon the quality of the copy submitted.

In the unlikely event that the author did not send a complete manuscript and there are missing pages, these will be noted. Also, if material had to be removed, a note will indicate the deletion.

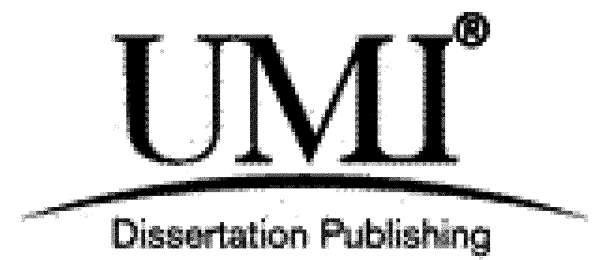

UMI U585114

Published by ProQuest LLC 2013. Copyright in the Dissertation held by the Author.

Microform Edition (c) ProQuest LLC.

All rights reserved. This work is protected against unauthorized copying under Title 17, United States Code.

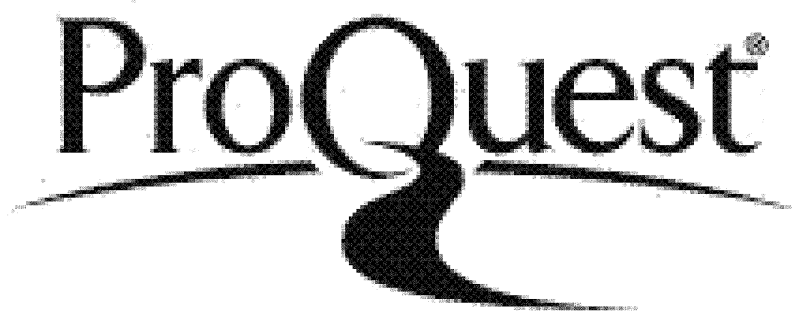

ProQuest LLC

789 East Eisenhower Parkway

P.O. Box 1346

Ann Arbor, MI 48106-1346 


\begin{tabular}{|l|l|}
\hline Candidate's ID number & 100027663 \\
\hline Candidate's Surname & Wells \\
\hline Candidate's Forenames & Catherine Elizabeth \\
\hline
\end{tabular}

\section{Declaration}

This work presented in this thesis has not already been accepted in substance for any degree and is not being concurrently submitted in candidature for any degree.

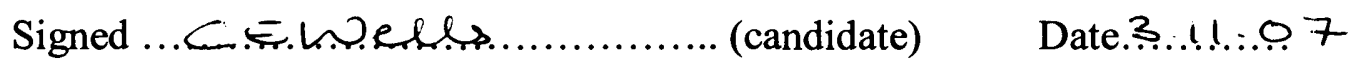

\section{Statement 1}

This thesis is being submitted in partial fulfilment of the requirements for the degree of Ph.D.

Signed ....

\section{Statement 2}

The thesis is the result of my own independent work/investigations, except where otherwise stated. Other sources are acknowledged where appropriate. A full bibliography is appended.

Signed ...F.E.t.e.ls .............. (candidate) Date.3...1.:.0.7

\section{Statement 3}

I hereby give my consent for my thesis, if accepted, to be available for photocopying and for inter-library loan, and for the title and summary to be made available to outside organisations.

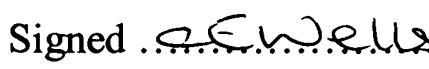
(candidate)

Date..3.11....77 


\section{Summary}

Abdominal Aortic Aneurysm (AAA) is a serious, life threatening condition. AAAs are often asymptomatic and many remain silent until rupture. If detected early, the condition can be monitored and electively repaired relatively safely. Arterial disease is readily and routinely assessed using Doppler ultrasound. The effect of occlusive arterial disease on Doppler waveforms is well documented and the disease state of the aorto-iliac segment can be estimated by examination of the common femoral artery (CFA) waveform. Theoretically, aneurysmal disease should alter the blood flow pattern due to the localised increase in vessel diameter. It has been noted during routine clinical examinations of CFA Doppler waveforms, that when certain features were observed, an AAA was often present. Reported studies examining lower limb Doppler waveforms and flows through aneurysm models support these observations.

The main aim of this research study was to determine if and how the Doppler waveform of the CFA is changed in the presence AAA and to utilise these changes for the early diagnosis of AAA. The interpretation of Doppler waveforms for the assessment of vascular disease requires a high level of skill and training and still remains subjective. An automated, objective detection method to detect AAA by analysis of the CFA Doppler waveform was achieved by implementing a MATLAB software based computer program to perform CFA waveform feature based analysis and determine whether or not an AAA is present. The second aim of the study was to predict the AAA size and presence of ILT using the program results.

For patients with no significant atherosclerotic disease, automated MATLAB based CFA waveform analysis provided $100 \%$ sensitivity and $73.3 \%$ specificity for AAA detection. Additional waveform features set up by atherosclerotic disease made it more difficult to separate the AAA and normal patients with significant atherosclerotic disease. It was not possible to adequately predict the physical characteristics of using the AAA detection program.

In conclusion, feature analysis of the CFA Doppler waveform may provide a promising, alternative method for AAA detection. AAA characterisation may be possible using different analysis methods. 


\section{Acknowledgements}

I would firstly like to thank my supervisors Professor Woodcock and Dr Neil Pugh for giving me the opportunity to undertake this $\mathrm{PhD}$ and for their support and advice over the past 6 years.

I would also like to thank Huntleigh Healthcare for providing the funding and technical support for the project. Special thanks to Dr Nigel Gough for his help and advice.

A big thank you to everyone in Doppler Ultrasound, especially to Declan, Rhys and Paul for their support.

Thank you to Andy for his support and encouragement throughout this project.

A special thank you to my Mum and Dad for their unending love, support and inspiration over the past 27 years. 


\section{Contents}

Research Overview.......................................................

Chapter 1: Introduction..................................................

1.1 Arterial Circulation...............................................

1.2 Lower Limb Arteries.............................................4

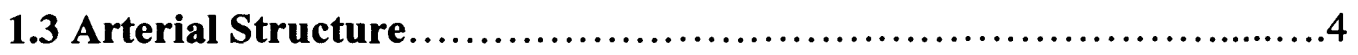

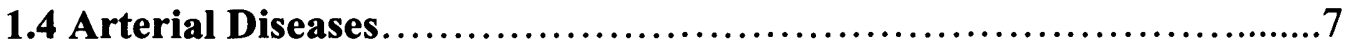

1.4.1 Atherosclerosis..........................................

1.4.2 Peripheral Arterial Disease...............................8

1.4.3 Aneurysmal Disease..................................9

1.5 Abdominal Aortic Aneurysm..................................11

1.5.1 Clinical Management of AAA .............................13

1.5.2 AAA Screening ........................................ 15

1.5.3 Aneurysms of the Distal Arteries.........................16

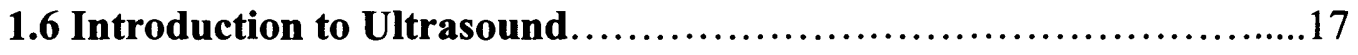

1.6.1 Ultrasound Generation....................................17

1.6.2 Medical Ultrasound.....................................18

1.6.3 B-Mode Ultrasound Imaging...........................20

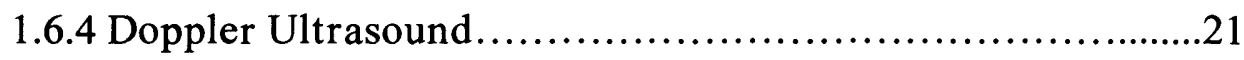

1.6.4.1 Spectral Doppler................................22

1.6.4.2 Continuous wave Doppler.......................22

1.6.4.3 Pulsed Wave Doppler............................23

1.6.4.4 Duplex Imaging.................................24 
1.6.4.5 Colour Flow Doppler Imaging......................24

1.6.4.6 Colour Flow Duplex Imaging.....................25

1.6.5 Doppler Ultrasound Assessment of Arterial Disease..........26

1.7 Arterial Haemodynamics...................................... 28

1.7.1 Blood Pressure and Impedance...........................28

1.7.2 Laminar Flow and Turbulence.............................29

1.7.3 Spectral Broadening....................................32

1.7.4 Doppler Waveform Shapes..............................32

1.7.5 Characteristic Waveform Shapes............................33

1.7.5.1 Occlusive Arterial Disease and Lower Limb

Arterial waveforms...............................35

1.7.5.2 Hyperaemic Waveform Changes...................37

1.8 Haemodynamics of AAA......................................... 38

1.8.1 Mechanical Fluid Dynamics Modelling of AAA................40

1.8.1.1 Physiological Effects of AAA Flow Patterns.......... 47

1.8.2 Numerical Fluid Dynamics Modelling.......................49

1.8.2.1 Effect of Intra-luminal Thrombus.....................51

1.8.3 Detection of AAA Flow Disturbances In Vivo.................53

1.9 Discussion and Conclusions...........................................

Chapter 2: Equipment and General Methods...............................61

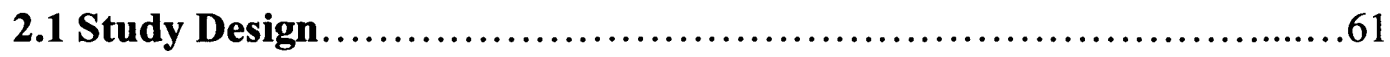

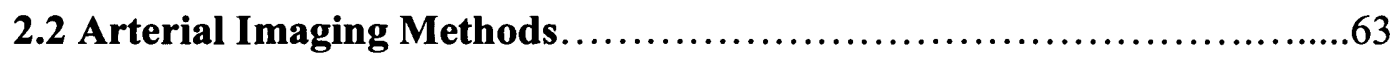

2.2.1 Toshiba Xario System..................................63 
2.2.2 AAA Scan Protocol........................................63

2.2.3 Lower Limb Arterial Scan Protocol.......................65

2.3 Waveform Measurement Methods................................66

2.3.1 Waveform Measurement Equipment......................66

2.3.2 Measurement Protocol......................................66

2.3.3 Comparison of Toshiba Xario and DopStudio Waveforms....67

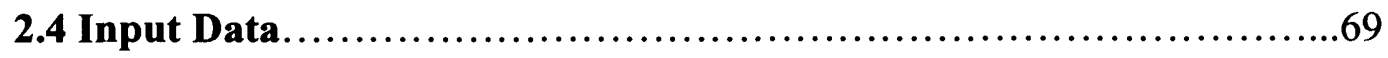

2.5 Data Summary ................................................. 70

Chapter 3: Visual Analysis of CFA Waveforms from AAA and Normal

Patients with No Significant Atherosclerotic Disease........................ 74

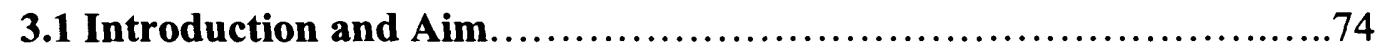

3.2 Method............................................................ 74

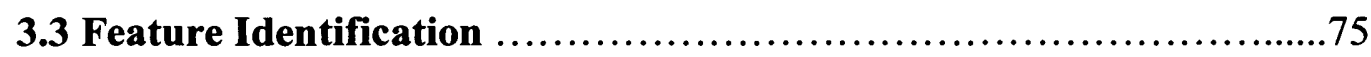

3.4 AAA Detection Using CFA Visual Feature Analysis.................80

3.4.1 Waveform Feature Results.............................81

3.4.2 Waveform Feature Results: Discussion.....................82

3.4.3 Individual Patient Scores..................................82

3.4.4 Individual Patient Score Results...........................82

3.4.5 Individual Patient Score Results: Discussion.................84

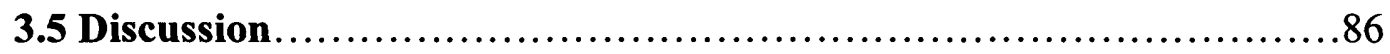


Chapter 4: AAA Detection by Objective Analysis of CFA waveforms in Patients with No Significant Atherosclerotic Disease.......................92

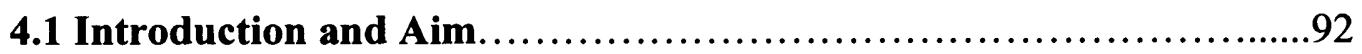

4.2 Method Overview................................................93

4.3 Initial Data Processing........................................94

4.3.1 Extraction of the Maximum Frequency Envelope............94

4.3.2 Identification of Individual Waveform Cycles..............96

4.3.2.1 Identification of the Systolic Peak..................96

4.3.3 Noise Reduction......................................98

4.3.3.1 Thresholding..................................99

4.3.3.2 Automatic Thresholding........................101

4.3.4 Power Level Normalisation................................103

4.4 Calculation of Waveform Parameters............................... 103

4.4.1 Maximum Frequency Envelope Calculation.................104

4.4.2 Minimum Frequency Envelope Calculation..................104

4.4.3 Bandwidth Envelope Calculation...........................105

4.4.4 Power Envelope Calculation.............................106

4.4.5 Systolic Peak Markers....................................107

4.4.6 Reverse Flow and Second Forward Flow Phase Markers.....107

4.5 Identification of Measures to Describe CFA Waveform Features.......109

4.5.1 Spectral Broadening Indices...............................110

4.5.2 Systolic Down Stroke ‘Spikes’ Index.........................117

4.5.3 Irregular Reverse Flow Indices...........................121

4.5.4 Simultaneous Forward and Reverse Flow Indices............130

4.5.5 Elongation Indices................................... 138 
4.6 AAA Detection Program: Combination of Indices to Discriminate

between AAA and Normal Patients............................... 141

4.7 AAA Detection Program Results................................... 148

4.7.1 AAA Detection Program Study Groups' Results...............148

4.7.1.1 AAA Detection Program Study Groups'

Results: Discussion....................................150

4.7.2 Response of AAA Detection Program to Blind Test Groups..151

4.7.2.1 AAA Detection Program Blind Test Groups'

Results

.159

4.7.2.2 AAA Detection Program Blind Test Groups'

Results: Discussion.

4.8 AAA Detection Program Performance for Patients with AAA and

Additional Aneurysmal Disease.................................... 162

4.8.1 AAA Detection Program Additional Aneurysm Group

Results......................................................169

4.8.2 AAA Detection Program Additional Aneurysm Group

Results: Discussion........................................170

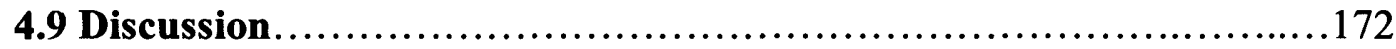

Chapter 5: Visual Inspection of CFA Waveforms from AAA and

No AAA Patients with Significant Atherosclerotic Disease......................175

5.1 Introduction and Aims............................................ 175

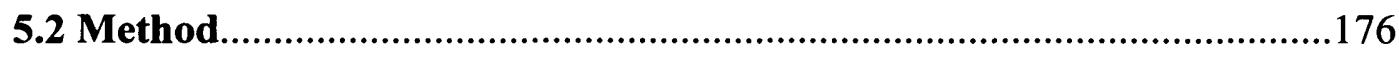

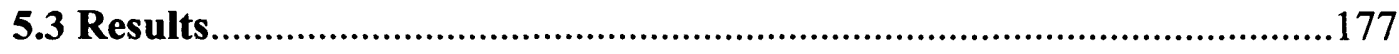


5.3.1 Visual Inspection of CFA Waveforms from

Patients with Significant Femoro-Popliteal Disease.

5.3.1.1 Feature Results...............................177

5.3.1.2 Description of Features.........................179

5.3.1.3 AAA Detection in Patients with Significant

Femoro-Popliteal Atherosclerotic Disease Using CFA

Visual Feature Analysis

5.3.2 Visual Inspection of CFA Waveforms from Patients with

Significant Proximal Atherosclerotic Disease.......................190

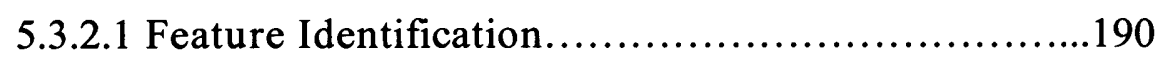

5.3.2.2 AAA Detection in Patients with Significant

Proximal Atherosclerotic Disease Using CFA Visual

Feature Analysis.....................................193

5.4 Discussion....................................................... 198

Chapter 6: Objective Analysis of CFA waveforms from AAA and No AAA Patients with Significant Atherosclerotic Disease using MATLAB software...............200

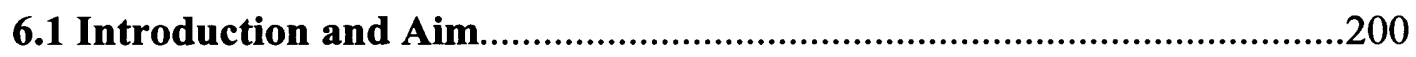

6.2 Performance of the AAA Detection Program for Patients with

Significant Femoro-Popliteal Atherosclerotic Disease...................201

6.2.1 Preliminary Tests.......................................201

6.2.2 Initial AAA Detection Program Performance Results..........202

6.2.3 Initial Program Results: Discussion.......................204 
6.3 Optimisation of the AAA Detection Program for Patients with

Significant Femoro-Popliteal Atherosclerotic Disease..................205

6.3.1 Maximum Systolic Down-Stroke Bandwidth Index in the Distal Disease Groups........................................206

6.3.2 Systolic Spike Index in the Distal Disease Groups............208

6.3.3 Reverse Power Density Index in the Distal Disease Groups...211

6.3.4 Reverse to Systolic Height Ratio Index in the Distal

Disease Groups............................................213

6.3.5 Integral of Reverse Power Under Systolic Upstroke

Index in the Distal Disease Groups..............................215

6.3.6 Integral of Reverse Power Under Systolic Down-Stroke

Index in the Distal Disease Groups..............................218

6.4 Disease AAA Detection Program.................................220

6.4.1 Disease AAA Detection Program Study Groups' Results.......220

6.4.2 Disease AAA Detection Program Study groups'

Results: Discussion.......................................222

6.5 Response of Disease AAA Detection Program to Blind Test Groups...224

6.5.1 Blind Test Groups' Results...............................231

6.5.2 Blind Test Groups' Results: Discussion ...................232

6.6 AAA Detection Program for Patients with Significant

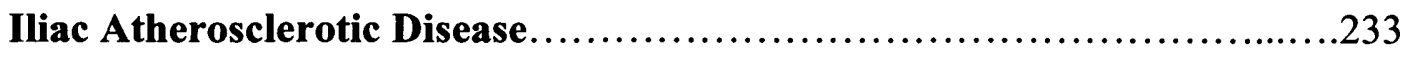

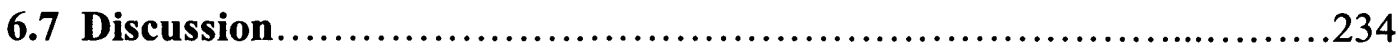


Chapter 7: Characterisation of AAA In Patients with No Significant

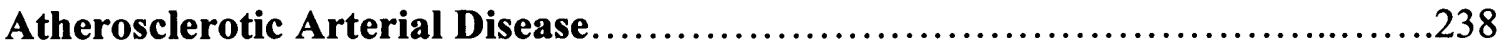

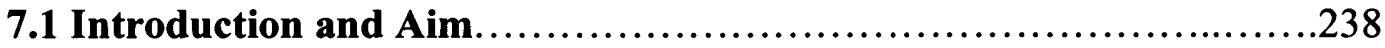

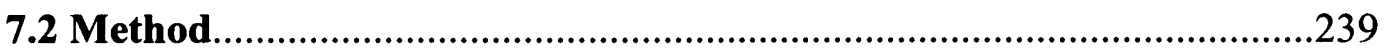

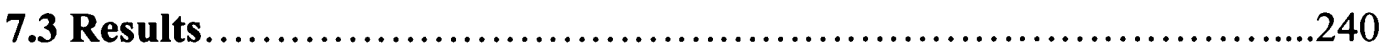

7.3.1 Investigation of Total Patient Scores with AAA Size and ILT

7.3.2 Investigation of Individual Index Scores for

AAA Size Characterisation........................................242

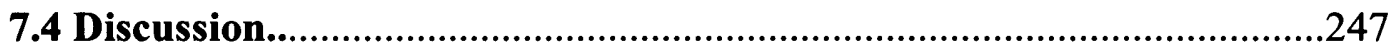

Chapter 8: Summary and Conclusions.......................................248

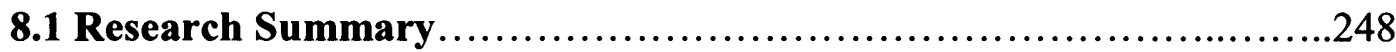

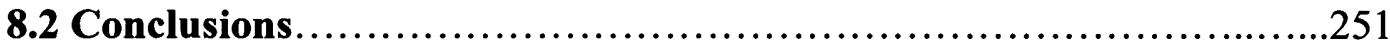

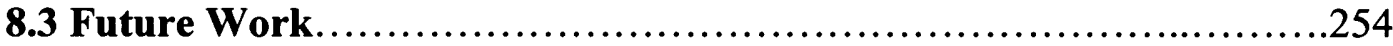

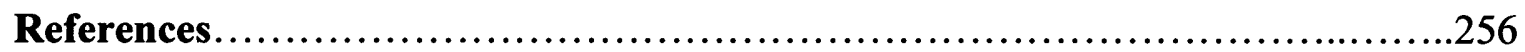

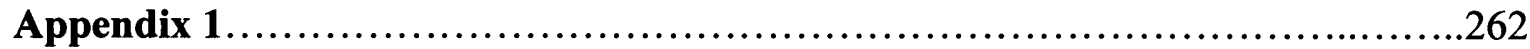

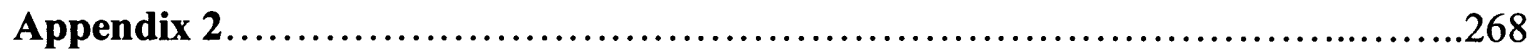

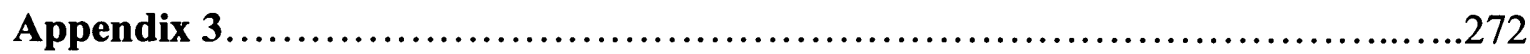

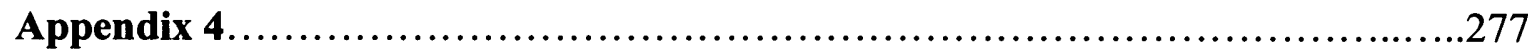

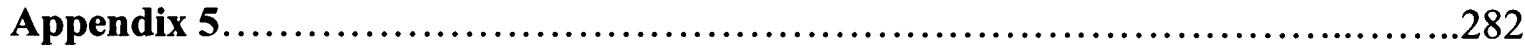




\section{$\underline{\text { Research Overview }}$}

Abdominal Aortic Aneurysm (AAA) is a potentially fatal condition which results in an estimated 10,000 deaths per year in the UK (Smith 1994). AAA is a progressive condition that often remains clinically silent for many years until the aneurysm is very large and at risk of rupture. Serious complications such as embolisation of intraluminal thrombus resulting in lower limb ischaemia with the possible need for amputation may be the first clinical indication of an AAA. Despite the seriousness of this condition, AAA often remains undiagnosed until rupture because there are no prior symptoms. Fifty percent of patients with a ruptured AAA die before reaching hospital and the chance of surviving emergency surgery is low. If detected early, the prognosis is much improved because the condition can be monitored and elective repair can be considered. AAAs that are diagnosed intact are often an incidental finding of physical examinations, x-rays or other scans performed for an unrelated medical condition. At present there is no national screening programme for AAA but studies have shown that ultrasound screening for AAA saves lives and is cost effective (Ashton, Buxton et al. 2002).

Arterial disease is readily and routinely assessed using colour flow duplex ultrasound scanning. The effect of occlusive arterial disease on the Doppler waveform is well documented and the disease state of the aorto-iliac segment can be estimated by examination of the common femoral artery waveform. The aim of this research study is to examine the haemodynamics of AAAs and try to determine if and how the Doppler waveform of the common femoral artery is changed in the presence of an AAA, and to utilise these changes for the early diagnosis of AAA. This work came 
about because the project supervisor Dr Neil Pugh who has over 20 years experience of vascular ultrasound noted during routine clinical work that when certain changes were observed in the CFA waveform, an AAA was often present. This is supported by similar observations noted in an early study by Humphries et al (Humphries, Hames et al. 1982). Therefore it may be feasible to detect AAA by analysis of the CFA waveform. The interpretation of Doppler waveforms for the assessment of vascular disease requires a high level of skill and training and still remains subjective. The overall aim of this research project was to develop an automatic, objective method to detect AAA by analysis of the CFA Doppler waveform. This was achieved by implementing a MATLAB software based computer program to perform CFA waveform analysis and determine whether or not an AAA is present based on the results. 


\section{Chapter 1: Introduction}

\section{$\underline{1.1 \text { Arterial Circulation }}$}

Blood is circulated around the body by the action of the heart and is transported through the arteries, veins and capillaries which make up the vascular system. Oxygenated blood is pumped from the ventricles of the heart into the aorta, the largest artery in the body. From the aorta, the large arteries branch to form the arterial supply to different regions of the body. These large arteries branch repeatedly to form many progressively smaller arteries to supply more peripheral tissues. The aorta is generally described in three main sections: The ascending aorta from which the coronary arteries branch to supply the cardiac muscle, the aortic arch from which the brachiocephalic, left common carotid and left subclavian arteries branch to supply the head and upper limbs and the descending aorta which supplies the rest of the body. The descending aorta is the longest section of the aorta and extends through the thorax and down through the abdomen to the superior margin of the pelvis. It is divided into the thoracic aorta lying within the thorax and the abdominal aorta which runs distally from the diaphragm. The aorta terminates at the level of the fifth lumbar vertebra where it divides into the right and left common iliac arteries to supply the pelvis and lower limbs. The branches of the thoracic aorta supply the thoracic organs including the lungs. The abdominal aorta has several major branches supplying the abdominal organs: the celiac trunk, superior and inferior mesenteric arteries branch to supply the stomach and intestines, the paired renal arteries branch to supply the kidneys, the gonadal arteries supply the gonads and the lumbar arteries supply the spine (Seeley, Stephens et al. 2000). 


\subsection{Lower Limb Arteries}

The lower limbs are supplied from the right and left common iliac arteries (CIAs) which bifurcate from the abdominal aorta just below the level of the navel. The common iliac arteries run bilaterally, and bifurcate in the pelvis to form the left and right internal iliac arteries which supply the pelvic region and the external iliac arteries, which continue distally to the groin to supply the legs. The lower limb arteries form a continuum but change name at the level of the groin and popliteal fossa. At groin level the external iliac artery becomes the common femoral artery (CFA). At the top of the leg the common femoral artery bifurcates into the profunda femoris artery (PFA) which supplies the thigh and the superficial femoral artery (SFA) which continues distally. The superficial femoral artery becomes the popliteal artery at the level of the popliteal fossa. The popliteal artery trifurcates into three main tibial arteries: The posterior tibial artery, anterior tibial artery and the peroneal artery below the level of the knee joint. The dorsalis pedis and plantar arteries run from these arteries onto the foot.

\subsection{Arterial Structure}

The regulation of blood flow to tissues is in part achieved by the function of the arteries. Arterial structure is intricate with adaptive characteristics allowing either dilatation or constriction in response to alterations in haemodynamic stresses and environmental conditions to maintain an optimal blood supply to tissues (Zarins, Xu et al. 2005). 
The arterial wall consists of three distinct layers or tunis. These are the tunica intima, tunica media and the tunica adventitia (Seeley, Stephens et al. 2000). The inner void through which the blood flows is called the patent artery lumen. The innermost layer called the tunica intima consists of a delicate basement membrane of connective tissue called the endothelium and a layer of elastic fibres called the internal elastic membrane. This membrane separates the tunica intima from the adjacent layer the tunica media. The tunica media extends from this internal elastic layer and consists of a matrix of closely packed and predominantly elastic fibres with smooth muscle and collagen fibres interwoven. The elastin fibres provide extensibility, allowing for compliance and recoil of the artery wall in response to the fluctuation in the blood pressure during the cardiac cycle, and their arrangement uniformly distributes the tensile stress on the wall. The collagen fibres have a high elastic modulus and provide tensile strength to limit distension at high pressures. The tunica media extends to the outer layer called the tunica adventitia. This outer layer is relatively thin and is composed of collageneous connective tissue, the outer layers of which merge with the surrounding connective tissue to tether the artery ((Seeley, Stephens et al. 2000), (Zarins, Xu et al. 2005)).

The arteries form a continuum from the aorta to the smallest distal branches, but the relative thickness of each layer and the composition of elastic tissue and muscle within the arterial wall gradually changes through the vascular tree. Arteries are classified as either elastic or muscular depending on their composition. The large arteries proximal to and including the aorta are elastic. Their walls contain a large amount of elastic tissue and a comparatively small proportion of muscle. As the arteries branch away from the aorta, decreasing in size, there is a gradual transition in 
the wall composition from predominantly elastic to predominantly muscular wall composition. Therefore the smaller, more distal arteries are classed as muscular (Seeley, Stephens et al. 2000). Muscular arteries have relatively thick walls that contain relatively less collagen and elastin and more smooth muscle allowing for constriction or dilatation to facilitate rapid changes in diameter. In the larger elastic arteries the layers of elastic fibres called lamalae are tightly packed with alternating layers of smooth muscle and collagen fibres interwoven. The number of lamellar units and the intima media thickness are closely related to the tangential tension experienced by the wall and the luminal radius (Zarins, Xu et al. 2005).

The average tension per lamellar unit tends to be constant throughout mammalian species. The arterial radius increases with increasing mammal size with corresponding increases in the intima medial thickness and lamellar units. However, this relationship does not hold for the human abdominal aorta. In humans the abdominal aorta contains fewer lamellar units than would be expected for the load borne. This may explain the relatively high incidence of abdominal aortic aneurysms in humans. The intima media of the proximal thoracic aorta contains a larger proportion elastin and a lower proportion of collagen than the abdominal aorta or more distal elastic arteries. The proximal aorta is more compliant than the abdominal aorta but is also more prone to tear. Studies have suggested that failure of elastin and collagen in the abdominal aorta is a major factor in aneurysm development (Curci, Baxter et al. 2005; Zarins, Xu et al. 2005). 


\subsection{Arterial Diseases}

The two main types of disease affecting the arteries can be categorised into those which decrease the lumen diameter and those by which the arterial diameter is increased. Although this study is concerned with the detection of aneurysmal disease of the abdominal aorta, occlusive arterial disease and its effect on the blood flow must also be considered.

\subsubsection{Atherosclerosis}

Arteriosclerosis is a general term used to describe a number of conditions in which the walls of the arteries thicken and harden (Solomon, Schmidt et al. 1990). The most common form of arteriosclerosis is atherosclerosis derived form the Greek words 'athero' meaning gruel and 'sclerosis' meaning hardness. Atherosclerosis mainly affects the larger arteries of the body: The coronary arteries of the heart, the carotid arteries supplying the brain and also the peripheral arteries supplying the limbs. It is the main cause of cardiovascular disease and is the leading cause of death in the United States (Solomon, Schmidt et al. 1990). It is a slow, progressive disease involving the deposition of lipids, cholesterol, calcium and other substances of the blood to form plaque within the lining of the arterial wall. Atherosclerotic plaque formation reduces the elasticity of the arterial wall and gradually narrows the lumen impeding the flow of blood. Lesions which cause narrowings are called stenoses. The severity of disease is characterised by the degree of narrowing and is usually referred to as the percentage of the vessel diameter which is blocked. A $50 \%$ stenosis refers to a narrowing of $50 \%$. As plaque formation progresses the stenosis level increases until 
eventually the artery is blocked completely. This is called an occlusion. Both stenoses and occlusions can significantly reduce the blood supply to distal tissues.

\subsubsection{Peripheral Arterial Disease}

Peripheral arterial disease (PAD) broadly describes diseases effecting the arteries supplying the limbs. This is commonly atherosclerotic plaque effecting the aorto-iliac, femoral and popliteal arteries. The atherosclerotic lesions reduce the blood supply distally, therefore the symptoms of PAD depend on the location and severity of the disease. The most common symptom is intermittent claudication where no pain is felt at rest but cramp like pain is experienced in the leg muscles, most commonly the calf muscles on exertion. With advanced aorto-iliac disease pain may be felt at rest and the function of pelvic organs may also be affected. The severity of disease is classified according to the affect it has on the blood flow: Its haemodynamic significance. It is generally accepted that stenotic lesions causing less than $30-40 \%$ narrowing are not clinically significant as there is no real reduction to the distal flow volume. $40-50 \%$ stenoses are generally classed as moderately haemodynamically significant. Stenoses greater than $70 \%$ dramatically impede the flow of blood. Complete arterial occlusion is the endpoint at which the artery is completely blocked and the blood supply to distal tissues is reliant upon collateral circulation. Collateral arteries are the distributing branches of large and medium sized arteries that enlarge when a stenosis or occlusion develops to maintain a supply of blood distally.

Studies indicate the prevalence of PAD of the lower limbs to be around $12 \%$. The disease is progressive and the prevalence has been shown to increase with age from 
around $7 \%$ for those aged $60-69$ years old to around $23 \%$ for those aged over 80 years old (Fowkes, Housley et al. 1991; Ostchega, Paulose-Ram et al. 2007).

\subsubsection{Aneurysmal Disease}

An aneurysm is a gross, permanent, localised dilatation of a section of an artery. An enlargement greater than twice the size of the proximal artery is generally classed as aneurysmal. Arterial aneurysms are differentiated into three main forms as shown in Figure 1.1: Fusiform where the dilation is symmetrical, saccular where the dilation is at one side of the artery and fusiform/saccular where all sides are dilated but nonsymmetrically. In all cases intra-luminal thrombus (ILT) may form on the artery wall within the dilated region.

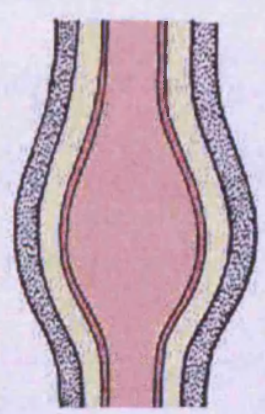

Fusiform aneurysm

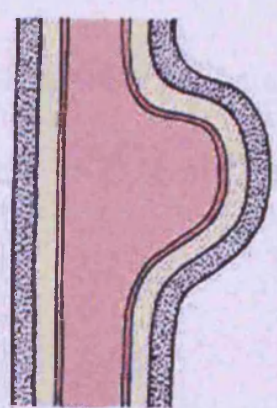

Saccular aneurysm

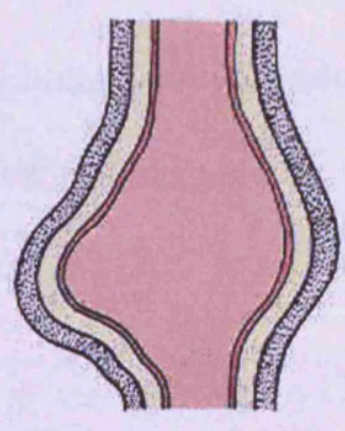

Fusiform/saccular aneurysm

Figure 1.1: Fusiform, saccular and fusiform/saccular aneurysms (Hennerici and Neuerburg-Heusler 1998)

The majority of aneurysms develop slowly over many years. They are often clinically silent until late in their natural history, and may remain undetected until dissection, embolisation of ILT or rupture occurs (Curci, Baxter et al. 2005). 
Structural weakness and mechanical and haemodynamic forces are the basic factors underlying aneurysm development. The aetiology of arterial aneurysms is not entirely understood and involves a complex interaction of genetic, local and systemic factors. The development of aneurysmal disease involves changes to all three layers of the arterial wall, the tunica intima, tunica media and the tunica adventitia. Collagen and elastin are the major structural proteins within the arterial wall matrix. There are several pathologies which appear to be central to the initiation and progression of aneurysmal disease: Matrix changes within the tunica media and tunica adventitia with damage to the elastic intima, chronic inflammation, and the depletion of cells responsible for matrix repair. The interrelation between these pathologies means that these changes are progressive and self-propagating once established. The changes may be brought about by mechanical injury, congenital defects, infection and inflammatory conditions. Congenital defects in proteins namely elastin and collagen within the arterial wall matrix can predispose to aneurysm development. Two such inherited syndromes being Marfan syndrome and Ehlers-Danlos syndrome. In both these conditions collagen formation is defective causing faulty elastic tissue (Curci, Baxter et al. 2005). 


\subsection{Abdominal Aortic Aneurysm}

The normal diameter of the abdominal aorta in the average adult ranges from around $1.2 \mathrm{~cm}$ to $2.0 \mathrm{~cm}$. It is generally agreed that an aortic diameter exceeding $3 \mathrm{~cm}$ is classed as aneurysmal. The most common type of aortic aneurysm is an abdominal aortic aneurysm (AAA), which occurs in the portion of the aorta lying below the renal arteries as illustrated in Figure 1.2. Aortic aneurysms may also occur in the thoracic cavity.

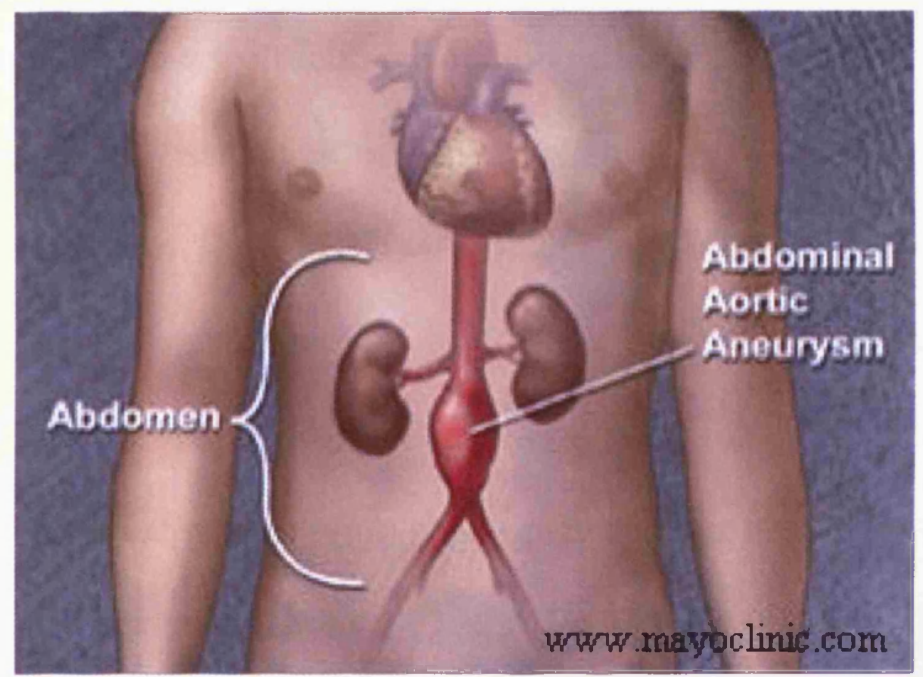

Figure 1.2: Abdominal Aortic Aneurysm

Most AAAs are fusiform/saccular in shape. The dilation in the anterior direction is usually greater than in the posterior direction which is limited by the spine. The shape of the aneurysm has clinical implications due to differences in prognosis, treatment and surgical procedures associated with the different forms (Hennerici and Neuerburg-Heusler 1998). 
AAA is a potentially lethal condition because if the aneurysm becomes very large it can rupture, and this is often fatal due to severe internal bleeding. It is estimated that AAAs are responsible for up to 10,000 deaths per year, approximately $1.7 \%$ of all deaths in England and Wales (Smith 1994). A serious complication of AAA is arterial embolism from the formation of ILT within the aneurysm. Embolic occlusions result in ischaemia of the tissues (commonly the feet and toes) supplied by the artery. Unless the emboli are removed by surgery or thrombolitic drugs, or bypassed, the occlusion may result in necrosis (tissue death) and amputation.

Common symptoms of AAA include abdominal pain, back pain, and some patients may experience a pulsating feeling in the abdomen. The symptoms are non-specific to AAA and do not usually occur until the aneurysm is very large. Patients can often have an aortic aneurysm for many years before any symptoms develop. In many cases, an aortic aneurysm will remain clinically silent until it ruptures, and around $50 \%$ of patients with a ruptured AAA die before reaching hospital (Ashton, Buxton et al. 2002). The likelihood of surviving emergency rupture repair is between $30-70 \%$ and the overall mortality rate from ruptured AAA is between $80-90 \%$ (Smith 1994). Aortic aneurysms that are diagnosed prior to rupture are usually found incidentally on physical examinations and chest or abdominal $\mathrm{x}$-rays performed for an unrelated condition. In some cases an acute occlusion of the lower limb arteries caused by emobolisation of ILT may be the first symptom of an AAA.

It is difficult to determine the prevalence of AAA because only those found incidentally or those that rupture are diagnosed, but screening studies have shown prevalences of up to $7.6 \%$ in men and $1.3 \%$ in women (Scott, Wilson et al. 1995; 
Scott, Bridgewater et al. 2002). The age-prevalence curve for women is shifted by about 10 years for women compared to men (Scott, Bridgewater et al. 2002). A study by Semmens et al indicates that on average women presenting with ruptured AAA are 6 years older than men (Semmens, Norman et al. 2000).

\subsubsection{Clinical Management of AAA}

Once an AAA has been detected, the patient will usually have an ultrasound scan to locate and measure the aneurysm. Ultrasound can be used to image the aorta in $99 \%$ of people (Ashton, Buxton et al. 2002). Colour flow duplex systems which allow simultaneous display of Doppler blood flow information and greyscale B-mode images are currently the principal type of equipment used for clinical diagnosis of peripheral vascular disease (Hennerici and Neuerburg-Heusler 1998). The reliability of colour flow duplex scanning for routine surveillance of abdominal aortic aneurysms is well recognised (Yehuda G. Wolf 2000) and is now considered the accepted non-invasive method in detecting and assessing AAAs (Hennerici and Neuerburg-Heusler 1998). At UHW, all patients with a diagnosed AAA undergo an ultrasound scan every six or twelve months in a surveillance programme to monitor the growth of the aneurysm. The maximum diameter of the aneurysm is measured in both transverse and longitudinal planes. The extent of the aneurysm and its shape can normally be estimated. The extent of any ILT and the size of the remaining patent lumen is also measured. 
The treatment for AAA is surgical or endovascular repair. In these procedures the aneurysmal section of the artery is replaced by a synthetic graft. For elective aneurysm repair, the risk of rupture must justify the surgery considering the potential complications and expense. The survival of emergency aneurysm repair following rupture is low and although the surgical risk is still high, studies show the survival rate of elective surgery is much greater. The mortality rate for elective surgery is 2$6 \%$ (Ashton, Buxton et al. 2002) and therefore elective resection is considered when the risk of rupture exceeds the surgical risk. The risk of rupture increases with aneurysm diameter and becomes substantial when the diameter exceeds $5-6 \mathrm{~cm}$. The UK Small Aneurysm Trial indicated that for small aneurysms $(<5.5 \mathrm{~cm}$ diameter $)$, the mortality rate due to risk of rupture in patients under ultrasound surveillance was far lower than the mortality rate in patients who underwent early elective surgery (Pretre R 1998). The vascular surgeons at the University Hospital of Wales (UHW) will consider patients for elective aneurysm repair if the aneurysm diameter exceeds $5.5 \mathrm{~cm}$ and therefore is considered at risk from rupture. Ultrasound scanning has advantages over other techniques such as CT (computed x-ray tomography) and MR (magnetic resonance) angiography in terms of wider availability, lower cost, and it does not involve ionising radiation. However, in addition to measuring the aneurysm size, the shape, location and extension (for example, into the renal and iliac arteries) must be determined. Conventional, CT and MR angiography can provide this information which is not usually attainable with duplex scanning. This information is valuable to the surgeon in the selection of surgical procedure for resection (Baur G M 1978). Therefore, an angiographic procedure is often performed prior to elective repair. 


\subsubsection{AAA Screening}

At present there is no national screening programme for AAA. Several studies have shown that screening of the male population over the age of 65 years for AAA significantly reduces the mortality rate from the condition (Scott, Wilson et al. 1995; Heather, Poskitt et al. 2000; Ashton, Buxton et al. 2002; Wilmink, Quick et al. 2003). The Multicentre Aneurysm Screening Study (MASS) (Ashton, Buxton et al. 2002; Greenhalgh and Powell 2002) presented reliable evidence of benefit from ultrasound screening for AAA. The advantage of screening is that if AAAs are detected prior to rupture, the condition can be monitored and may be electively repaired if clinically viable. The study suggested a $51 \%$ reduction of ruptured AAAs and showed that over four years there was a $53 \%$ mortality reduction in men who were screened for AAA. In addition to the increased survival for elective repair, the cost is almost half that of emergency repair, and therefore AAA screening was shown to be cost effective.

Although screening for AAA using conventional ultrasound techniques is cost effective, duplex scanning is hospital based, relatively expensive (approx. $£ 80$ per NHS scan) and requires a skilled operator to perform the investigation. The aim of this research study was to develop an alternative technique to diagnose AAA that is cheaper and requires reduced operator skill. 


\subsubsection{Aneurysms of the Distal Arteries}

Aneurysmal disease can also affect the larger arteries distal to the aorta supplying the lower limbs, commonly the iliac arteries at the bifurcation of the aorta and the popliteal arteries behind the knee. In general for these arteries, a diameter exceeding $1.5 \mathrm{~cm}$ is considered aneurysmal. 


\subsection{Introduction to Ultrasound}

Sound is audible to the human ear within the frequency range $20 \mathrm{~Hz}-20 \mathrm{KHz}$. Frequencies higher than about $20 \mathrm{kHz}$ are inaudible and beyond this level the sound wave is known as ultrasound. A sound wave is a longitudinal pressure wave which passes through a medium at a fixed speed (Fish 1990). The rate at which particles in the medium vibrate in the disturbance is the frequency of the sound. The speed at which an ultrasound wave is transmitted through a medium is dependant upon the density and compressibility of the medium in the relationship $c=\sqrt{k / \rho}\left(\mathrm{ms}^{-1}\right)$ where $\mathrm{c}$ is the propagation speed, $\mathrm{k}$ is the elastic modulus $\left(\mathrm{kgms}^{-1}\right)$ and $\rho$ is the density $\left(\mathrm{kgm}^{-3}\right)$. The acoustic impedance $(Z)$ of a material is a measure of the response of the particles in a medium to a given pressure and is given by $Z=\rho c\left(\mathrm{kgm}^{-2} \mathrm{~s}^{-1}\right)$ (Fish 1990). Acoustic impedances within the human body cover a wide range from $430 \mathrm{kgm}^{-2} \mathrm{~s}^{-1}$ for air to $6.47 \times 10^{6} \mathrm{kgm}^{-2} \mathrm{~s}^{-1}$ for bone. Soft tissues such as kidney and liver have acoustic impedances of $1.64 \times 10^{6} \mathrm{kgm}^{-2} \mathrm{~s}^{-1}$ and $1.66 \times 10^{6} \mathrm{kgm}^{-2} \mathrm{~s}^{-1}$ respectively and the acoustic impedance of blood is $1.67 \times 10^{6} \mathrm{kgm}^{-2} \mathrm{~s}^{-1}$ (Hoskins, Thrush et al. 2003).

\section{$\underline{\text { 1.6.1 Ultrasound Generation }}$}

Ultrasound is generated using a piezoelectric transducer which converts an electrical signal into a mechanical pressure wave. Piezoelectric materials expand or contract when a positive or negative voltage is applied across them, and generate a positive or negative voltage when compressed or stretched. The piezoelectric transducer can be used to generate an ultrasound pressure wave by applying an AC voltage with a frequency equal to that of the required ultrasound wave. The same or a second 
piezoelectric crystal is used to detect incoming (reflected) ultrasound waves by converting the returning pressure waves back into a measurable voltage.

\section{$\underline{1.6 .2}$ Medical Ultrasound}

Ultrasound first came into routine clinical use in the early 1970's (Cosgrove D 1993). These early systems provided real-time greyscale 'B-mode' images of structures within the body. Until this time, aortic aneurysms were diagnosed by physical examination and confirmed by conventional $x$-rays or arteriography. The development of Doppler systems in the same decade enabled blood flow within the body to be studied.

As ultrasound waves are transmitted through the body they are reflected at boundaries between tissues of different acoustic impedance with the amount of transmission and reflection dependent upon the difference in acoustic impedance across the boundary. The type of reflection is dependant on the size of the reflecting interface with respect to the wavelength of the ultrasound which is related to the frequency and speed of the

ultrasound by $\lambda=c / f$. For structures with dimension several wavelengths larger than the wavelength of the ultrasound, the wave is simply reflected as described above. For structures smaller than the wavelength of the ultrasound the wave is scattered in all directions by a phenomenon known as Rayleigh scattering. In the human body the soft tissues and organs have very similar acoustic impedance therefore the magnitude of the reflected signals from organ boundaries is very small, to the order of $1 / 10$ to $1 / 100$ of the incident wave intensity. Rayleigh scattering from blood cells is to the order of $1 / 1000$ of that from an organ boundary. 
To transmit ultrasound into the body the transducer is placed in contact with the skin surface with coupling gel to eliminate the air gap which would strongly reflect the ultrasound and reduce transmission into the body. As ultrasound propagates through tissue, its intensity decreases as energy is transferred into the tissue structure via a number of mechanisms. The absorption of ultrasound energy increases with frequency and the upper limit for ultrasound imaging is about $20 \mathrm{MHz}$. Structures lying deep within the body can be difficult to image and generally the maximum depth at which a reasonable clinical image can be obtained is $15-20 \mathrm{~cm}$. Structures lying beneath bone or gas often cannot be imaged as the majority of ultrasound is reflected at the boundary rather than transmitted through to the underlying tissue. These factors can pose problems when imaging the aorta in obese patients or in cases where there is excessive bowel gas present.

There are different types of transducers with a range of operating frequencies available for different imaging applications. For medical imaging applications sound waves in the range $1-15 \mathrm{MHz}$ are used. Because the penetration of ultrasound is strongly dependent on ultrasound frequency, lower frequencies are used to image deep lying structures such as the aorta. The array of transducer elements can be arranged convexly to provide a wider field of view. The type of transducer used to image structures such as the aorta is a low frequency curvi-linear transducer operating at around $4 \mathrm{Mhz}$. 


\subsubsection{B-Mode Ultrasound Imaging}

A 2-D B-mode ultrasound image is a grey-scale image representing tissue and organ boundaries in a slice through the body. B-mode stands for Brightness-mode because all pixels in the image are intensity modulated, which means the brightness of each pixel is proportional to the intensity of the ultrasound reflected from the corresponding structure within the body.

In medical B-mode imaging the ultrasound is transmitted into the body in a series of short pulses typically $0.2 \mu \mathrm{s}$ in duration. The depth of a reflecting structure in the body can be calculated by measuring the time lapse between the transmission of the ultrasound pulse and the reception of the reflected wave. To determine the lateral position of a structure in a 2-D image a multi-element transducer with an array of several hundred individual transducer elements is used. Small groups of elements are fired in sequence at a rate called the pulse repetition frequency (PRF) to generate a few hundred 'scan lines' in a plane through the body. The location of each pixel in the resulting 2-D image corresponds to a depth calculated from the assumed tissue velocity and a lateral position calculated from the PRF. An ultrasound image or 'frame' is generated once all the transducer elements in the array have been fired. Modern ultrasound scanners generate around 30 frames per second enabling 'real time' imaging of structures within the body. The size of structures within the image can be measured using on-screen callipers. The distance between the callipers is calculated using the timing information stored within the image matrix. A B-mode image of an AAA with calliper measurements of its size is shown in Figure 1.3. 


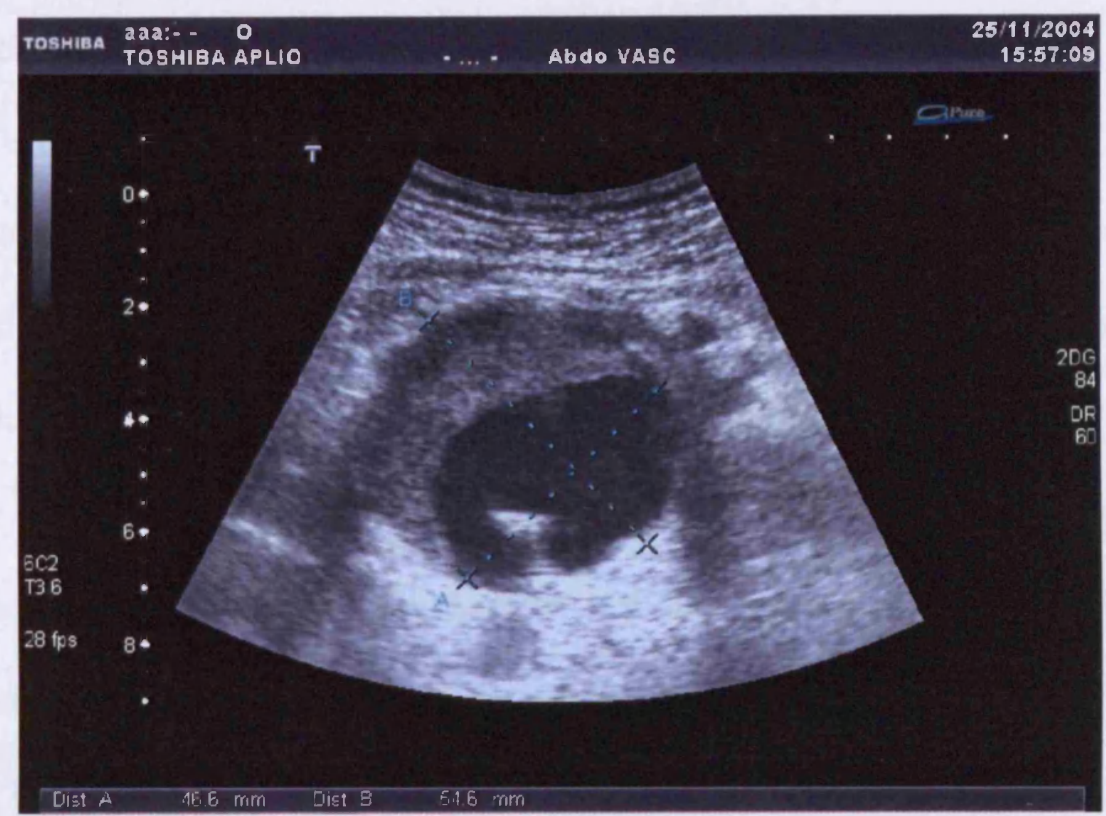

Figure 1.3: B-mode image of an AAA with calliper measurements of size

\subsubsection{Doppler Ultrasound}

Doppler ultrasound techniques utilise the Doppler effect and enable the motion of tissue and blood flow in the body to assessed. The Doppler effect is the change in observed frequency of a sound wave compared to the emitted frequency, which occurs due to the relative motion between the observer and the source of the sound wave. The frequency change is known as the Doppler Shift frequency. By this principle ultrasound can be used to assess blood flow by measuring the frequency shift of the ultrasound scattered from moving blood. The Doppler Shift Frequency $f_{d}$ is dependent upon the velocity of the moving blood $v$, the frequency of the transmitted ultrasound $f_{t}$, and the speed of ultrasound through tissue $c$, in the relationship: $f_{d}=\frac{2 v f_{t} \cos \theta}{c}$ where $\theta$ is the angle subtended between the ultrasound beam and the direction of blood flow (angle of insonation). 


\subsubsection{Spectral Doppler}

Conventionally, Doppler Shift signals are analysed by their spectral content. The most common display of the Doppler signal is in the form of a Doppler spectrogram. This is a graphical representation in which time is displayed along the horizontal axis, frequency shift is displayed along the vertical axis and the power at a particular time and frequency is displayed as the intensity of the corresponding pixel. In systems where the insonation angle can be estimated, the blood flow velocity can be calculated using the Doppler Equation and a graph of blood flow velocity versus time can be displayed. Clinically useful information is extracted from the spectral shape and parameters of the velocity waveform or power spectrum. An example of a Doppler velocity waveform measured from the common femoral artery (CFA) is shown in Figure 1.4.

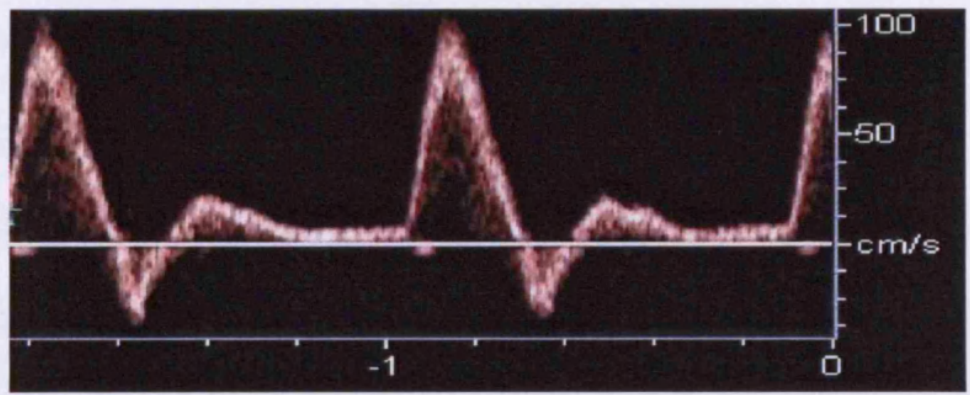

Figure 1.4: Doppler velocity waveform measured from the CFA

\subsubsection{Continuous Wave Doppler}

In the continuous wave Doppler technique a continuous wave of ultrasound is emitted from a transducer and the returning reflected wave is received by a second transducer within the same probe head. The Doppler shift signals are measured from any 
movement along the path of the ultrasound beam. The disadvantage of continuous wave Doppler is that the noise level in the signals can be high because Doppler shift signals from movement anywhere along the beam path are measured in addition to the shifts from blood flow in the vessel of interest. For example in the assessment of blood flow in the common femoral artery, Doppler shifts from blood flow in the underlying common femoral vein or other smaller blood vessels within the path of the beam may also be measured. In most vascular applications high pass filtering is implemented to reduce the noise in the signal from tissue movement. The advantage of the continuous wave Doppler technique is that it is relatively simple, with hand held devices available. The technique is useful for measuring blood flow in large arteries from which the magnitude of the signal is sufficient to overcome noise.

\subsubsection{Pulsed Wave Doppler}

In pulsed wave Doppler techniques the ultrasound wave is not transmitted continuously but as a series of short, typically $1-2 \mu$ s pulses. This enables the Doppler signal to be sampled from a selected depth within the body, for example within a particular artery. The depth at which the Doppler signal is measured is set by the time at which the signal is sampled after pulse transmission. The rate at which the individual pulses are fired is called the pulse repetition frequency (PRF). In accordance with sampling theory, the PRF must be at least twice the maximum Doppler shift frequency to be measured to avoid misinterpretation of the signals called signal aliasing. The pulsed wave Doppler technique is more involved but has the advantage that the signal is much cleaner because only Doppler shift signals from the region of interest are measured with signals from the surrounding tissue and other 
blood vessels eliminated. To reduce the noise even further, pulsed Doppler systems often employ high pass filtering to eliminate signals arising from the movement of the blood vessel wall.

\subsubsection{Duplex Imaging}

Duplex imaging combines B-mode imaging and pulsed wave Doppler measurements. The transducer is similar to a B-mode imaging transducer but with a small group of the transducer elements utilised to transmit and detect pulsed Doppler signals. The remaining elements employ the B-mode imaging. This technique allows the blood vessel to be imaged and the depth at which the Doppler signal is sampled is set by positioning an on-screen cursor called the 'Doppler gate'. The width of the Doppler gate can be altered to change the range of the depth within which the shifts are sampled. A second on screen 'angle' cursor within the Doppler gate can be aligned with the orientation of the vessel to give a measure of the insonation angle with the blood flow so that blood flow velocity can be calculated using the Doppler equation. This technique is clinically useful because the disease state of an artery can be imaged and the Doppler gate can be positioned to determine the haemodynamic significance of the disease.

\subsubsection{Colour Flow Doppler Imaging}

Colour flow Doppler imaging is a technique in which the Doppler shift signals are mapped onto a B-mode ultrasound image to give a visual representation of blood flow through vessels. The Doppler shift signals are colour coded often in red and blue to 
indicate flow away from (negative Doppler shift) or towards (positive Doppler Shift) the transducer respectively.

\subsubsection{Colour Flow Duplex Imaging}

Most modern ultrasound scanners can perform colour flow duplex (triplex) imaging. This is the simultaneous implementation of B-mode, colour flow mapping and pulsed wave Doppler. In this technique the Doppler gate can be positioned not only within the artery at the site of disease but at the point of maximum velocity indicated by the hue of the colour in the flow map. The Doppler angle cursor can be aligned with the flow to enable calculation of the blood flow velocity. A colour flow duplex image of the CFA is shown in Figure 1.5.

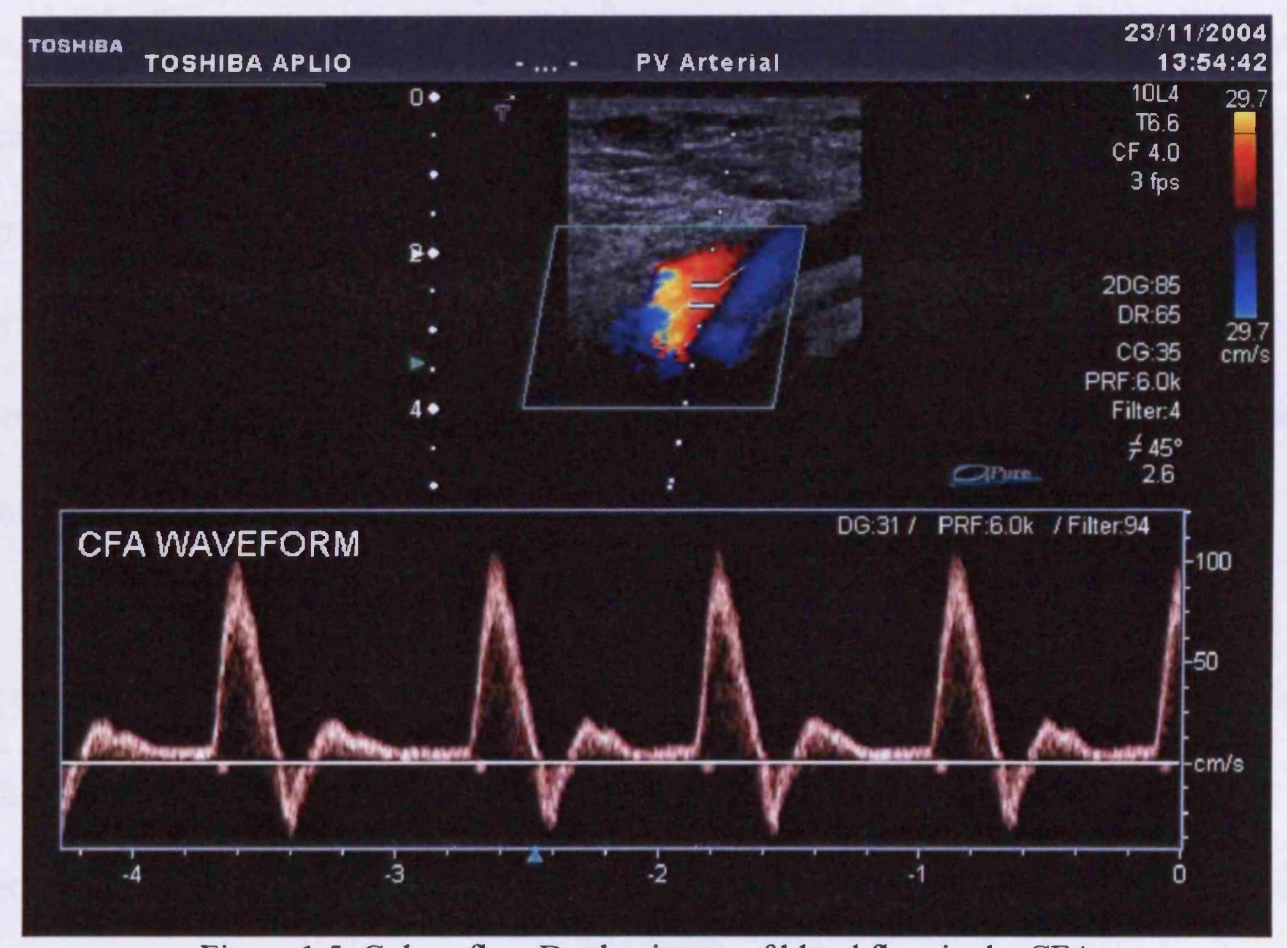

Figure 1.5: Colour flow Duplex image of blood flow in the CFA 


\subsubsection{Doppler Ultrasound Assessment of Arterial Disease}

Arterial disease is readily and routinely assessed using colour and spectral Doppler ultrasound which provide 'real-time' measurements of blood flow velocity. In the assessment of arterial disease the B-mode images, colour flow images and Doppler spectrograms are analysed visually to facilitate a diagnosis. Visual analysis of B-mode and colour flow images provide information about disease within the region of measurement. In the B-mode image, mural calcification, plaque, thrombus or aneurysms can be visualised. The superposition of the colour flow map can provide information as to the haemodynamic effects of the disease. To an experienced, trained eye the colour flow map can used to identify phasic, disturbed or damped flow. Different velocities are displayed as different colour hues and very high velocities cause aliasing of the colour.

Spectral Doppler is diagnostically very useful as it provides information about the haemodynamic effect of disease within the measurement region but can also indicate the presence and severity of proximal or distal disease. Measurement of blood flow velocities derived from the Doppler frequency spectrum give an estimate of the degree of narrowing caused by the local disease. Visual analysis of the Doppler waveform shape can indicate the presence of proximal or distal disease.

Analysis of the Doppler spectrogram is subjective and requires an experienced practitioner. The effects of atherosclerotic occlusive arterial disease on Doppler flow patterns (discussed in Section 1.7.5.1) are well documented. Visual assessment of waveform shape and quality can provide a qualitative analysis of disease state, for 
example observation of a damped waveform measured from the femoral artery indicates the presence of proximal disease. There are a number of parameters which can be extracted from a spectrogram which provide more quantitative analysis, for example the resistivity index (RI), pulsatility index (PI) and the Laplace damping factor ( $\delta)$ (Evans, McDicken et al. 1989). 


\subsection{Arterial Haemodynamics}

Arterial blood flow is determined by the arterial anatomy, the physical characteristics of blood, the regulatory mechanisms controlling the heart and blood vessels and the pathological state of the blood vessels. Interrelationships between blood flow, pressure and flow impedance through vessels are critical to the function of the vascular system (Seeley, Stephens et al. 2000). Arteries are not ideal, straight, smooth, rigid vessels, but bend and branch often with irregularities to their surface. To complicate matters blood flow is not steady but pulsatile due to the action of the heart. Hence, the laws of fluid dynamics in normal rigid tubes do not strictly apply but are useful in the explanation and prediction of haemodynamic behaviour.

\subsubsection{Blood Pressure and Impedance}

Blood flow is largely dependent upon pressure. The pumping of the ventricles creates blood pressure, the force exerted by blood on the wall of blood vessels. The maximum arterial pressure occurs when the left ventricle of the heart contracts and is called the systolic pressure. The minimum pressure occurs when the left ventricle is relaxed and is called the diastolic pressure. Blood flows when there is a pressure gradient along the length of the vessel and the greater the pressure difference the greater the rate of flow. The blood flow resulting from a pressure gradient is opposed by an impedance to blood flow in the distal vascular bed. The relationship between flow, pressure and resistance in a section of a vessel (under steady state conditions) is described by Poiseuille's law: flow $=\frac{\left(P_{1}-P_{2}\right) r^{4}}{8 v l}$ where $P_{1}$ is the pressure at the inlet to the 
section, $P_{2}$ is the pressure at the outlet of the section, $r$ is the radius of the vessel, $v$ is the viscosity of the fluid and $l$ is the length of the vessel. The blood pressure exerts a force $(F)$ on the arterial wall which is governed by Laplace's law: $F=D \times P$ where $D$ is the arterial diameter and $P$ is the blood pressure (Seeley, Stephens et al. 2000). Under this law, with constant pressure, the force exerted on the vessel wall increases with increased vessel diameter. This fact is of particular importance for aneurysmal disease. As the aneurysm diameter increases, the force exerted on the weakened wall increases, therefore as aneurysms enlarge, the risk of rupture increases (Sumner and Zierler 2005).

\subsubsection{Laminar Flow and Turbulence}

Laminar flow describes steady flow through a long straight, smooth vessel. Under these conditions blood behaves as if it is composed of an infinitely large number of concentric layers. The outermost layer experiences drag as it moves against the vessel wall. This layer in turn exerts a drag force on the next adjacent layer which exerts a drag on the next layer and so forth. This results in a series of concentric layers with the more central layers moving progressively faster than those towards the vessel wall creating a sheared velocity profile (Seeley, Stephens et al. 2000; Sumner and Zierler 2005).

The velocity profile of steady flow entering a vessel is flat with all layers moving at the same velocity. As the flow moves along the vessel the profile becomes blunted and then increasingly parabolic along the vessel length until the velocity gradient

extends to the centre of the vessel. The entrance length required to develop a 
parabolic profile is dependent upon the vessel radius and a dimensionless quantity called the Reynolds ( $\mathrm{Re})$ number in the relationship $L=k r R e$ ( $k$ is approximately constant for $R e>50$ ). $R e$ is dependant upon the inertial and viscous forces within the fluid. According to this relationship, the entrance length of many larger arteries approaches the length of the artery and it has been shown that the aorta has a blunted velocity profile (Sumner and Zierler 2005).

Laminar flow breaks down and becomes turbulent when the blood flow velocity exceeds a critical value corresponding to an $R e$ value of approximately 2000 or when there is an abrupt change in vessel diameter, the vessel bends sharply or the surface becomes irregular (Sumner and Zierler 2005). Under normal physiological and pathological conditions the $R e$ value in peripheral arteries is well below the critical value. However, arteries within the circulatory system are not ideal, straight, smooth vessels but bend, and branch often with irregularities to their surface and abrupt changes in diameter in the presence of occlusive arterial or aneurysmal disease. These features disrupt the normal laminar flow pattern and therefore the pattern of blood flow in the arterial system is often classified as disturbed rather than turbulent or laminar. At bifurcations and areas where the vessel curves, the velocity profile becomes skewed. Any narrowing in an artery disrupts the normal laminar flow pattern as illustrated in Figure 1.6.

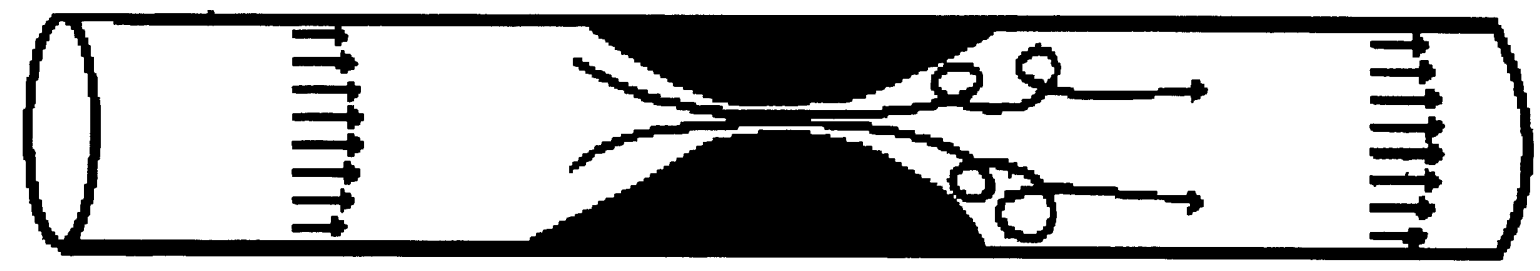

Figure 1.6: Flow disruption at a tight stenosis (based on Cosgrove D 1993). 
The extent to which flow is disrupted is proportional to the severity of the stenosis. The magnitude of the blood flow velocities are greatly increased through the narrowing and the degree of stenosis can be estimated from the peak systolic velocity. In the region of flow disruption just beyond the stenosis where the velocity vectors of the velocity profile are no longer parallel, the multiple frequencies produce a phenomenon called spectral broadening. Beyond a tight stenosis the laminar flow is most disturbed in a region of flow separation just beyond the narrowing. In flow separation there is a region of flow in which the velocity vectors have a different orientation to the main body of flow, for example flow reversal adjacent to the vessel wall. Within aneurysms the axial flow stream is separated from the flow within the bulge where velocities are low and flow reversal occurs as illustrated in Figure 1.7.

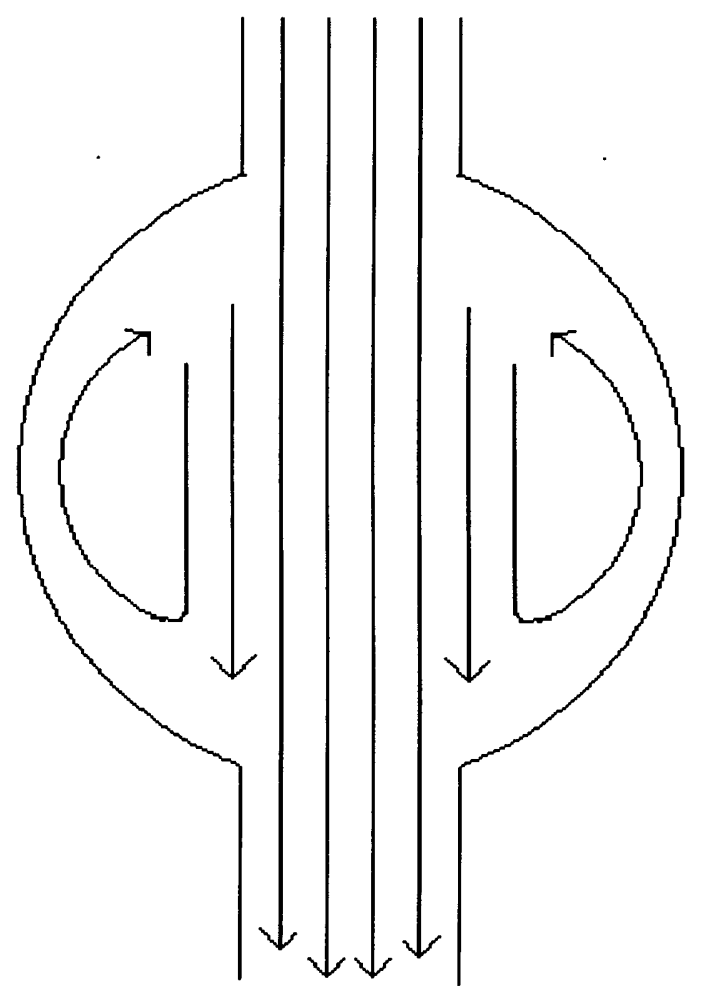

Figure 1.7: Flow reversal within an AAA (based on Sumner and Zierler 2005) 


\subsubsection{Spectral Broadening}

The maximum velocity of flow through an artery under laminar conditions occurs at the centre of the vessel. To make a measurement of this velocity using a duplex or colour flow duplex machine the Doppler gate is placed at the centre of the vessel using the on-screen cursor and the angle cursor is aligned with the flow direction. Because the Doppler gate has a finite width a range of velocities around the peak is sampled. This range is displayed as a band of frequencies (or velocities) on the Doppler spectrogram. Therefore the Doppler waveform is not a narrow line but has a width depending on the range of velocities sampled within the gate at a point in time. This is called spectral broadening. Under steady flow situations in the absence of disease the level of spectral broadening or 'band-width' of the signal is relatively low. Flow becomes increasingly disturbed in the presence of disease due to irregularities on the vessel wall. Increased velocities and the level of spectral broadening can be an indication to the level of disease present. In the continuous wave Doppler technique, signals are sampled throughout the beam path resulting in an intrinsically spectrally broad signal.

\subsubsection{Doppler Waveform Shapes}

The shape of the Doppler waveform within an artery is dependent on the inflow, the structure and composition of the local artery, its capacity for elastic recoil and dilatation, and the distal impedance of the vascular tree. The inflow is dependant upon the function of the heart and the disease state of the proximal vascular tree. The impedance to flow generally increases moving down the arterial tree from the aorta. 
When the blood flow meets high impedance at the peripheral vascular bed part of the pressure wave is transmitted into the capillaries and part is reflected back up the arterial tree. The fraction of the reflected pressure wave depends on the magnitude of the peripheral impedance. Reflection of pressure occurs at any point along the arterial tree where the artery branches, bifurcates or narrows and under normal pathological and physiological conditions the shape of the Doppler waveform in an artery is in part determined by the impedance of the vascular bed it supplies. Therefore, each different artery within the body has a characteristic Doppler waveform which is dependent on the vessel size and the local, proximal and distal impedance to flow (Sumner and Zierler 2005).

\subsubsection{Characteristic Waveform Shapes}

In the normal aorta and peripheral arteries of the lower limbs the vascular bed has high impedance resulting in a 'high impedance' waveform. In the normal aorta the Doppler waveform shape is described as triphasic as there are three distinct flow phases as shown in Figure 1.8.

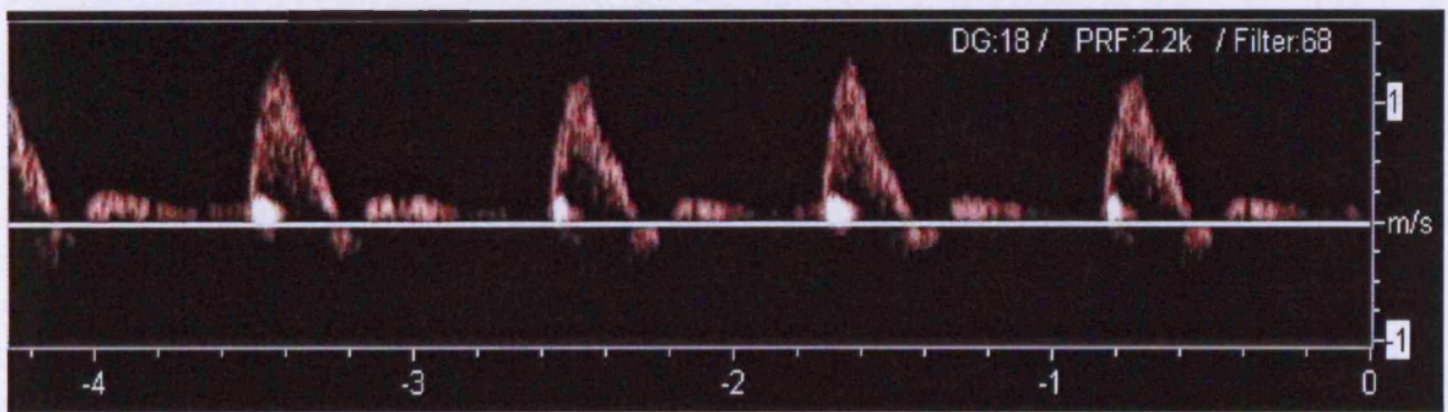

Figure 1.8: Doppler spectrogram of triphasic flow in normal aorta. 
During systole there is a forward, or antegrade flow phase as blood is propelled distally. This first peak is referred to as the systolic peak because it results from the systolic phase of the cardiac cycle. During early diastole, because the vascular bed is high impedance, the reflected pressure wave is relatively large resulting in an overall reverse, or retrograde movement of flow. In the Doppler spectrogram this is referred to as the reverse flow phase. In late diastole there is a second period of antegrade flow as blood is again propelled distally by elastic recoil of the artery wall. This peak is referred to as the second forward flow phase. In the common femoral artery (CFA) the waveform is also triphasic with both forward and reverse flow components due to cardiac output and elastic recoil of the artery. The shape of the waveform is characterised as high impedance due to the distal impedance of the vascular tree.

In the internal carotid artery which supplies the brain the impedance of the vascular bed is relatively low resulting in a 'low impedance' waveform. The reflected wave is relatively small and causes only a transient reduction in the forward flow during diastole but the flow remains antegrade throughout the cycle to maintain high volume blood flow to the brain as shown in Figure 1.9.

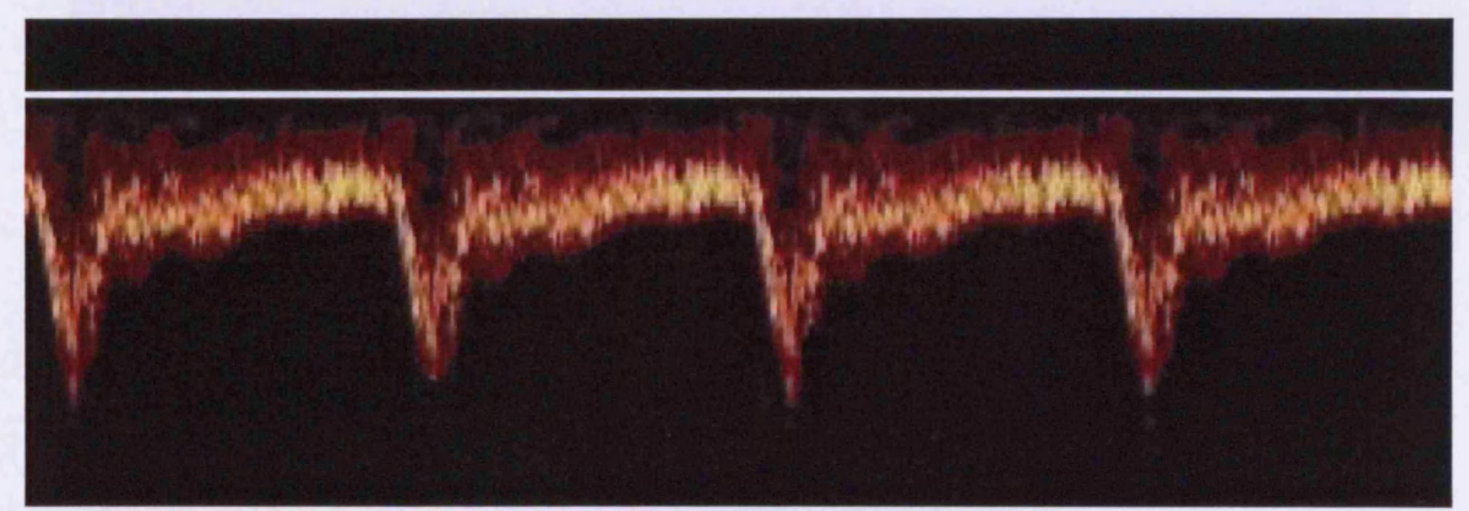

Figure 1.9: Internal carotid artery Doppler waveform. 


\subsubsection{Occlusive Arterial Disease and Lower Limb Arterial Waveforms}

The characteristic Doppler waveform in an artery is changed in the presence of local, distal or proximal disease. General hardening of the arterial wall reduces its elastic recoil capacity. This causes subtle changes to the waveform shape. The pulsatility of the waveform may be reduced and in effect the waveform becomes damped. This damping effect increases with the severity of the arterial disease and the second and third flow phases may eventually be lost. An arterial occlusion or stenosis, can significantly alter the waveform shape and spectral broadening both proximal and distal to the site of disease. A highly stenotic or occlusive lesion causes increased impedance. As compensation, in addition to collateral development, the vessels within the distal vascular bed dilate, reducing their impedance to try to maintain blood flow to the affected tissue. Proximal to an occlusive segment or significant stenosis the reflected pressure wave adds to the forward flow of systole, typically resulting in an upwards deflection or 'shoulder' on the downward slope of the Doppler waveform as shown in Figure 1.10.

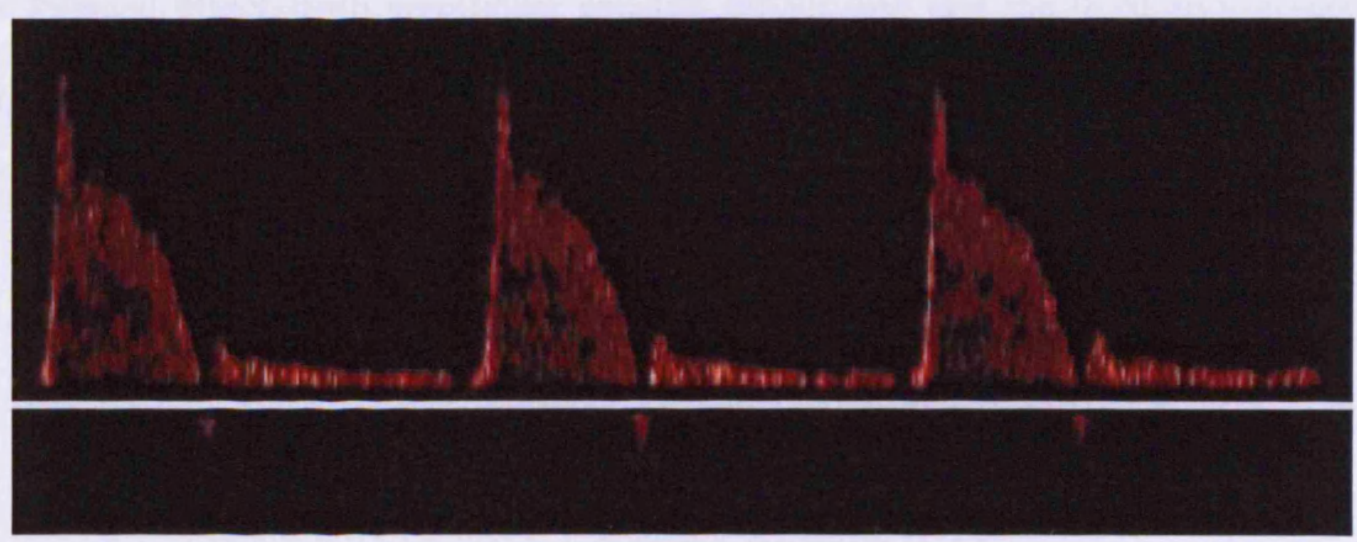

Figure 1.10: Shoulder on CFA waveform due to SFA occlusion 
Distal to an occlusion the pressure is greatly reduced and the amplitude of the waveform drops. The pulsatility in the waveform is diminished, the systolic peak becomes rounded, during diastole the reverse flow component is lost and the flow approaches the baseline more gradually as shown in Figure 1.11.

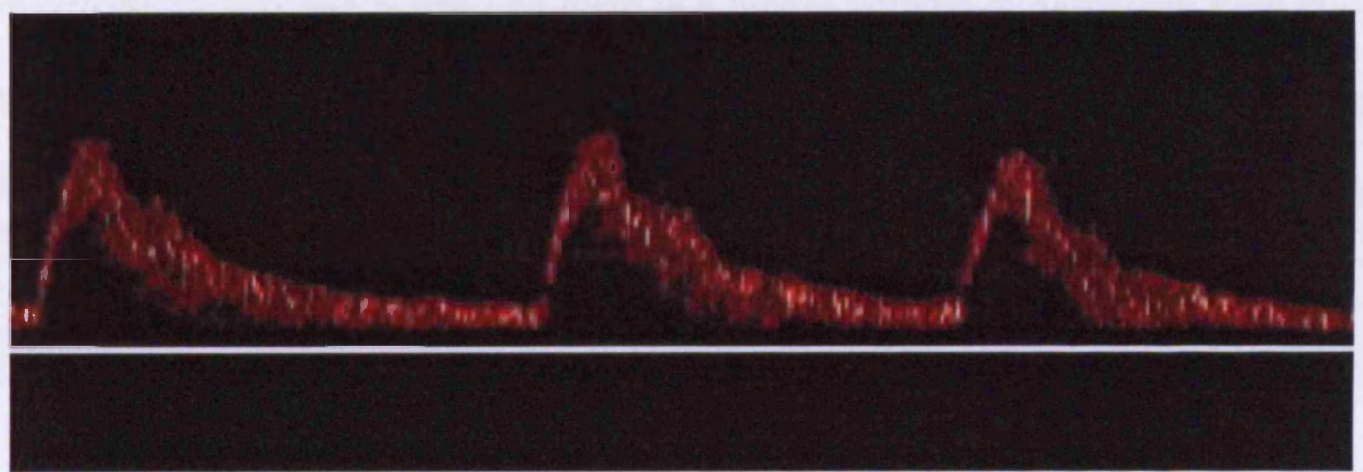

Figure 1.11: Damped flow distal to SFA occlusion

The extent of damping caused by stenotic lesions depends on the severity of the narrowing. With a minor to moderate stenosis, the alteration to the proximal and distal waveforms may be quite subtle. A significant stenosis will produce a more pronounced effect, with significant spectral broadening and the third and/or second phases of flow may be lost distally. 


\subsubsection{Hyperaemic Waveform Changes}

Doppler waveform shapes are altered by constriction or dilatation of the vascular bed in response to changes in environmental or systemic conditions. Dilatation of the vascular bed in response to an increased demand on blood flow reduces the impedance of the vascular bed and the waveform shape becomes lower impedance. The reverse flow phase is lost and there is a higher volume of antegrade flow during diastole. Such waveforms are described as hyperaemic. The triphasic waveform observed at rest in a healthy CFA can become hyperaemic for example upon exercise or in response to temperature changes. 


\subsection{Haemodynamics of AAA}

Within an aneurysm the local impedance and pressure is reduced due to the abrupt increase in vessel diameter. Therefore, AAA is likely to change the normal aortic flow pattern but it has not been possible with current imaging modalities to investigate the effects in vivo. Visualisation of Doppler flow patterns within AAAs is more difficult than in the more superficial peripheral vessels. The sensitivity and resolution of colour and spectral Doppler ultrasound is poor at the depth of the aorta, and the assessment of aorto-iliac disease using ultrasound techniques is known to be poor. Observation of AAA flows using colour flow duplex imaging is limited due to the thickness of overlying tissue and bowel gas. The flow can sometimes be imaged in cases where the AAA is particularly large or superficial. In these patients, Doppler colour flow maps may provide a crude representation of the flow. When flow can be imaged a characteristic swirling pattern with simultaneous forward and reverse flow is often observed indicating a level of flow disturbance. However, colour flow imaging does not provide any detailed information about AAA flow patterns. The colour-flow duplex image of flow in a $7.3 \mathrm{~cm}$ AAA is shown in Figure 1.12. The swirling pattern in the flow is crudely indicated by the red and blue regions of the colour-flow map. Doppler waveforms are very difficult to measure from within AAAs using duplex or colour flow duplex modes due to movement of the aorta with breathing and poor penetration through overlying tissue and bowel gas, resulting in weak, noisy signals. Therefore it is not possible to adequately examine AAA flows with current ultrasound techniques. 


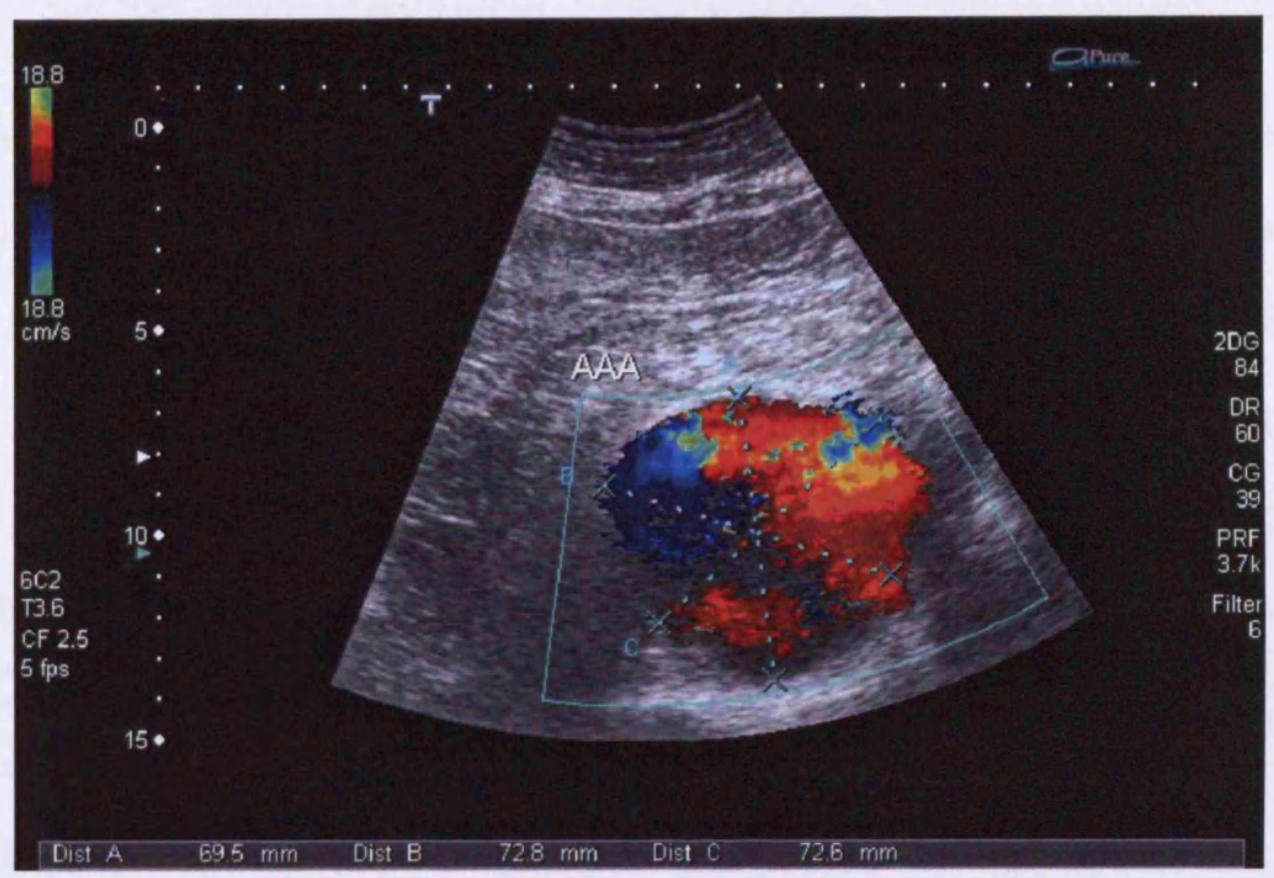

Figure 1.12: Colour flow duplex image showing flow through large AAA.

Theoretically, the presence of an aneurysm should also affect the flow patterns in distal vessels. Colour flow duplex imaging of the flow patterns distal to the AAA in the common iliac and external iliac vessels is again very difficult due to poor penetration, poor beam-vessel angle and tissue movement. The CFA is generally the most proximal artery supplying the lower limbs from which adequate Doppler information can be measured.

Because it has not been possible to investigate AAA flow patterns with current imaging techniques, recent studies into the haemodynamics of AAA have relied on numerical (computer) and mechanical fluid dynamics modelling. 


\subsubsection{Mechanical Fluid Dynamics Modelling of AAA}

Mechanical fluid dynamics modelling of flows within AAAs involves an AAA model, which is usually rigid, incorporated into a flow unit containing a blood substitute fluid. The descending aorta is modelled as a straight cylindrical tube. The flow pattern entering the AAA model is controlled by a pump and valve system. In early studies the flow patterns within the AAA models were measured using colour flow Doppler. In later studies an imaging technique called laser Doppler anemometry has been used to obtain more detailed measurements.

In early studies, Peattie and Schrader (Schrader, Peattie et al. 1992; Peattie, Schrader et al. 1994) used colour flow Doppler imaging to study the flow of tap water mixed with bone charcoal powder through a rigid fusiform AAA model. Colour Doppler imaging crudely reproduced the flow patterns within the model. At low flow rates the flow through the model remained steady, at higher flow rates $(\operatorname{Re}>2000)$ turbulence was observed as regions of retrograde flow, which fluctuated haphazardly in size and shape. However, the resolution of the colour flow Doppler images was not high enough to observe the detail of the flow patterns. In a later study by Peattie et al (Peattie, Asbury et al. 1996), the colour flow Doppler visualisation of the flow was supported by the more detailed laser Doppler anemometry measurements. Steady flows at a range of flow rates were passed through a number of different sized AAA models to examine the effect of AAA size on the flow. The instantaneous local flow velocities were qualitatively evaluated from the laser Doppler measurements. The critical $R e$ values for the onset of intermittent and non-intermittent turbulence were determined by increasing the flow rate into the model until turbulence was detected. 
This was repeated for a number of different sized AAA models. Three distinct flow regimes were observed within all the models. Below $R e=1800$, the core flow through the aneurysm remained laminar, surrounded by slowly recirculating fluid in the bulge. Above $R e=2400$ the flow was fully turbulent. Between $R e=1800$ and 2400 the flow was intermittently turbulent with the duration of turbulence increasing with increasing Reynolds number. For moderate and large AAAs $(4.3 \mathrm{~cm}$ and $6.3 \mathrm{~cm}$ diameter), at $R e=500$ the flow was laminar with slowly recirculating vortices surrounding the core flow. The most rapid retrograde velocities occurred in the distal half of the models. The flow was turbulent at $R e=2600$. The turbulence spread outwardly as it travelled through the aneurysm until it was reconstricted at the distal end of the aneurysm model by the exit tube. The critical conditions for turbulence were found to be a decreasing function of AAA diameter. Larger AAA models became turbulent at lower $R e$ values than smaller AAAs, and larger AAAs were more likely to develop higher intensity turbulent flow patterns. The largest models produced velocity fluctuations as great as $40 \%$ of the time averaged centre line velocities. In all cases, the strongest turbulence was found in the distal half of the AAA and the turbulent bursts increased in size as they travelled through the AAA model. These early studies of steady flows through AAA models suggest that AAAs are likely to disrupt normal aortic flow patterns. It is very difficult to reproduce truly physiologically representative flow patterns but the following later studies went on to investigate more realistic flow patterns.

Yip and Yu have published several papers on their work investigating flows through rigid aortic aneurysm models using laser Doppler anemometry. Yu (Yu 2000) examined steady flow through an axis-symmetric (fusiform) AAA model and 
observed the formation of a recirculating vortex occupying the entire circular bulge with the core located towards the distal end. The same model was then used to examine the effect of more realistic pulsatile flows. In this case, Yu observed vortex formation towards the proximal end of the bulge during the early acceleration phase of the flow cycle. This vortex increased in strength and moved towards the distal end of the AAA model during the deceleration phase. The vortex rapidly decayed during the subsequent acceleration phase. The study was conduced using two different size AAA models of $4 \mathrm{~cm}$ and $8 \mathrm{~cm}$ diameter to simulate early and late stage aneurysms. For steady flow, bulge dimension did not largely affect the flow characteristics. For pulsatile flow the vortex strength was greater in the larger aneurysm.

In a later study (Yip and Yu 2001), a similar experimental set up was used to investigate the transition to turbulence within aneurysm models for more realistic oscillatory flows. Axis-symmetric (fusiform) AAA models of $32 \mathrm{~mm}$ and $64 \mathrm{~mm}$ diameter and $80 \mathrm{~mm}$ and $100 \mathrm{~mm}$ respectively in length were used to model early and late stage AAAs. The input flows were based on physiological measurements of aortic flow taken by Moore et al. The reverse components of the oscillatory flow were removed by a valve system. The experiments were performed for two different simulated flow conditions: fully laminar flow $(R e<500)$, and transitional flow $(500<\operatorname{Re}<1310)$. For the advanced stage AAA model, turbulence was observed within the AAA for fully laminar $(R e<500)$ aortic (input) flow. Recirculating vortices were formed at the proximal end of the model as the flow entered the bulge. These increased in size and moved towards the distal end, separating from the wall. The strength of the vortices was almost completely dissipated on arrival at the distal end. The onset of turbulence occurred at the distal neck immediately after the flow began 
to decelerate. The turbulence was not sustained, because the flow was relaminarised when the flow began to accelerate in the subsequent flow cycle. This flow regime measured by Yip and Yu is illustrated in Figure 1.13, which shows the development of flow through the aneurysm during a complete flow cycle.
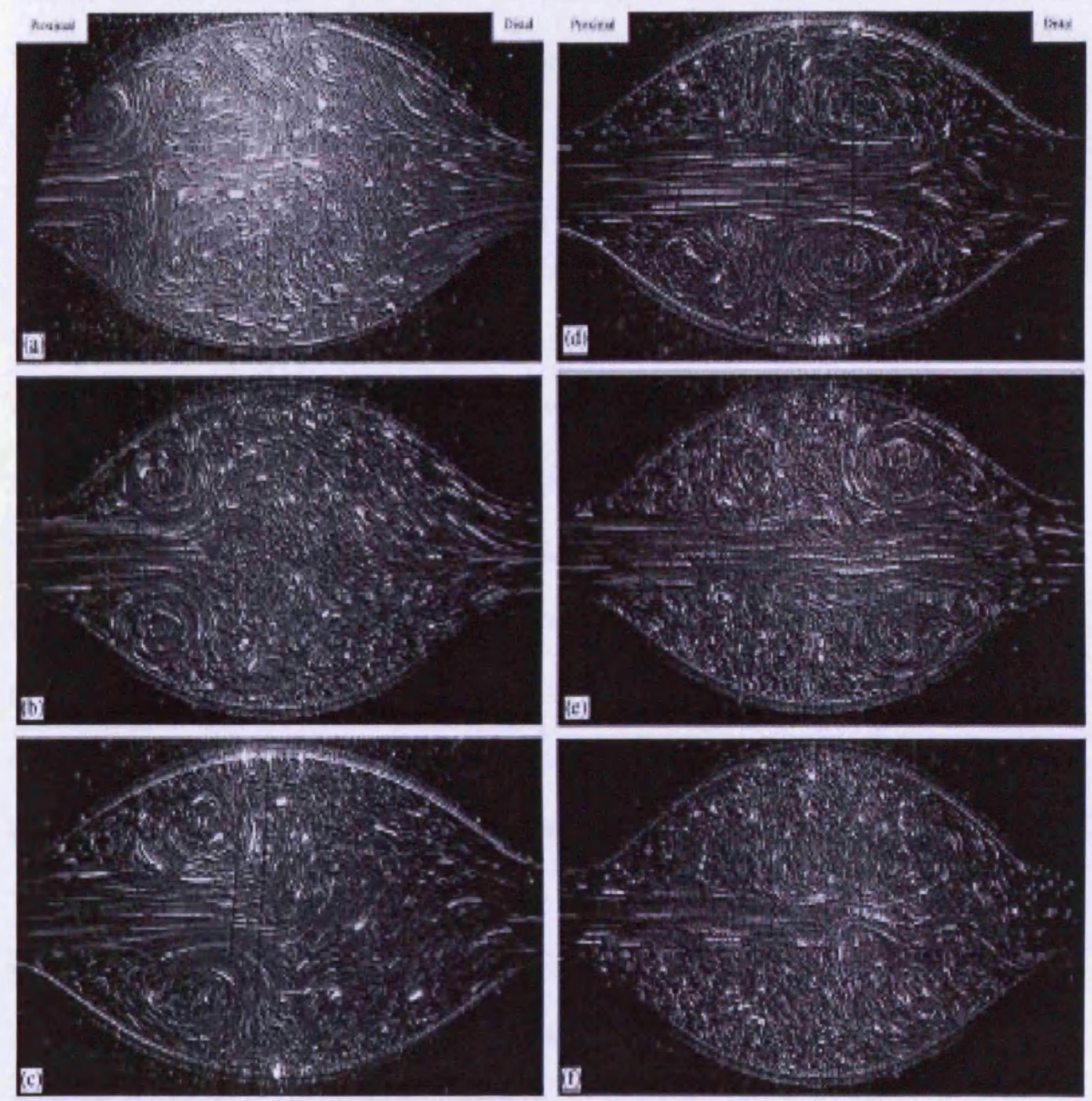

Figure 1.13: Flow development during a flow cycle (Yip and Yu 2001). 
In the small AAA model, input flow with a higher $R e$ number was needed to produce turbulence within the AAA. With $R e=806$, vortices formed inside the bulge half way into the acceleration phase. The vortices were confined to the proximal half of the bulge. Turbulence was detected within the vortex region during the deceleration phase. Unlike the larger model, the vortices did not detach from the wall or move towards the distal end. The study showed that disturbed flow occurred in the AAA models and for laminar input flows, the greater the aneurysm model diameter, the lower the Reynolds number for the onset of turbulence. This agrees with the findings of Peattie and Schrader.

To investigate the effect of forward and reverse input flows on AAA flow patterns, Yip and Yu (Yip and Yu 2002) studied sinusoidal flows through the models. Axial velocities were measured at successive cross sectional planes through the bulge and the cyclic flow development at different phases within the flow cycle was determined. The flow within both the large and small AAA models become increasingly unstable during the forward and reverse acceleration phases and the transition to turbulence occurred shortly after the forward and reverse deceleration phases commenced. The turbulence dissipated during the remainder of each deceleration phase and was destroyed by flow acceleration of the subsequent cycle. In the large AAA model, the flow pattern in the positive half of the flow cycle was similar to that observed in the pulsatile and oscillatory flow regimes: a vortex formed at the proximal end as flow entered the bulge, increased in size and moved towards the distal end during the positive acceleration phase. In the reverse half of the flow cycle, the pattern repeated, but in the opposite direction: a vortex formed at the distal end as flow accelerated in the reverse direction. This vortex grew in size and moved towards the proximal end 
during the negative (reverse) acceleration phase. Turbulence was detected at both ends during the positive and negative deceleration phases. The turbulence at both ends of the model rapidly dissipated as the flow decelerated towards zero. During positive deceleration, the turbulence at the distal end was stronger and more widely spread than at the proximal end. During the negative deceleration phase, the turbulence at the proximal end of the AAA model was stronger and more widely spread than at the distal end. This flow regime was repeated for each flow cycle within the large AAA model. In the small AAA model, three recirculation vortices were formed during the positive acceleration phase as the flow entered the bulge. The recirculation regions increased in size until the end of the acceleration phase. Steep velocity gradients were formed as the flow decelerated and transition to turbulence occurred in the proximal end of the bulge. The turbulence spread along the AAA model wall and its strength decreased as the bulk flow continued to decelerate. During the reverse half of the flow cycle, three recirculation vortices were formed at the distal half of the bulge, and turbulence was detected in this region during the deceleration phase. Again, for each flow cycle the turbulence increased in size and decreased in strength as the bulk flow decelerated, and was destroyed as the flow accelerated.

In vivo, AAAs are seldom symmetrical but more asymmetrical (commonly fusiform/saccular) in nature. Egelhoff et al (Egelhoff, Budwig et al. 1999) compared pulsatile flows in rigid asymmetric and symmetric AAA models of different sizes. Five different size early stage (2-4cm diameter) symmetric AAA models with aspect ratios typical of fusiform aneurysms, and one asymmetric model with one straight and one fusiform shaped side were studied. For the moderate sized symmetric AAA models, vortex formation occurred during late 'systole' (flow acceleration) with 
vortex growth and translation towards the distal end during 'diastole' (flow deceleration). By mid 'diastole', a secondary vortex had formed in the straight inlet tube proximal to the bulge. By late 'diastole', the flow was vortex dominated. The vortex filled the bulge centre and inlet tube proximal to the AAA, and turbulence was observed at these locations. The flow in the large symmetric model was similar to these observations, except that the vortex strength and translation speed were considerably higher. In addition, the vortex was forced out into the exit tube by the accelerating flow of early 'systole'. In the asymmetric AAA model, vortex formation occurred during early systole as for the symmetric situation. However, the strength of the vortex was weaker, there was no transition to turbulence observed and the vortex was not convected into the exit tube.

Yip and Yu (Yip and Yu 2003) also extended their work to investigate sinusoidal flows within idealised, rigid, asymmetric AAA models. They observed that as flow entered the asymmetric AAA model, recirculating vortices were formed either side of the proximal neck, with the vortex on the bulge side larger than on the straight side. As observed in the symmetric model, the vortices grew in size and detached from the walls and moved towards the distal end during the acceleration phase. The vortex on the bulge size was relatively larger at all times. In both the symmetric and asymmetric models, the flow at the distal neck was least stable. In the large symmetric model, turbulence was observed during flow deceleration at the distal end only. In the large asymmetric model, turbulence was observed during flow deceleration at three locations: at the proximal entrance, at the sidewall of the bulge and at the distal neck. The turbulence at the distal neck was double the size of turbulence at the other locations. 
Blood flow patterns are complex and are not easily reproducible because blood vessels are not straight or rigid but are elastic and provide recoil. Therefore, mechanical modelling studies do not strictly reproduce in vivo conditions. However, such studies provide a useful insight into AAA flows which so far have not been successfully studied in vivo.

These modelling studies suggest that disturbed and even turbulent flows are set up within AAAs, altering the normal aortic flow profile. The study by Egelhoff et al suggests that these flow disturbances may be transported into more distal vessels. It may therefore be possible for flow disturbances generated within AAAs to be transported distally into the iliac and femoral arteries.

The main aims of this research project were to determine whether flow disturbances from an AAA can be detected in the common femoral artery at the top of the leg using Doppler ultrasound, and whether detection of the flow disturbances could be used as an alternative method for AAA detection.

\subsubsection{Physiological Effects of AAA Flow Patterns}

The mechanical modelling studies also indicated that the type of flow within an AAA model is dependant on the physical aspects of the AAA. In all models, the bulk flow acceleration reduced flow instabilities inside the AAA, but deceleration facilitated instability growth. The flows observed in the models have physiological implications and may contribute to the development and rupture of AAAs. The aortic wall is subjected to cyclic and turbulent, normal and shear stresses due to the cyclic and 
turbulent flow patterns set up within the aneurysm. During acceleration, the flow is stable and the walls experience laminar shear stresses only. During deceleration, turbulent flows subject the walls to high turbulent shear stresses. For sinusoidal flows Yip and Yu (Yip and Yu 2002; Yip and Yu 2003) estimated the wall shear stress from the instantaneous velocity profiles at different phases in the flow cycles. The peak stress was observed at the distal end of the aneurysm models during the deceleration phase. This is because the onset of turbulence at the distal neck during the deceleration phase subjected the wall at this location to high turbulent shear stresses. This correlates with clinical observation that most aneurysms rupture at the distal end.

It is understood that cells sense and respond to their biomechanical environment. Endothelial cells respond to the shearing forces from flowing blood and mechanical stretch from arterial pulsation. Therefore, biomechanics plays a key role in the development, enlargement and rupture of aortic aneurysms (Steinman, Vorp et al. 2003). Turbulent shear stresses are much more damaging to endothelial cells than laminar shear stresses of the same magnitude (Yip and Yu 2003). Turbulent fluid stress is known to induce and promote vascular endothelial cell turnover and may cause blood cell and platelet damage. Therefore, the turbulence observed in the AAA models may explain the observed build up of intra-luminal thrombus (ILT) on the AAA wall in vivo. The turbulent flows in large AAAs are less damaging to free moving blood cells and platelets but still cause endothelial layer damage (Yip and Yu 2001).

At present, elective repair is based on the maximum diameter of the aneurysm. However, AAAs with the same diameter may have different wall thickness, shape and 
mechanical properties which effect the growth and likelihood of rupture. From a biomechanical point of view, the critical state of an AAA is when the mechanical stress within the aneurysmal wall exceeds the tensile strength of the tissue (Vorp, Raghavan et al. 1998). It is suggested that evaluation of wall stress distribution may provide a predictor for rupture risk. Therefore, in addition to detecting AAA from disturbances in distal flow, the disturbance patterns may possibly also provide an indication of rupture risk.

\subsubsection{Numerical Fluid Dynamics Modelling}

Sophisticated image processing and computational fluid dynamics (CFD) modelling techniques can provide reconstruction of the time varying, three dimensional blood flow patterns within realistic arterial models (Steinman 2002). The first image based CFD studies of arterial geometries were based on angiographic x-ray images. More recently, CT angiography and MRI have been used to reconstruct more anatomically realistic arterial geometries. MRI has become increasingly popular for imaging blood vessels because it does not require the administration of intravenous contrast agents. This is because the contrast is achieved by exploiting the differences in magnetic spin relaxation properties between tissue types. The properties of blood itself provides contrast with the surrounding tissue. MRI is also a powerful technique for imaging in vivo blood velocity using a method called phase-contrast MRI (PC-MRI). In this technique, the blood flow velocity is encoded into the phase of the complex MRI signal. Time-resolved 2-D or 3-D images of one or more velocity components can be displayed. Spatially resolved velocity maps can be produced but at the expense of the temporal resolution. PC-MRI can measure time-varying velocities under steady flow 
conditions, however the velocity images can become distorted by a number of atrifacts in the presence of complex flow patterns (Steinman 2002). It has therefore not been possible to use this technique to examine the flow disturbances within AAAs.

Ultrasound imaging of vascular anatomy, although clinically widely used, does not play a significant role in CFD analysis. This is because 2-D ultrasound images are usually acquired manually without reference to a fixed co-ordinate system, making it difficult to accurately reconstruct 3-D views. 3-D ultrasound systems that sweep the beam either mechanically or electronically to acquire a series of orthogonal 2-D images through a 3-D volume are available, however, the overall image quality is not sufficient for reliable CFD calculations. Therefore, MRI and CT angiography are the preferred techniques for producing anatomically realistic arterial models. Doppler ultrasound is useful in CFD to provide real-time measurements of blood flow velocity, vessel diameter and flow rates. To date, fluid dynamics modelling has mainly been used to predict AAA wall stress rather than to investigate flow patterns.

Vorp et al (Vorp, Raghavan et al. 1998) used finite element analysis to determine the effects of AAA shape and diameter on the wall stress distribution in AAAs. The results indicated that the asymmetry of an AAA in addition to aneurysm diameter is important for determining mechanical wall stress. Aneurysms with the same diameter do not necessarily have the same likelihood of rupture. This may be important to consider in the clinical management of AAAs. 
Hua et al (Hua and Mower 2001) applied linear finite element analysis to mathematical AAA models to investigate the relationship between peak wall stresses and AAA volume, maximum diameter (for symmetrical AAAs), maximum radius (for asymmetrical AAAs), aspect ratio and maximum wall distension. The results showed an increase in peak wall stress as the AAA became increasingly asymmetrical. This is in agreement with the analysis by Vorp.

Fillinger et al (Fillinger, Raghavan et al. 2002) used finite element analysis on AAA models reconstructed using CT scan data to calculate AAA wall stresses in vivo for ruptured and electively repaired AAAs. The location of the maximum stress was on the postero-lateral wall where clinically most ruptures occur, not at the site of maximum diameter. Again, it was concluded that the AAA geometry appears to assess rupture risk more accurately than AAA diameter.

\subsubsection{Effect of Intra-luminal Thrombus}

The role of intra-luminal thrombus (ILT) within aneurysms is controversial. Embolisation of thrombus from within an AAA is common and can cause ischemia and necrosis of tissues. Many factors contribute to the stress within an AAA wall including surface curvature, wall tissue characteristics and the presence of ILT (Di Martino and Vorp 2003). A number of mechanical, numerical and in vivo experiments have been performed to evaluate the effect of intra-luminal thrombus on the pressure exerted on the aneurysm wall and the stress distribution. Schurink et al (Schurink, van Baalen et al. 2000) performed in vivo pressure measurements within aneurysmal thrombus during aneurysmectomy and showed the difference between the mean 
arterial pressure and the mean pressure within the thrombus were not related to the thickness of the thrombus at the level of the pressure measurement.

It has been demonstrated by other authors that ILT can significantly alter the wall stress distribution in an AAA. Martino et al (Di Martino and Vorp 2003) investigated the effect of ILT thickness on the wall stress distribution using finite element analysis. The stress distribution was computed for 3-D, asymmetric AAA models with ILT of different constitutive parameters and uniform applied systolic pressure $(120 \mathrm{mmHg})$. The presence of ILT, regardless of its mechanical properties redistributed the wall stress. Altering the mechanical properties of the ILT had a moderate effect on the distribution and peak stress. In all cases the maximum stress distribution occurred on the posterior wall. The load was sustained almost completely by the aortic wall, rather than the ILT. There was no significant difference between the stress distribution for homogeneous and inhomogeneous ILT.

These ILT studies indicate that ILT may significantly alter the stress distribution at the aortic wall. These results may support the clinical observations which indicate that the presence of ILT is associated with increased rupture risk. The shape and size of the aneurysm has also been shown to affect the flow patterns and wall stress. Because blood flow patterns are dependant on the artery size, shape and elasticity, analysis of the distal Doppler waveform could possibly provide information on the size, shape and possibly even the extent of thrombus within an AAA. 


\subsubsection{Detection of AAA Flow Disturbances In Vivo}

The primary aims of this research project were to determine whether AAA causes disturbance to the normal aortic flow, whether the disturbances are propagated distally and if they can be detected using Doppler ultrasound. The distal distance at which it may be possible to detect the any flow disturbances set up within an AAA will depend on the strength of the disturbance and the impedance to flow in the iliac and femoral vessels. The most proximal vessel distal to the aorta which can be most readily assessed using Doppler ultrasound is the common femoral artery (CFA).

This work came about because Dr Neil Pugh who has over 20 years vascular experience in vascular ultrasound noted during routine clinical assessments for peripheral vascular disease that when certain changes were observed in the CFA waveform an AAA was often present on examination of the aorta. An example of the modifications to the normal CFA waveform measured using colour flow duplex imaging from a patient with an AAA are shown in Figure 1.14. The CFA waveform in the presence of an AAA shows high levels of spectral broadening, typical of disturbed or turbulent flow in the deceleration phase of the systolic peak. These changes seem to be consistent with the intermittent turbulent bursts observed in the flow within an AAA model during the deceleration phase of the flow cycle in the study by Yip and $\mathrm{Yu}$ (2003) shown in Figure 1.15. This suggests that AAA may alter the distal flow pattern. Therefore it may be feasible to detect the presence of AAA by analysis of the CFA Doppler waveform. 


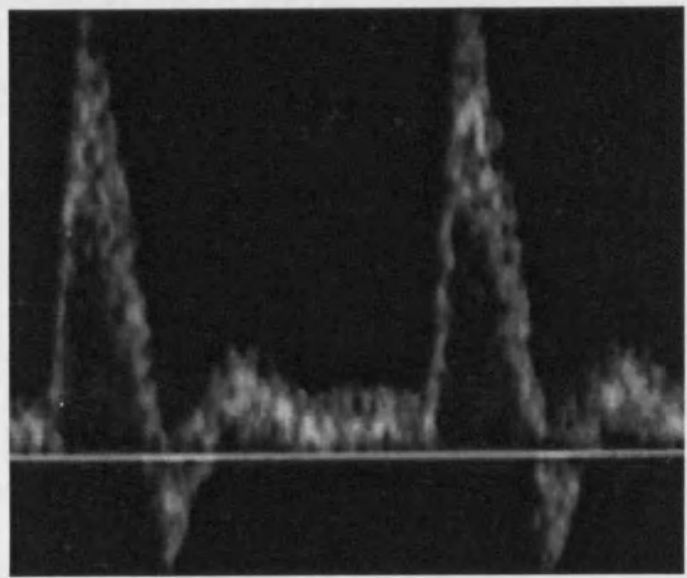

a)

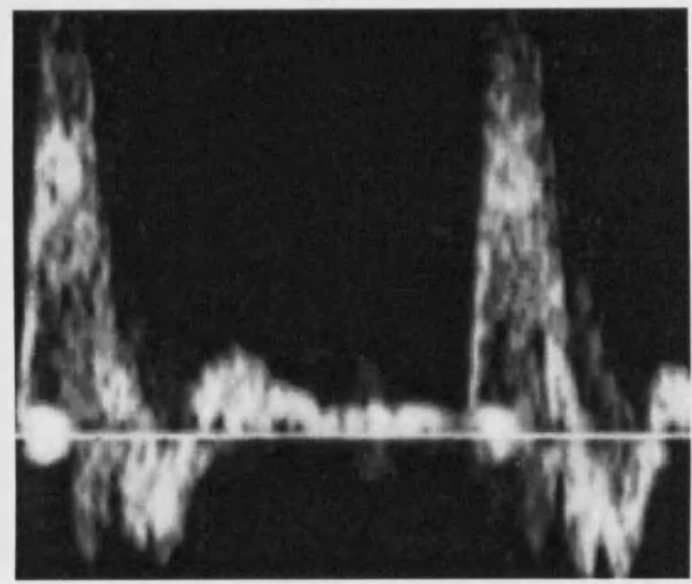

b)

Figure 1.14: Modification of CFA waveform in the presence of AAA.a) CFA waveform from patient with normal calibre aorta. b) CFA waveform from patient with $4.6 \mathrm{~cm}$ AAA.

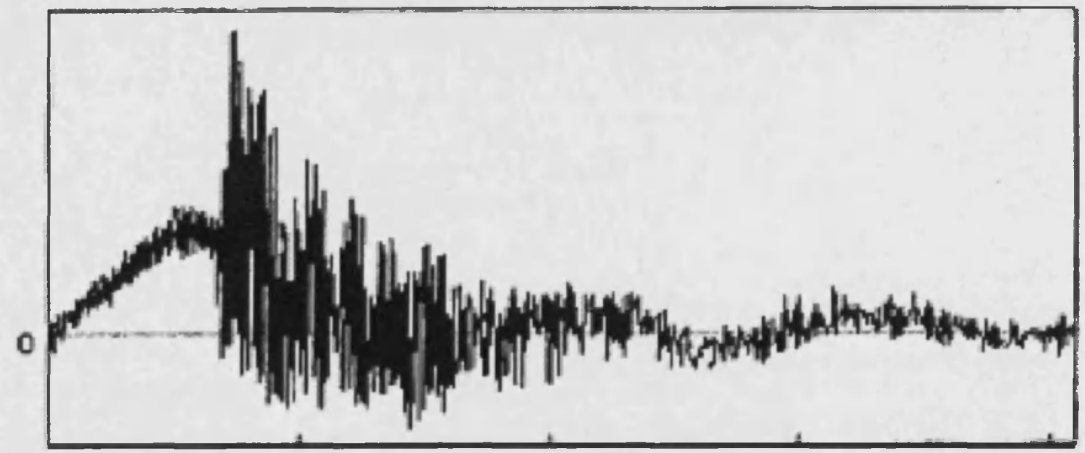

Figure 1.15: Phase variation of streamwise velocity measured where turbulent bursts appeared during deceleration phase in AAA model (Yip and Yu 2003) 
The results of an early study by Humphries et al (Humphries, Hames et al. 1982) which aimed to detect arterial dilatation (arteria magna) from continuous wave Doppler waveforms of the lower limb palpation sites supports these observations. The study was based on Doppler shift signals obtained simultaneously from the common femoral, popliteal, posterior tibial and dorsalis pedis arteries in 54 patients with angiographic, B-mode or surgical confirmation of arterial dilatation. The Doppler shift signals from each artery were displayed in the form of a spectrogram and the maximum frequency envelope (MFE) was calculated. The results showed that indices such as PI or the Laplace damping factor $(\delta)$ calculated from the MFE were unable to adequately indicate arterial dilatation. There were however characteristic and distinctive Doppler shift waveform patterns produced by general dilatation and AAA. In patients with $\mathrm{AAA}$ and no occlusive arterial disease, the waveform at the common femoral artery had a characteristic flattening or 'knee' on the first forward flow component as illustrated in Figure 1.16, which manifested as a dissociation of the first and second (forward and reverse) components at the posterior tibial artery.

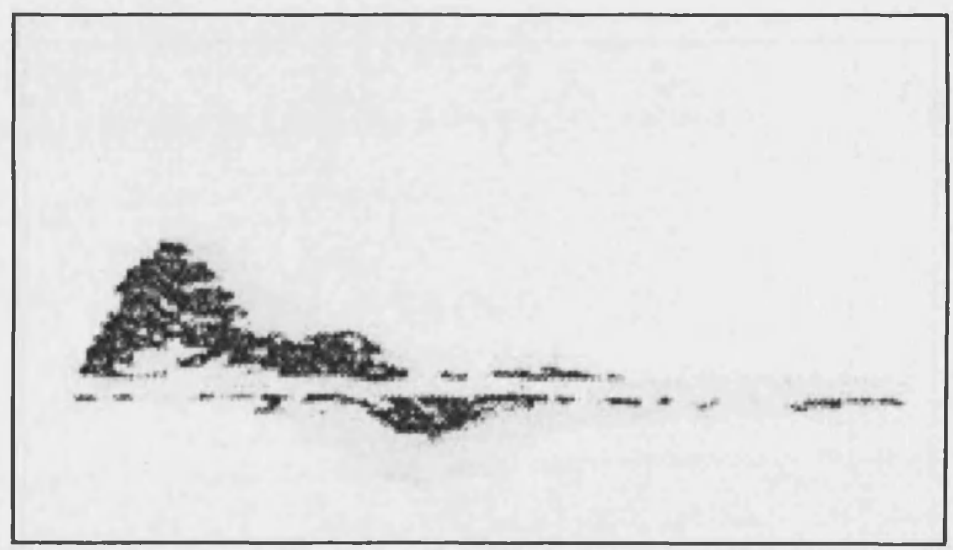

Figure 1.16: Characteristic flattening or 'knee' on the first forward flow component of CFA waveform in patient with AAA (Humphries, Hames et al. 1982). 
The CFA waveform from a patient with a normal calibre aorta and SFA occlusion also showed a flattening but this was at a higher level on the downward slope than was seen with AAA or dilatation as illustrated in Figure 1.17. The CFA waveforms from patients with dilatation and or tortuosity also had a characteristic signature of a skewed first component (systolic peak) followed by a reverse flow component extending over the remainder of the cardiac cycle as illustrated in Figure 1.18.

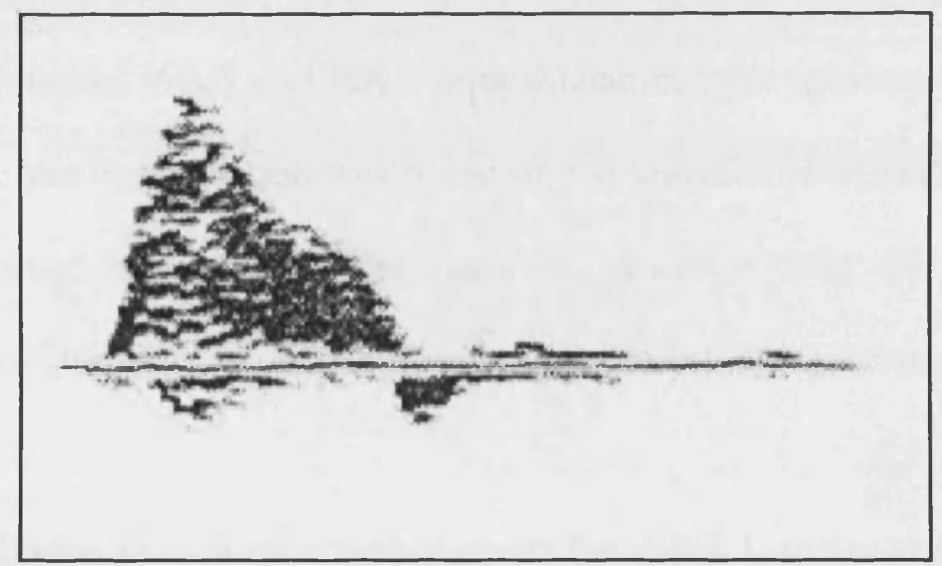

Figure 1.17: Flattening of first forward flow component of CFA waveform with SFA occlusion (Humphries, Hames et al. 1982).

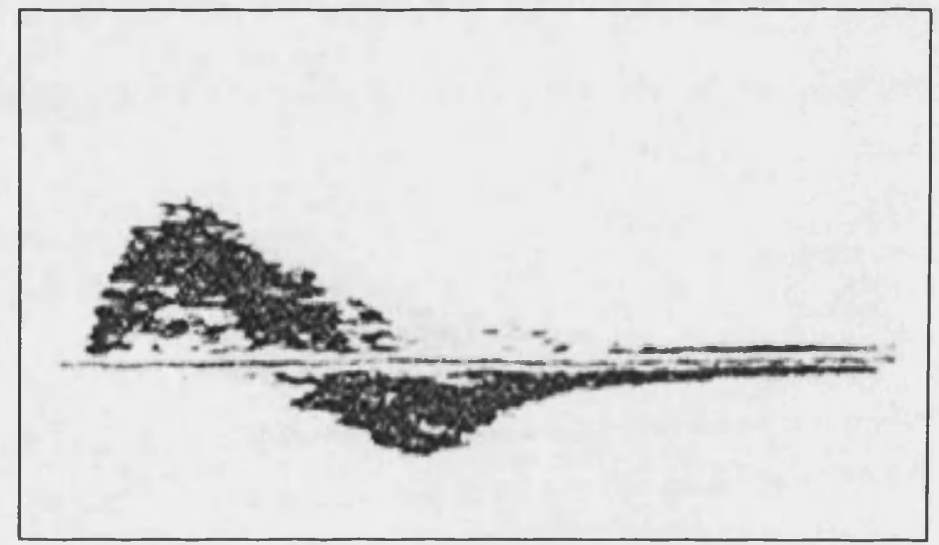

Figure 1.18: Reverse component of CFA waveform extending over the rest of the cardiac cycle with proximal dilatation and tortuosity (Humphries, Hames et al. 1982). 
A qualitative assessment of dilatation was made based on the recognition of 3 waveform signatures: 1) waveforms with an flattening or 'knee' in the first flow component, 2) waveforms with a skewed first component and a reverse component extending over the remainder of the cardiac cycle, and 3) waveforms with a reverse frequency peak within the time period of the first forward component. When these classification criteria were applied by visual inspection of the CFA waveforms, success rates for predicting generalised dilatation, AAA and AAA with dilatation were $76 \%, 41 \%$ and $36 \%$ respectively. In the case of AAA with associated occlusive arterial disease the sensitivity was only $7 \%$. The sensitivities in the detection of generalised dilatation, $\mathrm{AAA}$ and $\mathrm{AAA}$ with dilatation were increased to $85 \%, 56 \%$ and $50 \%$ when the classification was based on the waveforms collected from all the measurement sites: common femoral, popliteal, posterior tibial and dorsalis pedis arteries. The sensitivity to AAA with associated occlusive disease was unchanged.

This study indicates that there are changes to the distal Doppler waveforms in the presence of arterial dilatation and/or AAA. Pattern recognition of the Doppler waveforms was successful in diagnosing general arterial dilatation but was less sensitive to the diagnosis of AAA, and was particularly poor in cases with associated occlusive disease. 


\subsection{Discussion and Conclusions}

Studies have shown that screening for Abdominal Aortic Aneurysm (AAA) using conventional ultrasound techniques is cost effective. While screening is desirable, conventional ultrasound scanning is generally hospital based and requires a specialist operator to perform the investigation and interpret the results. Theoretically the presence of an AAA should change the aortic flow pattern and these changes should influence the characteristics of Doppler waveform in distal arteries. The AAA modelling studies indicate that disturbed and possibly turbulent flows occur within AAAs and that these disturbances may be transported distally. In theory, it may be possible to detect the flow disturbances in the large arteries of the lower limb using Doppler ultrasound. The observation of spectral broadening in the deceleration phase of the flow cycle in the CFA waveform during routine clinical examination seems to be consistent with the disturbed flows observed in the modelling studies. This is also supported by the observations by Humphries who demonstrated that characteristic and distinctive Doppler waveform signatures at the lower limb palpation sites were produced by general dilatation and AAA. These studies and observations suggest it may be feasible to detect the presence of AAA by analysis of the CFA waveform.

The studies by Peattie et al and Yip and Yu indicate that the flows set up within an AAA model are dependant upon the AAA size and shape. Yip and Yu also indicated that these flow patterns can significantly affect wall stress and its distribution and may contribute to the development and rupture of AAAs. Turbulent fluid stress may promote the build up of ILT on the aneurysm wall. Studies also show that ILT possibly redistributes the stress at the aortic wall. At present elective AAA repair is 
based on the maximum diameter of the aneurysm. However, AAAs with the same diameter may have different mechanical properties which could effect the growth and likelihood of rupture. Because the flow patterns in the AAA models were dependant on the physical aspects of the aneurysm models, analysis of the CFA waveforms could also possibly provide data on the size of the aneurysm, and even the presence of ILT. This data is useful to clinicians due to the associated implications for management and treatment options. Because turbulent flows appear to increase wall stress, if turbulent flows can be detected in the distal waveform in this research, this may be an aid for the prediction of rupture risk in future studies.

The main aim of this research project was to develop an alternative method for AAA detection by analysis of the CFA waveform. This involved a formal investigation to determine if and how AAA changes the characteristic CFA waveform, followed by the development of an objective, automated analysis technique to detect the changes and determine whether or not an AAA is present. The second aim was to determine whether the technique could also be used to provide information to the clinician on aspects such as the size and presence of thrombus in the AAA.

The interpretation of Doppler waveforms requires a high level of skill and training but still remains subjective. The analysis is complicated further by changes to the waveform due to occlusive arterial disease. In Humphries early study, prediction of AAA by classification of the CFA waveform was relatively low $(<50 \%)$ and very poor for AAA with associated occlusive arterial disease. The waveform changes were completely removed by the presence of occlusive arterial disease proximal to the measurement site. It was therefore necessary to investigate the combined effects of 
occlusive arterial disease and AAA on the distal waveform. In some cases the changes to the distal waveforms may be subtle and may not be obvious on visual examination of the waveforms. More complex and objective analysis may be necessary to detect characteristic alterations. It was decided that it may be viable to use MATLAB software based analysis of the CFA waveforms to determine objectively whether or not an AAA is present. An overview of the general methods and equipment used to conduct the research study is given in Chapter 2.

CFA Doppler waveforms are routinely measured by GPs and outpatient nurses using a simple hand held 'Pocket Doppler system'. If it proves possible to objectively detect AAA from CFA the waveform. It may be possible in future work to develop a handheld screening tool for AAA which could provide a quick and more cost effective method for screening in GP surgeries, outpatient clinics and home visits to indicate the need for a full ultrasound examination. 


\section{Chapter 2: Equipment and General Methods}

This chapter is an overview of the general methods and equipment used to conduct the research study. The data was collected using two main ultrasound modalities: ultrasound imaging to assess the arteries for aneurysmal and atherosclerotic disease and Doppler ultrasound to assess and measure the blood flow. The first section of this chapter gives an overview of the study design. The second section describes the arterial imaging equipment and methods. The third section describes the equipment and methods used for Doppler waveform measurement and the fourth section describes the general signal processing methods. A summary of the collected data is given in the fifth section.

\section{$\underline{\text { 2.1 Study Design }}$}

Doppler waveforms were measured from the CFA in 70 patients with AAA ('cases') and 70 patients without AAA ('normals'). For each patient, a B-mode ultrasound scan of the aorta was performed to confirm and measure the AAA for the 'cases' or rule out the presence of AAA for the 'normals'. Because the Doppler waveform in an artery is changed in the presence of atherosclerotic or further aneurysmal disease, a bilateral scan of the iliac and lower limb arteries was performed on all subjects to rule out or determine the extent of atherosclerotic or further aneurysmal disease. Both the scans were performed following the standard clinical protocols described in sections

2.2.2 and 2.2.3. All subjects were over 50 years of age. This age criterion was set to obtain a similar age range in the aneurysm and non-aneurysm groups. This was necessary because the elasticity of arteries within the body naturally decreases with 
age, causing minor alterations to the Doppler waveforms (Nichols and O'Rourke 2005).

The data from the AAA and no AAA patient groups were divided into two main categories: the first category comprised the patients with no significant atherosclerotic disease of the iliac or femoro-poplitael arteries and the second category comprised the patients with 1 or more significant stenotic or occlusive atherosclerotic lesions within the iliac, femoral or popliteal arteries. The data from the patients in these two main categories were dealt with separately, naturally dividing the study into 2 main sections. The research process for each section involved 2 main stages. The first stage was the identification of features which may be characteristic of AAA by visual inspection of the CFA waveforms in each group. In the second stage, the waveforms were processed using MATLAB software to analyse the waveforms and extract a set of meaningful parameters to best represent the visual features. A computer program was then established using MATLAB software to perform the analysis objectively and to classify the waveforms in terms of AAA according to the parameters representing the characteristic features. The ability of the classification technique to correctly diagnose AAA from the CFA waveform was determined. The first and second research stages for the patients with no significant iliac or femoro-poplitael arterial disease are described in Chapters 3 and 4 of this thesis respectively. The first and second research stages for the patients with significant iliac or femoro-poplitael arterial disease are described in Chapters 5 and 6 of this thesis. In the final stage of the research discussed in chapter 7 , the relationship between the characteristic waveform features and the physical characteristics of AAAs were investigated. A discussion of the main results and conclusions is given in Chapter 8 . 


\subsection{Arterial Imaging Methods}

The B-mode ultrasound scans of the aorta to confirm or exclude AAA and the bilateral Doppler ultrasound scans of the lower limb arteries to rule out or determine the extent of atherosclerotic or further aneurysmal disease were performed on all patients using a Toshiba Xario ultrasound system. An overview of this system and the protocols followed for each part of the scan are detailed below.

\section{$\underline{\text { 2.2.1 Toshiba Xario System }}$}

The Toshiba Xario system (Toshiba Medical Systems, Crawley, UK) is one of two Toshiba systems routinely used for clinical assessment of vascular disease in the Doppler Ultrasound Department at UHW. It is a sophisticated colour flow duplex system, optimised for the assessment of peripheral vascular disease and has a range of transducers available for different vascular imaging applications. For this study the lower limb arteries were assessed using the PLT-604AT linear probe employing tissue harmonic imaging at $6.6 \mathrm{MHz}$ and pulsed wave Doppler at $4 \mathrm{MHz}$ fundamental frequency. The aorta and iliac arteries were assessed using the PVT-375BT curvilinear probe employing tissue harmonic imaging at $4.0 \mathrm{MHz}$ and pulsed wave Doppler at $1.8 \mathrm{MHz}$ fundamental frequency.

\subsubsection{AAA Scan Protocol}

In scanning the aorta, the patient lay supine on the scanning couch with the lower abdomen exposed. The scan was performed using the curvilinear probe and the 
'Abdominal Vascular' pre-set. The distal aorta was imaged by placing the ultrasound transducer on the lower abdomen with contact gel. The aorta was imaged in longitudinal and transverse planes and the maximum diameter of the aorta was measured in each plane. If the diameter of the distal aorta exceeded $3 \mathrm{~cm}$ it was classed as aneurysmal. If ILT was present within an AAA the diameter of the residual lumen was measured in longitudinal and transverse planes. All images were stored onto the scanner memory. Examples of the transverse and longitudinal measurements of an AAA and residual lumen diameter for an AAA with ILT are given in Figure 2.1.

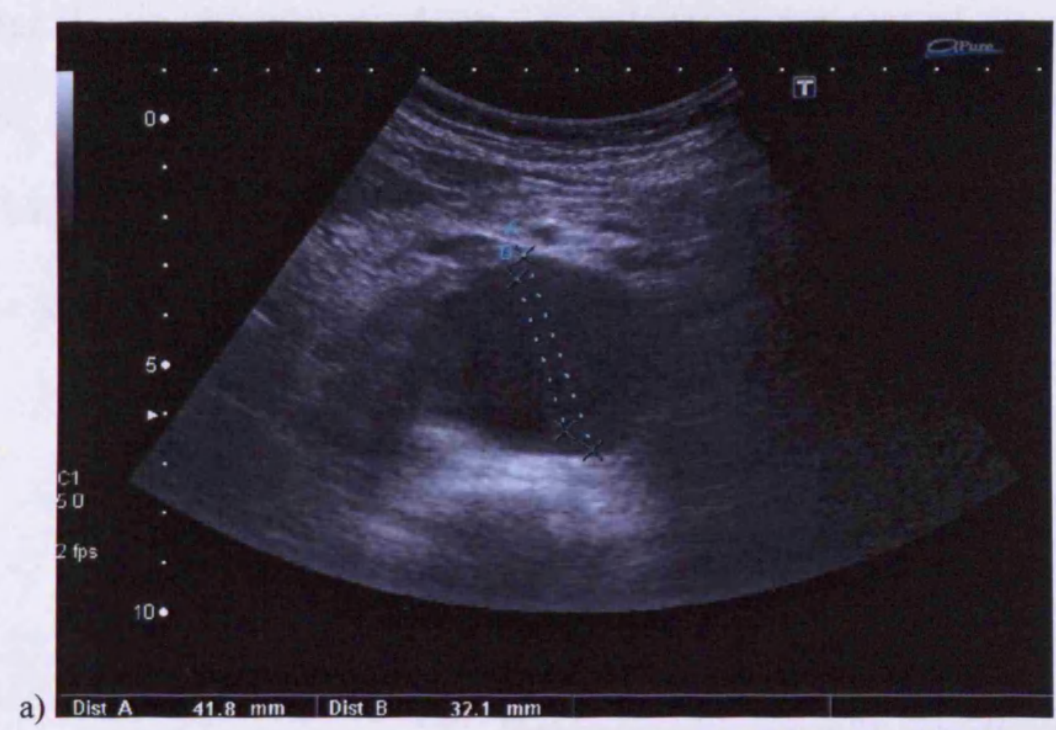

a)

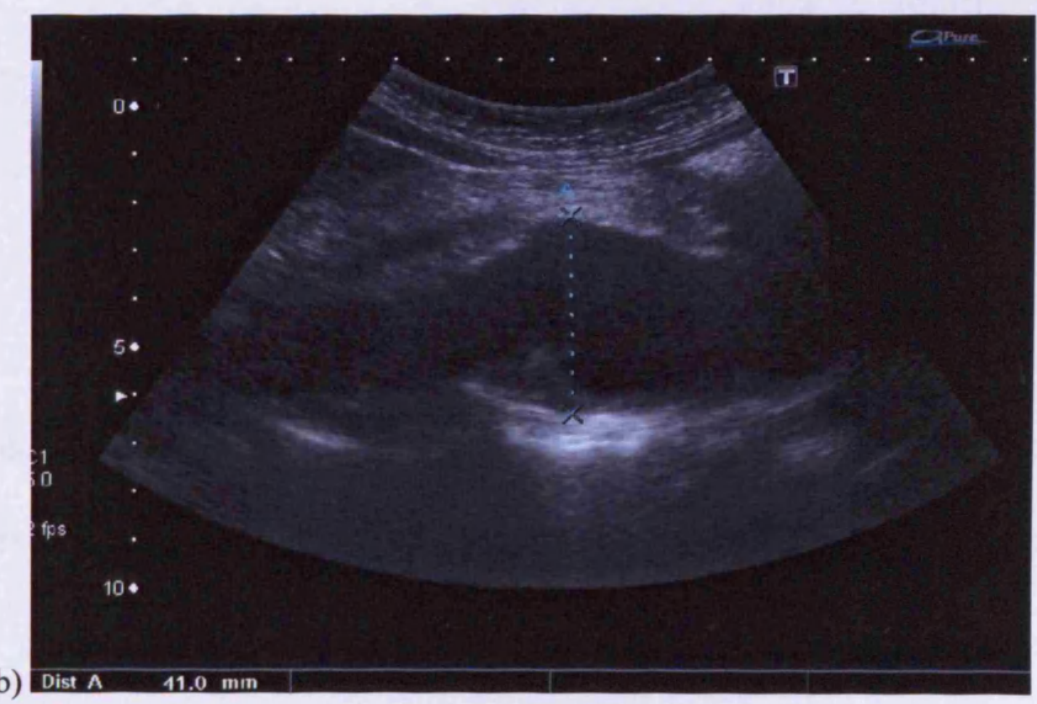

Figure 2.1: Maximum AAA and residual lumen diameters in a) transverse plane and b) longitudinal plane 


\subsubsection{Lower Limb Arterial Scan Protocol}

In scanning the lower limb arteries, the patient lay supine on the scanning couch with the legs and lower abdomen exposed. The iliac arteries were imaged using the curvilinear probe and Abdominal Vascular pre-set. The blood flow in the common iliac arteries (CIAs) and external iliac arteries (EIAs) were assessed bilaterally using colour flow Doppler and triplex functions. The CFAs, SFAs and popliteal arteries were assessed bilaterally using the linear probe and lower limb arterial scan pre-set. The CFA, SFA and popliteal arteries were imaged in longitudinal plane using Bmode, colour flow and triplex to determine patency and extent of disease. Disease severity was assessed using triplex mode to the measure ratio of the peak systolic velocity (PSV) from the Doppler waveform just proximal to and through the stenosis. The disease severity was classified using the following standard criteria given in Table 2.1.

\begin{tabular}{|c|c|}
\hline PSV ratio & \% Stenosis \\
\hline$<2$ & Not haemodynamically significant \\
\hline 2 & $50 \%$ (moderate) \\
\hline$>3$ & $>70 \%$ (tight) \\
\hline No colour flow & Complete Occlusion \\
\hline
\end{tabular}

Table 2.1: PSV ratios for stenosis classification

A complete occlusion was confirmed by reducing the colour scale and/or using Power Doppler. The presence of collateral vessels and the level of reconstitution were recorded. 


\subsection{Waveform Measurement Methods}

\subsubsection{Waveform Measurement Equipment}

The Toshiba Xario system permits pulsed wave Doppler measurements in duplex or triplex mode. The Doppler shift data is displayed as an on-screen spectrogram and is also output audibly via an in-built speaker. Because the system was designed for routine clinical assessment of blood flow, it did not permit the Doppler shift signals to be output in a format which could be analysed by an external computer. Therefore a software package called DopStudio (Skidmore Medical, Bristol, UK) was used to record the Doppler shift signals from the Toshiba Xario system onto a laptop PC. DopStudio samples an audio Doppler shift signal input from an external Doppler system via a USB sound card. The incoming Doppler shift signal is sampled at $48 \mathrm{kHz}$ and the sampled frequency data is displayed in the form of a spectrogram. The DopStudio software was modified by the manufacturer for this research project to convert the Doppler frequency shift data into CSV file format to facilitate analysis in MATLAB software. In order to output the Toshiba Xario audio Doppler signal a Toshiba engineer activated the headphone sockets on the system and attached wires for connection into the laptop soundcard. The composite waveform measurement system is referred to in this thesis as the Xario-DopStudio system.

\subsubsection{Measurement Protocol}

The CFA Doppler waveforms recorded using the Xario-DopStudio system were obtained using the Xario triplex function to visualise the CFA to enable the Doppler 
gate at to be placed at mid-CFA level to avoid flow disturbances which can occur around the bifurcation. The gate was positioned at the vessel centre and the gate width was increased to encompass $\sim 75 \%$ of the patent lumen. The Doppler angle cursor was aligned with the flow and the probe angle was adjusted if necessary to maintain a Doppler angle of $<60^{\circ}$. The 'update' function was used to increase the Doppler PRF scale to remove signal aliasing in the on-screen DopStudio spectrogram The DopStudio spectrogram timescale was set to $5 \mathrm{~s}$. The Doppler gains on the Toshiba Xario system and the DopStudio system were altered to minimise background noise and optimise the waveform signal observed in the DopStudio spectrogram. The DopStudio spectrograms were saved in CSV file format in the laptop computer memory. The on-screen displays of the spectrograms on DopStudio and the Toshiba Xario systems were also saved in the respective system memories.

\section{$\underline{\text { 2.3.3 Comparison of Toshiba Xario and DopStudio Waveforms }}$}

A short study was performed to examine the quality of the waveforms recorded using the Xario-DopStudio system. CFA waveforms from 5 AAA and 5 'normal' patients measured using the Toshiba Xario system and the Xario-DopStudio system were visually compared.

The Toshiba Xario waveforms were reproduced well by the DopStudio system. All the main features were preserved. The only main difference between the waveforms were that the Xario-Dopstudio system signals were more prone to background noise but the noise was not excessive. The Xario-Dopstudio system had a higher level high pass 'wall thump' filter resulting in a signal-free band either side of the zero axis. 
However, all other waveform features were well preserved by the system. It was decided that the Xario-Dopstudio waveforms were of sufficient quality for the data collection. An example of a CFA waveform measured using the Toshiba Xario system and the corresponding waveform displayed by DopStudio are shown in Figure 2.2.

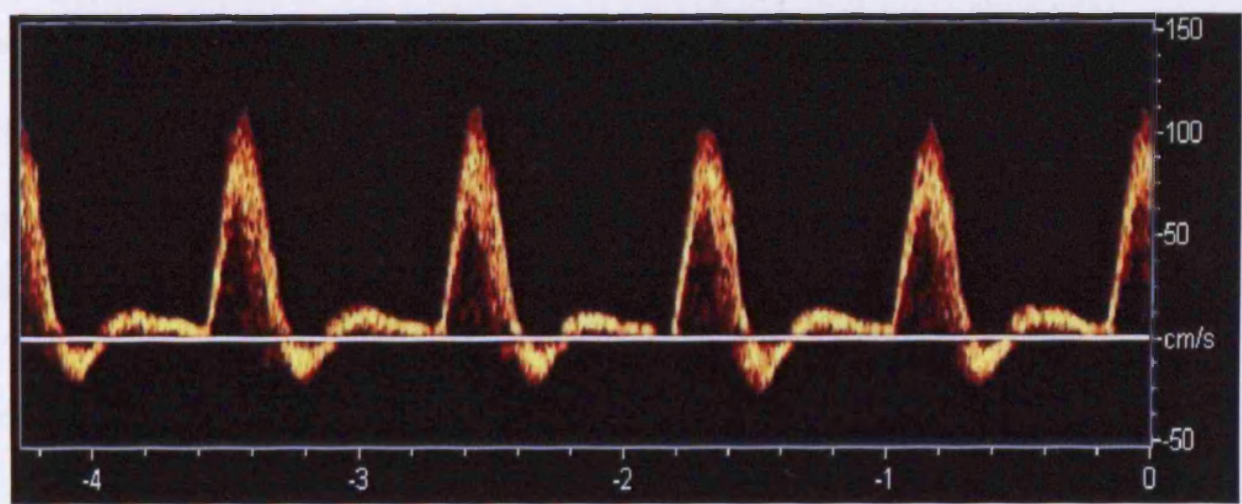

a)

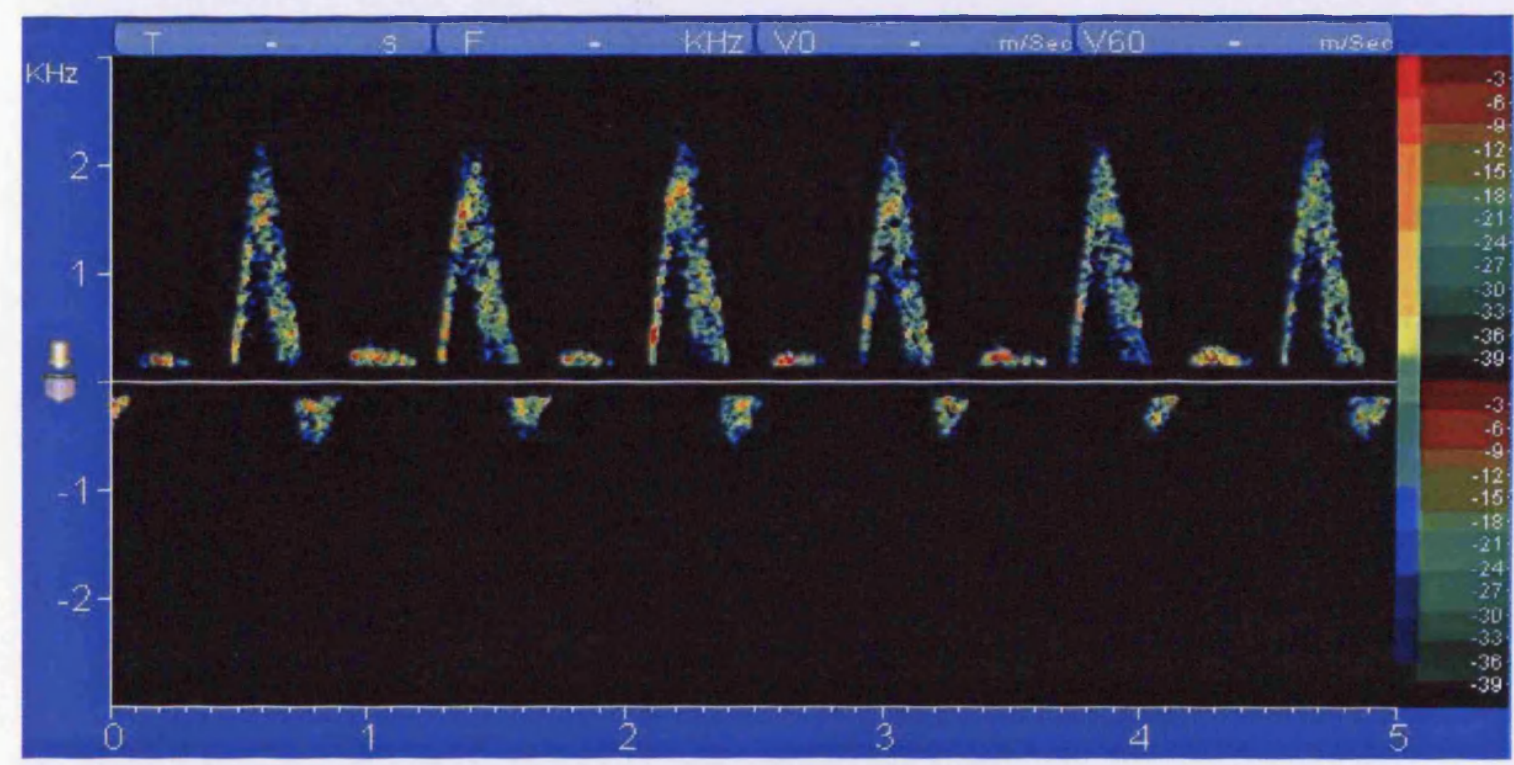

b)

Figure 2.2: a) CFA Waveform measured with the Toshiba Xario System, b) corresponding waveform displayed by the DopStudio system. 


\subsection{Input Data}

The CFA Doppler shift signals recorded using the Xario-DopStudio system and saved in CSV file format were transferred to a desktop PC for analysis. All signal processing for this study was performed using MATLAB software (The Math Works Inc, Natick, MA, USA). The CSV files were read into MATLAB and stored as a matrix. The DopStudio data was output as a 256 by 500 matrix, with 500 columns representing the time base and 256 rows containing the Doppler shift frequency amplitudes of the spectrogram. The magnitude (power) of the Doppler shift signal is stored as an integer in each matrix element. The CFA spectrograms were reproduced using the 'imagesc' plotting function in the MATLAB software. Figure 2.3 shows the reproduction of the CFA waveform shown in Figure 2.2 using the MATLAB 'imagesc' function.

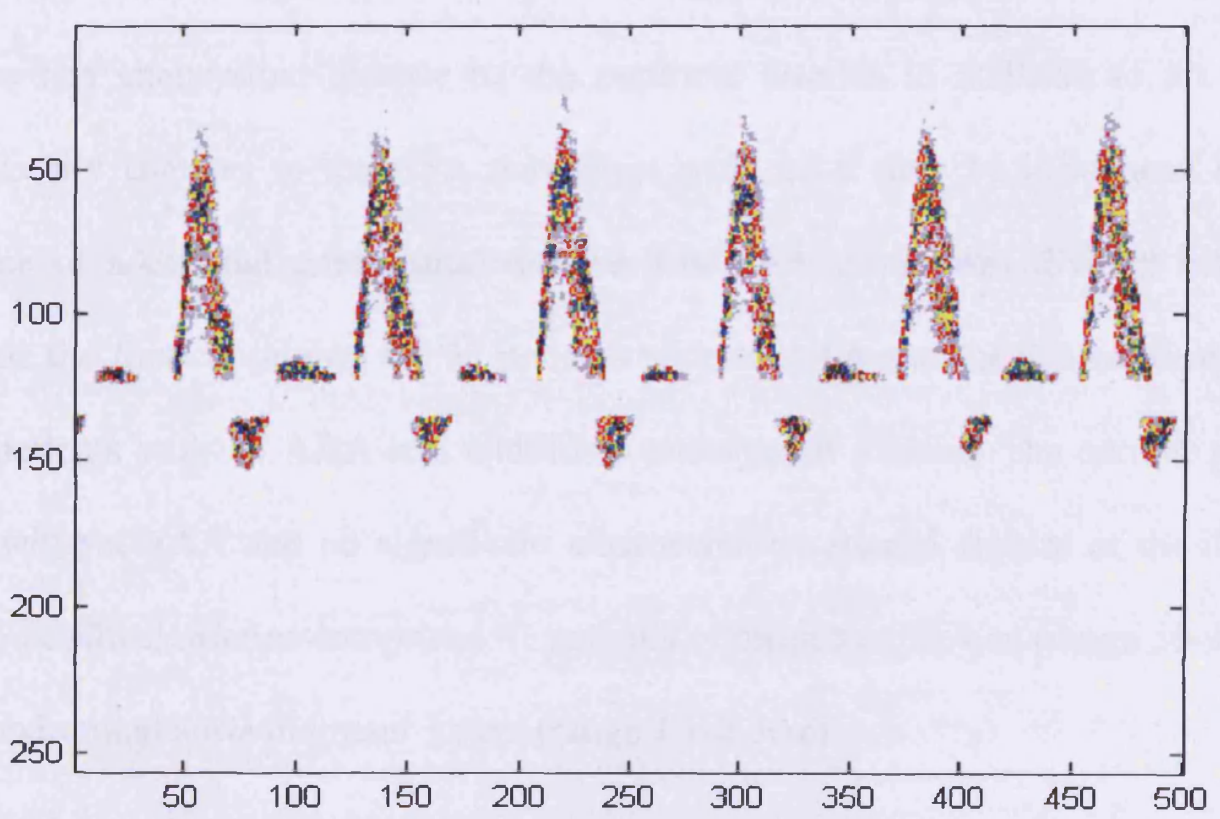

Figure 2.3: Xario-DopStudio CFA waveform reproduced in MATLAB 


\subsection{Data Summary}

The 70 patients with AAA and 70 patients without AAA were divided into 4 main groups. The first group contained patients with AAA and no significant iliac or femoro-popliteal atherosclerotic disease. The second group contained patients without AAA and no significant iliac or femoro-popliteal atherosclerotic disease. The third group contained patients with AAA and with significant atherosclerotic disease of the iliac and/or femoro-popliteal arteries. The fourth group contained patients without AAA but with significant atherosclerotic disease of the iliac and/or femoro-popliteal arteries. A summary of the patient data in each group is given in Table 2.2. More detailed summaries of the patient data for each group are given in Appendices 1 to 4.

The first patient group with AAA and no significant atherosclerotic arterial disease of the iliac or femoro-popliteal arteries comprised 46 patients of mean age $72.6 \mathrm{yrs}$ (range $40^{*}-88 \mathrm{yrs}$ ), mean AAA diameter $4.3 \mathrm{~cm}$ (range $3.0-6.4 \mathrm{~cm}$ ). 7 out of the 46 patients had aneurysmal disease of the iliac arteries in addition to an AAA and 2 patients had aneurysmal disease of the popliteal arteries in addition to an AAA. Because any changes to the CFA waveform with AAA may be influenced by the presence of additional aneurysmal disease this AAA group was divided into two sections: the first containing the 37 patients with an AAA and the second containing the 9 patients with an AAA and additional aneurysmal disease. The second patient group without AAA and no significant atherosclerotic arterial disease of the iliac or femoro-popliteal arteries comprised 41 patients of mean age 68.4yrs (range 50-92yrs), mean abdominal aorta diameter $1.6 \mathrm{~cm}$ (range $1.1-2.3 \mathrm{~cm}$ ).

\footnotetext{
* A 40 year old male presented with a $5.2 \mathrm{~cm}$ AAA and was included in the study. All other patients were aged over 50 years.
} 
In the first section of the research the waveforms from the first and second patient groups were studied and compared (as discussed in Chapter 3). A MatLab based computer program was then written to differentiate between the two groups (as discussed in Chapter 4). At the beginning of the study, 30 AAA patients (with no additional aneurysmal disease) and 30 patients without AAA were selected at random from the first and second patient groups respectively. These were used as 'study' groups to determine the differences between the AAA and normal CFA waveforms and to set up the AAA detection method. The remaining 7 AAA patients and 11 out of the 41 patients without AAA in the first and second groups were separated from the data sets. These were used as blind sets of test data which were used at the end of the study to test the success of the chosen AAA detection method and reduce bias. The remaining 9 patients with an AAA and additional aneurysmal disease were used a second blind test set at the end of the study.

The third patient group with AAA and significant atherosclerotic disease of the iliac and/or femoro-popliteal arteries comprised 24 patients of mean age 75.7yrs (range 55$91 \mathrm{yrs}$ ) and mean AAA diameter $4.9 \mathrm{~cm}$ (range $3.0-6.3 \mathrm{~cm}$ ). 1 out of these 24 patients had iliac aneurysms in addition to AAA. 6 out of these 24 AAA patients had significant proximal (iliac) disease. The forth patient group without AAA but with significant atherosclerotic disease of the iliac and/or femoro-popliteal arteries comprised 29 patients of mean age 72.6yrs (range 50-96yrs) and mean abdominal aorta diameter $1.7 \mathrm{~cm}$ (range $1.1-2.6 \mathrm{~cm}$ ). 7 of these 29 patients had significant proximal (iliac) disease. Because proximal and distal disease have different affects on the blood flow in the CFA, the patients with and without AAA in the significant 
atherosclerotic disease groups were sub-divided into those with distal (femoropopliteal) disease and those with proximal (aorto-iliac) disease.

In the second section of the research the waveforms from the third and forth patient groups were studied and compared (as discussed in Chapter 5). The MatLab based computer program was then used to differentiate between the two groups (as discussed in Chapter 6). At the beginning of the study, 15 AAA patients with significant distal disease and 18 patients without AAA with significant distal disease were selected at random from the third and forth and second patient groups respectively. These were used as 'study' groups to set up the AAA detection method. The remaining 3 out of the 18 AAA patients and 4 out of the 22 patients without AAA in the third and forth groups with significant distal disease were separated from the data sets. These were used as blind sets of test data which were used at the end of the study to test the success of the chosen AAA detection method and reduce bias.

Studies show that the prevalence of AAA is up to $7.6 \%$ in men and $1.3 \%$ in women (Scott, Wilson et al. 1995; Scott, Bridgewater et al. 2002) and the prevalence of atherosclerotic disease affecting the lower limb arteries is around $12 \%$ (Fowkes, Housley et al. 1991; Ostchega, Paulose-Ram et al. 2007). The relative sizes of the groups with and without AAA, and with and without significant aorto-iliac and or femoro-popliteal atherosclerotic disease in this study are not representative of the proportions in the general population. This was because it was necessary for the there to be enough patients in each category to facilitate analysis of reasonable sample sizes. 


\begin{tabular}{|c|c|c|c|c|c|c|c|c|c|}
\hline $\begin{array}{l}\text { Occlusive Disease } \\
\text { Category }\end{array}$ & $\begin{array}{c}\text { AAA } \\
\text { Category }\end{array}$ & $\begin{array}{c}\text { Total } \\
\text { Number } \\
\text { of } \\
\text { Patients }\end{array}$ & \multicolumn{2}{|c|}{ Group } & $\begin{array}{l}\text { Number } \\
\text { of Patients }\end{array}$ & $\begin{array}{c}\text { Mean Age } \\
\text { (yrs) }\end{array}$ & $\begin{array}{c}\text { Range } \\
\text { (yrs) }\end{array}$ & $\begin{array}{l}\text { Mean Abdominal } \\
\text { Aorta Diameter } \\
\text { (cm) }\end{array}$ & Range (cm) \\
\hline \multirow{5}{*}{$\begin{array}{l}\text { No Significant Aorto- } \\
\text { Iliac or Femoro- } \\
\text { Popliteal } \\
\text { Atherosclerotic } \\
\text { Disease }\end{array}$} & \multirow[b]{3}{*}{ AAA } & \multirow[b]{3}{*}{46} & \multicolumn{2}{|c|}{ Study Group } & 30 & 70.8 & $40-85$ & 4.6 & $3.2-5.8$ \\
\hline & & & \multicolumn{2}{|c|}{$\begin{array}{l}\text { Test group } \\
\text { Additional }\end{array}$} & 7 & 76.1 & $61-83$ & 3.9 & $3.1-5.2$ \\
\hline & & & \multicolumn{2}{|c|}{$\begin{array}{c}\text { Aneurysmal } \\
\text { Disease }\end{array}$} & 9 & 75.7 & $68-88$ & 4.3 & $3.0-5.7$ \\
\hline & \multirow{2}{*}{ No AAA } & \multirow{2}{*}{41} & \multicolumn{2}{|c|}{ Study Group } & 30 & 67.5 & $52-92$ & 1.6 & $1.1-2.2$ \\
\hline & & & \multicolumn{2}{|c|}{ Test group } & 11 & 70.9 & $50-83$ & 1.8 & $1.4-2.3$ \\
\hline \multirow{6}{*}{$\begin{array}{c}\text { Significant Aorto- } \\
\text { Iliac or Femoro- } \\
\text { Popliteal } \\
\text { Atherosclerotic } \\
\text { Disease }\end{array}$} & \multirow{3}{*}{ AAA } & \multirow{3}{*}{24} & \multirow{2}{*}{$\begin{array}{l}\text { Distal } \\
\text { Disease }\end{array}$} & $\begin{array}{l}\text { Study } \\
\text { Group }\end{array}$ & 15 & 73.9 & $55-85$ & 4.6 & $3.0-6.0$ \\
\hline & & & & $\begin{array}{c}\text { Test } \\
\text { Group }\end{array}$ & 3 & 85 & $76-91$ & 5.1 & $4.8-5.6$ \\
\hline & & & $\begin{array}{c}\text { Proximal } \\
\text { Disease }\end{array}$ & $\begin{array}{l}\text { Study } \\
\text { Group }\end{array}$ & 6 & 75.3 & 73-79 & 5.0 & $3.6-6.3$ \\
\hline & \multirow{3}{*}{ No AAA } & \multirow{3}{*}{29} & \multirow{2}{*}{$\begin{array}{c}\text { Distal } \\
\text { Disease }\end{array}$} & $\begin{array}{l}\text { Study } \\
\text { Group }\end{array}$ & 18 & 74.2 & $50-96$ & 1.6 & $1.3-2.2$ \\
\hline & & & & $\begin{array}{c}\text { Test } \\
\text { Group }\end{array}$ & 4 & 70.3 & $63-75$ & 1.6 & $1.3-2.0$ \\
\hline & & & $\begin{array}{c}\text { Proximal } \\
\text { Disease }\end{array}$ & $\begin{array}{l}\text { Study } \\
\text { Group }\end{array}$ & 7 & 69.7 & $51-87$ & 1.8 & $1.2-2.6$ \\
\hline
\end{tabular}

Table 2.2: Patient data and group summaries 
Chapter 3: Visual Analysis of CFA Waveforms from AAA and Normal Patients with No Significant Atherosclerotic Disease

\section{$\underline{\text { 3.1 Introduction and Aim }}$}

Routine visual examination of CFA waveforms during routine clinical studies indicated that when certain changes were observed in the CFA Doppler waveform, an AAA was often present on examination of the distal aorta. Following the review of the literature in Chapter 1, these changes seemed to be consistent with certain features of flow within AAA models and also with waveform changes observed in vivo by Humphries et al. A preliminary study, described in this chapter, was performed to investigate these observations more formally in patients with no significant aorto-iliac or femoro-popliteal atherosclerotic disease. The aim was to determine the type of changes to the CFA Doppler waveform that may be associated to AAA, and to determine the feasibility of detecting AAA by visual identification of CFA waveform features.

\subsection{Method}

The patient cohort comprised the 30 AAA patients and 30 normal patients in the study groups with no significant femoro-popliteal atherosclerotic disease (summarised in Table A1.1, Appendix 1 and Table A2.1, Appendix 2). For each patient the CSV data files for the left and right CFA waveforms measured using the Xario-DopStudio system were reproduced as spectrograms in MATLAB using the 'imagesc' plotting function in the 'vga' colour scale. 
The waveforms within the spectrograms were visually inspected to identify features within the waveform cycles which differed from 'ideal' CFA waveform cycles as shown in Figure 3.1. These 'ideal' waveform cycles are triphasic with uniform upstroke and down-stroke and minimal spectral broadening.
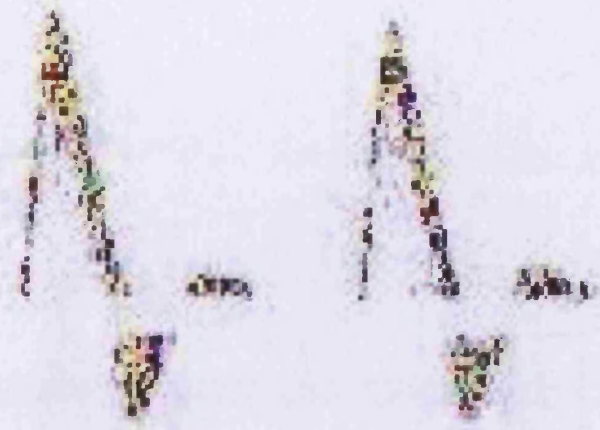

Figure 3.1: Normal 'ideal' CFA waveform cycles

\subsection{Feature Identification}

On visual inspection of the CFA waveforms 5 features were noted, predominantly in the AAA group, as being different from an 'ideal' CFA waveform: 1) spectral broadening on the systolic down-stroke, 2) the appearance of 'spikes' on the systolic down-stroke, 3) an irregular reverse flow pattern, 4) simultaneous forward and reverse flow during systole and 5) elongation of the waveform during the cardiac cycle.

Examples of the first feature, that of increased spectral broadening in the deceleration phase of the systolic peak in waveform cycles from AAA and normal patients, are shown in Figures 3.2 and 3.3 respectively. In the presence of atherosclerotic arterial 
disease, spectral broadening increases but is generally exhibited as an in-fill under the curve of the systolic peak. None of the patients in these study groups had significant atherosclerotic arterial disease. In the normal patient waveforms, the spectral broadening is generally exhibited as an infill under the peak, whereas in the AAA waveforms the increase in spectral broadening is mainly in the systolic down-stroke region.
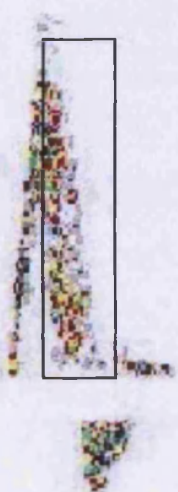

a)

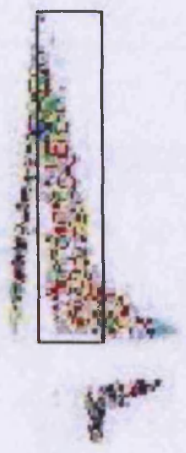

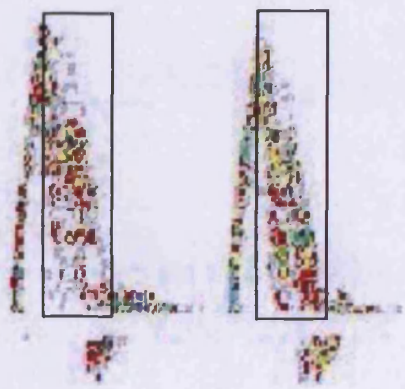

b)

Figure $3.2 \mathrm{a}$ and b: Examples of spectral broadening on the systolic down-stroke in AAA waveform cycles

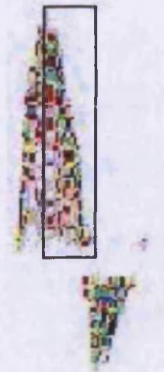

a)

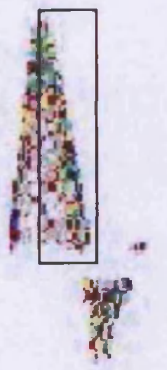

in

Figure $3.3 \mathrm{a}$ and $\mathrm{b}$ : Examples of spectral broadening on the systolic down-stroke in normal waveform cycles 
The spectral broadening observed in many of the AAA waveforms had a spiked appearance extending both above and below the downward slope of the systolic peak. In some cases the 'spikes' also extended down into the reverse flow region. Examples of this feature of spikes in the spectral broadening on the systolic down-stroke in waveform cycles from AAA and normal patients are given in Figures 3.4 and 3.5 respectively. In general, the spikes were more prominent in the AAA patient waveforms.
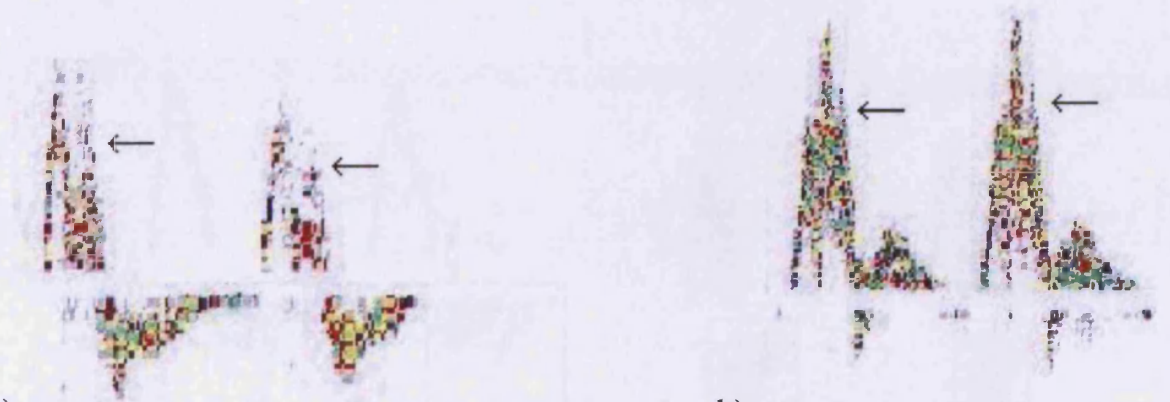

a)

b)

Figure $3.4 \mathrm{a}$ and $\mathrm{b}$ : Examples of spikes on the systolic down-stroke in AAA waveform cycles

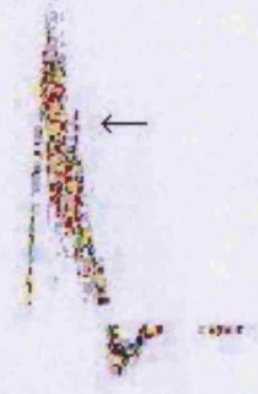

a)

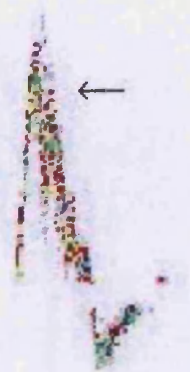

Figure $3.5 \mathrm{a}$ and $\mathrm{b}$ : Examples of spikes on the systolic down-stroke in normal waveform cycles 
Examples of the third feature of an irregular reverse flow pattern observed in some of the waveform cycles from AAA patients are shown in Figure 3.6. There were two types of irregularity noted in the reverse flow. The first, shown in Figure 3.6a illustrates an elongation of the reverse flow component with an irregular profile and distinct spikes. The second, shown in Figure 3.6b illustrates an 'exaggerated' reverse flow component, with increased amplitude and a slightly square, irregular profile. Examples of these irregular reverse flow patterns observed in waveform cycles from normal patients are given in Figure 3.7.

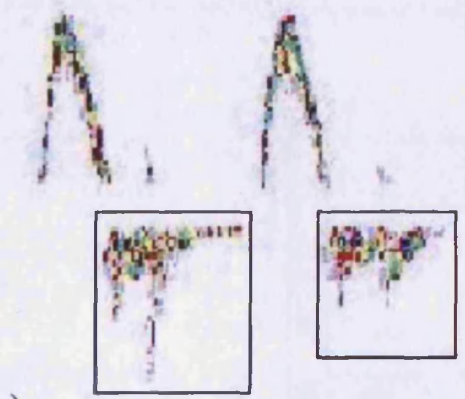

a)

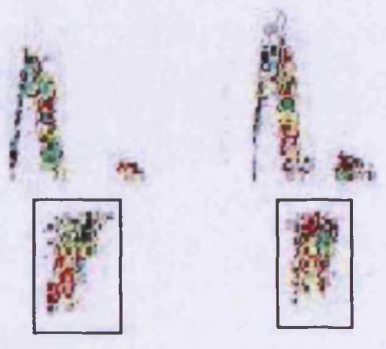

b)

Figure $3.6 \mathrm{a}$ and $\mathrm{b}$ : Examples of irregular reverse flow patterns in AAA waveform cycles

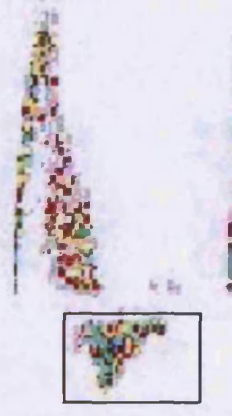

a)

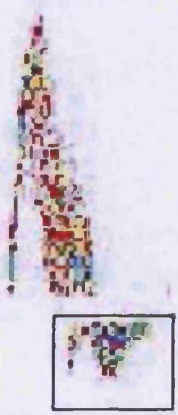

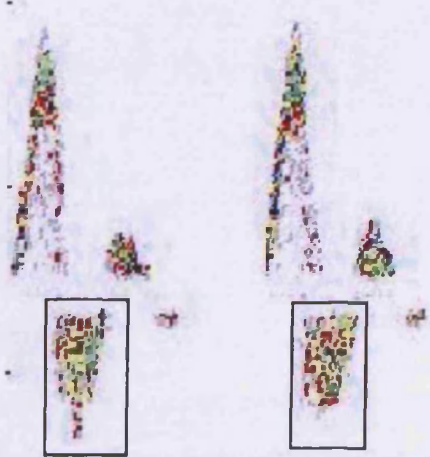

b)

Figure $3.7 \mathrm{a}$ and $\mathrm{b}$ : Examples of irregular reverse flow patterns in normal waveform cycles 
An example of waveform cycles from an AAA patient displaying the fourth feature of simultaneous forward and reverse flow under the systolic peak is given in Figure 3.8. In the 'ideal' CFA waveform cycle the reverse flow component occurs after the systolic peak as a result of pressure reflection and elastic recoil of the artery wall. In this feature low power reverse flow can be seen under the systolic peak before the onset of the 'true' reverse flow component. This can possibly be attributed to flow disturbances occurring during the systolic flow phase. An example of this feature seen in waveform cycles from a normal patient is given in Figure 3.9.

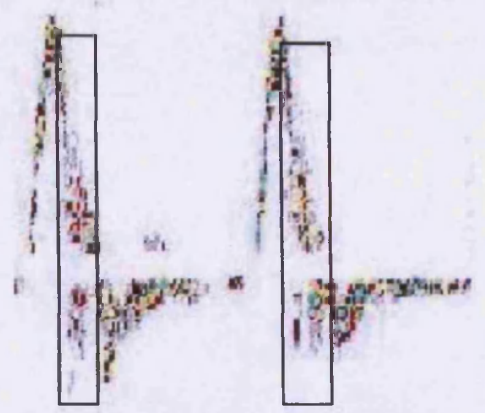

Figure 3.8: Examples of simultaneous forward and reverse flow in AAA waveform cycles
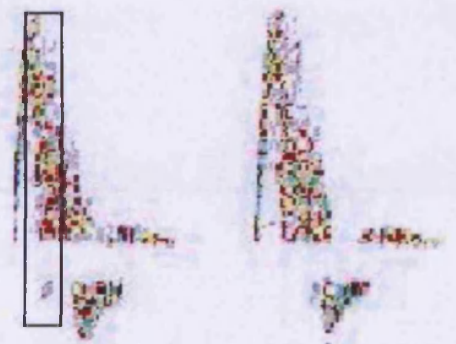

Figure 3.9: Examples of simultaneous forward and reverse flow in normal waveform cycles 
The fifth feature noted in the waveforms was a subtle elongation of the waveform cycle length within the beat cycle. An example of waveform elongation in waveform cycles from AAA and normal patients are given in Figures 3.10 and 3.11 respectively. Typically, with this feature the waveform cycle appeared to be 'spread out' with an additional reverse flow phase extending over the rest of the cardiac cycle.

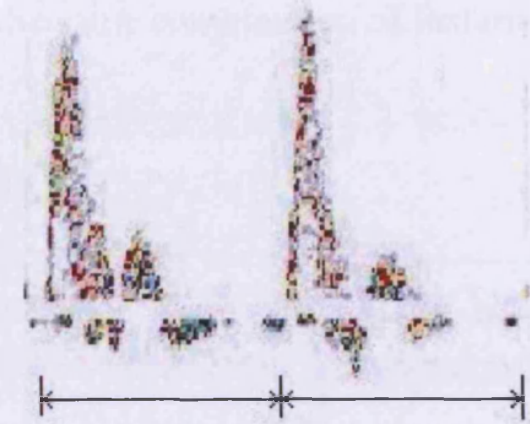

Figure 3.10: Example of waveform elongation in AAA waveform cycles

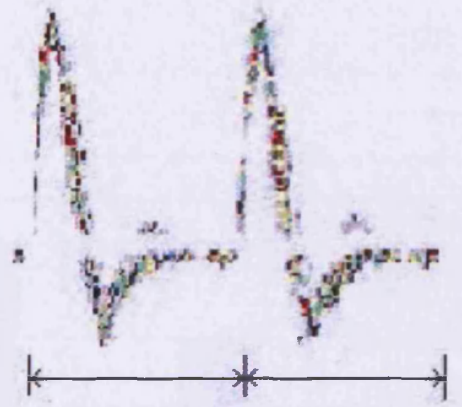

Figure 3.11: Example of waveform elongation in normal waveform cycles

\subsection{AAA Detection Using CFA Visual Feature Analysis}

In order to determine the feasibility of detecting AAA using visual feature analysis, the waveforms from the $30 \mathrm{AAA}$ and 30 normal patients were scored according to the presence of the 5 features. It is interesting to note that in both patient groups, the CFA waveforms from the right and left legs in the same patient did not necessarily exhibit 
the same features. This may possibly be due to slight differences between the paths of the iliac arteries on the left and right side. Different levels of tortuosity and minor calcification between the two sides may also possibly explain the differences. Therefore the left and right CFA waveforms for each patient were individually scored according to the 5 features. If a feature was present in one or more cycles within the waveform, a score of 1 was awarded for that feature. Only 3 AAA patients and 2 normal patients exhibited the same combination of features in the waveforms from the left and right legs.

\subsubsection{Waveform Feature Results}

Figure 3.12 shows the number of CFA waveforms out of the $60 \mathrm{AAA}$ and 60 normal waveforms with each feature present.

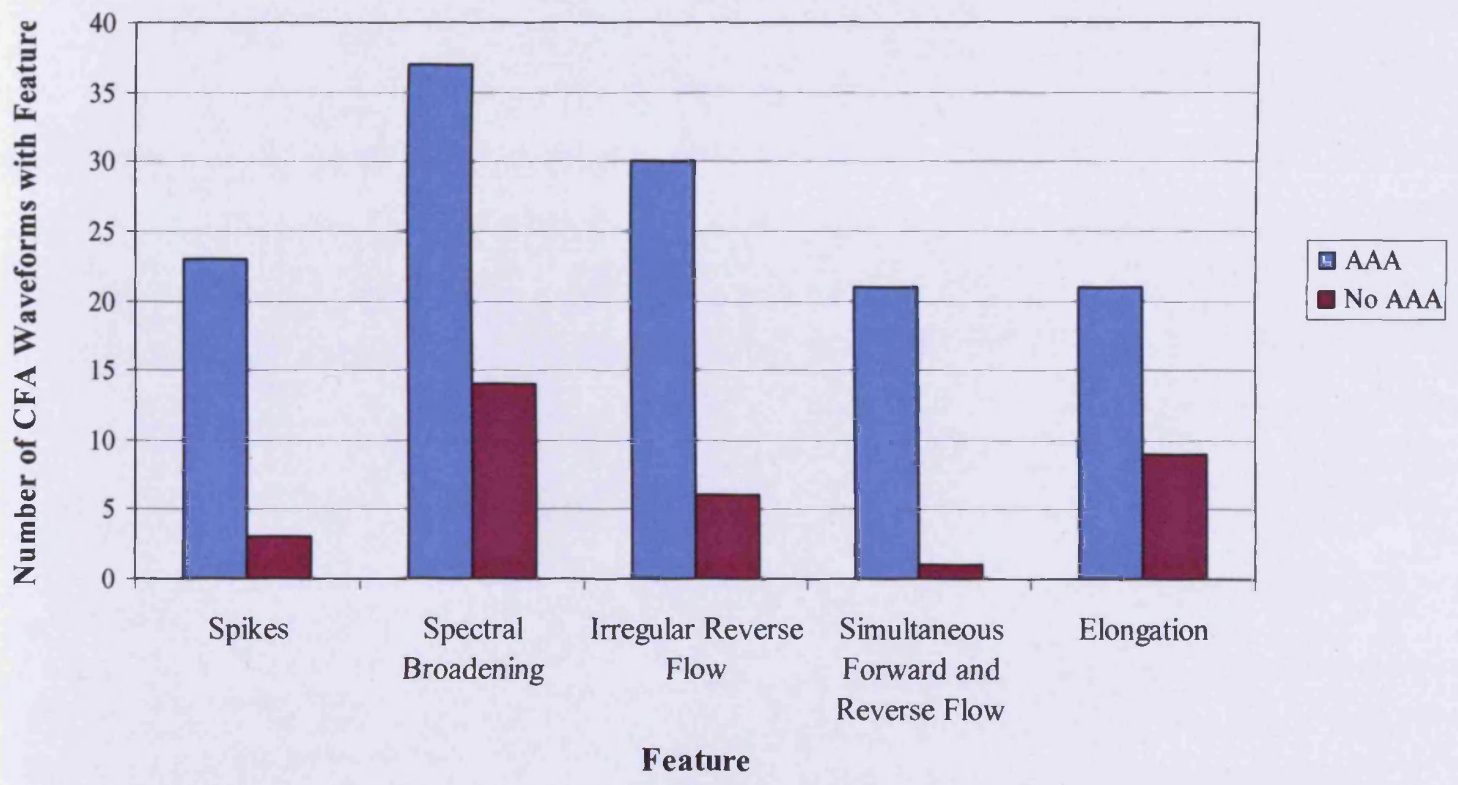

Figure 3.12: Number of CFA waveforms from AAA and normal patients in the no significant arterial disease study groups with each feature present visually 


\subsubsection{Waveform Feature Results: Discussion}

All 5 features provided good differentiation between AAA and normal waveforms. The two most prominent features in the AAA group were spectral broadening and irregular reverse flow, but there was no single feature which was characteristic to all AAA waveforms. Therefore for each waveform the scores for all 5 factors were combined in order to improve the differentiation between the two groups.

\subsubsection{Individual Patient Scores}

Because the left and right CFA waveforms for a patient often contained different features the total factor score for an individual patient was based on the summation of the total scores of the left and right leg CFA waveforms.

\section{$\underline{3.4 .4 \text { Individual Patient Score Results }}$}

The total scores for the 30 AAA and 30 normal patients are plotted in Figures $3.13 \mathrm{a}$ and $3.13 b$.

The total patient scores for the 30 AAA and 30 normal patients were then used to set a threshold level to best distinguish between the AAA and normal groups. Figure 3.14 shows the number of $\mathrm{AAA}$ and normal patients with a total patient score of $\geq 1, \geq 2$ and $\geq 3$. 


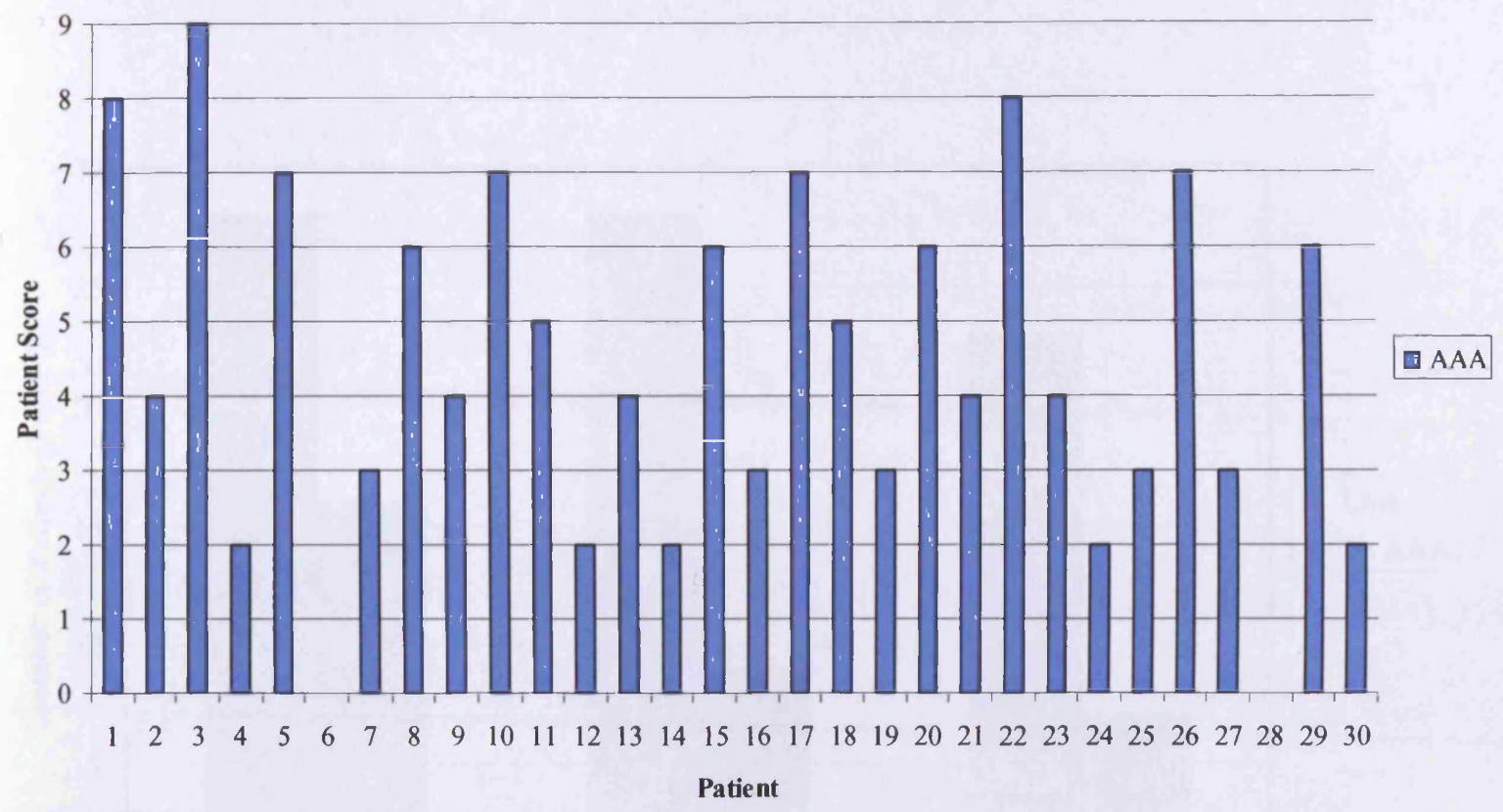

Figure 3.13a: Total Patient Scores for the 30 AAA patients in the study group with no significant atherosclerotic disease

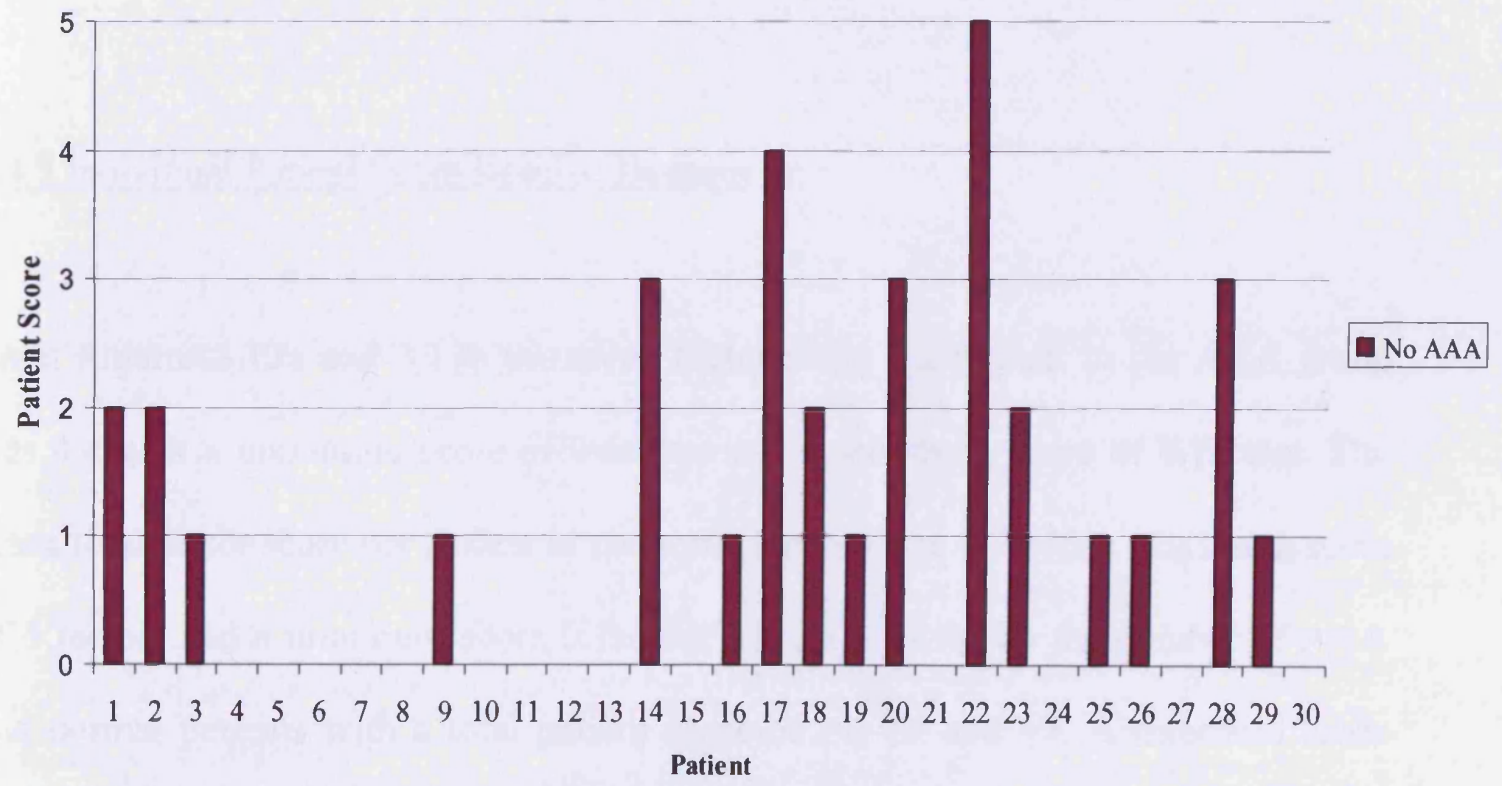

Figure 3.13b: Total Patient Scores for the 30 normal patients in the study group with no significant atherosclerotic disease 


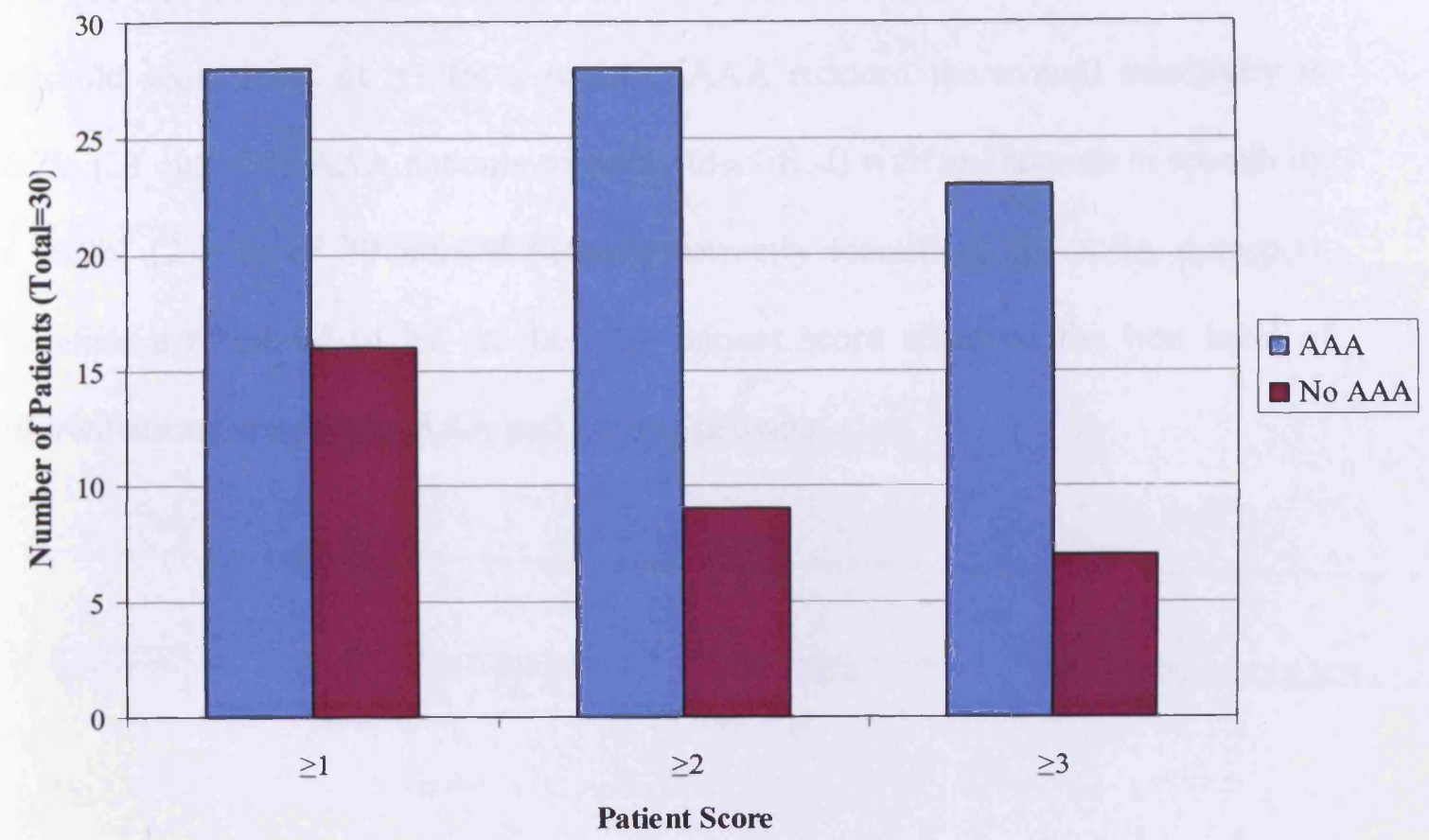

Figure 3.14: Number of AAA and normal patients in the study groups with no significant arterial disease with total score $\geq 1, \geq 2$ and $\geq 3$

\subsubsection{Individual Patient Score Results: Discussion}

From Figures $3.13 \mathrm{a}$ and $3.13 \mathrm{~b}$ the mean factor score per patient in the AAA group was 4.4 with a maximum score of 9 factors and a minimum score of 0 factors. The mean total factor score per patient in the normal group was 1.1 with a maximum score of 5 factors and a minimum score 0 factors. Figure 3.14 shows the number of AAA and normal patients with a total patient score of $\geq 1, \geq 2$ and $\geq 3$. A threshold score level of $\geq 1$ for a positive AAA, achieved an overall sensitivity of $93.3 \%$ ( 28 out of 30 AAA patients correctly identified) with a specificity of $47 \%$ (14 out of 30 normal 
patients correctly identified) for AAA detection. The threshold score level of $\geq 2$ for a positive AAA also provided a sensitivity of $93.3 \%$ with an improved specificity of $70 \%$ (21 out of 30 normal patients correctly identified) for AAA detection. The threshold score level of $\geq 3$ for a positive AAA reduced the overall sensitivity to $76.7 \%$ (23 out of 30 AAA patients correctly identified) with an increase in specificity to $76.7 \%$ (23 out of 30 normal patients correctly identified) for AAA detection. Therefore a threshold of $\geq 2$ on the total patient score achieved the best level of differentiation between the AAA and normal patients. 


\section{$\underline{\text { 3.5 Discussion }}$}

In this preliminary study of 30 patients with AAA and 30 patients without AAA with both groups having no significant femoro-popliteal arterial disease, there were 5 features noted on visual inspection of the CFA waveforms which differed from an 'ideal' CFA waveform, and that were more predominant in the AAA patient group: 1) 1) spectral broadening on the systolic down-stroke, 2) the appearance of spikes on the systolic down-stroke, 3) an irregular reverse flow pattern, 4) simultaneous forward and reverse flow during systole and 5) elongation of the waveform during the cardiac cycle.

To our knowledge, the effect of AAA on the CFA waveform has not been widely studied with only one previously reported study, that by Humphries et al. As far as we are aware there is no accepted explanation or suggestion as to why the 5 features observed in this study occur. The modelling studies discussed in Chapter 1 provide an insight into the flows occurring within aneurysms but do not examine the effects on the distal flow in the iliac or femoral arteries. Therefore, one can only hypothesise as to why these flow changes in the CFA exist. From the results of these modelling studies which suggest that flow disturbances with vortices and turbulence are set up within aneurysms, it is hypothesised that these flow disturbances may be transported distally and may manifest in the CFA waveform as the features described above.

The feature of spectral broadening may be explained by the increased range of Doppler frequencies resulting from the different orientations and magnitudes of 
velocity vectors of flow disturbances originating within the AAA remaining within the bulk flow in the CFA.

The features of spikes, irregular reverse flow and simultaneous forward and reverse flow may possibly be explained by considering the range of Doppler shift frequencies detected from a vortex recirculating within a Doppler ultrasound beam as illustrated in Figure 3.15.
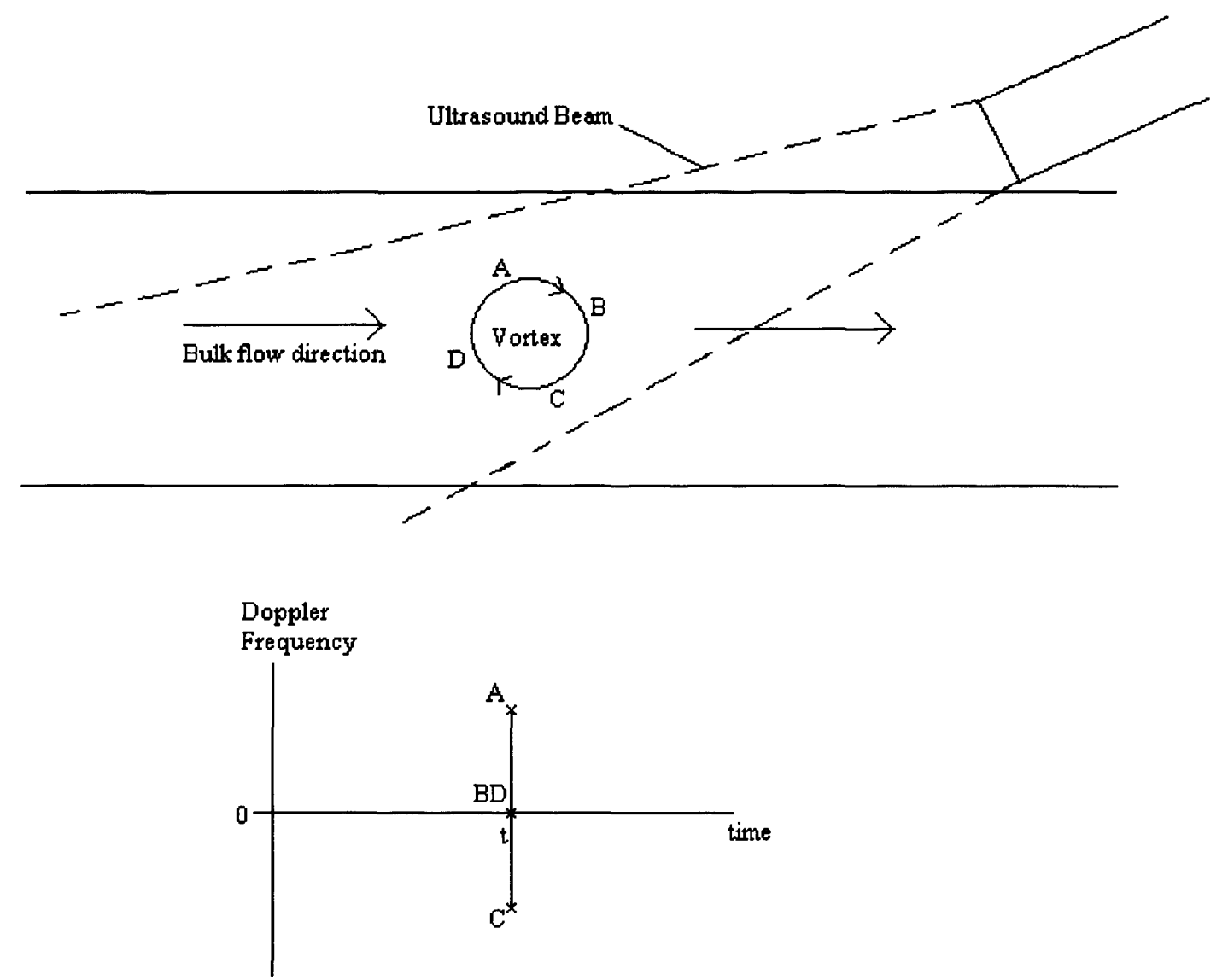

Figure 3.15: Doppler frequencies detected at a point in time from a recirculating vortex moving with the bulk flow within a Doppler beam 
By considering the Doppler shift frequencies detected at a point in time from points $\mathrm{A}, \mathrm{B}, \mathrm{C}$ and $\mathrm{D}$ on the vortex in this diagram during the forward flow phase, it can be seen that the Doppler shift from the vortex flow at point $A$ is maximum because the velocity vector is parallel to the ultrasound beam, directed towards the transducer producing a maximum, positive Doppler frequency shift. At point $\mathrm{C}$ the velocity vector is parallel to the Doppler beam but opposed to the transducer, producing a maximum negative Doppler frequency shift. At points B and D the velocity vectors of the vortex flow are perpendicular to the beam direction resulting in zero Doppler shift. If the Doppler frequencies corresponding to the velocity vectors of the vortex motion are displayed on a spectrogram, at a single point in time there will be a continuum of frequencies ranging from the maximum negative frequency up to the maximum positive frequency. The magnitude of the maximum positive and negative Doppler shifts detected from the vortex motion are the same, however when these are superimposed on the normal flow profile, the range of frequencies are off set in the positive direction due to the translation of the vortex within the bulk flow towards the transducer.

The modelling studies suggest that vortices and flow disturbances are set up within an aneurysm during flow deceleration. The appearance of spikes on the systolic downstroke of the CFA waveform may therefore correspond to the motion of vortices and flow disturbances set up within the bulk flow during flow deceleration in the systolic flow phase. The feature of simultaneous forward and reverse flow during the systolic flow phase may also possibly be explained by the motion of vortices within the bulk flow: the bulk flow direction is forwards but there will also be velocity vectors in the reverse flow direction detected from the circulating flow regions. The irregularities in 
the reverse flow profile may also possibly be explained in a similar manner as described above for the forward flow phase.

If the flow disturbances set up within AAAs are transported within the bulk flow into the femoral vessels, this may well explain the increased spectral broadening and spikes in both the forward and reverse flow phases. The feature of elongation is less easily explained. The reverse flow phase results form elastic recoil of the artery wall, therefore elongation of the reverse flow phase may be in some way related to the elastic recoil properties of the AAA wall and the corresponding development of flow. However, further research is necessary, possibly using numerical fluid dynamics modelling with finite element analysis techniques to simulate the development, decay and transportation of AAA flow disturbances through the iliac and femoral vessels to fully understand the haemodynamics and explain the features observed in this study.

By scoring the CFA waveforms according to the presence of these 5 features and setting a threshold of $\geq 2$ on the sum of the left and right CFA waveform scores for each patient, it was possible to predict AAA in patients with no significant femoropopliteal arterial disease with a sensitivity of $93.3 \%$ and a specificity of $70 \%$. These results suggest it is feasible to detect AAA by analysis of the CFA waveform.

Visual inspection of CFA waveforms is subjective, especially because the waveform changes can be subtle. Arterial Doppler waveforms are affected by several different factors and although each artery in the body has a 'signature' waveform, even in the absence of any aneurysmal or atherosclerotic disease the waveform characteristics vary from person to person depending on several inherent factors; for example, 
cardiac output, vessel size and tortuosity. Because visual examination is so subjective there will be a certain level of bias in determining whether waveform changes are present or not. In the absence of any significant occlusive arterial disease, 28 out of the 30 waveforms do show distinct waveform changes which may possibly be attributed to flow disturbances from an AAA. Therefore, a much more objective analysis method is necessary to reduce bias. An approach would be to extract the features more objectively using MATLAB software based analysis.

Visual inspection of arterial waveforms is not only subjective, but is also largely limited to the interpretation of waveform shapes. There may also be changes to aspects of the waveforms not obvious even to a trained eye. The Doppler spectrogram is a 2-D representation of a 3-D data set with the time versus frequency-shift on the $\mathrm{x}$ $\mathrm{y}$ axis and the power of each frequency component at a point in time proportional to the brightness of each pixel within the spectrogram. Other features such as the distribution of frequency components and corresponding intensities may also differ between AAA and normal patients. It may be possible to identify such features by implementing more sophisticated analysis using MATLAB software.

It is accepted that the sensitivity and specificity sensitivity of $93.3 \%$ and a specificity of $70 \%$ achieved with these visual inspections are biased because the results are from on a single trained observer analysing non-blinded data subjectively. However, the main aim of the work in this chapter was to identify waveform features. The sensitivity and specificity were only calculated to determine whether it was worth pursuing more detailed, objective MatLab based analysis. In conclusion, it may be feasible to objectively identify features characteristic to AAA and objectively 
diagnose AAA from CFA waveforms using MATLAB software. The following chapter describes the next stage of the research in which a MATLAB program was designed and tested to try and improve on the results achieved in the visual analysis for AAA detection by objective analysis of the CFA waveforms based on the 5 features identified in this preliminary study. Bias was reduced by the objective nature of the MatLab analysis and by subjecting the program to blind data sets to verify the results. 


\section{Chapter 4: AAA Detection by Objective Analysis of CFA Waveforms in Patients}

with No Significant Atherosclerotic Disease

\subsection{Introduction and Aim}

Visual analysis of CFA waveforms from patients with and without AAA with no significant iliac or femoro-popliteal atherosclerotic disease in Chapter 3 identified five features: 1) spectral broadening on the systolic down-stroke, 2) the appearance of 'spikes' on the systolic down-stroke, 3) an irregular reverse flow pattern, 4) simultaneous forward and reverse flow during systole and 5) elongation of the waveform during the cardiac cycle which were different from the features of an 'ideal' waveform and which were more prominent in waveforms from patients with an AAA than from normal patients. Based on visual identification of these features it was possible to detect AAA in the 30 AAA patients compared with the 30 normal patients in the no significant atherosclerotic disease study groups with a sensitivity of $93.3 \%$ and a specificity of $70 \%$. Visual analysis of Doppler waveforms is subjective and requires a high level of training. Therefore the aim of the work in this chapter was to develop a method to automatically and objectively analyse the CFA waveforms to detect AAA based on the 5 the features noted in the visual analysis. The following sections describe the method by which a MATLAB based program capable of detecting AAA from CFA waveforms in patients with no significant femoropopliteal atherosclerotic disease was written and tested. 


\subsection{Method Overview}

The CFA waveforms from the $30 \mathrm{AAA}$ and 30 normal patients in the study groups with no significant iliac or femoro-popliteal atherosclerotic disease, that were visually analysed in Chapter 3 were used to set up a MATLAB based AAA detection program. In the first stage of the work, described in section 4.3 , initial data processing was performed using MATLAB code to manipulate and interrogate the CFA waveform data. The aim was to extract parameters from which the five waveform features noted in the visual inspections in Chapter 3 could be described. In the second stage, discussed in section 4.4 , these parameters were used to calculate a number of indices to provide measures for each of the 5 features. In the third stage, described in section 4.5 , a range of threshold levels were tested on each of the indices to determine the index levels which provided the best discrimination between AAA and normal waveforms in the no significant atherosclerotic arterial disease study groups. In section 4.6, the indices were used to set up a MATLAB based AAA detection program. The selected indices were tested in a range of different combinations, with a number of different threshold levels and weighting factors applied, to select the permutation providing the best sensitivity and specificity for AAA detection in the 30 AAA and 30 normal study group patients. In section 4.7, the performance of the detection program was tested by its response to the waveform data from the AAA and normal patients with no significant atherosclerotic arterial disease that were placed into the test groups at the start of the study. In section 4.8 , the performance of the program in response to the CFA waveforms measured from the group of patients with AAA and additional aneurysmal disease of the iliac or popliteal arteries was assessed. The AAA detection program performance and results are discussed in section 4.9. 


\subsection{Initial Data Processing}

The CSV files containing the CFA waveform data were loaded into the MATLAB software and stored as 256 by 500 matrices. Initial data processing was performed on the waveform data with two main aims: 1) to reduce noise in the data and 2) to identify reference points within individual waveform (cardiac) cycles. Both these aims were achieved with programming based on the calculation of a parameter called the maximum frequency envelope.

\subsubsection{Extraction of the Maximum Frequency Envelope}

The maximum frequency envelope (MFE) is commonly used in the processing of Doppler ultrasound signals. It is integral to several well documented analysis methods for the calculation of diagnostic indices such as the Pulsatility Index. It is also valuable for the identification of regions of interest within the signal, for example individual cardiac cycles. The MFE is derived from the amplitude of the Doppler frequency spectrum. For this study the MFEs were extracted using the 95 percentile method. In this method the amplitudes of the pixels in each column of the data matrix (corresponding to a point in time on the spectrogram) were summed, and the maximum frequency point was calculated as the pixel that corresponded to $95 \%$ of the summed frequency amplitudes at this point in time. The $95 \%$ level was chosen in order to eliminate spurious noise in the spectrogram lying outside the true arterial signal. The method does not distinguish between positive and negative frequency shifts and therefore the MFEs were calculated separately for the forward and reverse flow components of each waveform. An example of an MFE generated for the 
forward flow components of a normal CFA waveform using this method is shown in Figure 4.1. The MFE can be affected by noise in the signal. Therefore the MFE was subjected to a $6^{\text {th }}$ order Butterworth low-pass filter to remove the unwanted high frequency noise components. The resulting MFE was cleaner as shown in Figure 4.2.

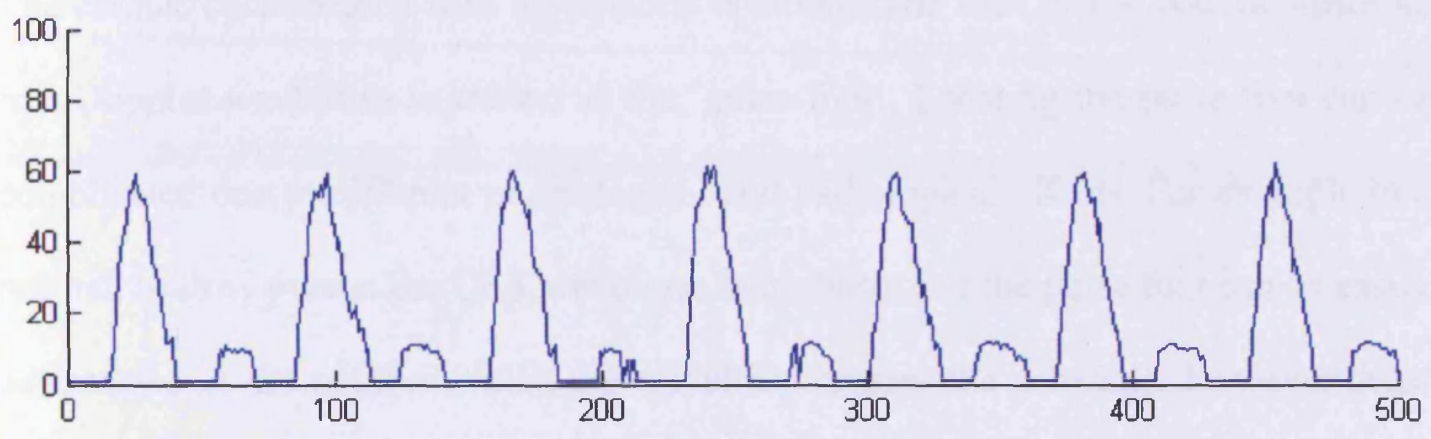

Figure 4.1: Unfiltered MFE

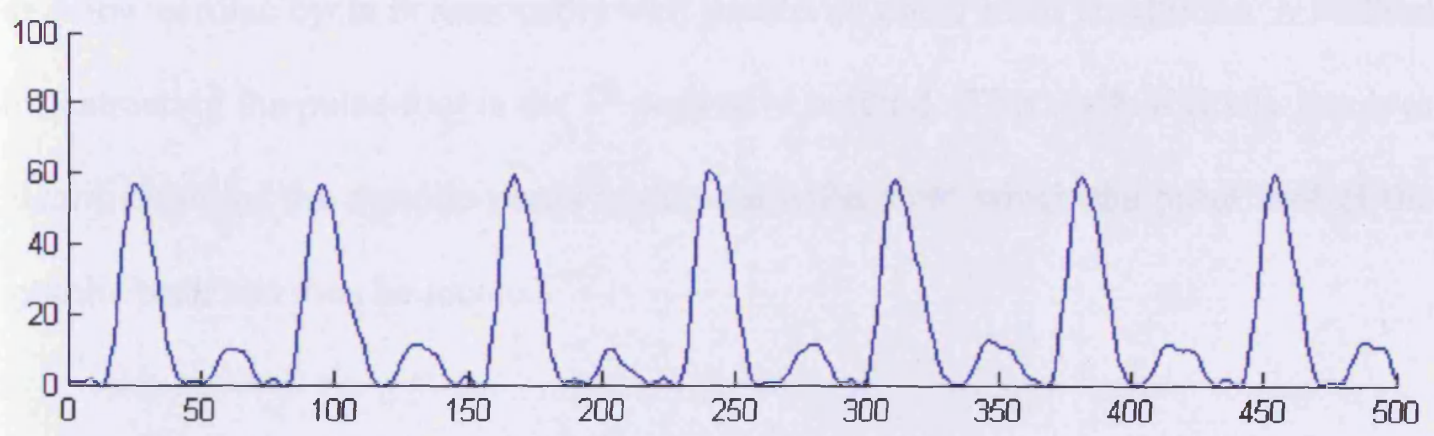

Figure 4.2: Butterworth filtered MFE 


\subsubsection{Identification of Individual Waveform Cycles}

Blood flow patterns usually contain specific repeating features corresponding to the phases of the cardiac cycle. The filtered MFEs were used to locate and extract fixed reference points within the spectrogram for example the start and end of each cardiac (waveform) cycle. Once individual waveform cycles were isolated, it was possible to extract specific regions of interest within or between each pulse.

The cardiac cycle begins with the systolic upstroke. The start of the systolic upstroke in a Doppler waveform is known as the 'pulse foot'. Locating the pulse foot can be complicated due to different physiological and pathological effects. For example in a normal, healthy person the CFA waveform is triphasic and the pulse foot can be easily determined as the point at which the waveform crosses the zero line. However there may be no zero crossing in cases where there is vaso-dilation or significant disease causing very damped flow. Irregular heartbeats in conditions such as atrial fibrillation (AF) also introduce uncertainties. However, although difficult to locate, the pulse-foot of a new cardiac cycle is reasonably well preserved under most conditions. A method for extracting the pulse-foot is the $1^{\text {st }}$ derivative method. This method firstly involves identification of the systolic peaks within the MFE from which the pulse foot of the systolic peak can then be located.

\subsubsection{Identification of the Systolic Peak}

The systolic peaks were located by identifying all points on the systolic upstroke with amplitude $>40 \%$ of the maximum amplitude in the filtered MFE and then counting 
'up' this envelope matrix vector until the peak was reached. A threshold of 20 points was set between peak locations to prevent the increased amplitudes of spikes or shoulders in the systolic down-stroke being incorrectly assigned as a systolic peak.

To locate the pulse foot at the start of the systolic peak, the differentials between successive points 'down' the filtered MFE on the systolic upstroke were calculated and then interrogated to locate the pulse foot, which was defined as the first point of inflection of the differential values (where the differential value changed from negative to positive). This was repeated for all peaks located in the signal, except for peaks occurring within the first 20 points of the envelope in order to exclude incomplete peaks.

To locate the foot of the deceleration phase (down-stroke) of the systolic peak, the differentials of 60 subsequent, adjacent points along the filtered MFE from the peak along the down-stroke were calculated. The differential values were then interrogated to locate the end pulse foot, which was defined as first the point of inflection below a level of $50 \%$ of the envelope value at the systolic peak. The $50 \%$ threshold level was set to prevent 'spikes' or deflections in the systolic down-stroke being incorrectly identified as a pulse foot. The pulse foot of the deceleration phase was not calculated for the last peak in the spectrogram in case the peak was incomplete. Examples of the peak and pulse foot locations identified on the MFE using this method are shown in Figure 4.3. 


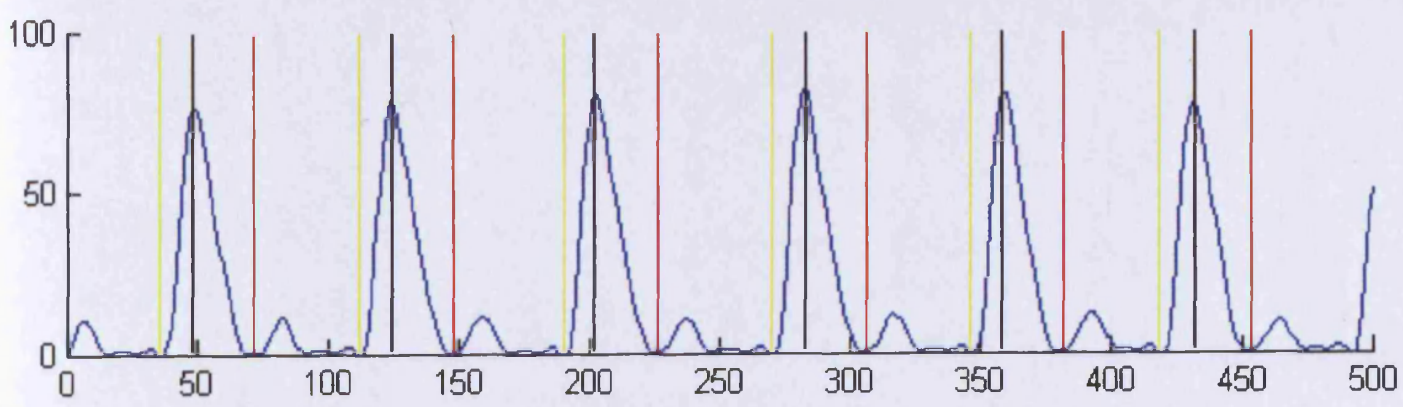

Figure 4.3: MFE with black, yellow and red lines indicating the peak, pulse foot of the upstroke and deceleration phase foot locations of the systolic peak respectively

\subsubsection{Noise Reduction}

During the measurements of the CFA waveforms, the gains on the Xario and DopStudio systems were adjusted to visually optimise the signal to noise ratio in each of the spectrograms. However, in some cases the background noise level remained high, for example in the case of low lying or calcified vessels. The 95 percentile method for MFE generation produced satisfactory MFEs, representative of the CFA waveform profile for spectrograms with low levels of background noise, but produced unsatisfactory results when waveforms with higher levels of background noise were subjected as shown in Figure 4.4. It was therefore necessary to reduce the unwanted background noise in the data to enable analysis of the true CFA signal.

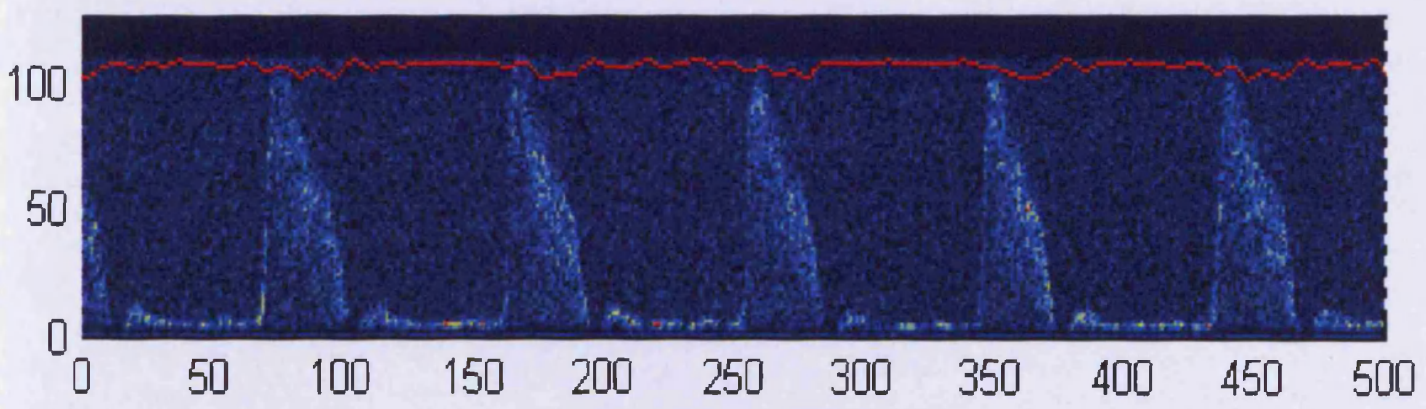

Figure 4.4: Unsatisfactory MFE (indicated by red line) for spectrogram containing a high level of background noise 


\subsubsection{Thresholding}

A thresholding technique known as 'grass cutting' was implemented to remove the unwanted low power background noise from the spectrogram. In this technique a threshold was set as a percentage of the maximum power amplitude in the signal and all pixels in the spectrogram matrix with an amplitude below this level were set to zero. Initially the threshold level was set to $10 \%$ of the maximum signal amplitude. This threshold level produced good results in general for cases of low background noise and more satisfactory MFEs were obtained as shown in Figure 4.5a. However, the $10 \%$ threshold still produced unsatisfactory results when signals with higher levels of background noise were subjected as shown in Figure 4.5b.

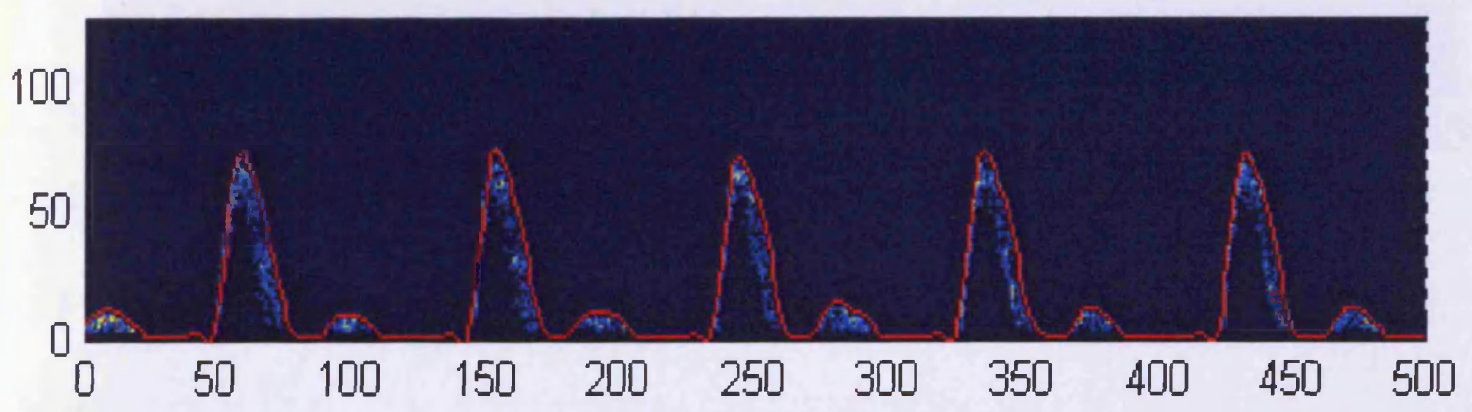

Figure 4.5a: MFE (indicated by red line) for a spectrogram containing a low level of background noise with $10 \%$ filter threshold applied

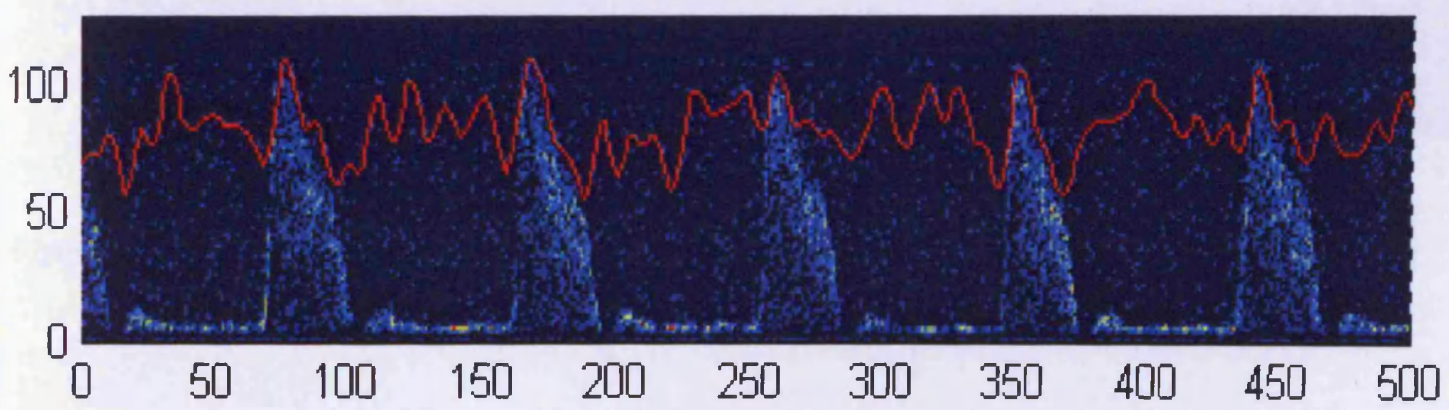

Figure 4.5b: MFE (indicated by red line) for a spectrogram containing a high level of background noise with $10 \%$ filter threshold applied 
A range of threshold levels were tested with the aim of determining an optimum threshold level. Higher threshold levels produced good results for noisy signals, for example Figure 4.6a shows the resulting MFE achieved with a $20 \%$ filter threshold applied to the spectrogram that was shown in Figure 4.4. However, it can be seen in Figure $4.6 \mathrm{~b}$ that when the $20 \%$ filter threshold was applied on the same spectrogram as shown in Figure 4.5a, a crude MFE was produced but too much spectral data was removed from the true arterial signal.

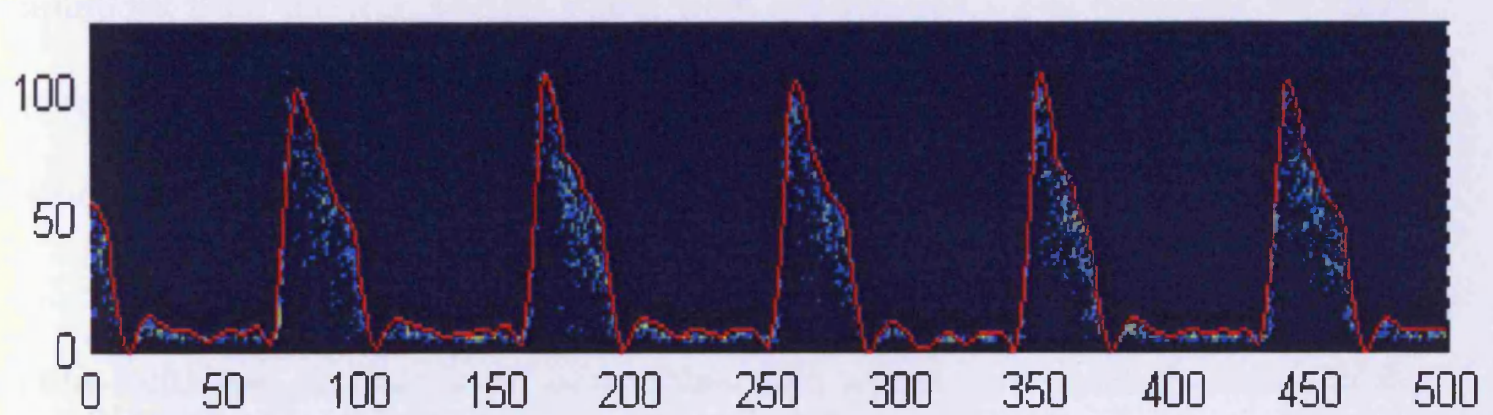

Figure 4.6a: MFE (indicated by red line) for a spectrogram containing a high level of background noise with $20 \%$ filter threshold applied

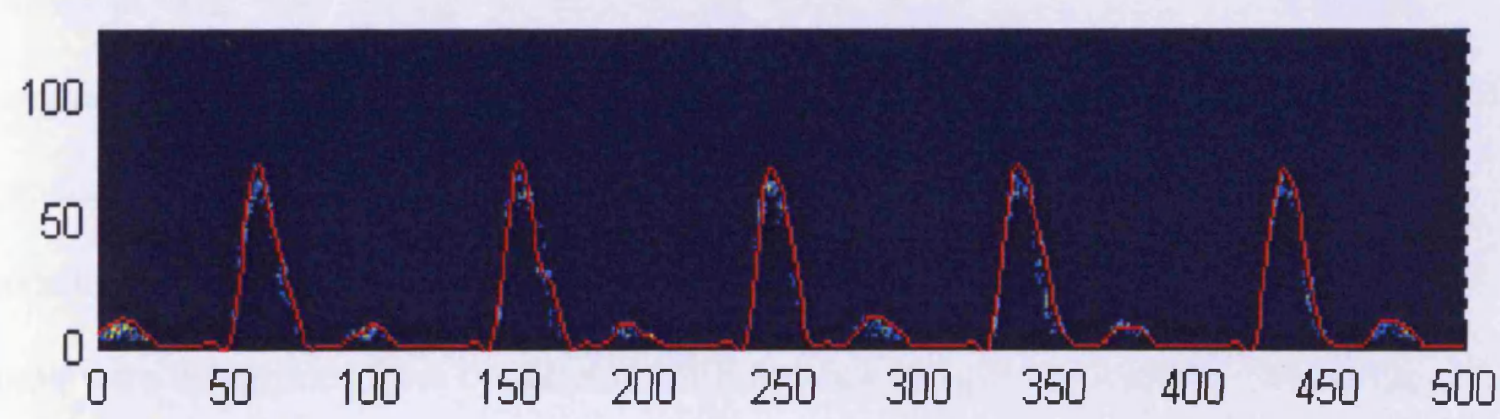

Figure 4.6b: MFE (indicated by red line) for a spectrogram containing a low level of background noise with $20 \%$ filter threshold applied 


\subsubsection{Automatic Thresholding}

An automatic thresholding technique was implemented in the MATLAB code to optimise the threshold level for filtering for each spectrogram individually, depending upon the level of low power noise present in the spectrogram background. This involved sampling the background noise from specific regions within the background outside the true arterial signal. The pulse foot markers indicating the start and end of each systolic peak were used to demarcate regions lying in between individual waveform cycles. This enabled the background noise to be sampled from the background regions lying in between each systolic peak, and ensured the higher amplitudes from the true arterial signal were not selected. The threshold for 'grass cutting' was then set depending upon the noise levels present within the sampled regions.

In this technique, the maximum power amplitude within each vertical column of the forward flow spectrogram matrix was determined and the mean value was calculated. The initial threshold level for 'grass cutting' was set to $55 \%$ of this mean level. This threshold was high enough to remove the background noise from spectrograms containing a high level of background noise, but still enabled reasonable MFEs to be produced from spectrograms containing low levels of background noise. The locations of the systolic peaks and the pulse foot at the start and end of each systolic peak were determined from the filtered MFE for each complete waveform cycle in the spectrogram, as described above. The location of the pulse foot at the start of the last incomplete pulse was also calculated. The pulse foot locations were used to select regions within the background in between each pulse, away from the true arterial 
signal. The height and vertical positions of the sample regions were set as a percentage of the systolic peak height. It was ensured that the vertical position of sample regions lay above the second forward flow phases. Examples of the sampled background regions are indicated in mid-blue on the spectrogram shown in Figure 4.7.

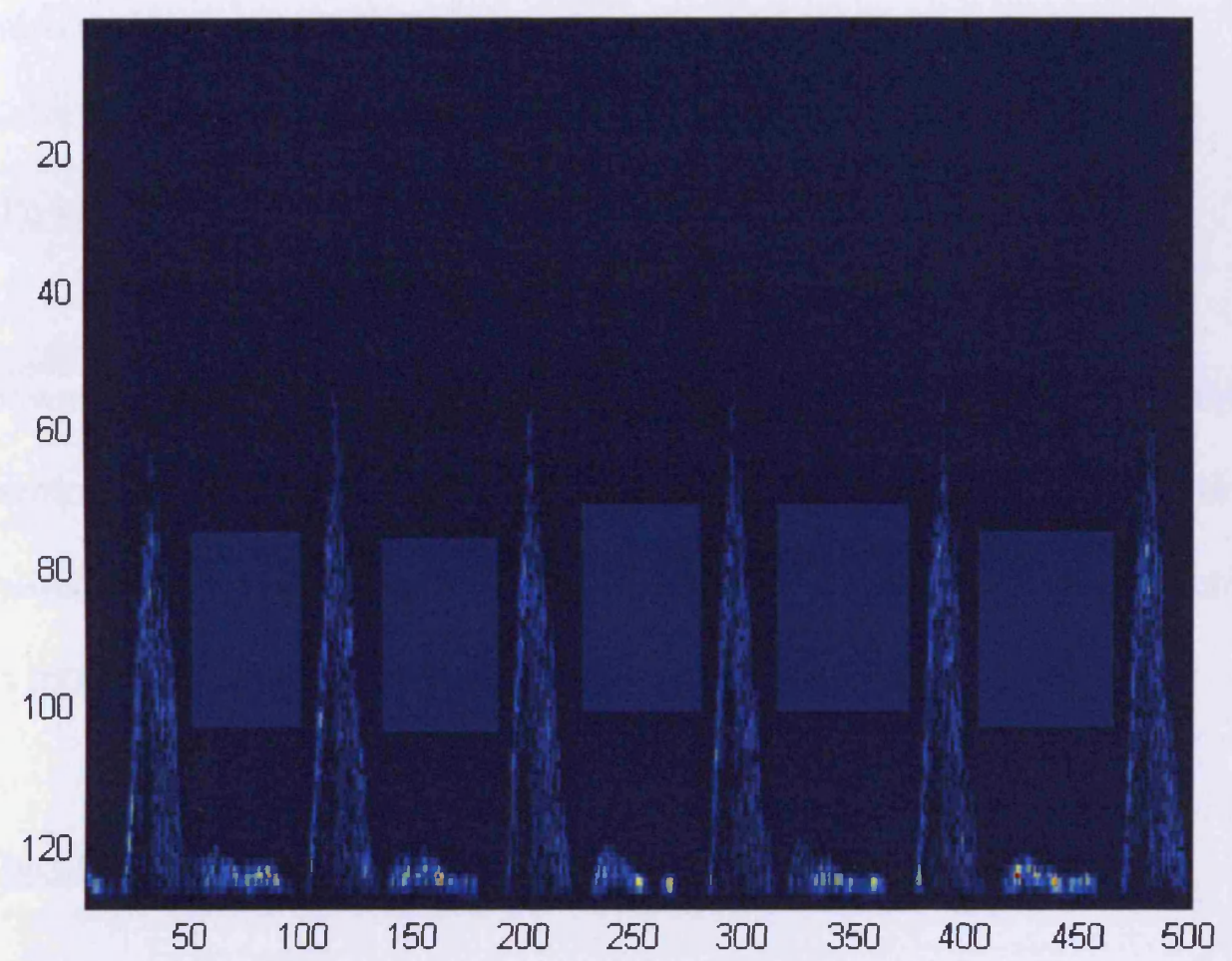

Figure 4.7: Forward flow CFA spectrogram with sampled regions indicated in mid-blue.

The maximum power levels within each of the sampled regions were calculated and the threshold level for 'grass cutting' was set to $120 \%$ of the maximum level. All pixels within the spectrogram matrix with an amplitude below this threshold level were set to zero. This method optimised the removal of background noise from the spectrograms, whilst maintaining a sufficient level of data in the CFA waveforms to permit further analysis. 
To ensure the method operated correctly for all the 60 AAA and 60 normal waveforms, the process was performed on each waveform individually to ensure the initial peak and pulse foot locations and the sample regions were correctly placed. After the automatic thresholding technique had been implemented, the forward flow maximum frequency envelope and peak and feet locations were recalculated and superimposed onto the CFA waveforms in the filtered spectrogram to ensure they were also positioned correctly.

\subsubsection{Power Level Normalisation}

The power (pixel amplitude) levels within the spectrogram were dependent upon the gain settings used in the waveform measurement. Therefore the power levels were normalised by dividing the power level contained in each pixel of the spectrogram matrix by the maximum power level within the spectrogram.

\section{$\underline{4.4 \text { Calculation of Waveform Parameters }}$}

Once automatic thresholding had been applied to the spectrogram and the power levels had been normalised, the matrix was manipulated in order to calculate a number of parameters from which the features noted in the visual inspections could be described. The maximum frequency envelopes were calculated to examine the contours of the waveforms, with the aim of capturing the spikes on the systolic down stroke. The minimum frequency envelopes were calculated with the aim of providing a measure to describe the level of spectral broadening. The power levels within the spectrum were used to calculate a 'Power Envelope' to describe the variation of the 
power levels within the waveform cycles. The location markers for the systolic, reverse and 2 nd forward flow phases were calculated and used to examine the parameters within the different flow phases of the waveform cycles.

\subsubsection{Maximum Frequency Envelope Calculation}

The MFEs for the forward and reverse flow components were calculated separately using the $95 \%$ MFE method. The positions of the forward and reverse flow MFEs for each of the 60 AAA and 60 normal waveforms were checked by plotting and superimposing the forward and reverse flow MFEs onto the MATLAB spectrogram. The forward and reverse flow MFEs were then normalised by dividing the amplitude of each point in the envelope by the maximum amplitude of the forward flow MFE.

\subsubsection{Minimum Frequency Envelope Calculation}

A technique similar to the maximum frequency envelope calculation was employed to calculate an envelope to follow the 'inner' profile of the forward flow waveform component. This 'minimum frequency envelope' was derived from the amplitude of the Doppler frequency spectrum by calculating the point of the $95 \%$ power level working down from the Maximum Frequency envelope. The amplitudes of the pixels in each column of the data matrix (corresponding to a point in time on the spectrogram) were summed from the level of the maximum frequency envelope down to the baseline (middle row of the spectrogram matrix). The minimum frequency point was calculated as the pixel that corresponded to $95 \%$ of the summed frequency amplitudes down from the MFE at this point in time. The positions of the minimum 
frequency envelopes were checked for each waveform by plotting and superimposing the minimum frequency envelope onto the forward flow MATLAB spectrogram. The minimum frequency envelopes were then normalised by dividing the amplitude of each point along the envelope by the maximum amplitude of the un-normalised forward flow maximum frequency envelope.

\subsubsection{Bandwidth Envelope Calculation}

To provide a measure of the level of spectral broadening in the forward flow, a 'bandwidth envelope' was calculated by subtracting the value of minimum frequency envelope from the value of the maximum frequency envelope at each point in time. An example of the bandwidth envelope is shown in Figure 4.8. The white line indicates the maximum frequency envelope, the yellow line indicates the minimum frequency envelope and the red line indicates the bandwidth envelope. The bandwidth envelopes were also normalised with respect to the maximum amplitude of the forward flow MFE.

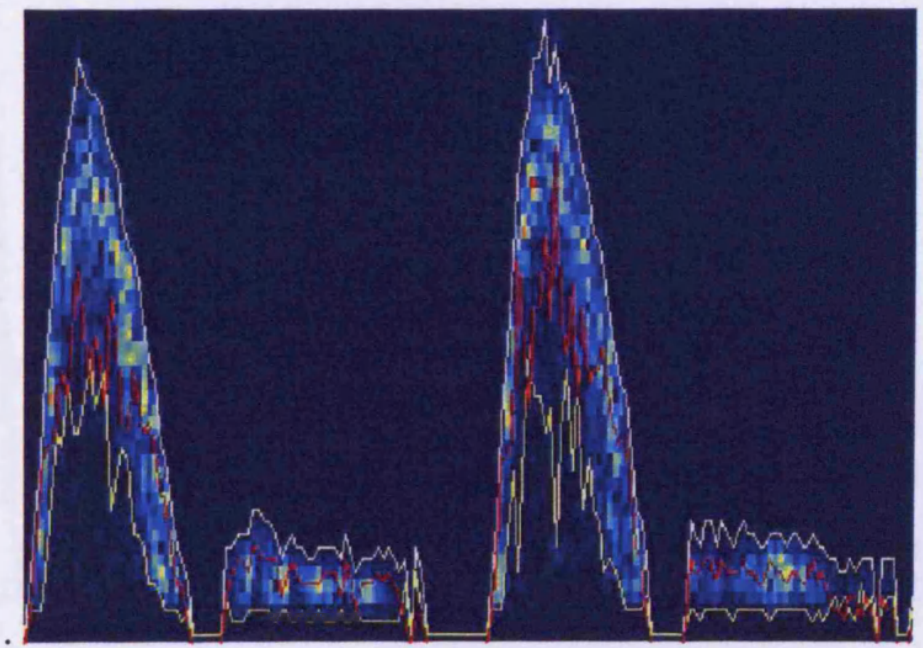

Figure 4.8: Example of forward flow spectrogram with maximum frequency envelope, (white line), minimum frequency envelope (yellow line), and bandwidth envelope (red line) superimposed. 


\subsubsection{Power Envelope Calculation}

The normalised power levels of the frequencies occurring at each point in time were summed to produce parameter called the 'total power envelope'. Separate total power envelopes were calculated for the forward and reverse flow components by summing the power levels of the pixels in each column from the baseline (middle row in the spectrogram) up to the top row of the spectrogram matrix, and from the baseline down to the bottom row of the spectrogram matrix respectively. Two more power envelopes were calculated by summing the power levels in each matrix column from the baseline up to the level of the Butterworth filtered MFEs in the forward and reverse directions. An example of a total power envelope for the forward flow component is plotted in Figure 4.9. The large peaks represent the power within the systolic peak regions and the smaller peaks represent the power within the second forward flow phases.

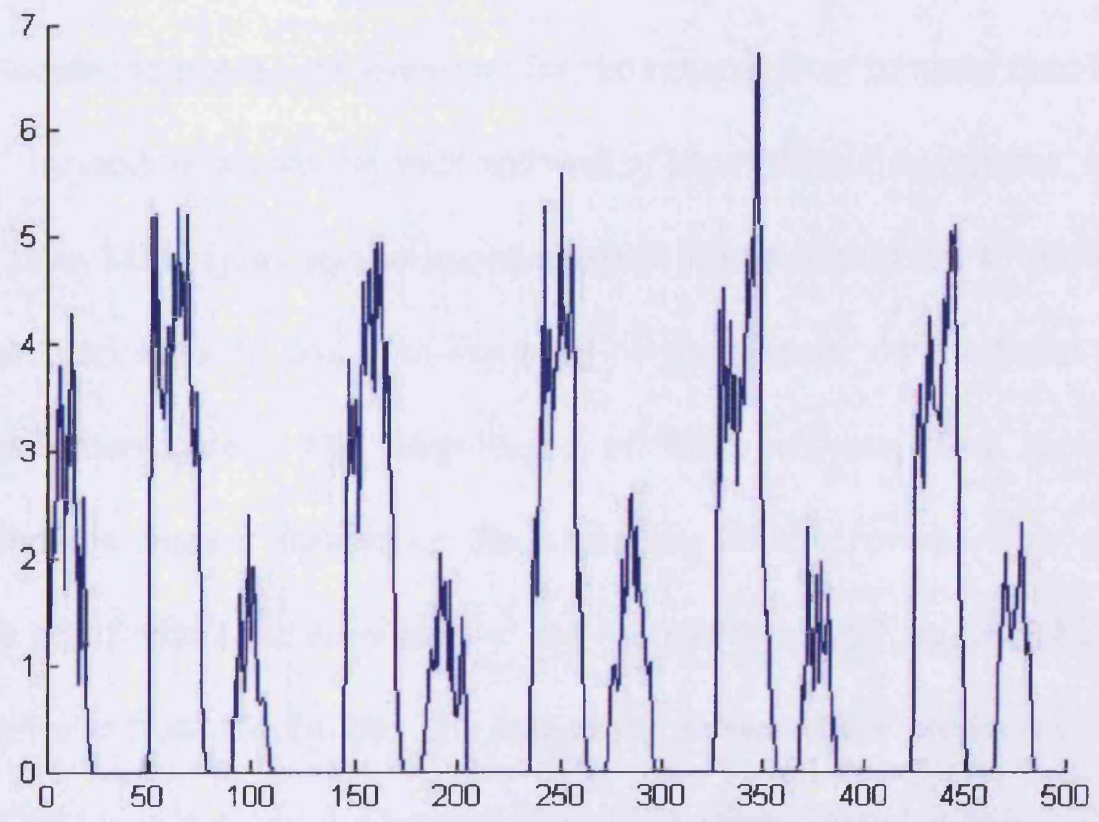

Figure 4.9: Example of Total Power Envelope. 


\subsubsection{Systolic Peak Markers}

The positive Butterworth filtered MFEs were interrogated using the method described in Section 4.3.2.1 to calculate the locations of the start of the systolic flow phase, the systolic peak and the end of the systolic flow phase. To check the locations of the calculated markers the MFEs were plotted with the peak markers superimposed. The systolic peak markers were also used to calculate the length of the systolic peak, the length of the systolic upstroke region and the length of the systolic down-stroke region for each waveform cycle. The 'beat lengths' between each of the waveform cycles were calculated as the difference between the pulse foot locations of subsequent systolic peaks.

\subsubsection{Reverse Flow and Second Forward Flow Phase Markers}

The shape of the reverse flow phases were often more complex than the systolic peaks and therefore application of the differential method to the reverse MFE did not adequately locate the pulse foot locations for the reverse flow in more than half of the waveforms. Instead, to locate the start and end of the reverse flow phases, sections of the reverse flow MFE lying in the region from 5 points (columns) to the left of the systolic peak marker to 40 points to the right of the systolic down-stroke pulse foot marker were interrogated. The amplitudes of these reverse flow sections were examined and the marker indicating the beginning of the reverse flow phase was placed at the point where the amplitude of the reverse flow MFE exceeded $25 \%$ of the maximum reverse flow amplitude. The end of the reverse flow phase was calculated by counting backwards along the reverse flow MFE from the pulse foot at the start of 
the subsequent systolic peak until the height of the revere flow MFE exceeded $10 \%$ of the maximum reverse height within the cycle.

The markers for the end of the second forward flow phases were located in a similar manner by counting backwards from the subsequent systolic pulse foot along the forward flow MFE. The location marker was positioned at the point at which the height of the MFE exceeded $30 \%$ of the maximum height of the MFE region lying between the end of the systolic peak of the waveform cycle and the pulse foot of the subsequent systolic peak.

The locations of the markers for the reverse flow and second forward flow phases were checked for each waveform by superimposing the location markers onto the spectrogram as shown in Figure 4.10.

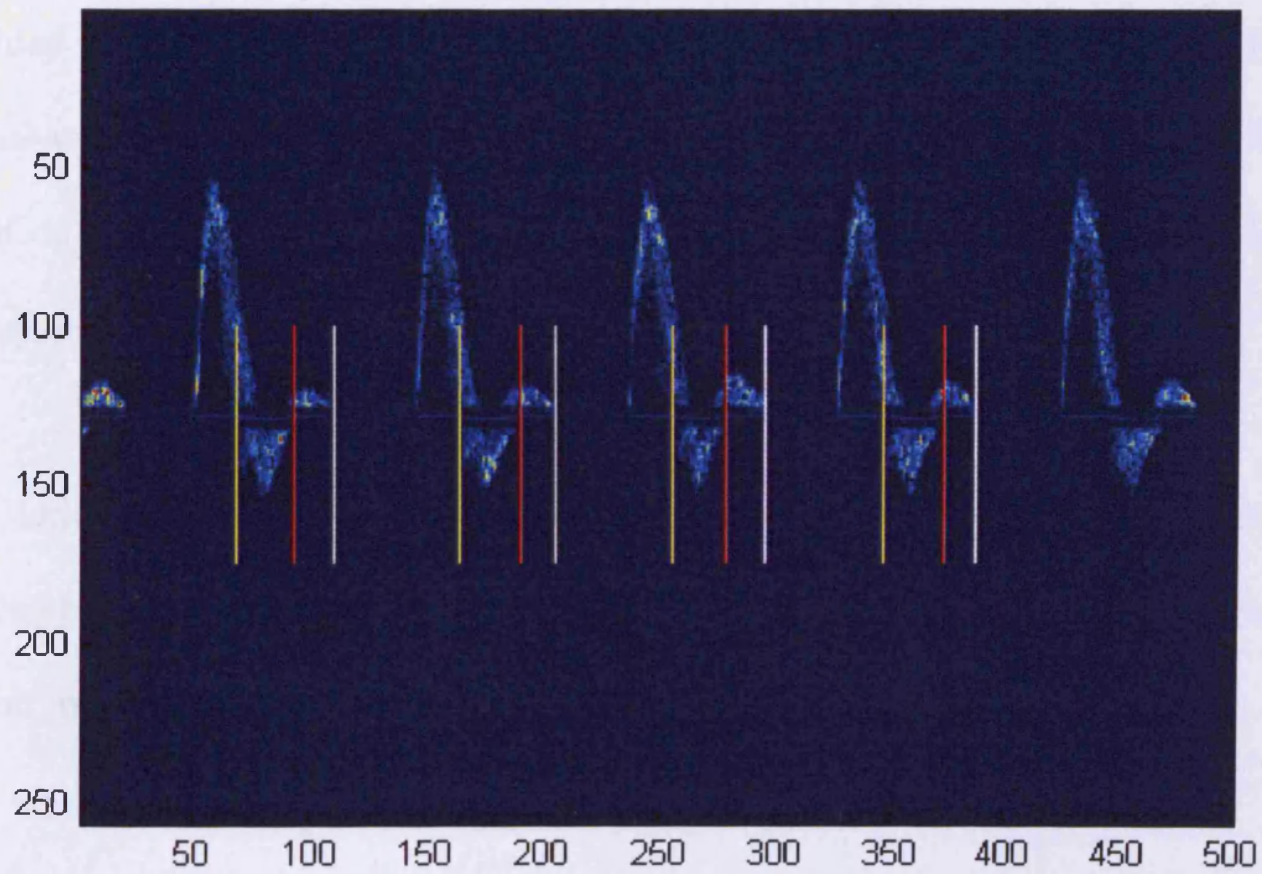

Figure 4.10: Markers indicating the calculated start and end of the reverse flow phase (yellow and red lines respectively) and the end of the $2^{\text {nd }}$ positive flow phase (white line). 


\subsection{Identification of Measures to Describe CFA Waveform Features}

The maximum frequency envelopes, bandwidth envelopes, power envelopes and the flow phase markers were used to calculate a number of different indices to provide measures of aspects of the 5 features noted visually: Spectral broadening on the systolic down-stroke, the appearance of spikes on the systolic down-stroke, an irregular reverse flow pattern, simultaneous forward and reverse flow, and elongation of the waveform during the cardiac cycle. Each of the indices were calculated for each individual waveform cycle in the spectrogram, except for the first and last waveform cycles which were excluded to ensure all cycles were complete. The mean index value per waveform cycle was then calculated.

Each index was calculated and plotted for the left and right CFA waveforms for the 30 AAA and 30 normal patients in the no significant femoro-popliteal atherosclerotic disease groups. The plots were visually inspected to determine whether the index provided any discrimination between the AAA and normal waveforms. For the indices which showed a level of discrimination, a number of threshold levels were tested on the plots to determine the optimum level to best separate the AAA and normal waveforms.

Each index provided a measure for one of the visual features, and in effect, the threshold levels were used to determine objectively whether or not the feature was present in a waveform. 


\subsubsection{Spectral Broadening Indices}

A number of different indices to describe the first feature noted in the visual analysis of increased spectral broadening on the systolic down-stroke, illustrated in Figure 4.11 were generated by examining the bandwidth and power envelope distributions in the systolic peak regions. The location markers were used to identify the systolic peak, systolic upstroke, and systolic down-stroke regions for each complete waveform cycle within the spectrogram.

a)

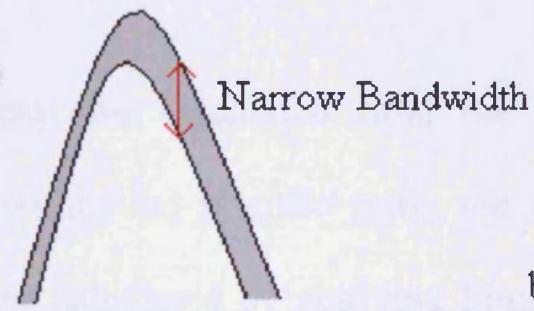

b)

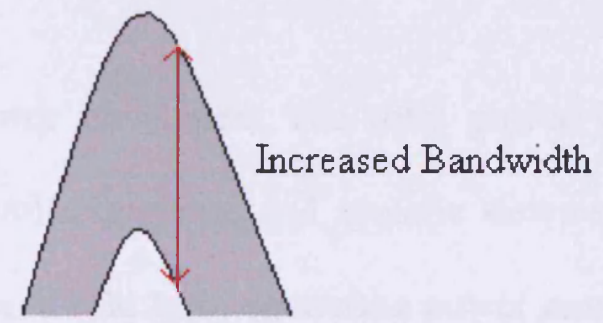

Figure 4.11: Illustration of systolic peaks showing a) normal systolic down-stroke bandwidth and b) increased systolic down-stroke bandwidth with AAA

The bandwidth envelopes were interrogated to determine the maximum bandwidth levels within the systolic peak, systolic upstroke and systolic down-stroke regions for each complete waveform cycle and the mean values per cycle were calculated. The difference and the ratio between the maximum bandwidth level on the systolic downstroke and the maximum bandwidth level on the systolic upstroke were also calculated. 
The bandwidth envelopes for the systolic peak and the systolic upstroke and downstroke regions (identified using the flow phase markers) were integrated for each complete waveform cycle by the application of Simpson's rule. The lengths of each of the systolic peaks, systolic upstroke and systolic down-stroke regions were calculated from the phase location markers. The mean bandwidth level in each region was calculated by dividing the bandwidth integral by the length of the corresponding region. The mean bandwidths for the systolic peak, the systolic upstroke and systolic down-stroke regions were calculated for each complete waveform cycle and the mean values per cycle were calculated. The difference and the ratio between the mean bandwidth over the systolic down-stroke region and the mean bandwidth over the systolic upstroke region were also calculated.

Indices were also calculated from the power envelopes. The total power levels contained within the systolic peak, the systolic upstroke and systolic down-stroke regions were calculated by applying Simpson's rule to integrate the power envelope within these regions. The difference and the ratio between the total power level in the systolic down-stroke and the total power level in the systolic upstroke regions were also calculated. Another index was calculated by dividing the total power within the systolic down-stroke by the bandwidth integral of the systolic down-stroke.

The above indices were calculated for each individual, complete waveform cycle within each spectrogram and the mean values per cycle were calculated. The indices were plotted for the left and right CFA waveforms from the 30 AAA and 30 normal patients. An explanation of the format of the index plots is given in Appendix 5. The plots were visually examined to determine which of the indices provided 
discrimination between the two patient groups. A narrow bandwidth indicates how 'laminar' the blood flow is. In theory in the presence of an AAA the flow may be more disturbed and therefore in general the bandwidth may be wider. Two of the above indices to provide measures to describe the level of spectral broadening: the maximum systolic down-stroke bandwidth and the maximum upstroke bandwidth, showed a level of discrimination between the AAA and normal waveforms.

The maximum systolic down-stroke bandwidth index is plotted for the right and left CFA waveforms from the 30 AAA and 30 normal patients in Figure 4.12. 


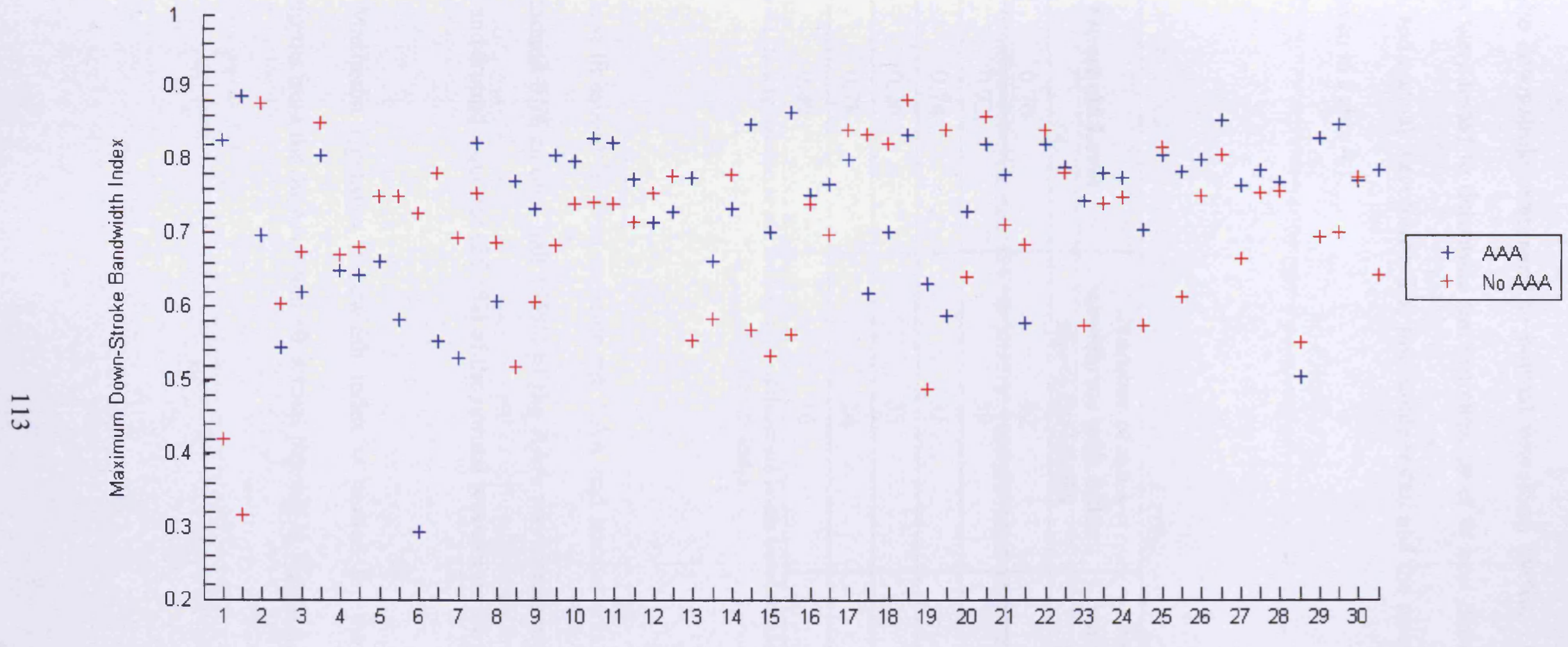

Patient (Left and Right CFA Waveform)

Figure 4.12: Maximum Down-stroke Bandwidth Index plotted for the left and right CFA waveforms from the $30 \mathrm{AAA}$ and 30 normal patients without significant atherosclerotic arterial disease 
In general, the AAA waveform cycles have a higher maximum bandwidth level on the systolic down-stroke compared to normal waveform cycles. A range of threshold levels were tested to determine the optimum level to best discriminate between the AAA and normal waveforms. The thresholds tested and the results for each threshold are given in Table 4.1.

\begin{tabular}{|c|c|c|}
\hline Threshold Level & $\begin{array}{c}\text { Number of AAA } \\
\text { waveforms with index } \\
\text { above threshold }\end{array}$ & $\begin{array}{c}\text { Number of normal } \\
\text { waveforms with index } \\
\text { above threshold }\end{array}$ \\
\hline 0.70 & 42 & 40 \\
\hline 0.72 & 39 & 30 \\
\hline 0.74 & 35 & 24 \\
\hline 0.76 & 33 & 16 \\
\hline 0.78 & 24 & 12 \\
\hline 0.80 & 16 & 11 \\
\hline
\end{tabular}

Table 4.1: Discrimination levels for the range of threshold levels tested for the Maximum Down-stroke Bandwidth Index.

The best threshold level to separate the AAA and normal waveforms by this index was around 0.76 as over half (55\%) of the AAA waveforms are above the threshold level and around a quarter (26.6\%) of the normal waveforms are above the threshold.

The Maximum Upstroke Bandwidth index is plotted for the left and right CFA waveforms from the $30 \mathrm{AAA}$ and 30 normal patients in Figure 4.13. 


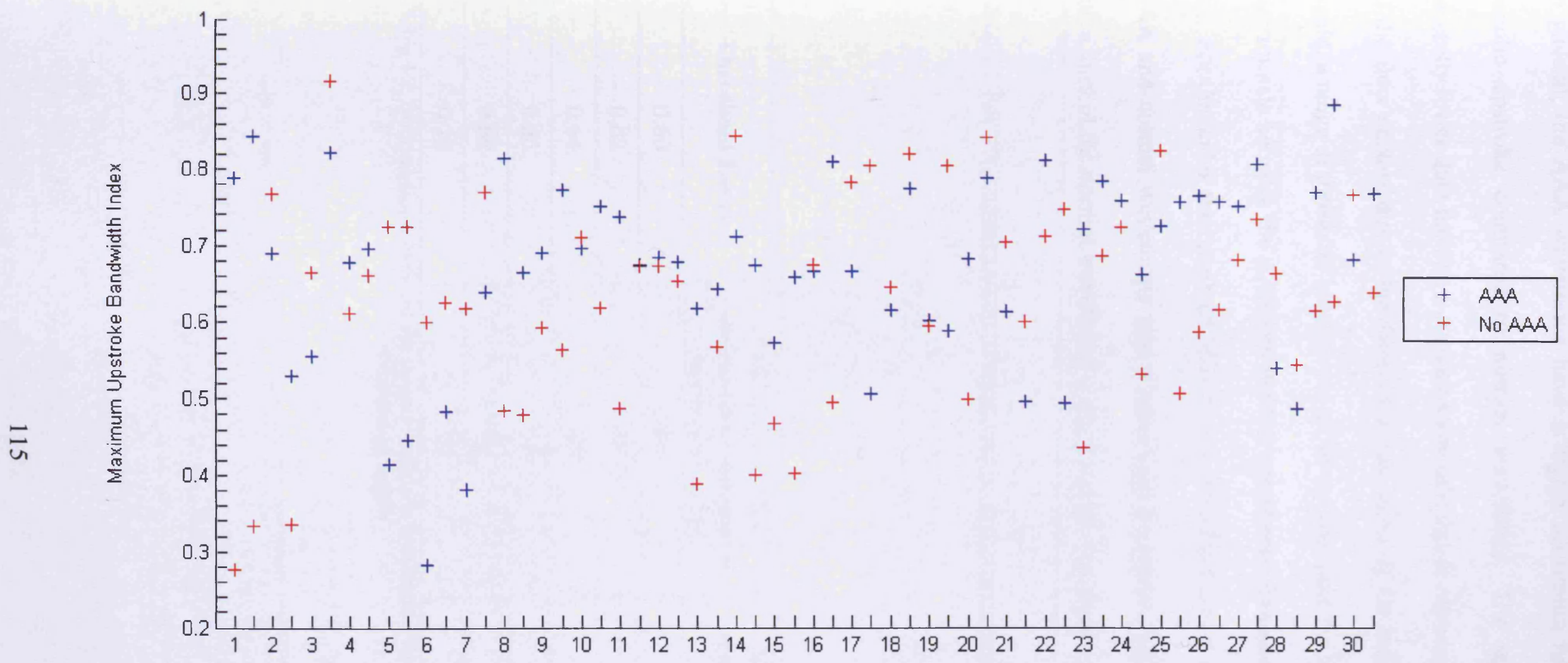

Patient (Left and Right CFA Waveform) atherosclerotic arterial disease 
In general, the AAA waveforms have a higher maximum bandwidth level on the systolic upstroke compared to normal waveforms. The upstroke bandwidth is generally lower than the down-stroke bandwidth; this is expected due to the alignment of the flow vectors due to the forward acceleration of the bulk flow during systole. Again, a range of threshold levels were tested to determine the optimum index level to discriminate between the AAA and normal waveforms. The results are given in Table 4.2. For this index the threshold level which provided the best separation between the AAA and normal waveforms was around 0.68 for which half the AAA waveforms and a third of the normal waveforms were above the threshold.

\begin{tabular}{|c|c|c|}
\hline Threshold Level & $\begin{array}{c}\text { Number of AAA } \\
\text { waveforms with index } \\
\text { above threshold }\end{array}$ & $\begin{array}{c}\text { Number of normal } \\
\text { waveforms with index } \\
\text { above threshold }\end{array}$ \\
\hline 0.60 & 46 & 37 \\
\hline 0.62 & 42 & 32 \\
\hline 0.64 & 42 & 29 \\
\hline 0.66 & 39 & 27 \\
\hline 0.68 & 30 & 20 \\
\hline 0.70 & 24 & 19 \\
\hline
\end{tabular}

Table 4.2: Discrimination levels for the range of threshold levels tested for the Maximum Upstroke Bandwidth Index. 


\subsubsection{Systolic Down-Stroke ‘Spikes' Index}

To second feature noted in the visual analysis was the presence of 'spikes' on the down-stroke of the systolic peak, illustrated in Figure 4.14.
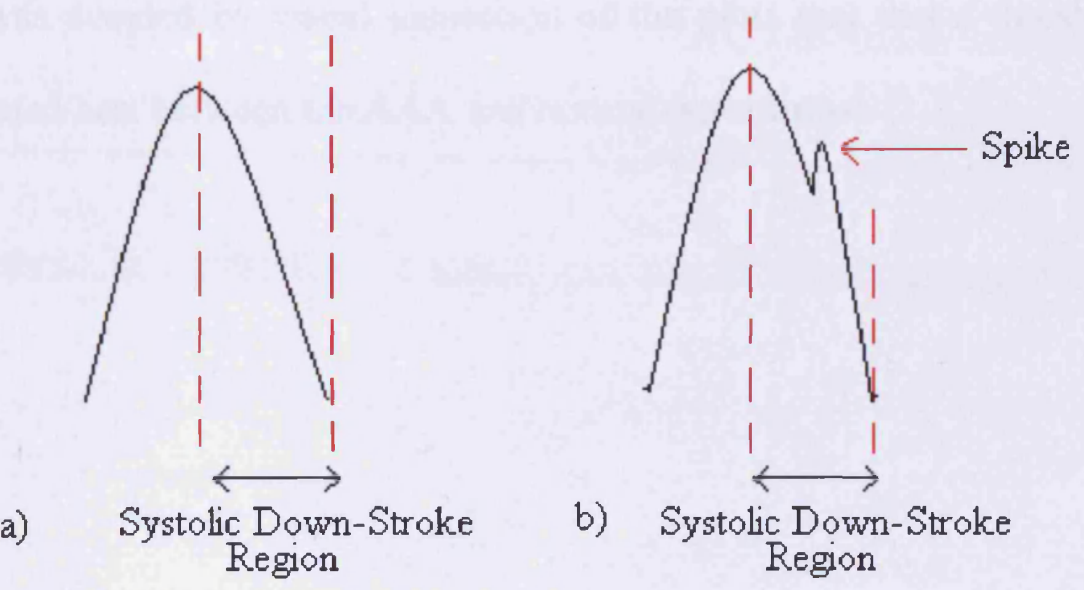

Figure 4.14: Illustration of systolic peaks showing a) normal systolic down-stroke and b) systolic down-stroke with spike

In order to automatically detect this feature MATLAB code was written to identify discontinuities on the down-stroke. This was achieved by examination of the differentials between subsequent points of the MFE in the systolic down-stroke region. The method 'counted' along the MFE of the systolic down-stroke region until the amplitude of the subsequent point was greater than the last. A 'spike' was identified if the deflection continued for $>2$ further subsequent points along the MFE. This criterion was set to try and discount irregularities in the profile occurring due to noise. The process terminated at the pulse foot at the end of the systolic peak. The 
number of 'spikes' in the systolic down-stroke region of every complete waveform cycle in the spectrogram were calculated and then the mean number of spikes per waveform cycle was calculated.

The results for the right and left CFA waveforms from the 30 AAA and 30 normal patients for are plotted in Figures 4.15a and 4.15b respectively. On visual inspection of the AAA waveforms the spikes were not always present on every single systolic peak. It was decided by visual inspection of the plots that that a threshold of $\geq 0.5$ discriminated best between the AAA and normal waveforms. 


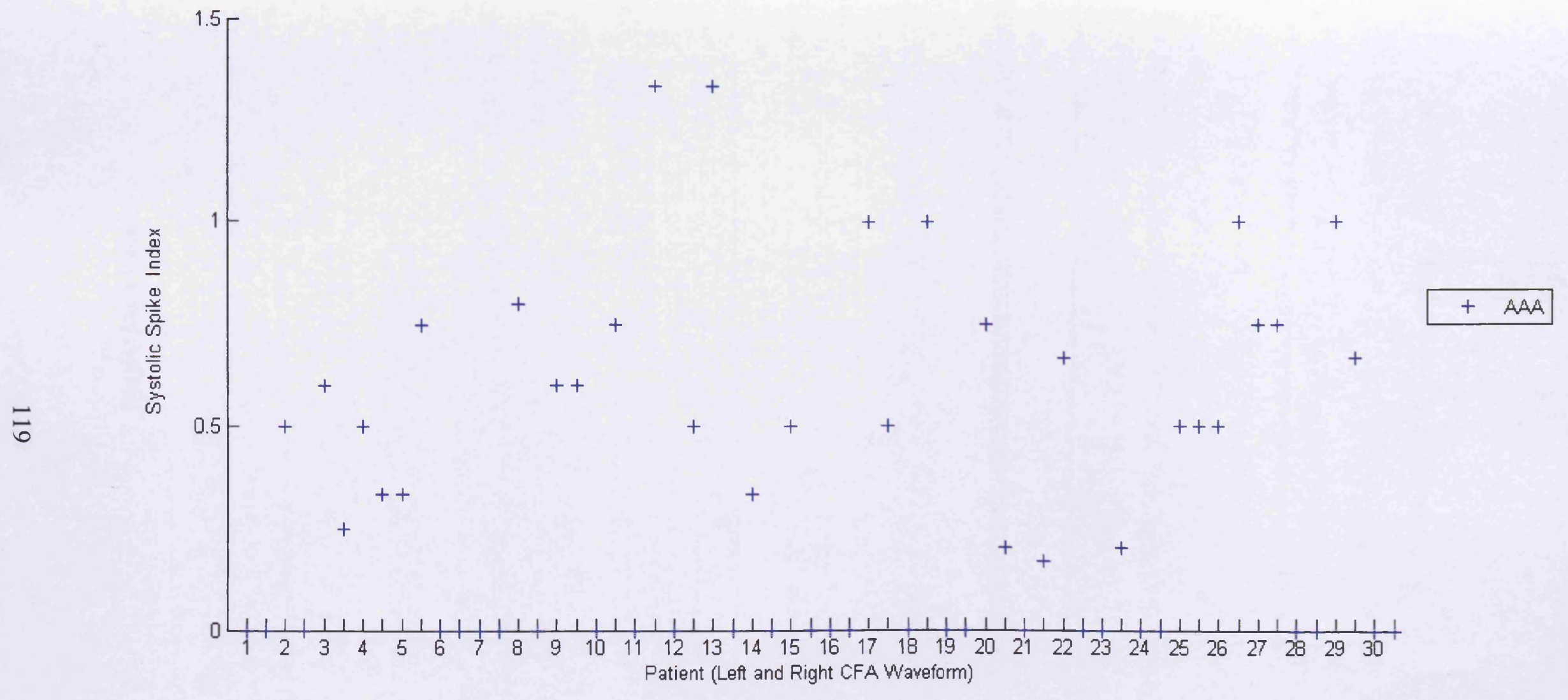

Figure 4.15a: Systolic Spike Index plotted for the left and right CFA waveforms for the 30 patients with AAA without significant atherosclerotic arterial disease 


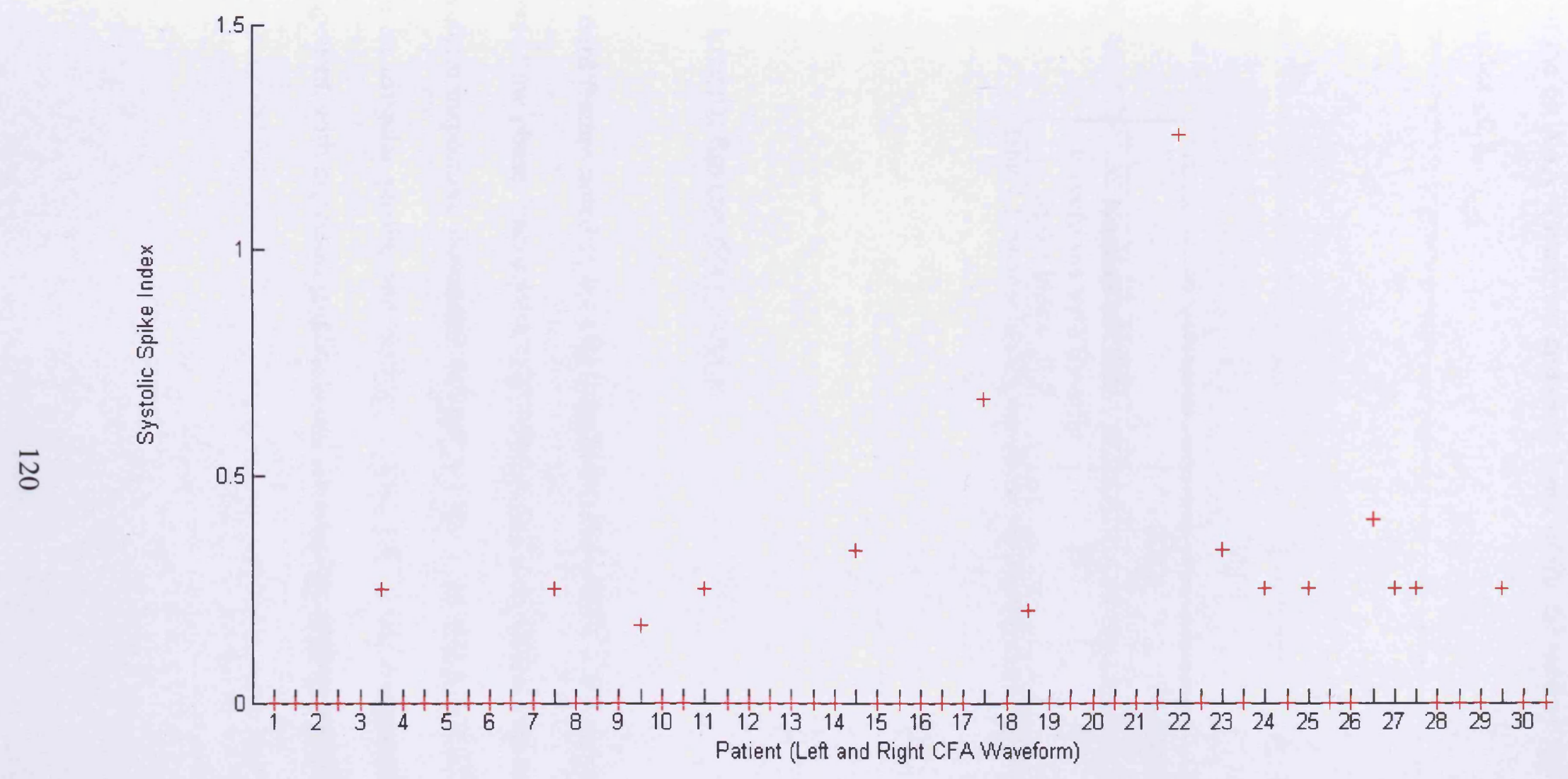

No AAA

Figure 4.15b: Systolic Spike Index plotted for the left and right CFA waveforms for the 30 patients without AAA and without significant atherosclerotic arterial disease 
The number of AAA and normal waveforms with a systolic spike index above the threshold level of $\geq 0.5$ are given in Table 4.3. This index and threshold level provided a very good level of discrimination between the AAA and normal waveforms, with 25 out of the 60 AAA waveforms and only 2 out of the 60 normal waveforms having a spike index $\geq 0.5$.

\begin{tabular}{|c|c|c|}
\hline & AAA & Normal \\
\hline $\begin{array}{c}\text { Number of CFA } \\
\text { waveforms with Systolic } \\
\text { Spike Index } \geq 0.5\end{array}$ & 25 & 2 \\
\hline
\end{tabular}

\subsubsection{Irregular Reverse Flow Indices}

The third feature noted in the visual analysis of the CFA waveforms was an irregular reverse flow phase. There were two different types of irregularity noted in the visual waveform inspection, illustrated in Figure 4.16: 1) an elongation of the reverse flow with an irregular profile and distinct spikes, and 2) an 'exaggerated' reverse flow component, with increased amplitude and an irregular, slightly square profile. 

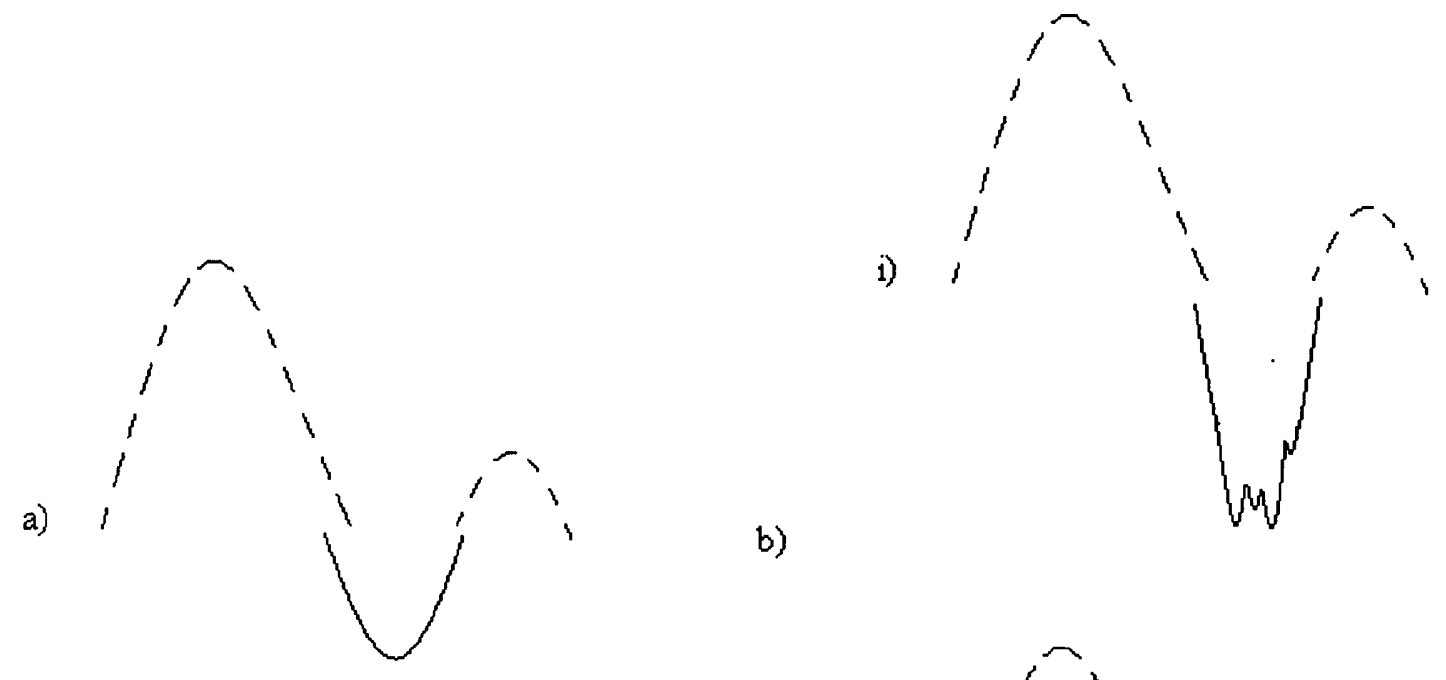

Figure 4.16: Ilustration of a) normal reverse flow patten, $b$ i) reverse flow component with increased amplitude and an irregular, square profile, and $b$ ii) elongation of the reverse flow component with irregular profile

A method similar to that used to identify the spikes on the systolic down-stroke was applied to the reverse flow Butterworth filtered maximum frequency envelope to detect fluctuations in the profile of the reverse flow phase. The criteria for the length of the deflection in the differentials was increased to $\geq 3$ and a second criteria was set so that the height of the deflection had to exceed $30 \%$ of the maximum reverse flow height to be counted as a 'fluctuation'. A number of different regions in the reverse flow were tested. The best results were achieved for the reverse flow region lying under the systolic down-stroke from the systolic peak to the pulse foot of the systolic down-stroke plus half the systolic peak length. This region incorporated the irregularities in the reverse flow arising from the simultaneous forward and reverse 
flow in the systolic down-stroke as well as those occurring in the true reverse flow phase. As for the systolic spikes, the number of 'fluctuations' were counted in the reverse flow region of every complete waveform cycle in the spectrogram and the mean number of fluctuations per waveform cycle was calculated.

The number of fluctuations for the right and left CFA waveforms from the 30 AAA and 30 normal patients are plotted in Figures $4.17 \mathrm{a}$ and $4.17 \mathrm{~b}$ respectively. A number of threshold levels were tested and the threshold of $\geq 2$ fluctuations provided the best separation between the AAA and normal waveforms. The number of AAA and normal waveforms with a reverse flow fluctuation index above the threshold level of $\geq 2$ are given in Table 4.4. Again, this index and threshold level provided a very good level of discrimination between the AAA and normal waveforms, with 27 out of 60 AAA waveforms and only 8 out of the 60 normal waveforms above the threshold. 


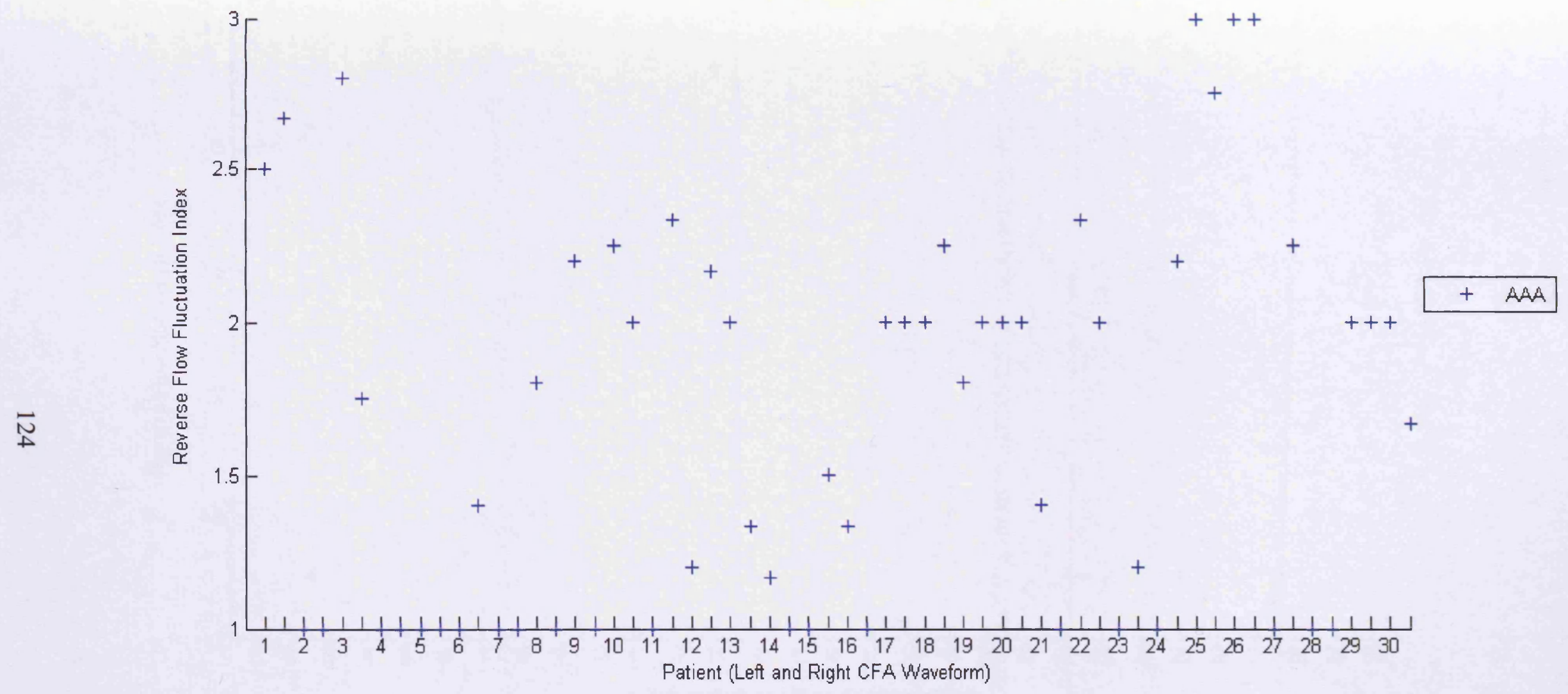

Figure 4.17a: Reverse Flow Fluctuation Index plotted for the left and right CFA waveforms for the 30 patients with AAA without significant atherosclerotic arterial disease 


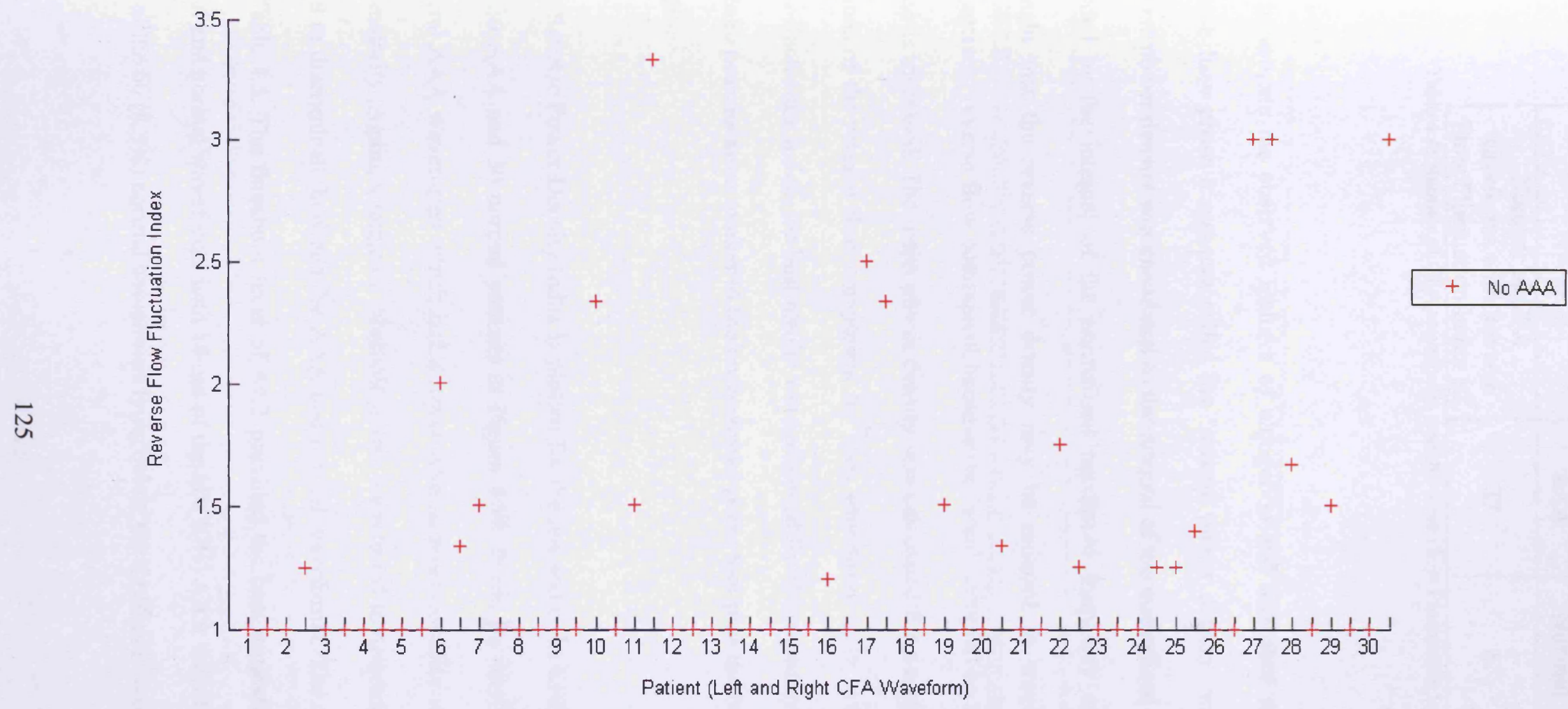

Figure 4.17b: Reverse Flow Fluctuation Index plotted for the left and right CFA waveforms for the 30 patients without AAA without significant atherosclerotic arterial disease 


\begin{tabular}{|c|c|c|}
\hline & AAA & Normal \\
\hline $\begin{array}{c}\text { Number of CFA } \\
\text { waveforms with Reverse } \\
\text { Flow Fluctuation Index } \geq 2\end{array}$ & 27 & 8 \\
\hline
\end{tabular}

Table 4.4: Number of CFA waveforms with Reverse Flow Fluctuation Index $\geq 2$

To investigate the observed features of elongation and increased amplitude of the reverse flow phase a measure called the 'reverse power density' was derived. The reverse power density was calculated as the integral of the normalised power envelope divided by the integral of the normalised maximum frequency envelope. It was thought that the reverse power density may be reduced in waveforms with an exaggerated reverse flow component because the 'area' occupied by the reverse flow phase is increased. The mean power density was calculated for a number of different regions of the reverse flow component for each waveform cycle. As for the reverse flow fluctuation index, the best results were achieved for the region lying between the systolic peak location marker to the down-stroke pulse foot plus the half systolic peak length.

The Reverse Power Density Index is plotted for the left and right CFA waveforms for the $30 \mathrm{AAA}$ and 30 normal patients in Figure 4.18. It can be seen that there were several AAA waveforms which had a lower reverse power density in comparison to the majority. Again, a range of threshold levels were tested to determine the optimum level to discriminate between the AAA and normal waveforms. The results are given in Table 4.5. The threshold level of $<4.2$ provided the best separation between the AAA and normal waveforms with 18 out of the 60 (30\%) AAA waveforms and only 5 out of the $60(8.3 \%)$ normal waveforms lying below the threshold level. 


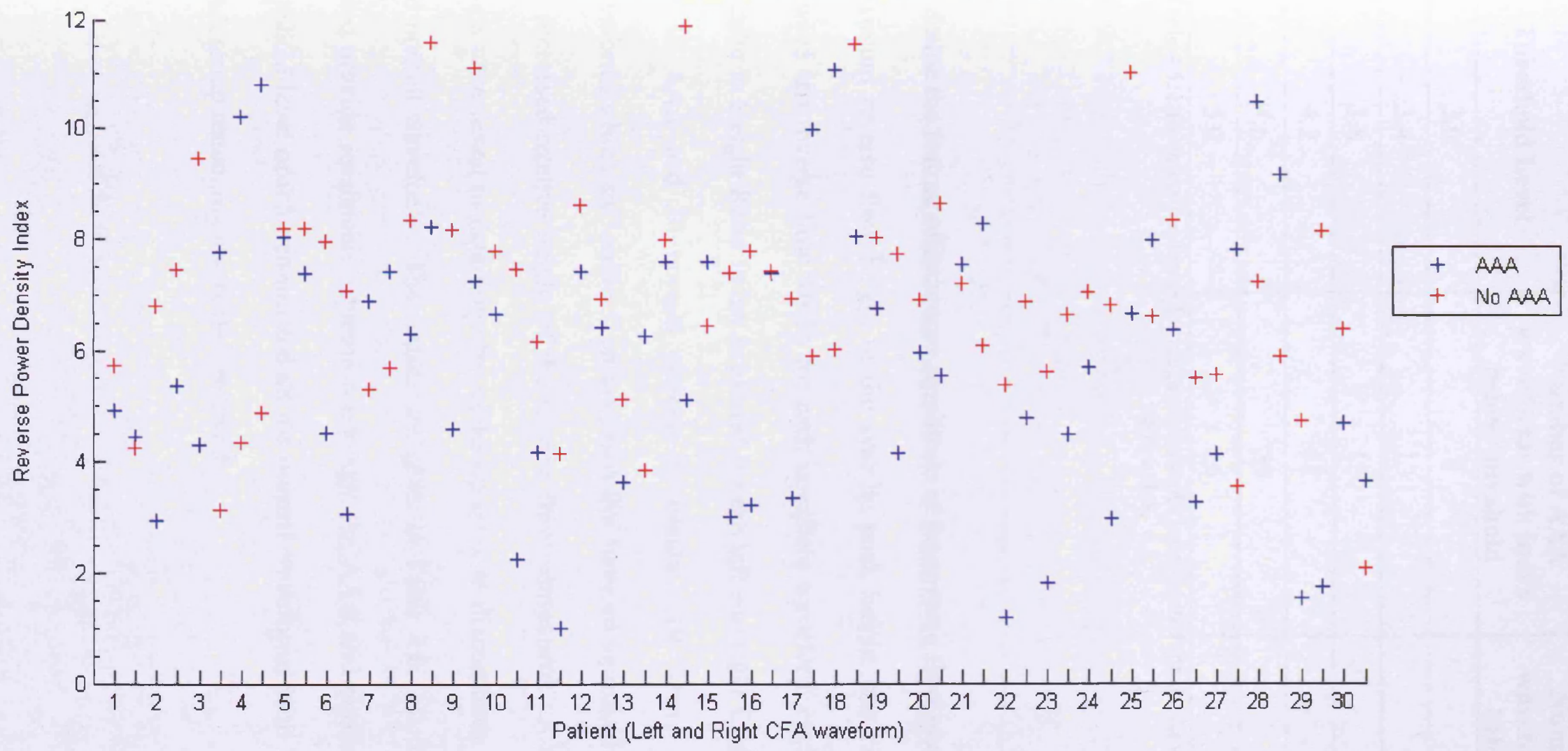

Figure 4.18: Reverse Power Density Index plotted for the left and right CFA waveforms from the $30 \mathrm{AAA}$ and 30 normal patients without significant atherosclerotic arterial disease 


\begin{tabular}{|c|c|c|}
\hline Threshold Level & $\begin{array}{c}\text { Number of AAA } \\
\text { waveforms with index } \\
\text { below threshold }\end{array}$ & $\begin{array}{c}\text { Number of normal } \\
\text { waveforms with index } \\
\text { below threshold }\end{array}$ \\
\hline 3.0 & 8 & 1 \\
\hline 3.4 & 13 & 2 \\
\hline 3.8 & 15 & 3 \\
\hline 4.2 & 18 & 5 \\
\hline 4.6 & 23 & 7 \\
\hline 5.0 & 26 & 9 \\
\hline
\end{tabular}

Table 4.5: Discrimination levels for range of threshold levels tested for the Reverse Power Density ratio index.

To define the feature of increased amplitude in the reverse flow phase, the ratio of the maximum reverse flow height to the systolic peak height was calculated from the forward and reverse flow MFEs for each complete waveform cycle. The Reverse to Systolic to Height Ratio Index is plotted for the left and right CFA waveforms from the 30 AAA and 30 normal patients in Figure 4.19. There are several AAA waveforms which are 'outliers' on the graph that have an increased height ratio due to the increased relative height of the reverse flow component. A range of threshold levels were tested to determine the optimum level to discriminate between the AAA and normal waveforms. The results are given in Table 4.6. All the threshold levels tested provide reasonable separation between the AAA and normal waveforms. The threshold level of 0.52 eliminated all the normal waveforms with 7 outliers from the AAA group remaining above the threshold. 


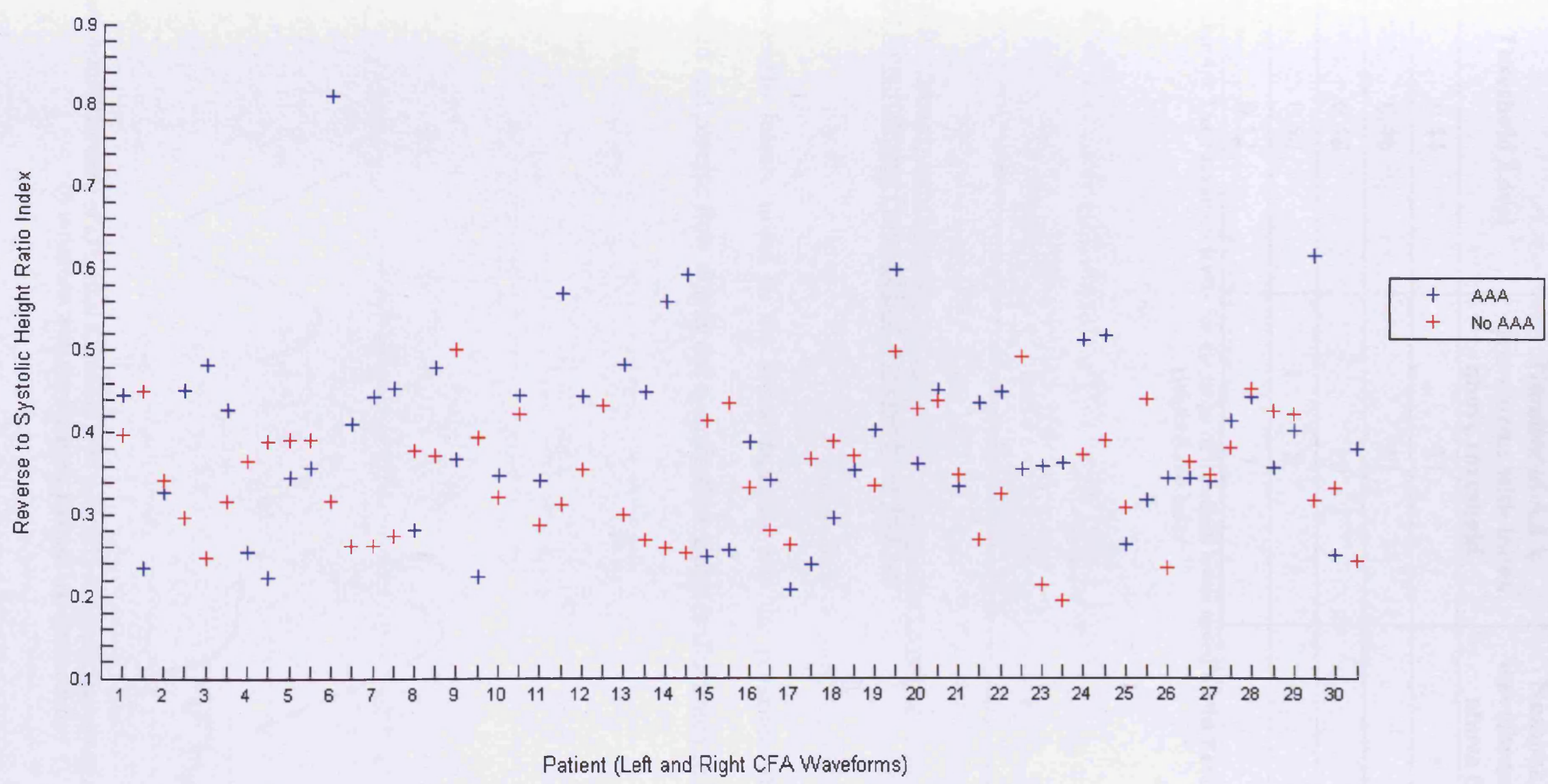

Figure 4.19: Reverse to Systolic Height Ratio Index plotted for the left and right CFA waveforms from the 30 AAA and 30 normal patients without significant atherosclerotic arterial disease 


\begin{tabular}{|c|c|c|}
\hline Threshold Level & $\begin{array}{c}\text { Number of AAA } \\
\text { waveforms with index } \\
\text { above threshold }\end{array}$ & $\begin{array}{c}\text { Number of normal } \\
\text { waveforms with index } \\
\text { above threshold }\end{array}$ \\
\hline 0.44 & 21 & 7 \\
\hline 0.46 & 11 & 3 \\
\hline 0.48 & 10 & 3 \\
\hline 0.50 & 8 & 2 \\
\hline 0.52 & 7 & 0 \\
\hline
\end{tabular}

Table 4.6: Discrimination levels for the range of threshold levels tested for the Reverse to Systolic Height Ratio Index.

\subsubsection{Simultaneous Forward and Reverse Flow Indices}

The fourth feature noted in the visual analysis was the presence of simultaneous forward and reverse flow during the systolic flow phase is illustrated in Figure 4.20.

a) 1

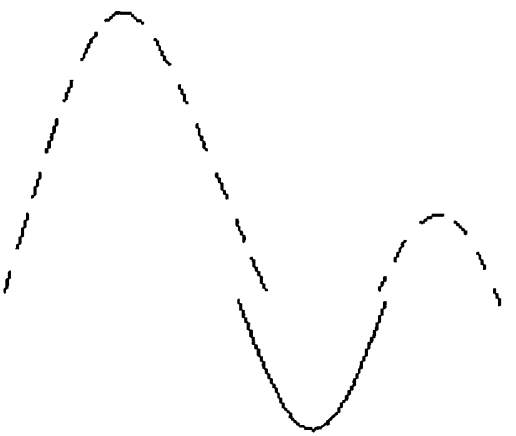

b)

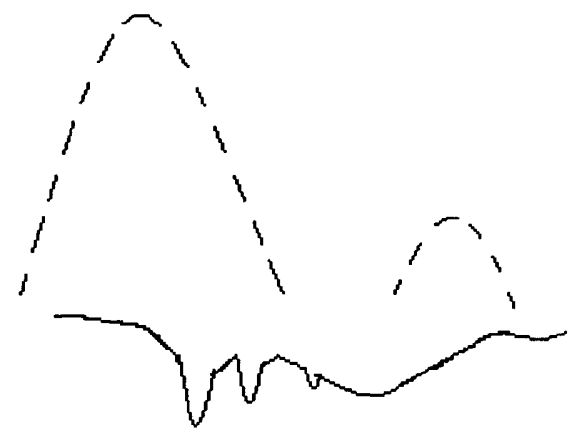

Figure 4.20: Illustration of a) normal waveform with minimal overlap of forward and reverse flow, and b) waveform with simultaneous forward and reverse flow 
Several indices were generated to provide a measure of the overlap in the forward and reverse flow phases under the systolic peak. The first index was generated to measure the time-delay between the systolic peak and the on-set of reverse flow. This was calculated by subtracting the position of the marker indicating the start of the reverse flow phase from the systolic peak marker position. The time-delay for each complete waveform cycle was normalised by dividing by the beat length. The time-delay index is plotted for the left and right CFA waveforms for the 30 AAA and 30 normal patients in Figure 4.21. There are several waveforms from AAA patients with a shorter time-delay between the systolic peak and the on-set of reverse flow. In 7 AAA waveforms the index value was negative indicating that a significant level of reverse flow occurred before the systolic peak was reached in the forward flow phase. The results for the threshold levels that were tested on this index to determine the best level to discriminate the between the AAA and normal waveforms are given in Table 4.7. The threshold level of $<0.04$ provides reasonable separation between the groups. 


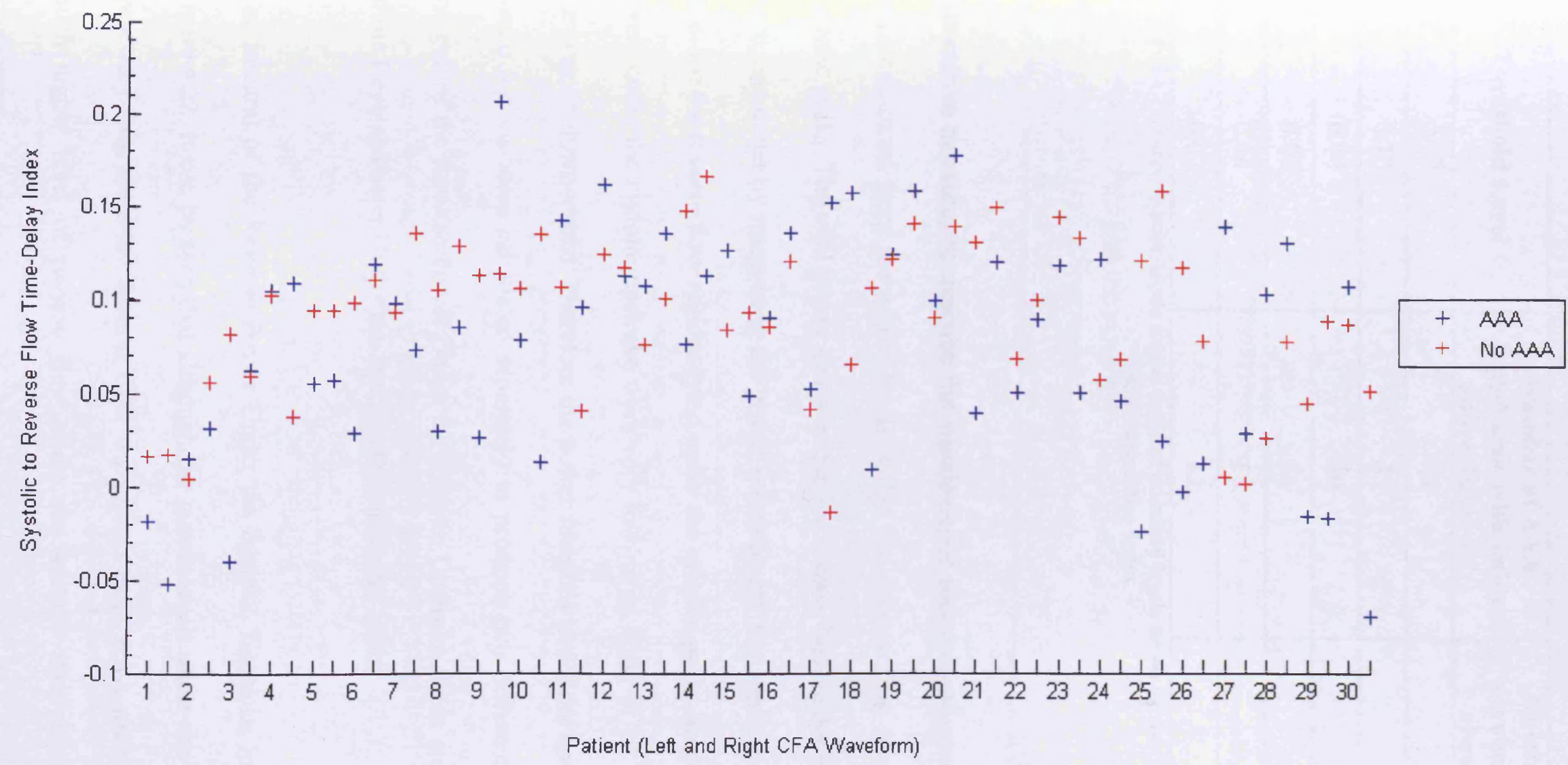

Figure 4.21: Systolic to Reverse Flow Time-Delay Index plotted for the left and right CFA waveforms from the 30 AAA and 30 normal patients without significant atherosclerotic arterial disease 


\begin{tabular}{|c|c|c|}
\hline Threshold Level & $\begin{array}{c}\text { Number of AAA } \\
\text { waveforms with index } \\
\text { above threshold }\end{array}$ & $\begin{array}{c}\text { Number of normal } \\
\text { waveforms with index } \\
\text { above threshold }\end{array}$ \\
\hline 0.12 & 44 & 40 \\
\hline 0.10 & 35 & 32 \\
\hline 0.08 & 30 & 21 \\
\hline 0.06 & 26 & 15 \\
\hline 0.04 & 18 & 8 \\
\hline 0.02 & 12 & 6 \\
\hline
\end{tabular}

Table 4.7: Discrimination levels for the range of threshold levels tested for the Systolic to Reverse Flow Time-Delay Index

Alternative measures to describe the simultaneous forward and reverse flow feature were calculated from the power levels within the reverse flow occurring under the systolic peaks. The total power levels within the reverse flow under each systolic peak were calculated by integrating the power envelope (by the application on Simpson's rule) for the reverse flow regions lying under the systolic peaks. In general, the power levels under the systolic upstroke were very low, to an order of 10 lower than under the systolic down-stroke. Therefore the power integrals under the upstroke and downstroke regions were calculated separately to produce two different indices: 1) The Integral of the Reverse Power Under the Systolic Upstroke Index and 2) The Integral of the Reverse Power Under the Systolic Down-Stroke Index.

The Integral of the Reverse Power Under the Systolic Upstroke Index is plotted in Figure 4.22. It can be seen that although the power levels under the systolic upstroke were very low, there are several CFA waveforms from AAA patients which have a much higher level of reverse flow under the systolic upstroke than the normal waveforms. 


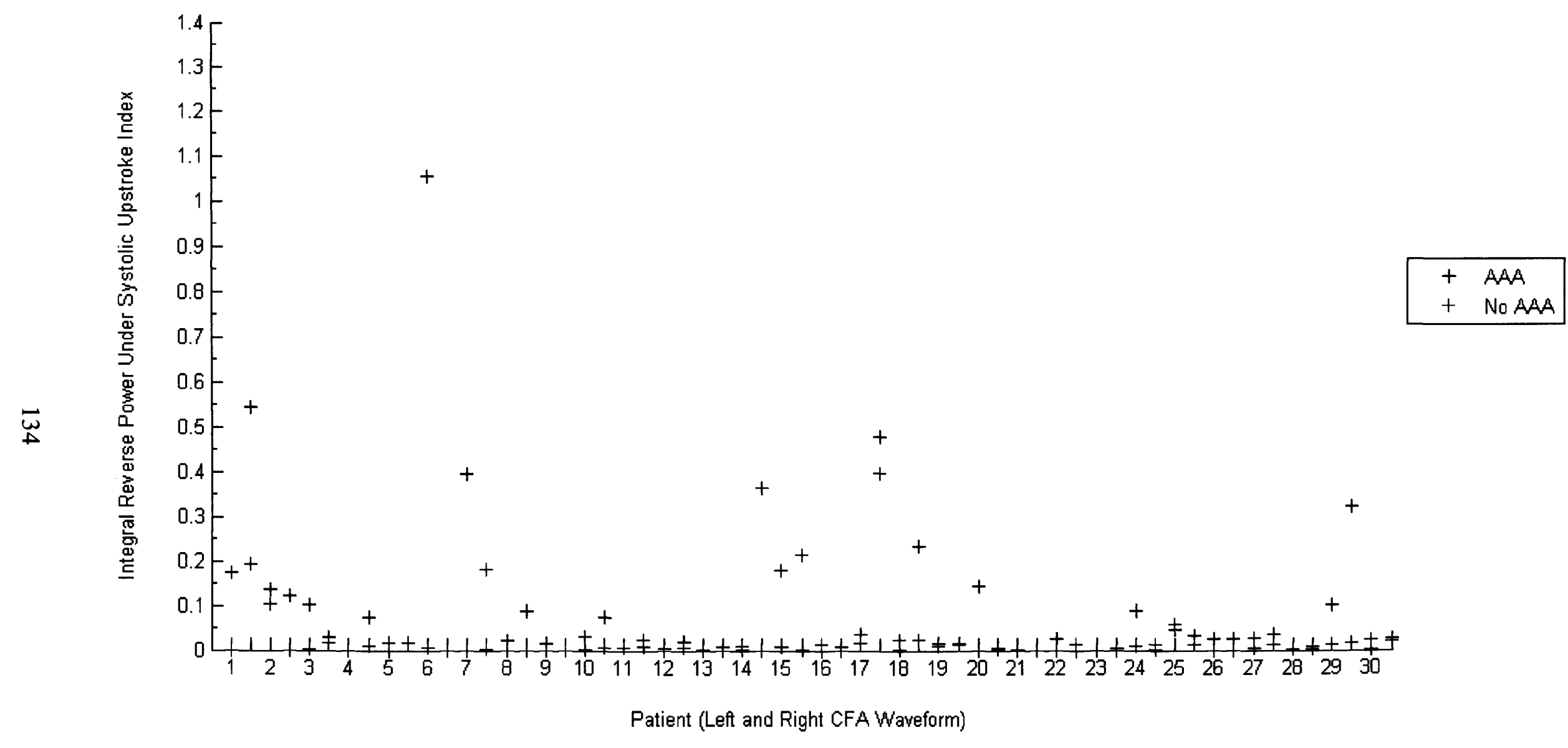

Figure 4.22: Integral of Reverse Power Under Systolic Upstroke Index plotted for the left and right CFA waveforms from the 30 AAA and 30 normal patients without significant atherosclerotic arterial disease 
The presence of reverse flow power under the systolic upstroke may possibly be explained by flow disturbances and vortices in the forward flow set up by an AAA. The overall direction of the bulk flow is forwards, but the vortices and flow disturbances have vectors in the reverse direction as the circulating vortices are carried forwards by the bulk flow. Therefore there will be a small amount of flow passing through the ultrasound beam with vectors in the reverse flow direction.

A range of threshold levels were tested on this index to determine the optimum level to discriminate between the AAA and normal waveforms. The results are given in Table 4.8. The threshold level of 0.025 provided a good level of separation with 25 out of the $60(41.7 \%)$ AAA waveforms and only 7 out of the $60(11.7 \%)$ normal waveforms above the threshold.

\begin{tabular}{|c|c|c|}
\hline Threshold Level & $\begin{array}{c}\text { Number of AAA } \\
\text { waveforms with index } \\
\text { above threshold }\end{array}$ & $\begin{array}{c}\text { Number of normal } \\
\text { waveforms with index } \\
\text { above threshold }\end{array}$ \\
\hline 0.025 & 25 & 7 \\
\hline 0.05 & 18 & 6 \\
\hline 0.10 & 14 & 5 \\
\hline 0.15 & 9 & 4 \\
\hline 0.20 & 7 & 2 \\
\hline
\end{tabular}

Table 4.8: Discrimination levels for the range of threshold levels tested for the Integral Reverse Power Under Systolic Upstroke index

The integral of the Reverse Power Under the Systolic Down-Stroke Index is plotted in Figure 4.23. 


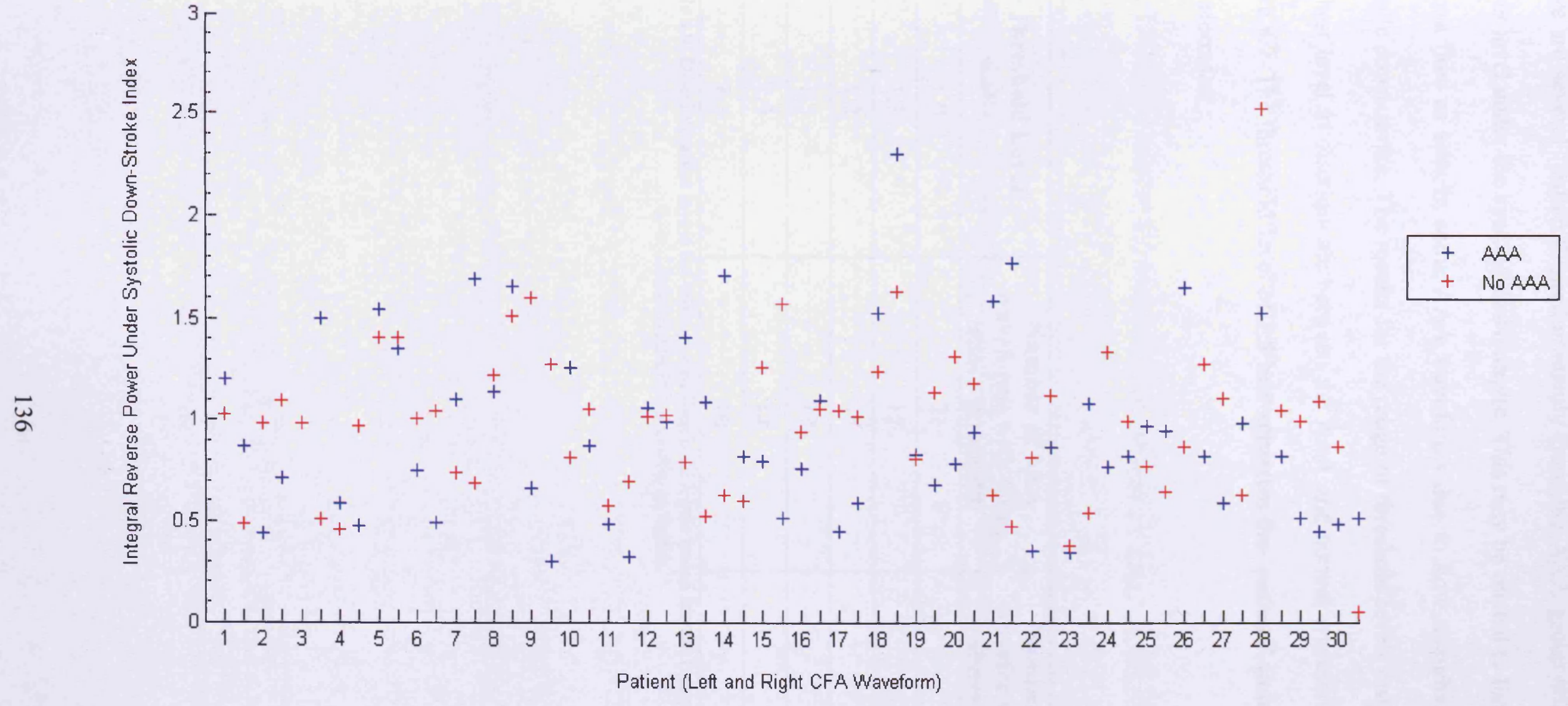

Figure 4.23: Integral of Reverse Power Under Systolic Down-Stroke Index plotted for the left and right CFA waveforms from the 30 AAA and 30 normal patients without significant atherosclerotic arterial disease 
There are several 'outliers', predominantly from the AAA group with an increased power level under the systolic down-stroke. This may be related to the early on-set of reverse flow as seen in some AAA waveforms due to flow disturbances during the systolic down-stroke. The results for the range of threshold levels tested to determine the best level to discriminate between the AAA and normal waveforms are given in Table 4.9. The threshold level of 1.5 best separates the 'outliers' from the rest of the waveforms.

\begin{tabular}{|c|c|c|}
\hline Threshold Level & $\begin{array}{c}\text { Number of AAA } \\
\text { waveforms with index } \\
\text { above threshold }\end{array}$ & $\begin{array}{c}\text { Number of normal } \\
\text { waveforms with index } \\
\text { above threshold }\end{array}$ \\
\hline 1.0 & 21 & 29 \\
\hline 1.1 & 17 & 18 \\
\hline 1.2 & 15 & 14 \\
\hline 1.3 & 13 & 9 \\
\hline 1.4 & 12 & 6 \\
\hline 1.5 & 10 & 5 \\
\hline
\end{tabular}

Table 4.9: Discrimination levels for the range of threshold levels tested for the Integral Reverse Power Under Systolic Down-Stroke index. 


\section{$\underline{4.5 .5}$ Elongation Indices}

The $5^{\text {th }}$ feature of waveform elongation is illustrated in Figure 4.24. To provide a measure for this feature the peak and pulse foot markers for the different phases of flow in each complete waveform cycle were used to calculate a number of different mean indices: Beat length, reverse flow length, waveform length, ratio of the waveform length to the beat length, ratio of the systolic peak length to the beat length, ratio of the reverse flow length to the beat length, ratio of the systolic peak length to the waveform length, ratio of the systolic peak length to the reverse flow length and the ratio of the reverse flow length to the waveform length.
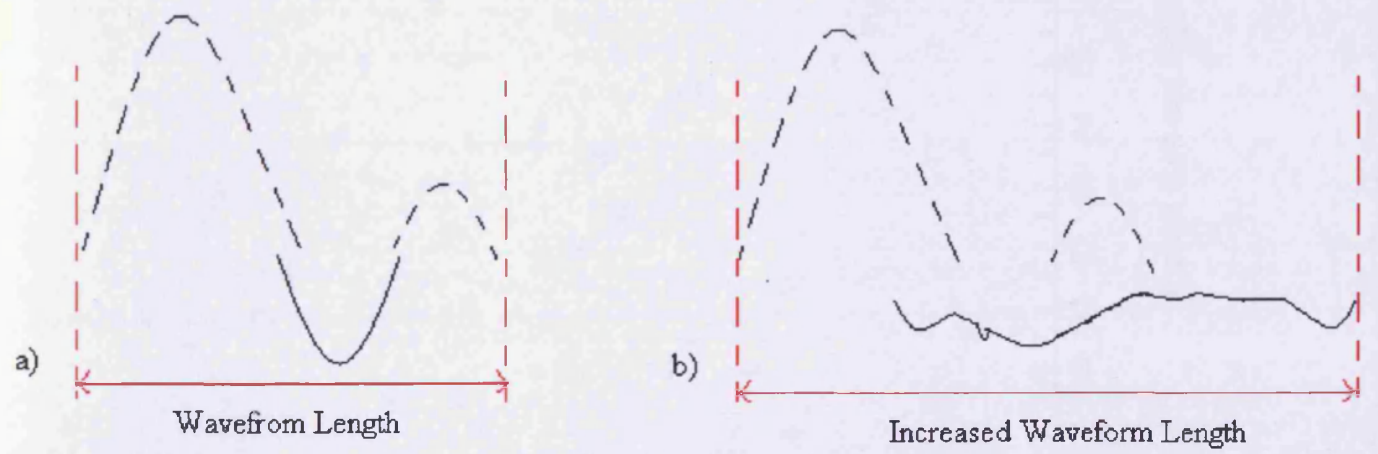

Figure 4.24: Illustration of a) normal wavelength and b) increased waveform length

The plots of all these indices for the left and right CFA waveforms from the 30 AAA and 30 normal patients were visually inspected. The index that provided the best separation between the AAA and normal groups was the Ratio of the Systolic Peak Length to the Reverse Flow Length Index which is plotted in Figure 4.25. 


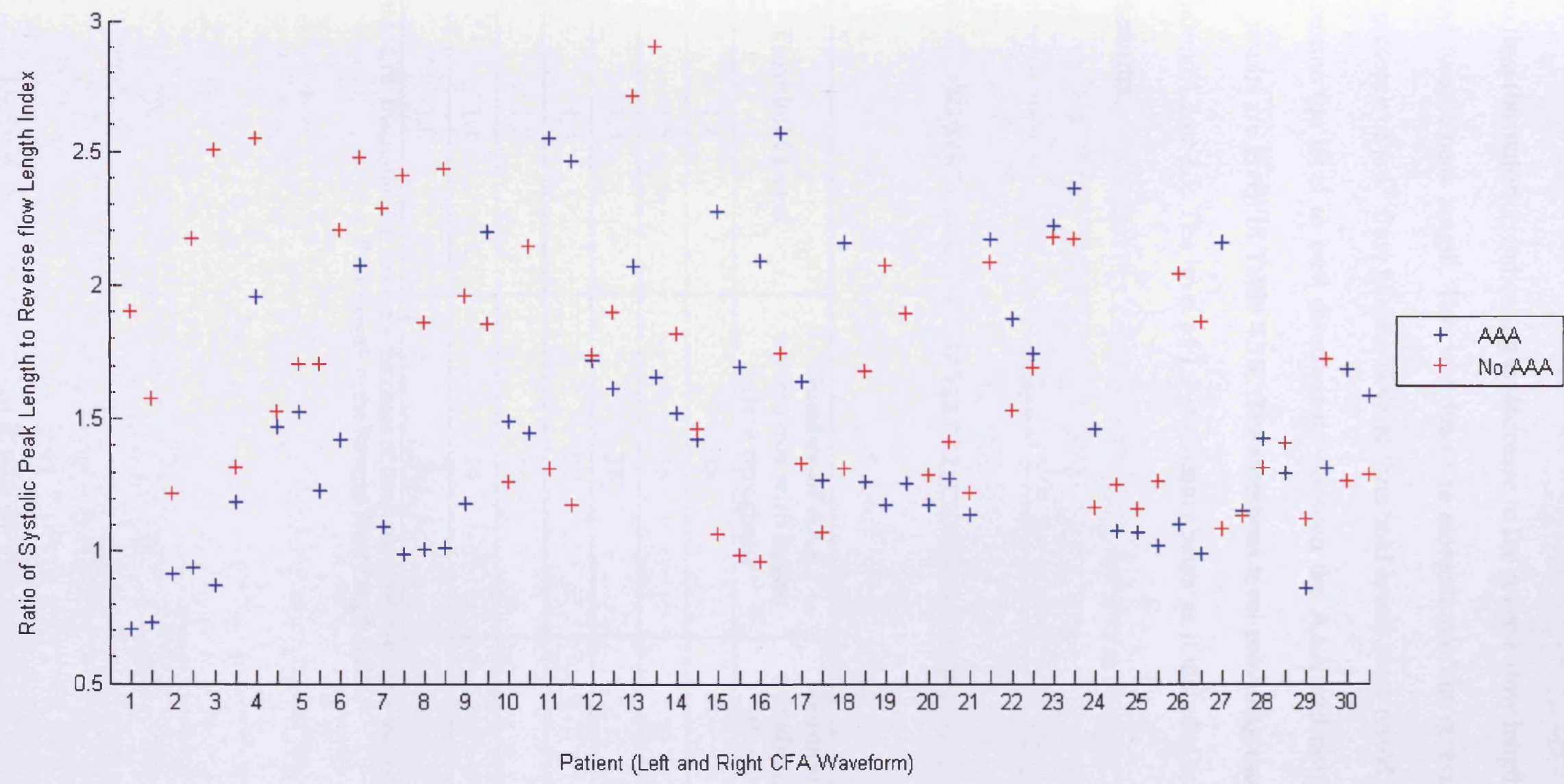

Figure 4.25: Mean Ratio of the Systolic Peak Length to the Reverse Flow Length Index plotted for the left and right CFA waveforms from the 30 AAA and 30 normal patients without significant atherosclerotic arterial disease 
There were several waveforms, predominantly in the AAA patient group with a lower ratio than the majority, indicating an increase in the reverse flow length in comparison to the systolic peak length. This was due to an elongation of the reverse flow phase or the presence of a $4^{\text {th }}$ flow phase. Several threshold levels were tested on this index to determine the level to best discriminate between the AAA and normal waveforms. The results are given in Table 4.10. The threshold level providing the best separation is around 1.2 or 1.3 . The level of 1.3 is possibly better as it included more of the AAA waveforms.

\begin{tabular}{|c|c|c|}
\hline Threshold Level & $\begin{array}{c}\text { Number of AAA } \\
\text { waveforms with index } \\
\text { below threshold }\end{array}$ & $\begin{array}{c}\text { Number of normal } \\
\text { waveforms with index } \\
\text { above threshold }\end{array}$ \\
\hline 1.6 & 38 & 29 \\
\hline 1.5 & 35 & 26 \\
\hline 1.4 & 28 & 24 \\
\hline 1.3 & 27 & 18 \\
\hline 1.2 & 21 & 10 \\
\hline 1.1 & 14 & 5 \\
\hline 1.0 & 8 & 2 \\
\hline
\end{tabular}

Table 4.10: Discrimination levels for the range of threshold levels tested for the Ratio of the Systolic Peak Length to the Reverse Flow Length Index 


\section{AAA and Normal Patients}

A summary of the 10 indices that were chosen to represent each of the 5 visual features is given in Table 4.11. The number of CFA waveforms included by the threshold levels that were set by visual inspection of the index plots are summarised in Figure 4.26.

\begin{tabular}{|c|c|}
\hline Visual Feature & MATLAB Index \\
\hline \multirow{3}{*}{$\begin{array}{c}\text { Increased Spectral Broadening } \\
\text { on Systolic Peak }\end{array}$} & $\begin{array}{c}\text { Maximum Systolic Down-Stroke } \\
\text { Bandwidth Index }\end{array}$ \\
\cline { 2 - 2 } $\begin{array}{c}\text { Spikes in systolic Down-Stroke } \\
\text { Profile }\end{array}$ & $\begin{array}{c}\text { Maximum Systolic Upstroke } \\
\text { Bandwidth Index }\end{array}$ \\
\hline \multirow{4}{*}{$\begin{array}{c}\text { Srregular Reverse Flow Pattern Spike Index } \\
\text { Simultaneous Forward and } \\
\text { Reverse Flow }\end{array}$} & Reverse Flow Fluctuation Index \\
\cline { 2 - 2 } & $\begin{array}{c}\text { Reverse to Systolic Height Ratio } \\
\text { Index }\end{array}$ \\
\cline { 2 - 2 } & $\begin{array}{c}\text { Systolic to Reverse Flow Time } \\
\text { Delay Index }\end{array}$ \\
\cline { 2 - 2 } & Systolic Upstroke Index \\
\cline { 2 - 2 } & $\begin{array}{c}\text { Integral Reverse Power Under } \\
\text { Systolic Down-Stroke Index }\end{array}$ \\
\hline \multirow{2}{*}{$\begin{array}{c}\text { Elongation of Waveform } \\
\text { Ratio of the Systolic Peak length } \\
\text { to the Reverse Flow Length Index }\end{array}$} \\
\hline
\end{tabular}

Table 4.11: Summary of the indices representing each of the 5 visual features. 


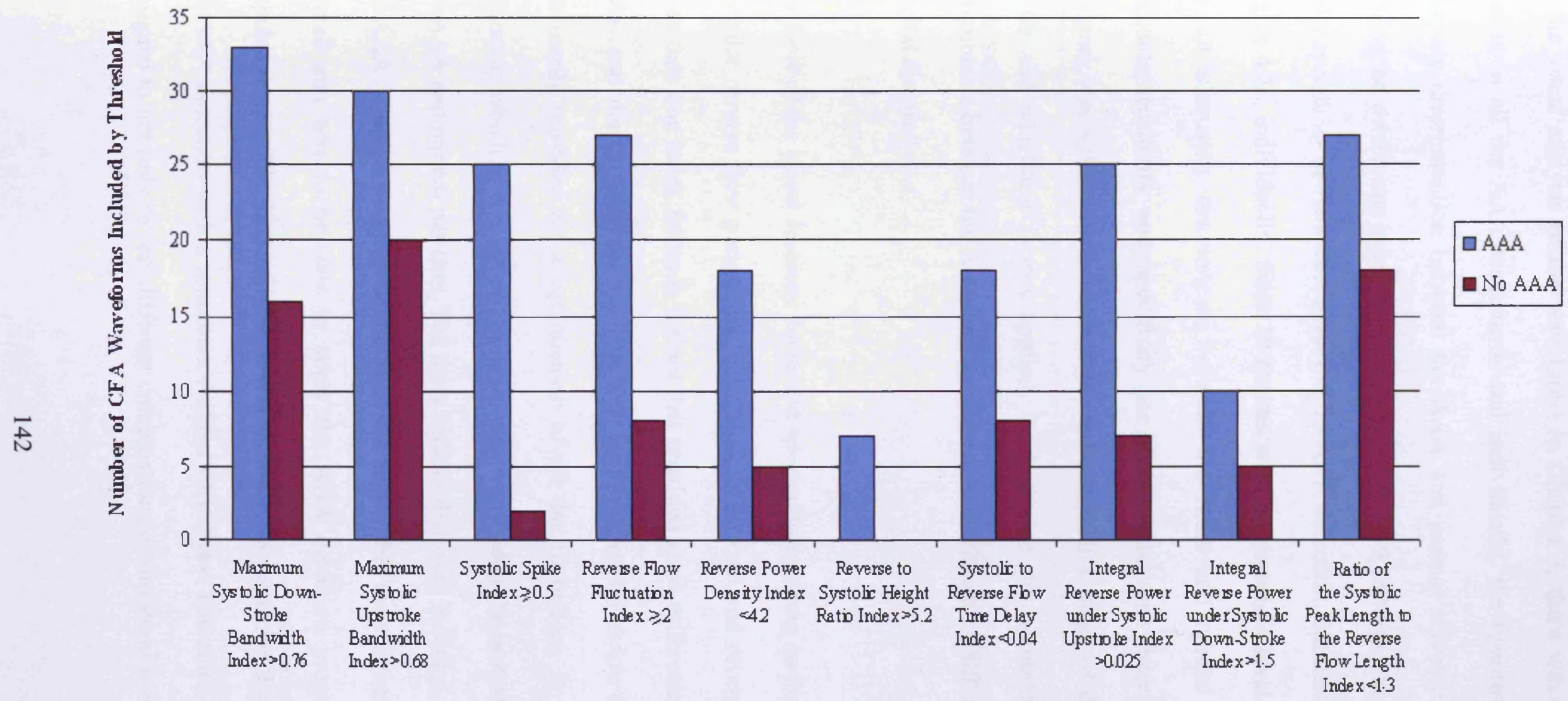

Index and Thues lold Level

Figure 4.26: Number of CFA waveforms from the $30 \mathrm{AAA}$ and 30 normal patients with no significant atherosclerotic arterial disease with an index value for each of the 10 Indices included by the corresponding threshold level 
In the visual analysis of the waveforms in Chapter 3, there was no single feature present in all the AAA waveforms and individually, the features did not provide adequate discrimination between the AAA and normal waveforms. However, by scoring the waveforms based on a combination of all 5 features a sensitivity of $93.3 \%$ and a specificity of $70 \%$ were achieved for AAA detection. Similarly, as illustrated in Figure 4.26, individually, these 10 indices with the threshold levels chosen visually, do not adequately discriminate between the AAA and normal waveforms with adequate sensitivity and specificity for AAA detection. Therefore a MATLAB program was written to combine these indices, with a range of different threshold levels and weighting factors applied, to determine the optimum combination to discriminate between the AAA and normal patients without significant atherosclerotic arterial disease.

For three of the visual features: Increased spectral broadening on the systolic peak, an irregular reverse flow pattern and simultaneous forward and reverse flow there was more than one index for each feature that provided good differentiation between the AAA and normal waveforms. The best performance for AAA detection was not necessarily provided by a combination of all the 10 indices. It was necessary to determine which of the indices in combination provided the best separation between the AAA and normal patients. The more indices that were included, the more specific the AAA detection program would be to the data within the study group. Because the overall aim was to be able to apply the AAA detection program to a general population, possibly for screening, it was necessary to determine the best combination for AAA detection with a minimum number of indices. Therefore, the program was designed to not only to test different combinations of threshold levels and weighting 
factors, but also different combinations with different numbers of the indices included.

The method by which the MATLAB based program operated was similar to the process used to detect AAA by visual analysis of the CFA waveforms in Chapter 3 . For each patient in the AAA and normal study groups, the indices representing each of the visual features were calculated individually for the right and left CFA waveforms. Each waveform was then allocated a score for each of the indices depending upon where the calculated index value lay in relation to the threshold level set for the index. The total scores for the ten indices for each waveform were summed. For each patient the total waveform scores for the left and right leg were then summed to give a total patient score. Once the total patient scores had been calculated for all the $30 \mathrm{AAA}$ and normal patients, the total scores were subjected to threshold levels to determine the threshold level to best distinguish between the AAA and normal patients.

A summary of how the MATLAB program combined the different indices, threshold levels and weighting factors is given in Figure 4.27. The threshold levels (T1-T10) and weighting factors (W1-W10) for each of the 10 individual indices were entered manually at the start of the program. The ranges of the threshold levels that were tested were taken from the values given in Tables 4.1-4.10. The weighting factors were set to 1,2 , or 0 . A weight of 2 increased the importance of the index in the combination, and a weight of 0 in effect, removed the index from the combination. The left and right CFA waveform data files for each of the 30 AAA and 30 normal patients were loaded automatically and in sequence into the program. The initial data 
processing was performed on each waveform file to reduce noise, extract the maximum frequency envelopes for the forward and reverse flow components, calculate the positions of the forward and reverse flow phase markers, and calculate the minimum frequency envelopes, bandwidth envelopes and power envelopes. The 10 indices were then calculated individually for each waveform and compared to the set threshold levels. For the Maximum Systolic Down-Stroke Bandwidth Index, Maximum Systolic upstroke Bandwidth Index, Systolic Spike Index, Reverse Flow Fluctuation Index, Reverse to Systolic Height Ratio Index, Integral Reverse Power Under Systolic Upstroke index, Integral Reverse Power Under Systolic Down-Stroke Index and Ratio of the Systolic Peak Length to the Reverse flow Length Index a score of 1 was awarded if the waveform index level lay above the threshold or 0 if it lay below. For the Reverse Power Density Index and Systolic to Reverse Flow TimeDelay Index, a score of 1 was awarded if the waveform index lay below the threshold or 0 if it lay above. In effect, the threshold levels were used to determine whether the visual feature represented by the index was present in the waveform or not. For each individual waveform, the score for each index was then multiplied by the corresponding weighting factor that was entered at the start of the program. The weighted scores for the 10 indices were summed to give a total score for each individual waveform. The waveform scores for the left and right leg of each patient were then summed to give a total patient score. To determine the best threshold level to place on the patient score to provide the best sensitivity and specificity to AAA detection, the total score for each patient and the number of patients in each group with total scores above threshold levels of $\geq 1, \geq 2$ and $\geq 3$ were output at the end of the program. 


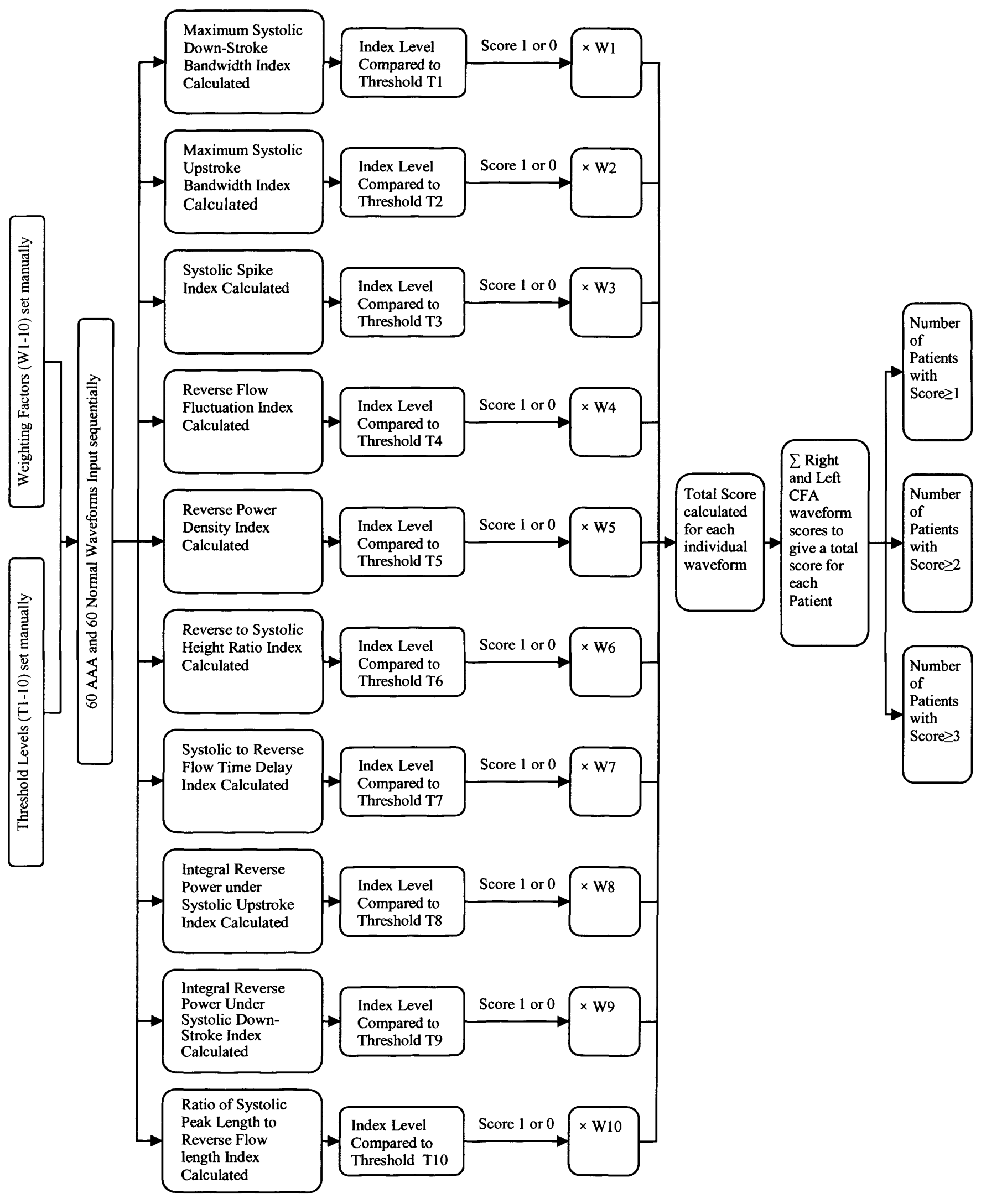

Figure 4.27: Summary of how the MATLAB program combines the indices, threshold levels and weighting factors 
A large number of different combinations and permutations of the 10 indices, threshold levels and weighting factors were systematically tested. The best level of separation between the patients in the AAA and normal study groups without significant atherosclerotic disease was achieved by the combination of 6 indices to represent the 5 visual features with the corresponding threshold levels and weighting factors given in Table 4.12. The remaining 4 indices: Maximum Systolic Upstroke Bandwidth Index, Reverse Flow Fluctuation Index, Ratio of the Systolic Peak Length to the Reverse Flow Length Index and Systolic to Reverse Flow Time-Delay Index did not improve on the sensitivity and specificity for AAA detection when included in the combination and were therefore disregarded. All indices apart from the systolic spikes index were not weighted. The Systolic Spike Index was weighted by a factor of 2 , because visually (and objectively), the feature of spikes on the systolic down-stroke was a very dominant feature in the AAA waveforms. 


\begin{tabular}{|c|c|c|c|}
\hline Visual Feature & Index & Threshold Level & Weighting Factor \\
\hline $\begin{array}{c}\text { Increased } \\
\text { Spectral } \\
\text { Broadening } \\
\end{array}$ & $\begin{array}{l}\text { Maximum Systolic } \\
\text { Down-Stroke } \\
\text { Bandwidth Index } \\
\end{array}$ & $>0.76$ & 1 \\
\hline $\begin{array}{c}\text { Spikes on Systolic } \\
\text { Down-stroke }\end{array}$ & Systolic Spike Index & $\geq 0.5$ & 2 \\
\hline $\begin{array}{c}\text { Irregular Reverse } \\
\text { Flow Pattern / } \\
\text { Waveform } \\
\text { Elongation } \\
\end{array}$ & $\begin{array}{l}\text { Reverse Power } \\
\text { Density Index }\end{array}$ & $<4.2$ & 1 \\
\hline $\begin{array}{l}\text { Irregular Reverse } \\
\text { Flow Pattern }\end{array}$ & $\begin{array}{l}\text { Reverse to Systolic } \\
\text { Height Ratio Index }\end{array}$ & $>0.52$ & 1 \\
\hline \multirow{2}{*}{$\begin{array}{l}\text { Simultaneous } \\
\text { Forward and } \\
\text { Reverse Flow }\end{array}$} & $\begin{array}{c}\text { Integral Reverse } \\
\text { Power under } \\
\text { Systolic Upstroke } \\
\text { Index } \\
\end{array}$ & $>0.025$ & 1 \\
\hline & $\begin{array}{c}\text { Integral Reverse } \\
\text { Power under } \\
\text { Systolic Down- } \\
\text { Stroke Index } \\
\end{array}$ & $>1.5$ & 1 \\
\hline
\end{tabular}

Table 4.12: Indices with corresponding threshold levels and weighting factors providing the best discrimination between the AAA and normal study group patients without significant atherosclerotic arterial disease.

\subsection{AAA Detection Program Results}

\subsubsection{AAA Detection Program Study Groups' Results}

The total scores that were output by the program for each individual patient in the AAA and normal patient study groups for this combination are plotted in Figures $4.28 \mathrm{a}$ and $4.28 \mathrm{~b}$ respectively.

The number of patients in each group with a total score of $\geq 1, \geq 2$ and $\geq 3$ are plotted in Figure 4.29. 


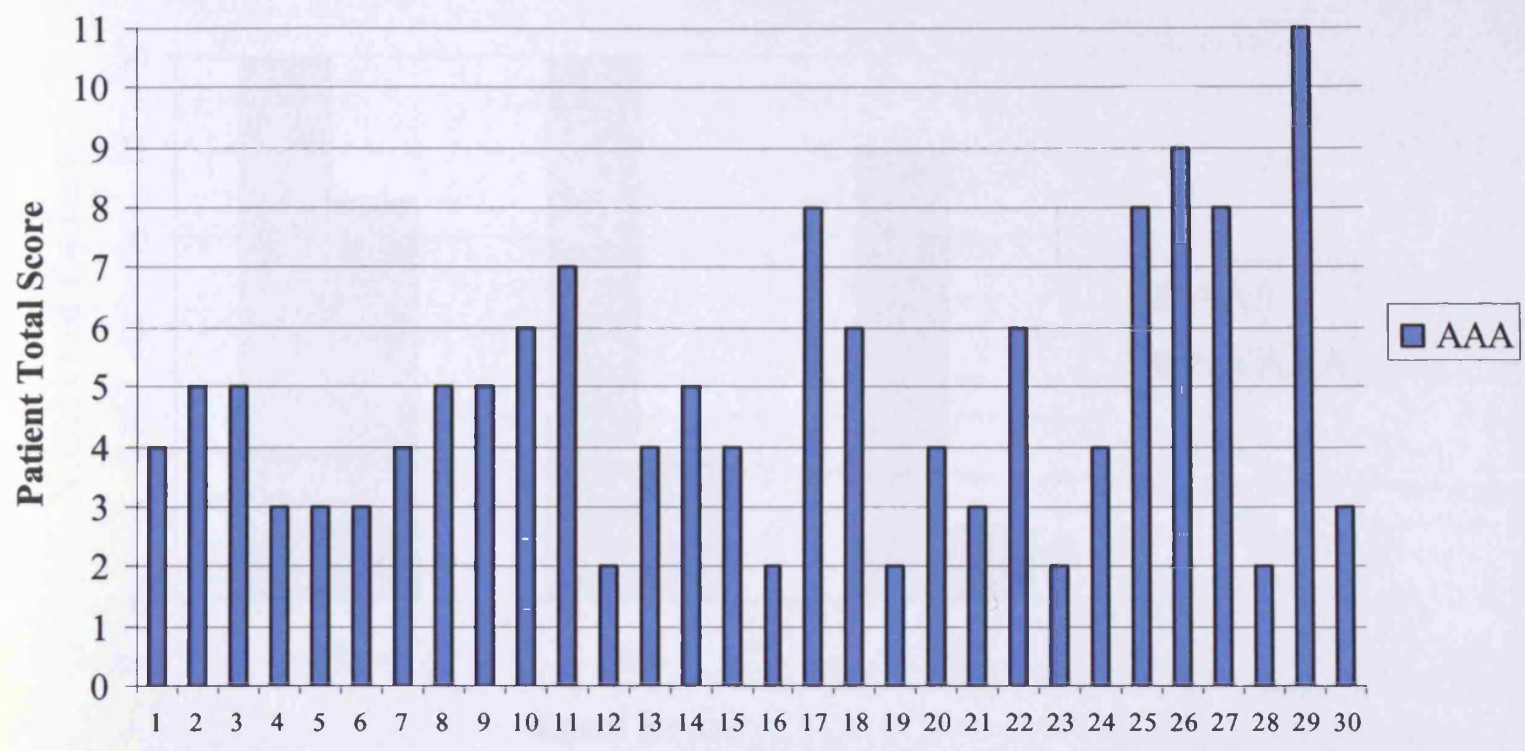

Patient

Figure 4.28a: Total Patient Scores for the 30 AAA patients in the no significant atherosclerotic disease study group

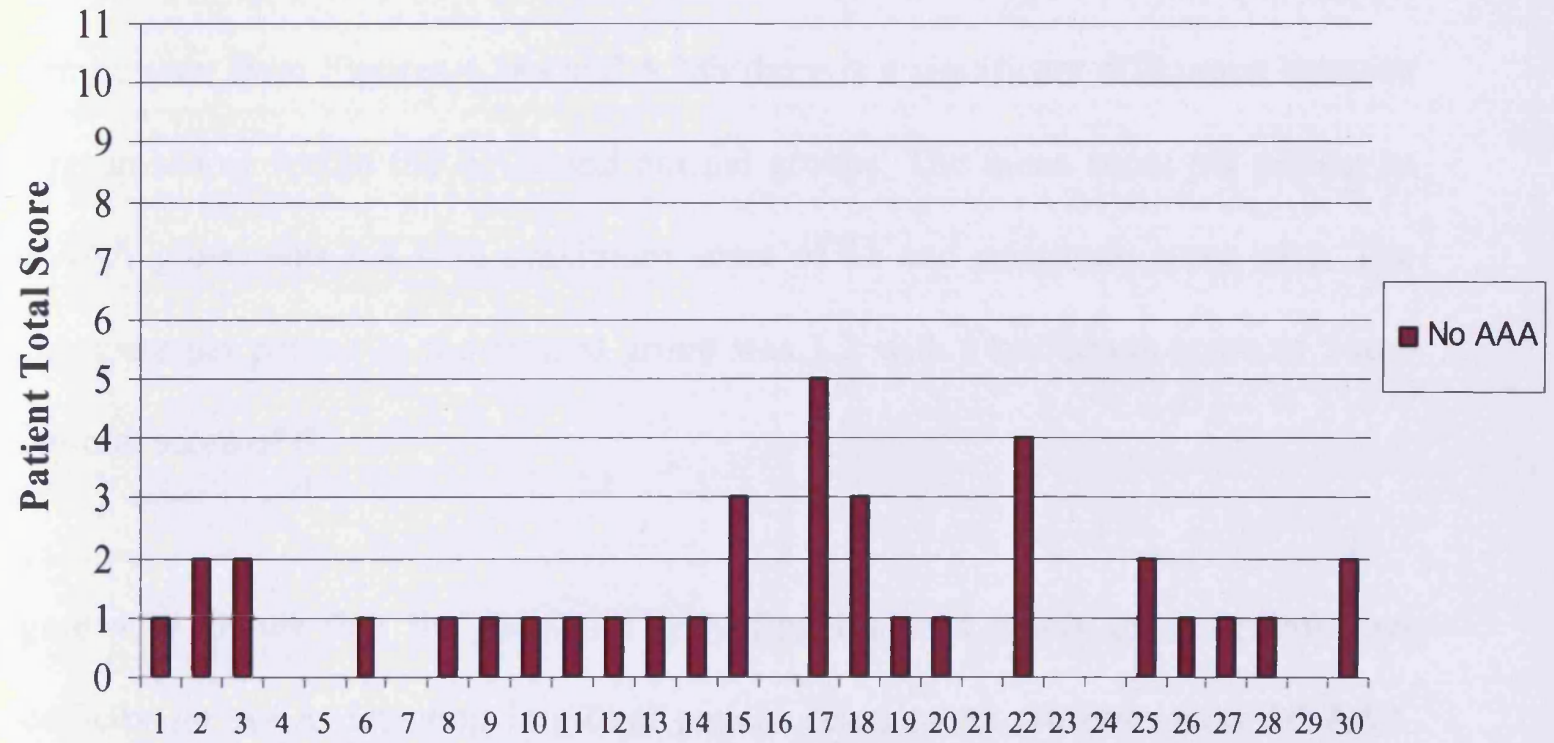

Patient

Figure 4.28b: Total Patient Scores for the 30 normal patients in the no significant atherosclerotic disease study group 


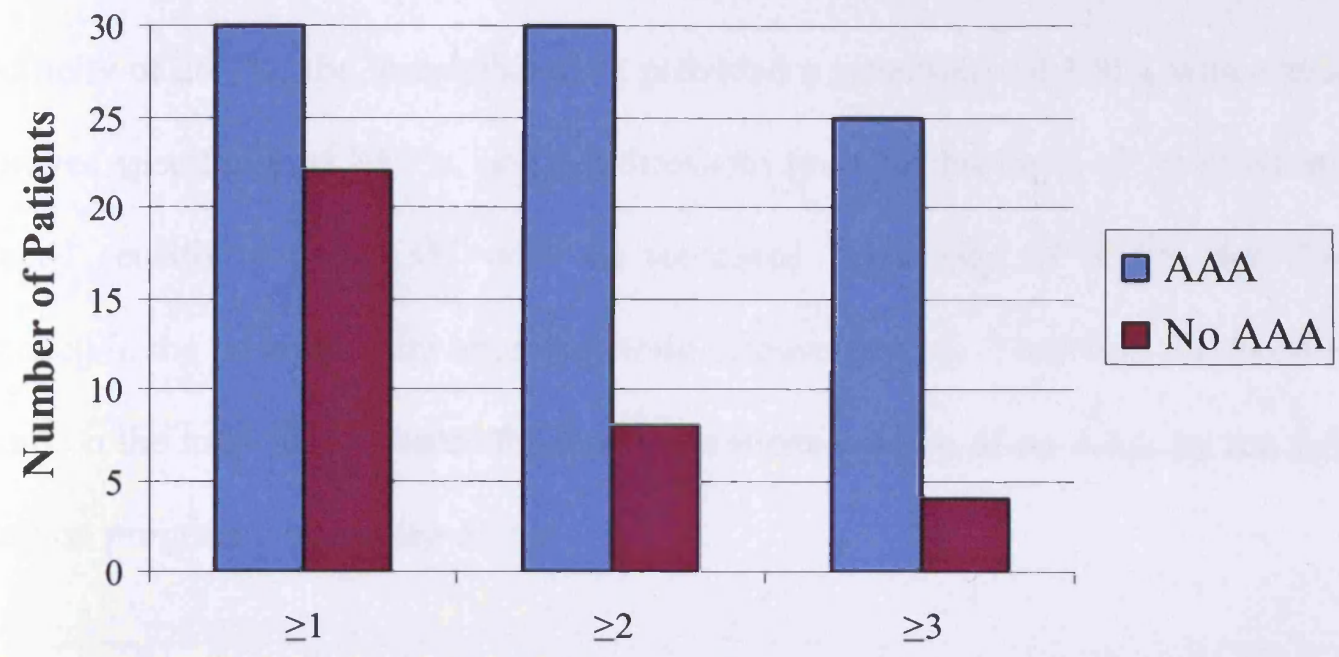

Total Score

Figure 4.29: Number of AAA and normal patients in the no significant atherosclerotic disease study groups with a Total Patient Score of $\geq 1, \geq 2$ and $\geq 3$.

\subsubsection{AAA Detection Program Study Groups' Results: Discussion}

It can be seen from Figures $4.28 \mathrm{a}$ and $4.28 \mathrm{~b}$ there is a significant difference between the mean scores within the AAA and normal groups. The mean score per patient in the AAA group was 4.8 with maximum score of 11 and minimum score of 2 . The mean score per patient in the normal group was 1.2 with a maximum score of 5 and minimum score of 0 .

Figure 4.29 shows that the threshold providing the best levels of sensitivity and specificity for AAA detection is a total patient score of $\geq 2.30$ out of the 30 AAA patients had a total patient score $\geq 2$ and 25 out of the 30 AAA patients had a total patient score $\geq 3$. For the normal patient group, 22 patients out of 30 had a total patient score $\geq 1,8$ out of 30 had a total patient score $\geq 2$ and 4 out of 30 had a total patient 
score $\geq 3$. Therefore, the threshold of $\geq 1$ provided a sensitivity of $100 \%$ with a specificity of $26.7 \%$, the threshold of $\geq 2$ provided a sensitivity of $100 \%$ with a much improved specificity of $73.3 \%$, and the threshold level of threshold of $\geq 3$ provided a reduced sensitivity of $83.3 \%$ with an increased specificity of $86.6 \%$ for AAA detection in the no significant atherosclerotic disease groups. Therefore the threshold placed on the total patient score for a positive identification of an AAA by the AAA detection program was a score of $\geq 2$.

\subsubsection{Response of AAA Detection Program to Blind Test Groups}

The AAA detection program provided very good differentiation between the 30 AAA and 30 normal patients in the study groups used to the select the combination of the indices, threshold levels and weighting factors used in the program. In order to test the ability of the program to diagnose AAA from a blind data set, the previously unseen waveforms from the test groups containing $7 \mathrm{AAA}$ and 11 normal patients without significant atherosclerotic disease (separated from the cohort at the start of the study, summarised in Table A1.2, Appendix 1 and Table A2.2, Appendix 2) were subjected to the AAA detection program.

The 6 indices: 1) Maximum Systolic Down-Stroke Bandwidth Index, 2) Systolic Spike Index, 3) Reverse Power Density Index, 4) Reverse to Systolic Height Ratio Index, 5) Integral Reverse Power under Systolic Upstroke index, and 6) Integral Reverse Power Under Systolic Down-Stroke Index are plotted for the left and right CFA waveforms for the $7 \mathrm{AAA}$ and 11 normal test group patients in Figures $4.30 \mathrm{a}$ to 4.30g. The dotted lines indicate the threshold levels set by the program. 


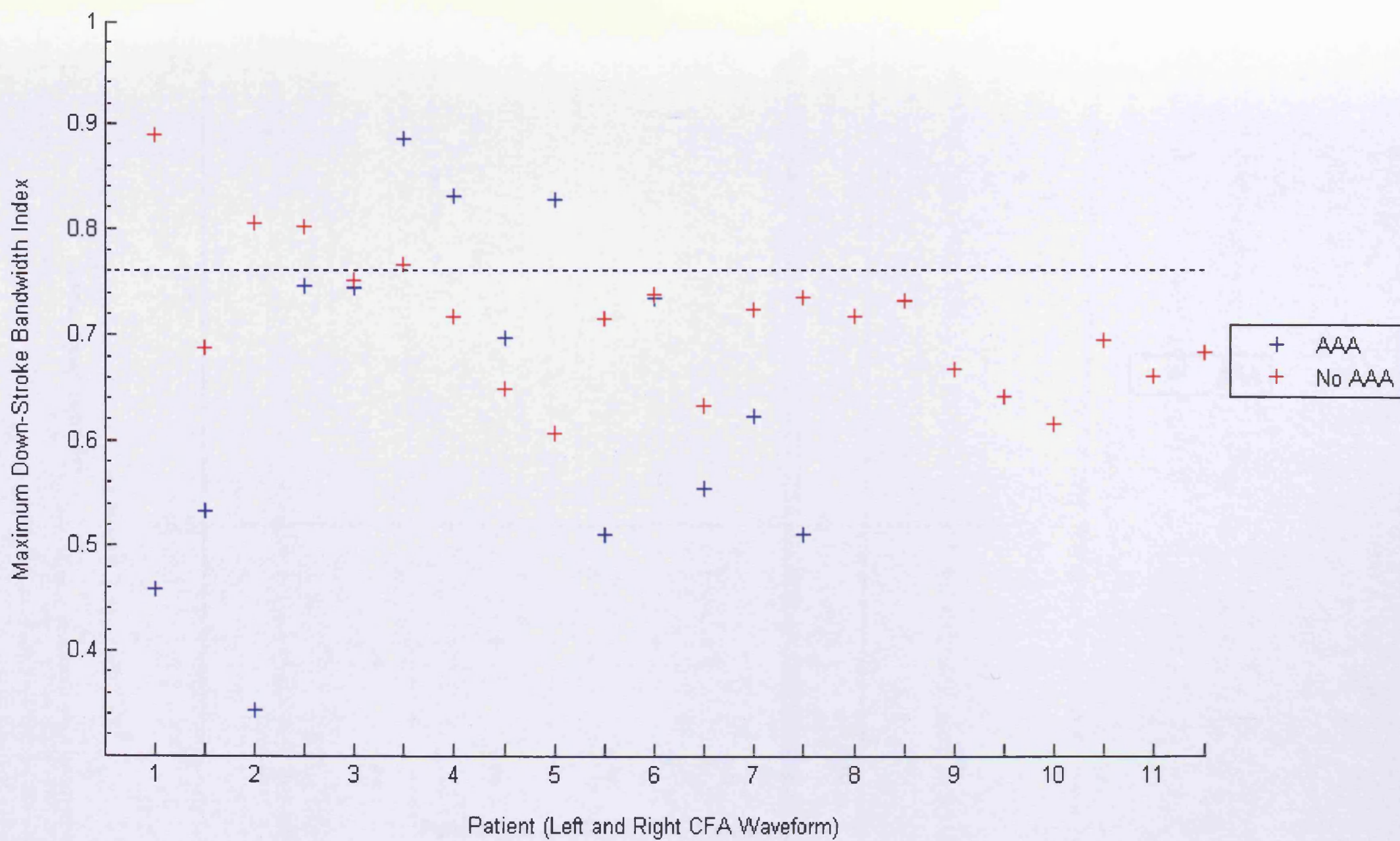

Figure 4.30a: Maximum Systolic Down-Stroke Bandwidth Index plotted for the left and right CFA waveforms for the 7 AAA and 11 normal patients in the no significant atherosclerotic disease test groups. 


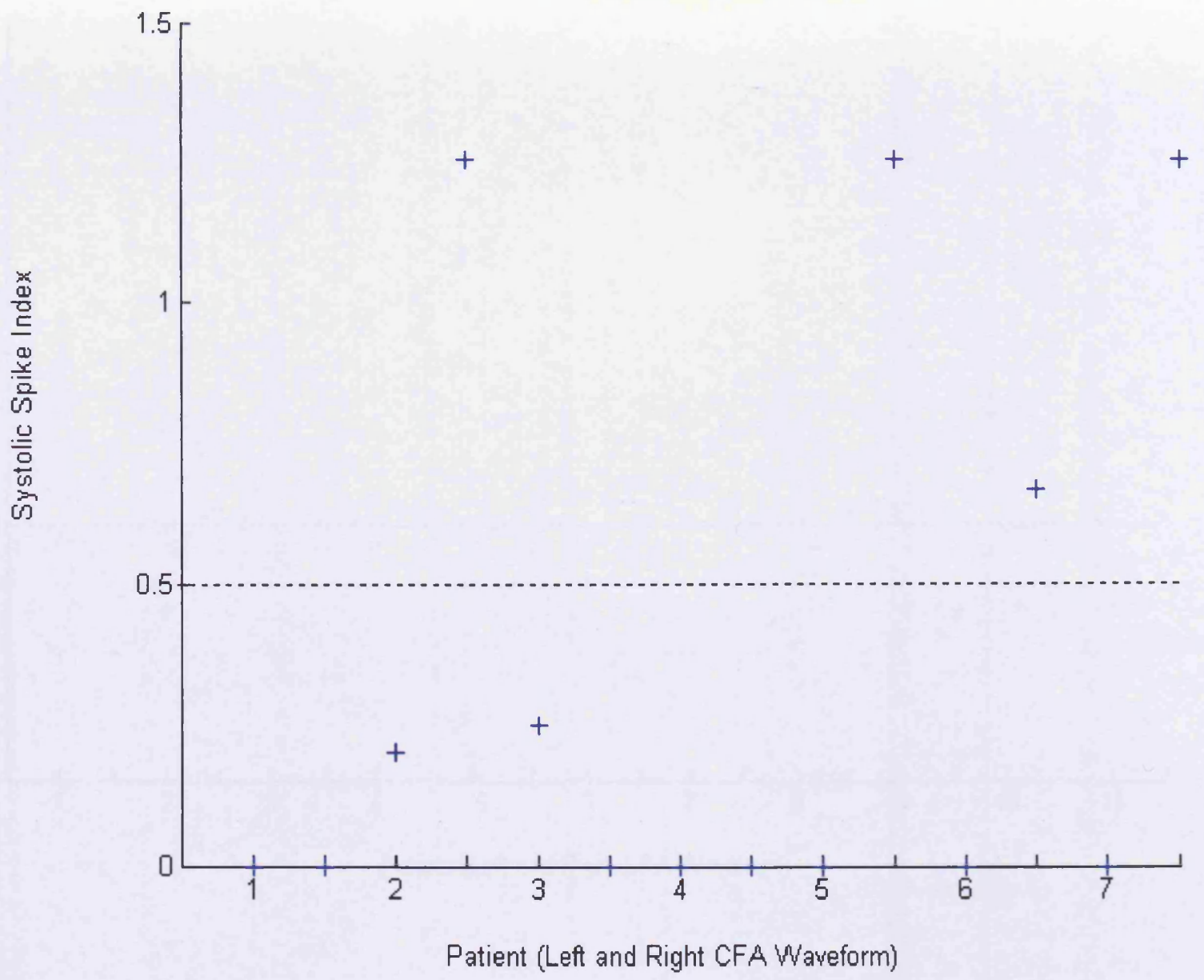

Figure 4.30b: Systolic Spike Index plotted for the left and right CFA waveforms for the 7 AAA patients in the no significant atherosclerotic disease test group. 


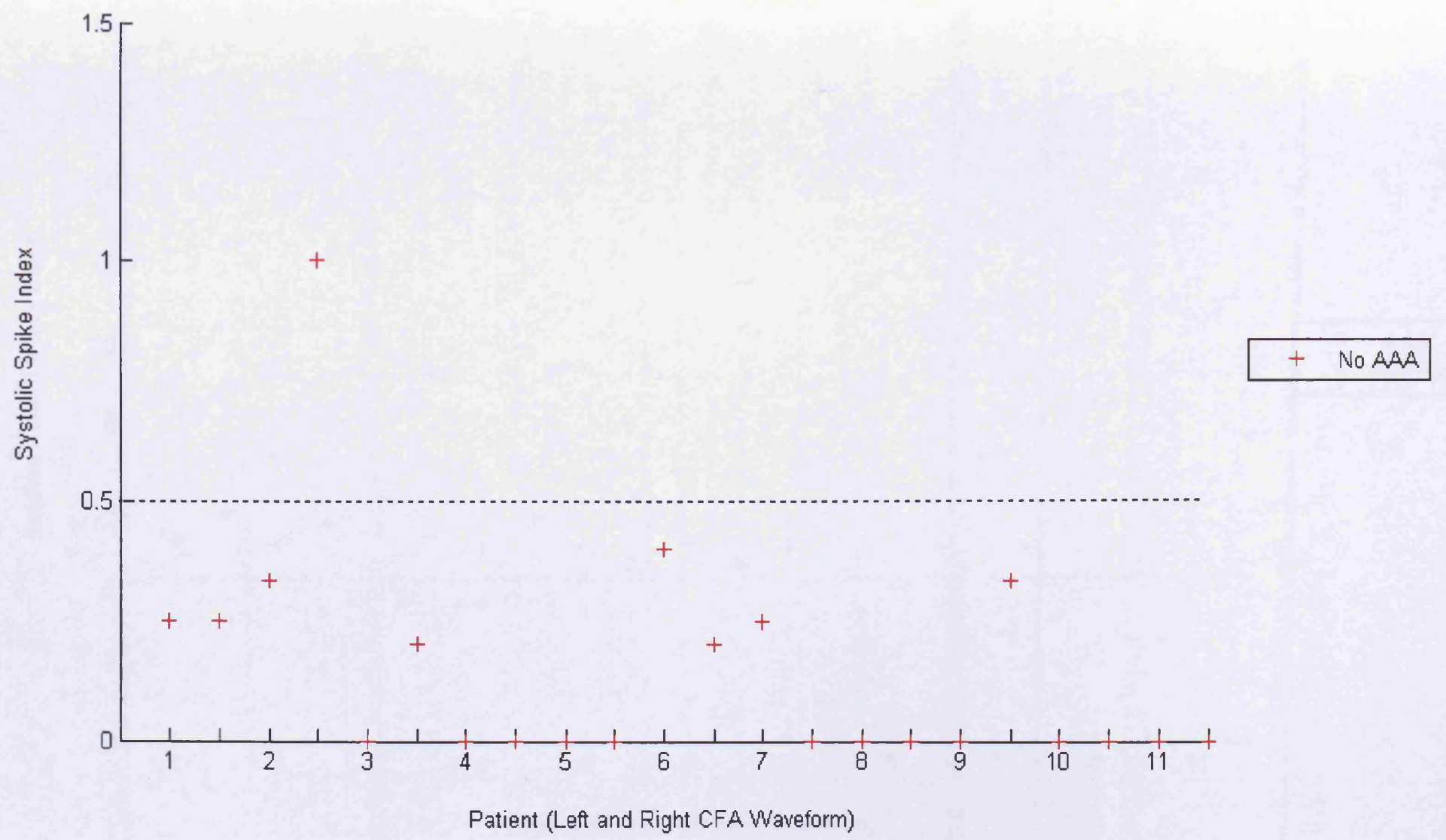

Figure 4.30c: Systolic Spike Index plotted for the left and right CFA waveforms for the 11 normal patients in the no significant atherosclerotic disease test group. 


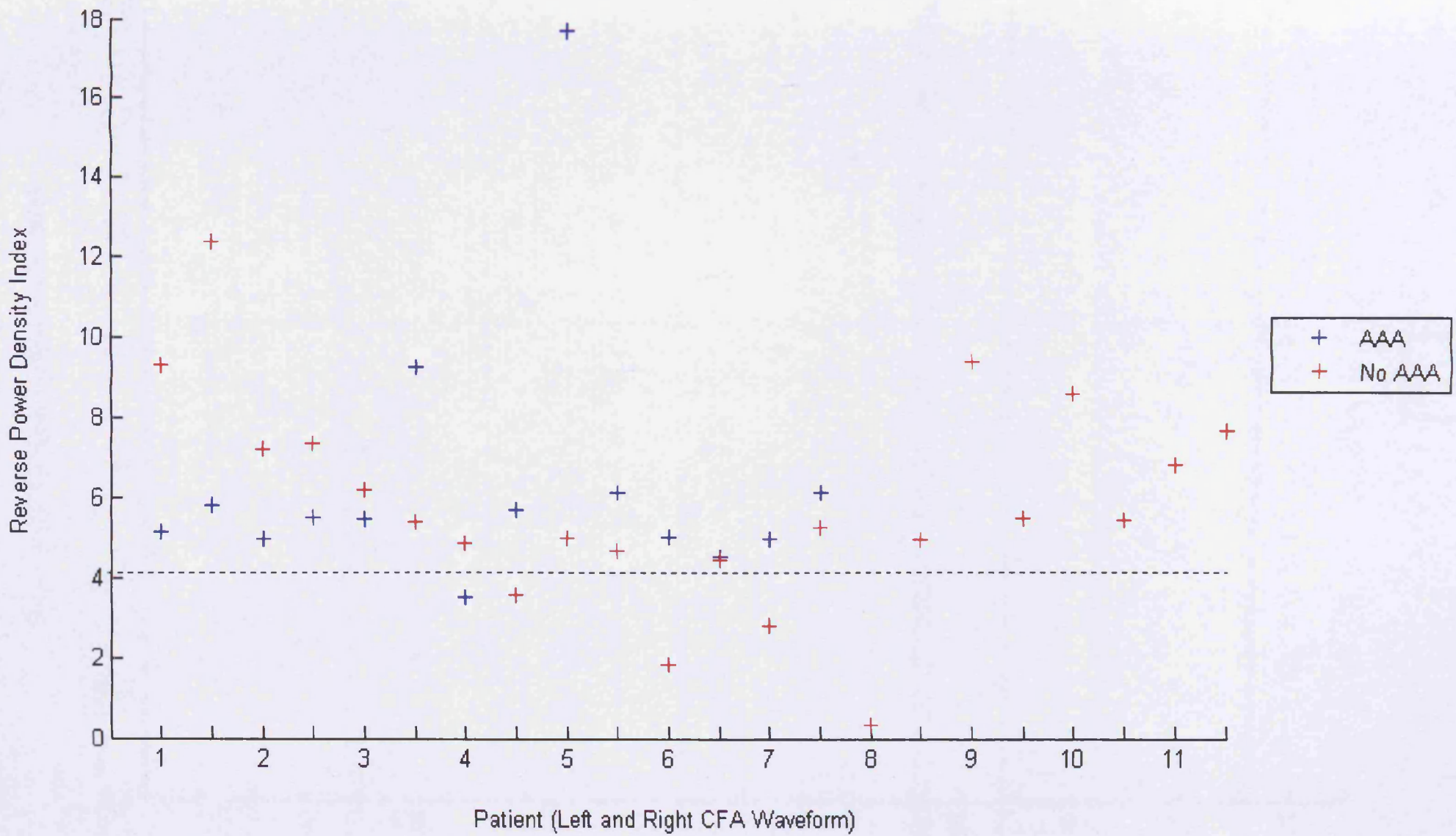

Figure 4.30d: Reverse Power Density Index plotted for the left and right CFA waveforms for the 7 AAA and 11 normal patients in the no significant atherosclerotic disease test groups. 


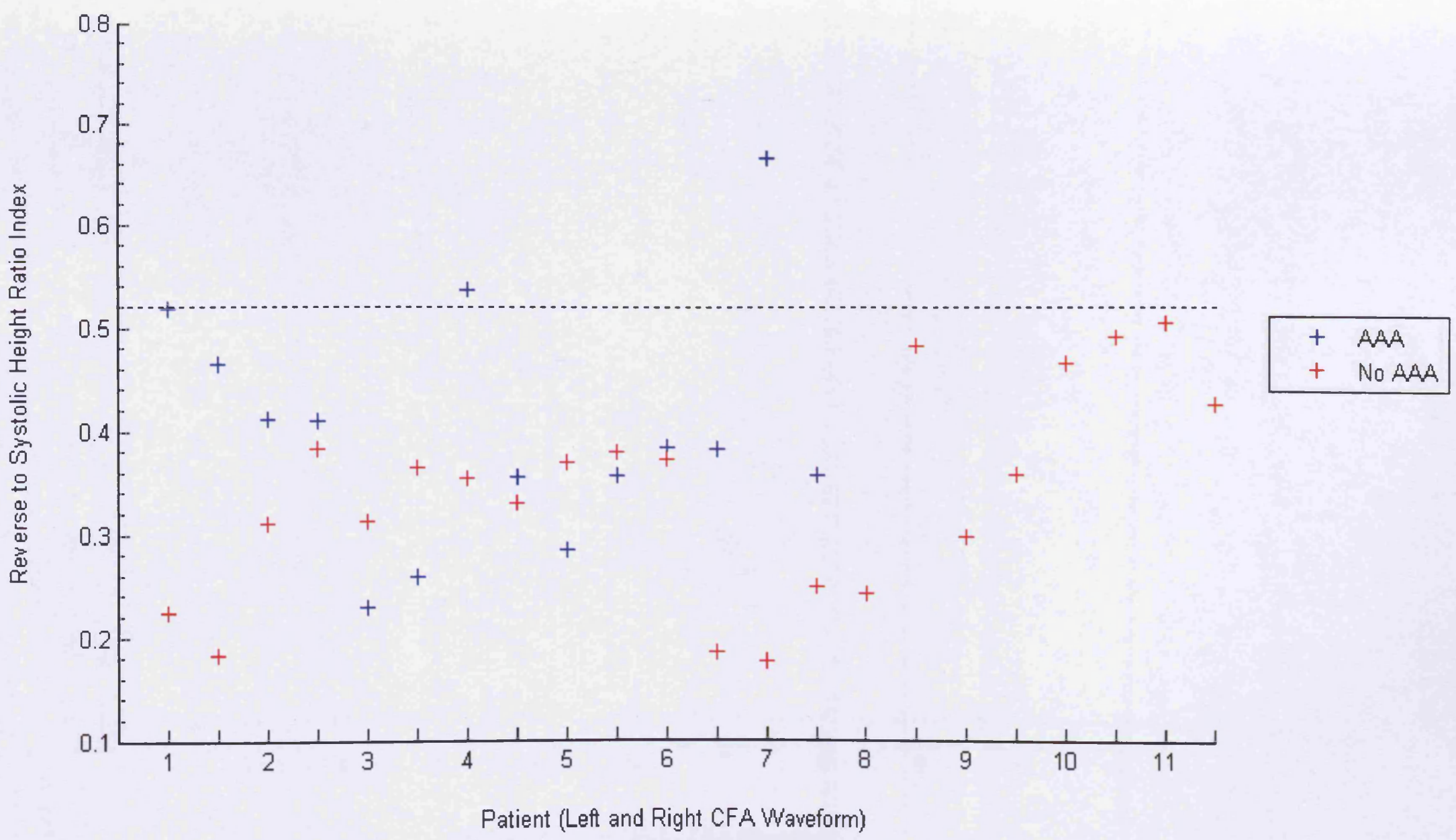

Figure 4.30e: Reverse to Systolic Height Ratio Index plotted for the left and right CFA waveforms for the 7 AAA and 11 normal patients in the no significant atherosclerotic disease test groups. 


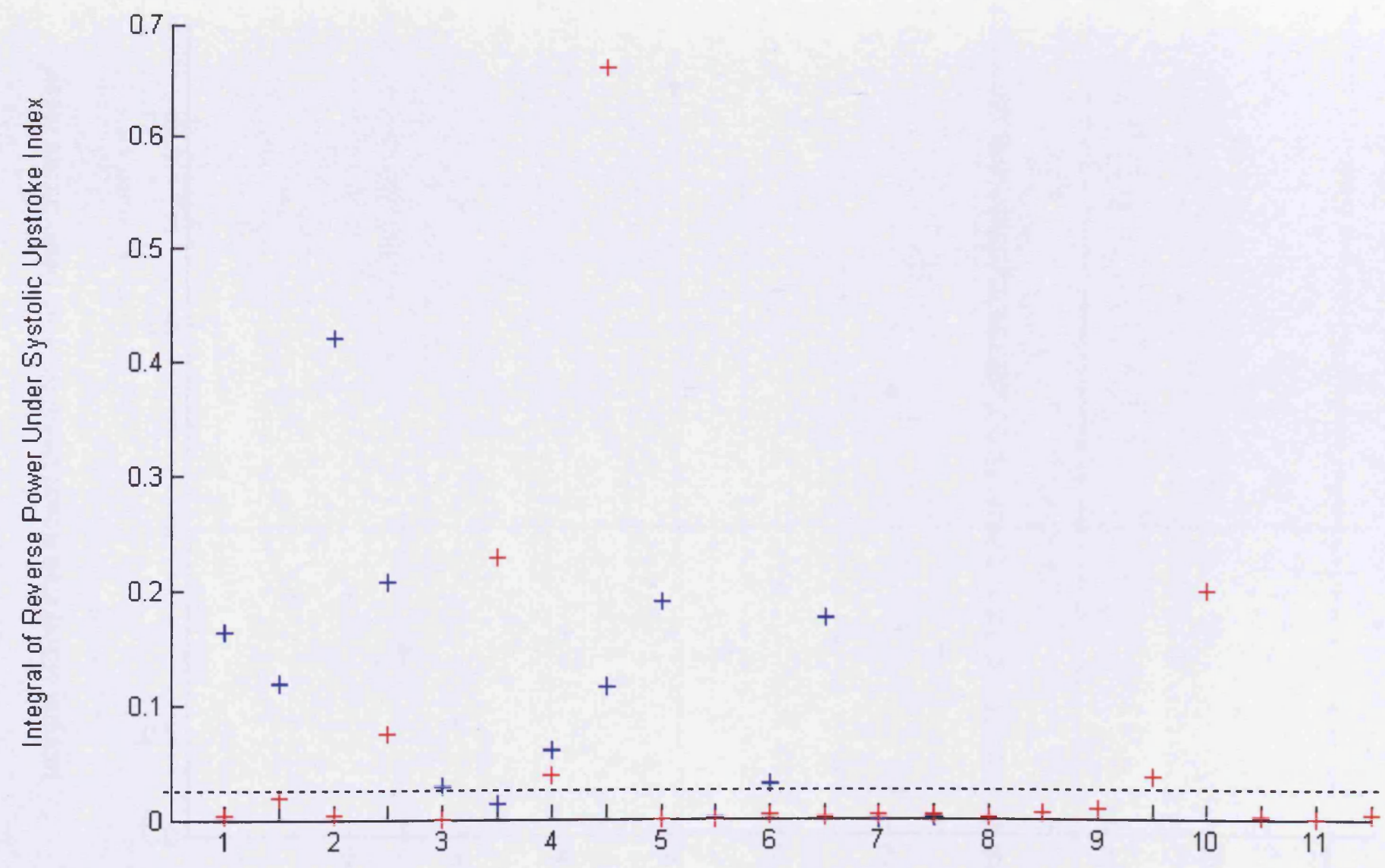

\section{Patient (Left and Right CFA Waveform)}

Figure 4.30f: Integral of Reverse Power Under the Systolic Upstroke Index plotted for the left and right CFA waveforms for the 7 AAA and 11 normal patients in the no significant atherosclerotic disease test groups. 


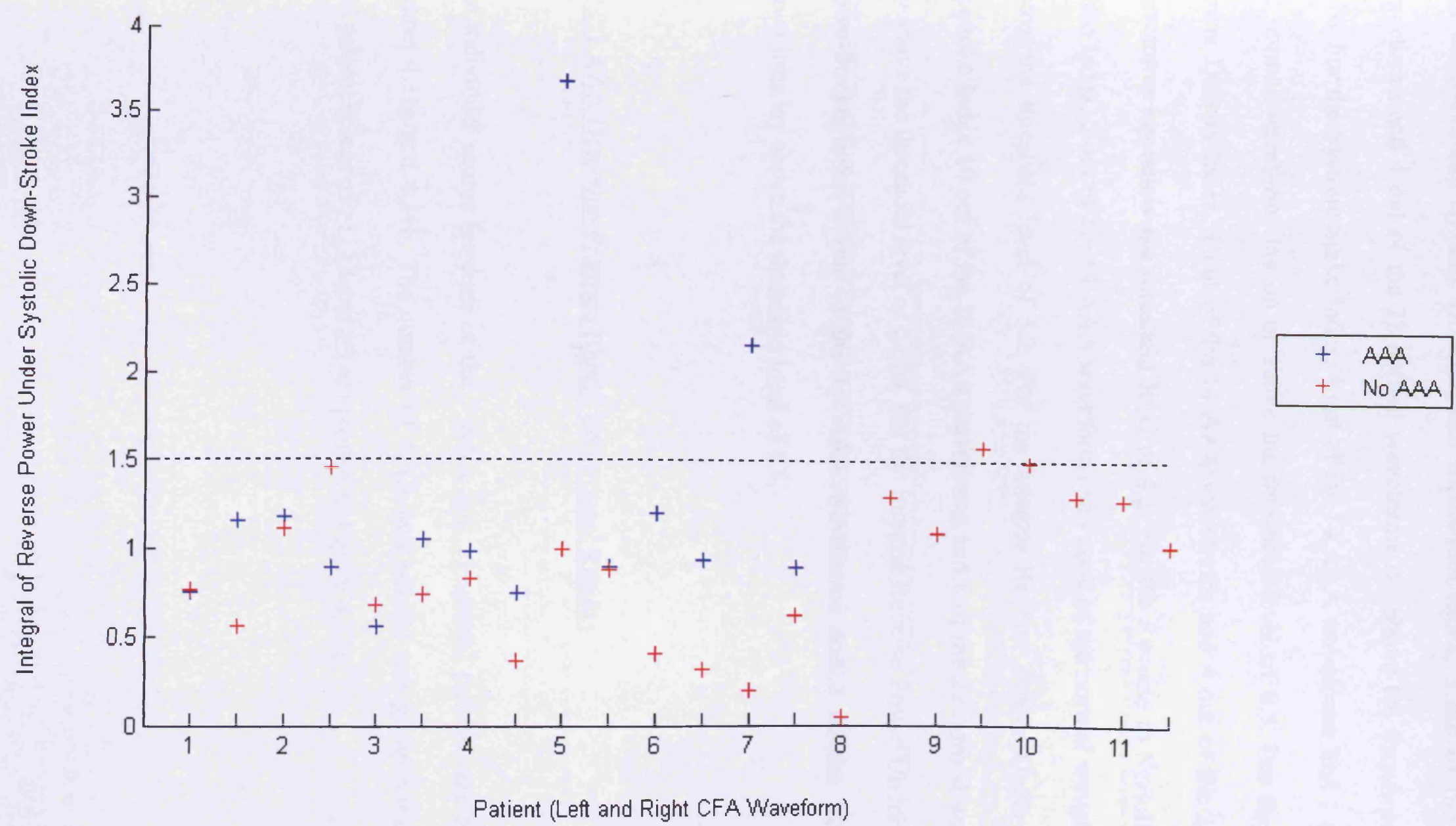

Figure 4.30g: Integral of Reverse Power Under the Systolic Down-Stroke Index plotted for the left and right CFA waveforms for the 7 AAA and 11 normal patients in the no significant atherosclerotic disease test groups. 
For the Maximum Systolic Down-Stroke Bandwidth Index, 3 out of the 14 AAA waveforms and 4 out of the 22 normal waveforms lay above the threshold level of 0.76. For the Systolic Spike Index, 4 out of the 14 AAA waveforms and 1 out of the 22 normal waveforms lay on or above the threshold level of 0.5 . For the Reverse Power Density Index, 1 out of the 14 AAA waveforms and 4 out of the 22 normal waveforms lay below the threshold level of 4.2. For the Reverse to Systolic Height Ratio Index, 2 out of the 14 AAA waveforms and none of the normal waveforms lay above the threshold level of 5.2. For the Integral Reverse Power Under Systolic Upstroke Index 10 out of the 14 AAA waveforms and 6 of the 22 normal waveforms lay above the threshold level of 0.025 . For the Integral Reverse Power Under Systolic Down-Stroke Index 2 out of the 14 AAA waveforms and 1 of the 22 normal waveforms lay above the threshold level of 1.5.

\subsubsection{AAA Detection Program Blind Test Groups' Results}

The individual scores for each of the $7 \mathrm{AAA}$ and 11 normal patients are plotted in Figures 4.31a and 4.31b. The number of AAA and normal test group patients with total patient scores of $\geq 1, \geq 2$ and $\geq 3$ are plotted in Figure $4.31 \mathrm{c}$. 


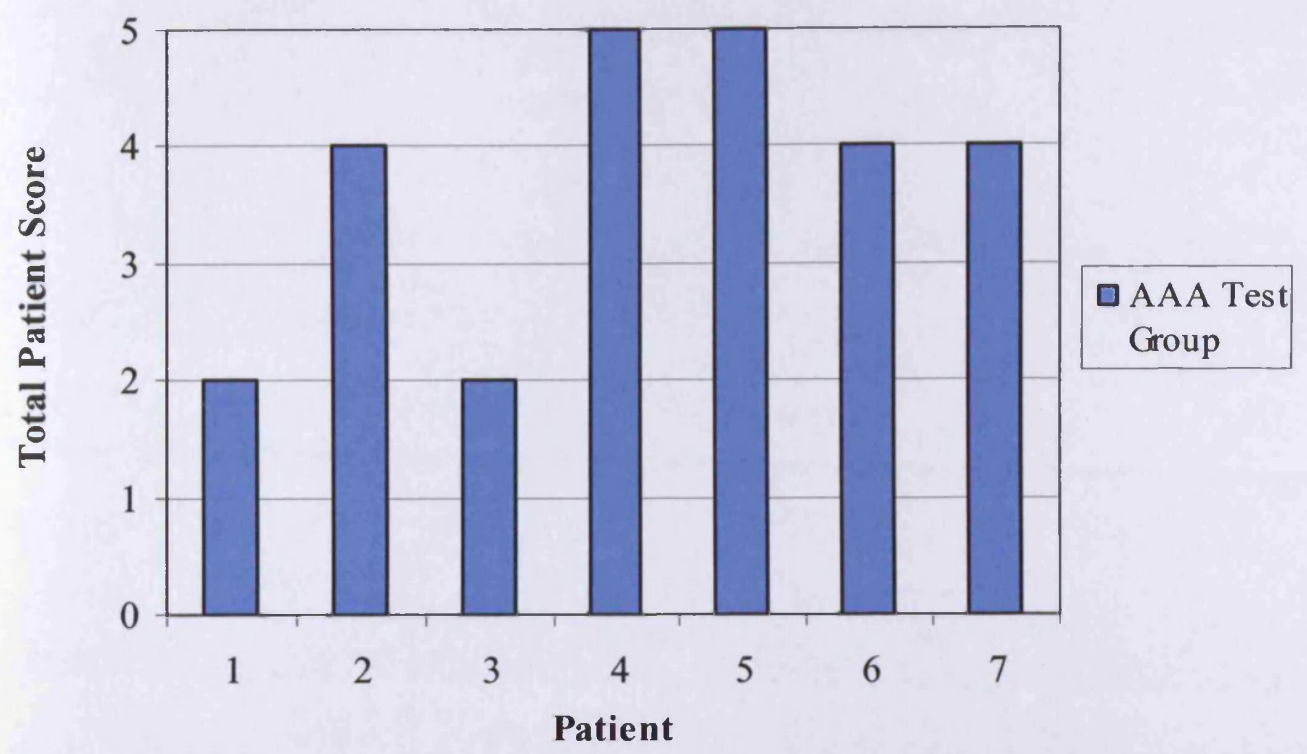

Figure 4.31a: Total Patient Scores for the 7 AAA test group patients with no significant atherosclerotic disease

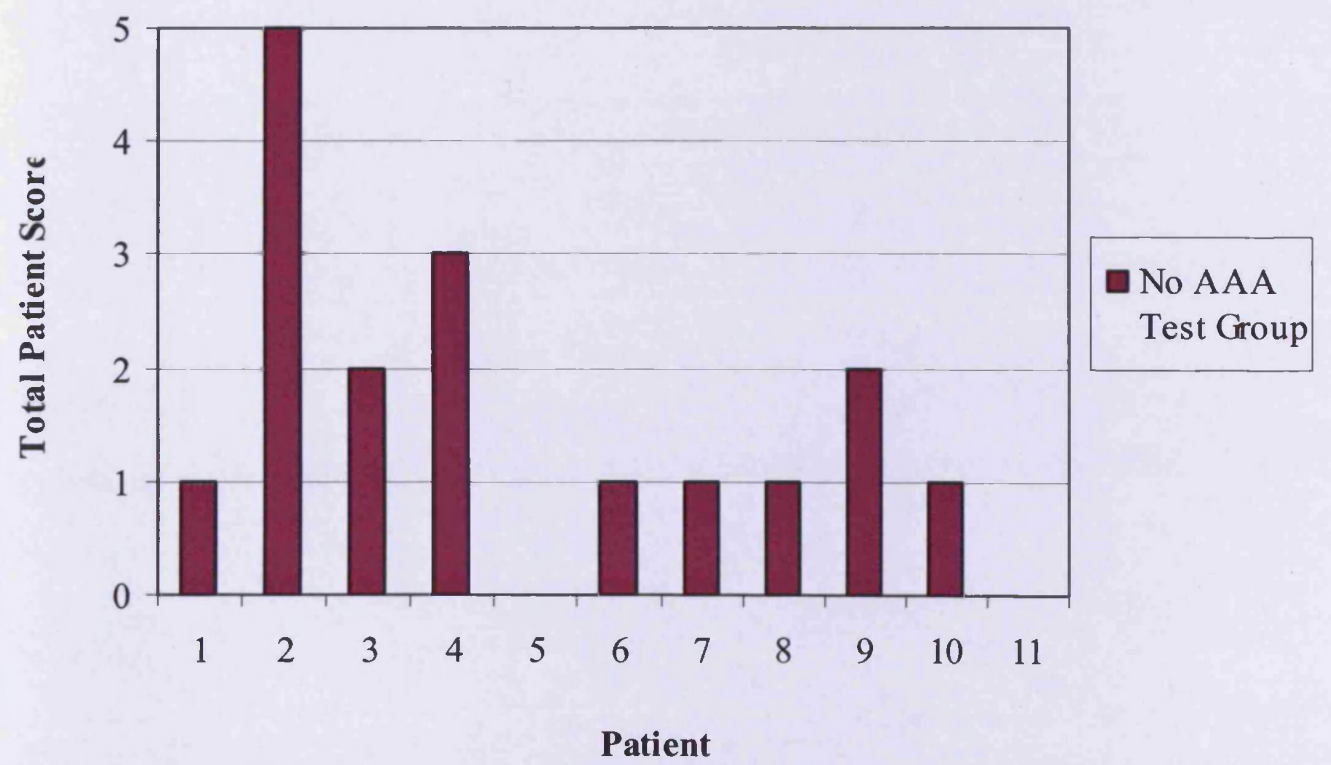

Figure 4.31b: Total Patient Scores for the 11 normal test group patients with no significant atherosclerotic disease 


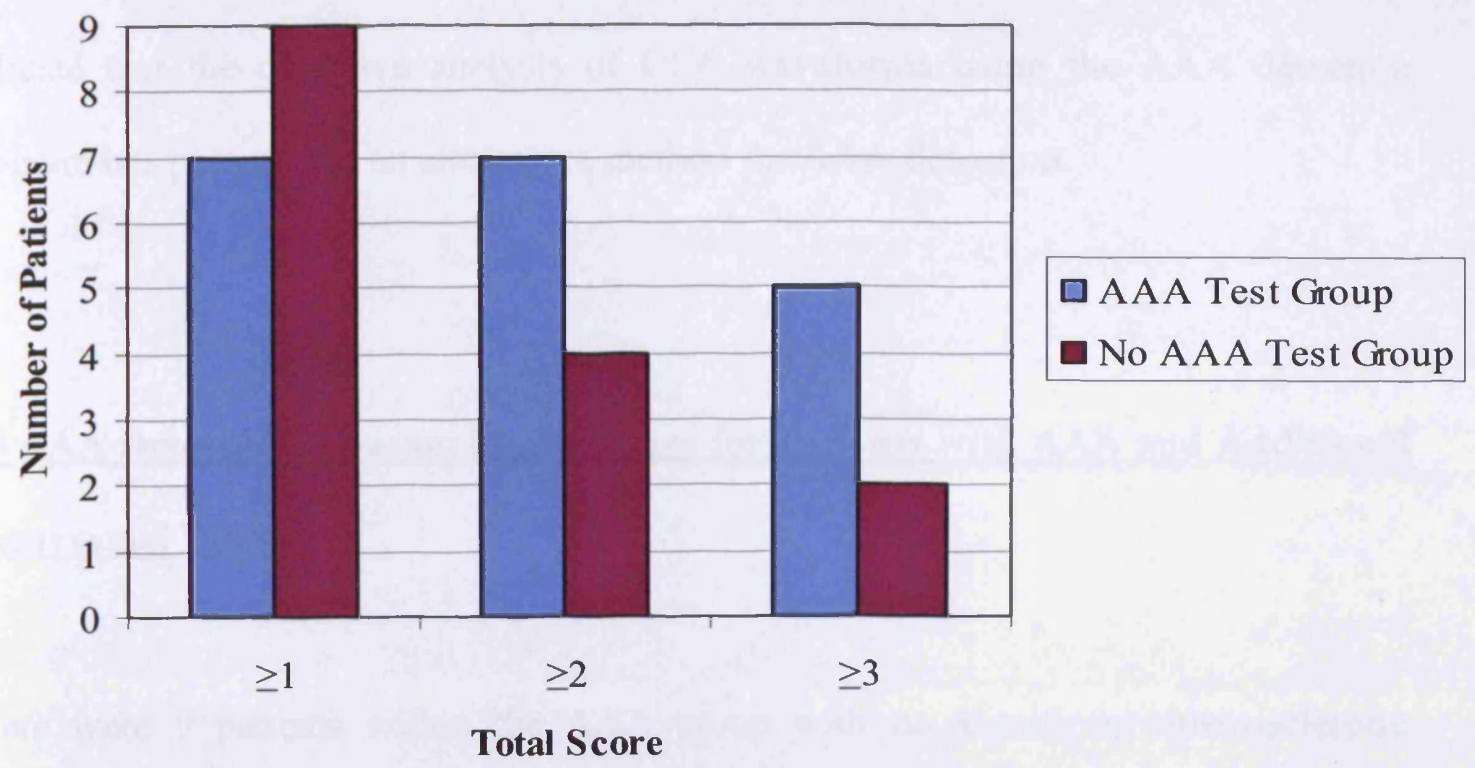

Figure 4.31c: Number of AAA $(n=7)$ and normal $(n=11)$ patients in the no significant atherosclerotic disease test groups with total patient scores of $\geq 1, \geq 2$ and $\geq 3$

\subsubsection{AAA Detection Program Blind Test Groups' Results: Discussion}

It can be seen from Figures $4.31 \mathrm{a}$ and $4.31 \mathrm{~b}$ that there is a significant difference between the mean patient scores in the AAA and normal test group. The mean score per patient in the AAA test group was 3.7 with a maximum score of 5 and minimum score of 2. The mean score per patient in the normal test group was 1.6 with a maximum score of 5 and minimum score of 0 .

In Figure $4.31 \mathrm{c}$ the threshold score of $\geq 2$ for a positive AAA diagnosis the AAA detection program correctly 'identified' $100 \%$ ( 7 out of 7 ) of the AAA test patients as having an $\mathrm{AAA}$, and $63.6 \%$ ( 7 out of 11 ) of the normal test patients as not having an AAA. The $100 \%$ sensitivity to AAA in the test group is excellent and the specificity 
level of $63.6 \%$ is acceptable given the limited size of the test sets. These results indicate that the objective analysis of CFA waveforms using the AAA detection program has potential as an alternative method for AAA detection.

\subsection{AAA Detection Program Performance for Patients with AAA and Additional}

\section{Aneurysmal Disease}

There were 9 patients within the AAA group with no significant atherosclerotic arterial disease that had aneurysmal disease of the iliac arteries or popliteal arteries in addition to an AAA. These 9 patients were not included in the study group used to design the MATLAB AAA detection program so that the effect of AAA alone could be investigated. The waveforms from these 9 patients were in effect a second 'blind' test set and were also subjected to the AAA detection program to test its response.

A summary of the AAA and additional aneurysm locations and diameters is given in Table A1.3, Appendix 1. It is worth noting that patients number 1 and 6 within this group had additional aneurysms of the popliteal artery and patient 3 had additional aneurysmal disease of the right CIA but the left CIA was normal calibre. All other patients within this group had bilateral iliac aneurysms in addition to an AAA.

The 6 indices calculated by the program are plotted for the left and right CFA waveforms for the 9 patients in the additional aneurysm group in Figures $4.32 \mathrm{a}$ to 4.32f. The threshold levels set by the AAA detection program for each of the indices are indicated by the dotted line on each graph. 


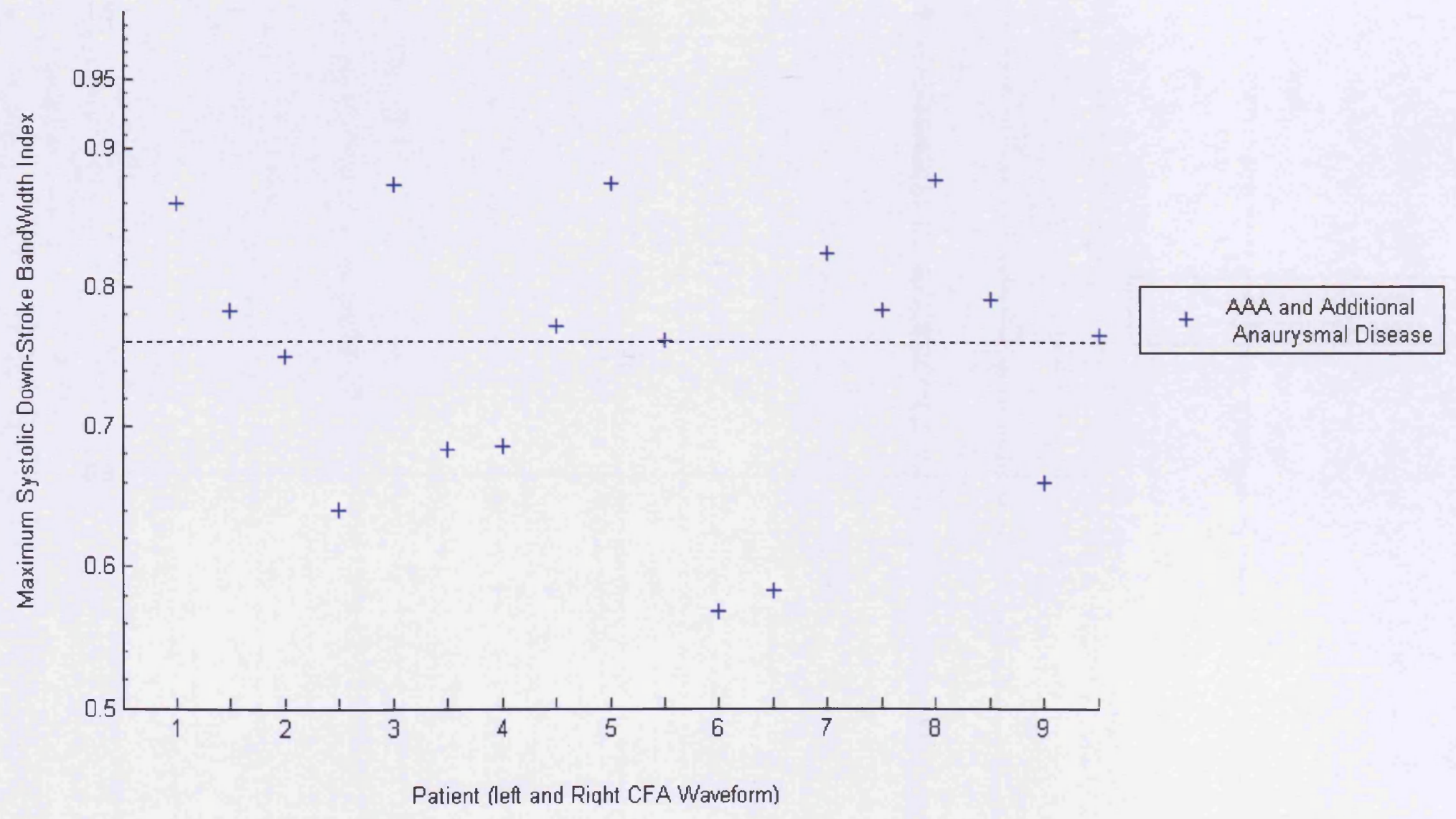

Figure 4.32a: Maximum Systolic Down-Stroke Bandwidth Index plotted for the left and right CFA waveforms for the 9 AAA patients with additional aneurysmal disease but no significant atherosclerotic disease. 


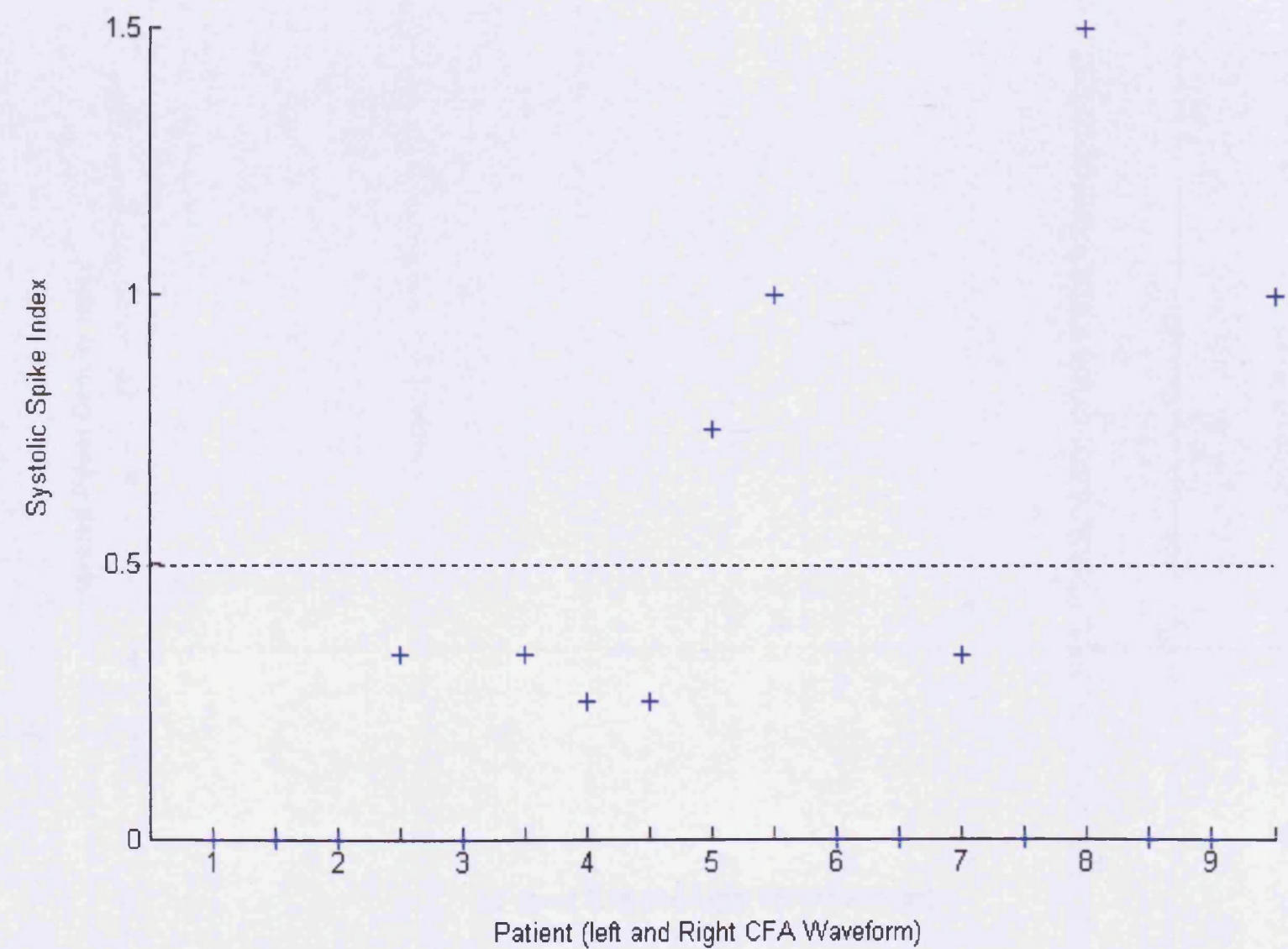

Figure 4.32b: Systolic Spike Index plotted for the left and right CFA waveforms for the 9 AAA patients with additional aneurysmal disease but no significant atherosclerotic disease. 


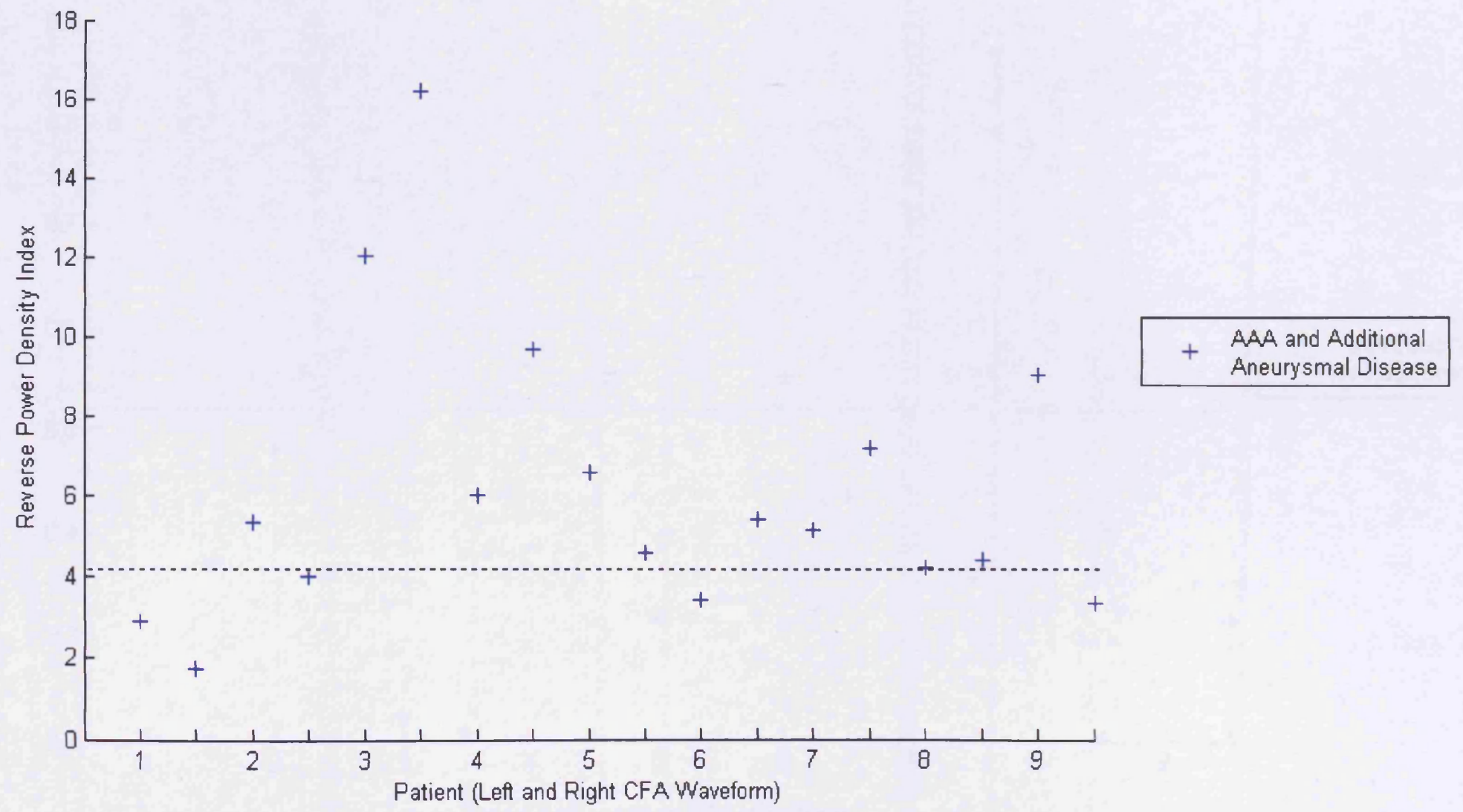

Figure 4.32c: Reverse Power Density Index plotted for the left and right CFA waveforms for the 9 AAA patients with additional aneurysmal disease but no significant atherosclerotic disease. 


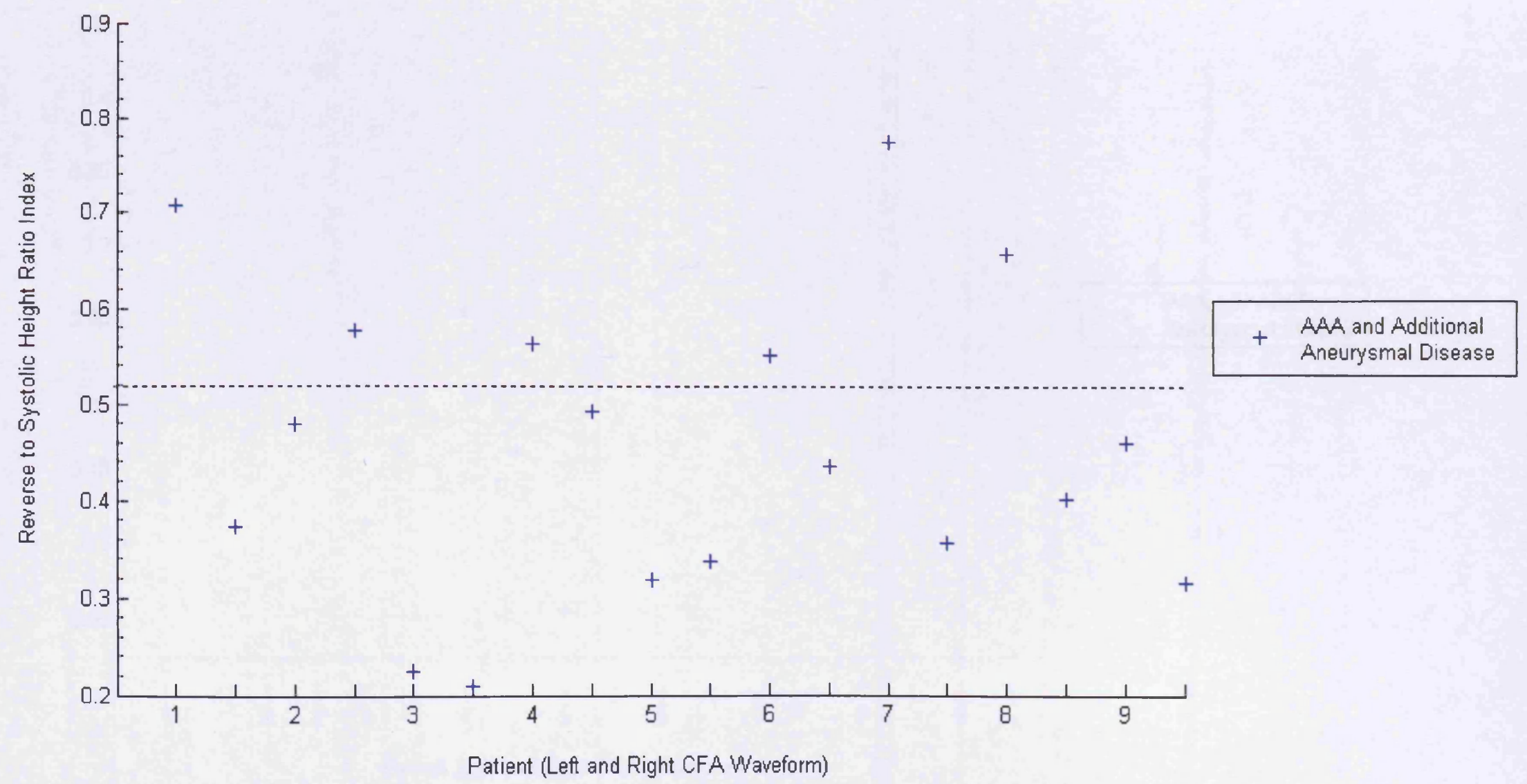

Figure 4.32d: Reverse to Systolic Height Ratio Index plotted for the left and right CFA waveforms for the 9 AAA patients with additional aneurysmal disease but no significant atherosclerotic disease. 


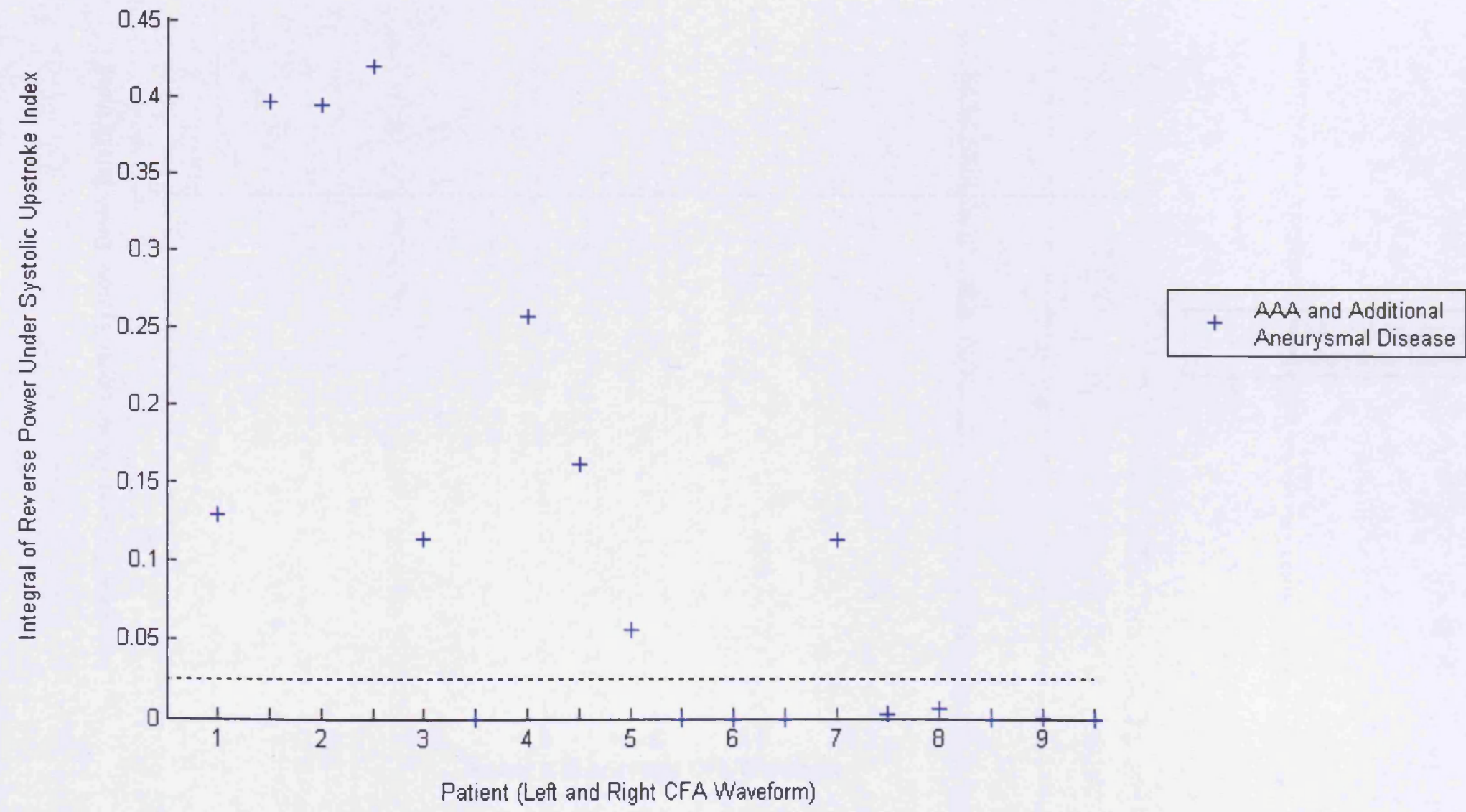

Figure 4.32e: Integral of Reverse Power Under the Systolic Upstroke Index plotted for the left and right CFA waveforms for the 9 AAA patients with additional aneurysmal disease but no significant atherosclerotic disease. 


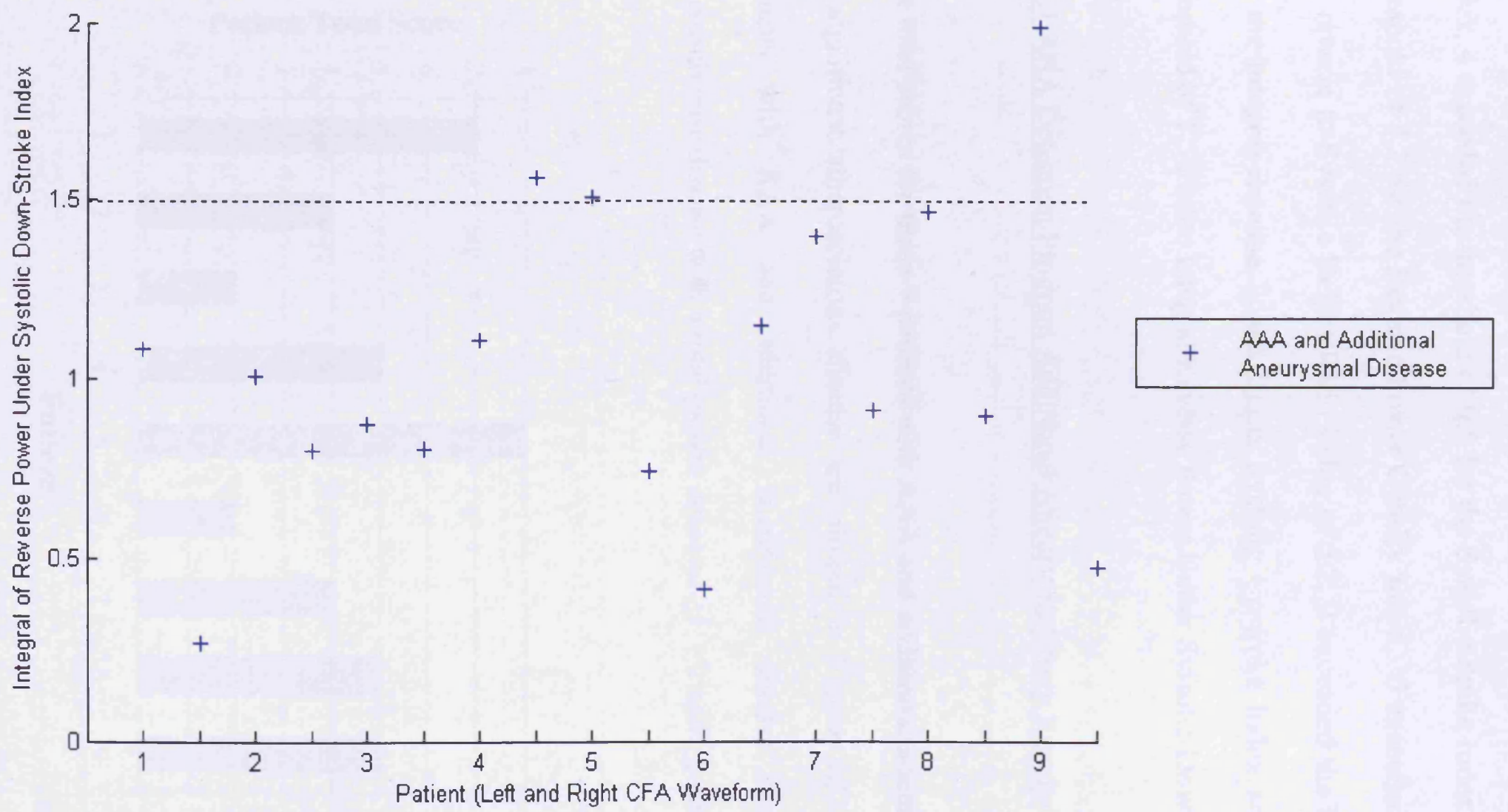

Figure 4.32f: Integral of Reverse Power Under the Systolic Down-Stroke Index plotted for the left and right CFA waveforms for the 9 AAA patients with additional aneurysmal disease but no significant atherosclerotic disease. 
Out of the 18 waveforms from patients with AAA and additional aneurysmal disease, 11 exceeded the threshold of 0.76 for the Maximum Systolic Down-Stroke Bandwidth Index, 4 exceeded the threshold of 0.5 for the Systolic Spike Index, 13 lay below the threshold of 4.2 for the Reverse Power Density Index, 6 exceeded the threshold for the reverse to Systolic Height Ratio Index of 5.2, 9 exceeded the threshold of 0.025 for the Integral Reverse Power Under Systolic Upstroke Index and 3 exceeded the threshold of 1.5 for the Integral Reverse Power Under Systolic Down-Stroke index.

\subsubsection{AAA Detection Program Additional Aneurysm Group Results}

The total scores for these 9 patients with AAA and additional aneurysmal disease but no significant atherosclerotic disease are plotted in Figure 4.33. The number of patients with AAA and additional aneurysmal disease but no significant atherosclerotic disease with a total patient score of $\geq 1, \geq 2$ and $\geq 3$ are plotted in Figure 4.34 .

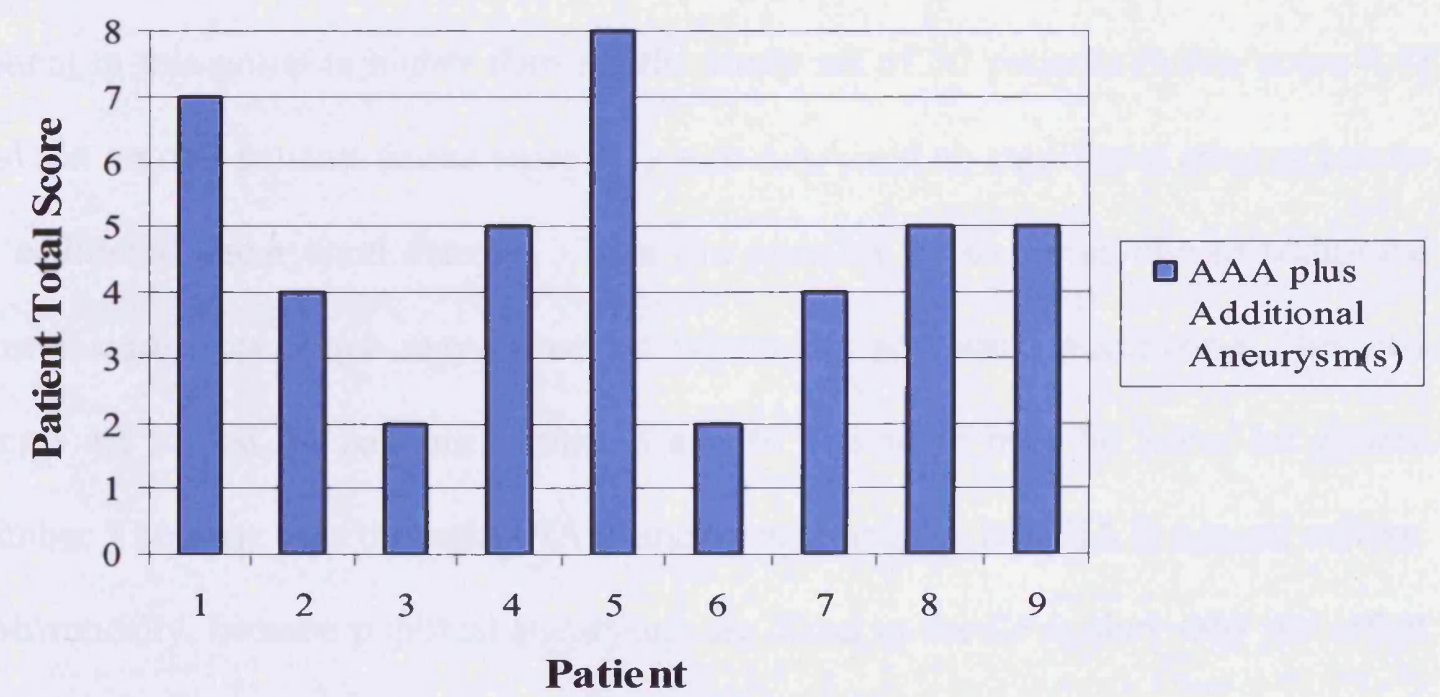

Figure 4.33: Total Patient Scores for the 9 AAA patients in with additional aneurysmal disease but no significant atherosclerotic disease. 


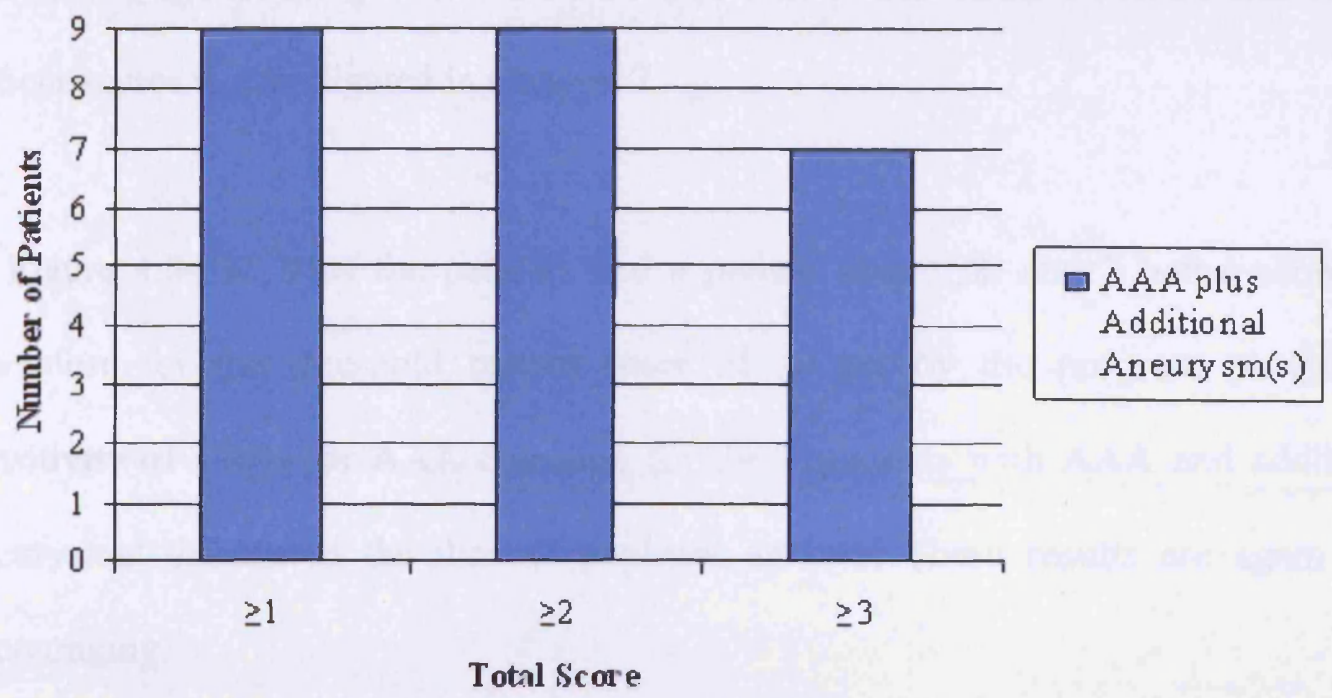

Figure 4.34: Number of AAA patients with additional aneurysmal disease but no significant atherosclerotic disease with total patient scores of $\geq 1, \geq 2$ and $\geq 3$.

\subsubsection{AAA Detection Program Additional Aneurysm Group Results: Discussion}

It can be seen from Figure 4.33 that the mean score per patient in this patient group was 5.3 with a maximum score of 8 and minimum score of 2 . The mean score per patient in this group is higher than for the study set of 30 patients (mean score 4.8) and test set of 7 patients (mean score 3.7) with AAA and no significant atherosclerotic or additional aneurysmal disease. This can possibly be expected due to additional flow disturbances which may be set up within the additional aneurysms. The total scores are lowest for patients number 3 and 6 . The score may be lower for patient number 3 because only the right CIA is aneurysmal and the left CIA is normal calibre. Theoretically, because popliteal aneurysms are distal to the CFA, they may not affect the CFA waveform to the same extent as a proximal iliac aneurysm. The score for patients numbers 1 and 6 with AAA and popliteal aneurysms were 7 and 2 respectively. The score may possibly be higher for patient 1 because the AAA for this 
patient was $5.7 \mathrm{~cm}$ compared to $3.5 \mathrm{~cm}$ for patient 6 . The effect of AAA size on the patient scores is investigated in Chapter 7.

In Figure 4.34 all 9 of the patients had a patient score $\geq 2$, and 7 had a score $\geq 3$. Therefore for the threshold patient score of $\geq 2$ set by the program provided a sensitivity of $100 \%$ for AAA detection for the 9 patients with AAA and additional aneurysmal disease of the iliac or popliteal arteries. These results are again very encouraging. 


\subsection{Discussion}

At the beginning of this chapter, MATLAB code was written to calculate parameters from which indices to describe the visual features noted in the CFA waveforms in Chapter 3 could be derived. There were 6 indices chosen to represent the 5 features:

1) The Maximum Systolic Down-Stroke Bandwidth Index to measure the feature of increased spectral broadening on the systolic down-stroke, 2) The Systolic Spike Index to measure the presence of spikes on the systolic down-stroke, 3) The Reverse to Systolic Height Ratio Index to measure the feature of an irregular reverse flow pattern, 4) The Reverse Power Density Index to provide a measure of both the feature of irregular reverse flow and the feature of waveform elongation, and 5) The Integral Reverse Power Under the Systolic Upstroke Index and the Integral Reverse Power Under the Systolic Down-Stroke Index to measure the feature of simultaneous forward and reverse flow. These 6 indices, along with the corresponding threshold levels and weighting factors given in Table 4.12, and with a threshold of $\geq 2$ applied to the total patient score achieved a very good level of discrimination between AAA and normal patients without significant atherosclerotic disease. The $100 \%$ sensitivity and $73.3 \%$ specificity achieved by the AAA detection program for the 30 AAA and 30 normal patients in the study groups with no significant atherosclerotic arterial disease improved on the results achieved for these patients in the visual analysis of the waveforms of $93.3 \%$ sensitivity $70 \%$ specificity. The response of the AAA detection program to the blind test groups containing 7 AAA and 11 normal patients was encouraging given the limited sizes of the test groups. The sensitivity of $100 \%$ and specificity of $63.6 \%$ were in line with the levels achieved from the 30 AAA and 30 normal patients in the study groups used to select the indices, thresholds and 
weighting factors in the program. The programs response of $100 \%$ sensitivity to the group of 9 AAA patients with additional aneurysmal disease which also acted as a second blind test group was also very promising.

The overall response of the AAA detection program for the 46 patients with AAA and no significant atherosclerotic arterial disease (the study group, plus the test group, plus the additional aneurysmal group patients) and 41 patients without AAA and no significant atherosclerotic disease (the study group plus the test group patients) was a sensitivity of $100 \%$ for AAA detection (46 out of 46 correctly identified as having AAA), and a specificity of $70.7 \%$ (29 out of 41 normal patients correctly identified as not having AAA). If these results are projected onto to the general population with no significant aorto-iliac or femoro-popliteal atherosclerotic arterial disease, assuming the prevalence of lower limb PAD is around $12 \%$, this gives a sensitivity of around $100 \%$ and a specificity of around $70 \%$ for approximately $88 \%$ of the general population. These levels indicate that this type of objective analysis of the CFA waveform has the possible potential for use as an alternative method to B-mode ultrasound for AAA detection.

The screening studies for AAA discussed in Chapter 1 indicate that a single B-mode scan would be performed on all males of 65 years old. PAD is a progressive disease and the studies indicate that the prevalence of PAD is around 7\% for those aged $60-69$ years old, and increases to around $23 \%$ for those aged over 80 years old (Fowkes, Housley et al. 1991; Ostchega, Paulose-Ram et al. 2007). Therefore if the performance of this AAA detection program is projected onto the potential screened population, assuming $93 \%$ of the screened population would not have significant 
atherosclerotic arterial disease, the AAA detection program would provide a sensitivity of around $100 \%$ and specificity of around $70 \%$ for approximately $93 \%$ of the screened population.

In order to determine the feasibility of using these CFA analysis methods to detect AAA in the remaining $7 \%$ of the screened population with significant atherosclerotic disease, the visual feature analysis of the CFA waveforms was applied to the patient groups with significant atherosclerotic disease of the aorto-iliac or femoro-popliteal arteries. The objective MATLAB based AAA detection technique was then applied to these significant atherosclerotic disease groups. The applications of the visual and objective feature analysis of the CFA waveforms to the significant atherosclerotic disease groups are discussed in Chapters 5 and 6 respectively. 
Chapter 5: Visual Inspection of CFA Waveforms from AAA and No AAA

Patients with Significant Atherosclerotic Disease

\subsection{Introduction and Aims}

In Chapter 3, visual examination of CFA waveforms from 30 patients with AAA and 30 patients without AAA with no significant aorto-iliac or femoro-popliteal atherosclerotic arterial disease identified 5 features that differed from an 'ideal' CFA waveform that were more prominent in the group of AAA patients: 1) spectral broadening on the systolic down-stroke, 2) the appearance of spikes on the systolic down-stroke, 3) an irregular reverse flow pattern, 4) simultaneous forward and reverse flow during systole and 5) elongation of the waveform during the cardiac cycle. Based on the visual identification of these features it was possible to detect AAA in these patients with a sensitivity of $93.3 \%$ and a specificity of $70 \%$. In this chapter, waveforms from the patients with and without AAA but with significant atherosclerotic disease of the iliac and/or femoro-popliteal arteries were visually assessed to determine whether these 5 features and whether any additional features were present. The results were used to determine whether the visual waveform analysis method could also be used to detect AAA in patients with significant atherosclerotic disease of the aorto-iliac and/or femoro-popliteal arteries. 


\subsection{Method}

The cohort for the first part of this study comprised the study groups containing the 15 AAA and 18 no AAA patients with significant atherosclerotic femoro-popliteal (distal) arterial disease (summarised in Table A3.1, Appendix 3 and Table A4.1, Appendix 4). The cohort for the second part of this study comprised the study groups containing the 6 AAA and 7 no AAA patients with significant iliac (proximal) disease (summarised in Table A3.3, Appendix 3 and A4.3, Appendix 4). The patients in the significant distal disease groups had at least one significant $(>50 \%)$ stenosis or occlusion of the femoro-popliteal arteries. 3 patients in each group had unilateral disease. These were patients number 2, 10 and 11 in the AAA group and patients numbers 5,11 and 18 in the no AAA group, all of whom had significant disease in the left leg and diffuse disease but no significant femoro-popliteal focal lesions in the right leg. The remaining patients had significant distal disease bilaterally. All patients in the significant proximal disease groups had at least one significant stenosis $(>50 \%)$ or occlusion of the iliac arteries. 2 patients in the AAA group and 3 patients in the no AAA group had bilateral proximal disease. Patients number 2,3 , and 5 in the AAA group had significant proximal disease on the left side only. Patient number 6 had an occlusion of the left CFA and therefore a CFA waveform was not measured, and in addition to an AAA the right CIA was also aneurysmal. Patient numbers 2 and 4 had no significant distal disease bilaterally. Patients number 1,3, 4 and 6 had bilateral distal disease in addition to significant proximal disease. In the no AAA patient group, patients number 1, 4 and 6 had significant proximal disease on the left side only and patient number 3 had significant proximal disease on the right side only. In addition to significant proximal disease, patients number 2,4 , and 5 had bilateral 
significant distal disease. Patients number 1, 3, 6 and 7 had no significant distal disease.

The distal and proximal disease groups were analysed separately. As in Chapter 3, the CSV data files for the left and right CFA waveforms for each patient were reproduced as spectrograms in MATLAB using the 'imagesc' plotting function in the 'vga' colour scale. The waveform cycles within the CFA spectrograms were visually inspected for the 5 features: 1) spectral broadening on the systolic down stroke, 2) the appearance of spikes on the systolic down-stroke, 3) an irregular reverse flow pattern, 4) simultaneous forward and reverse flow during systole and 5) elongation of the waveform during the cardiac cycle, and also to determine whether any additional features were present in the waveforms.

\section{$\underline{5.3 \text { Results }}$}

\subsubsection{Visual Inspection of CFA Waveforms from Patients with Significant Femoro-}

\section{Popliteal Disease}

\subsubsection{Feature Results}

As would be expected, the majority of the CFA waveforms in both the AAA and no AAA significant distal disease groups were 'higher impedance' due to the presence of the atherosclerotic disease. Just over half the waveforms in both groups were biphasic rather than triphasic. There were features in the waveforms characteristic of distal disease such as a reduced reverse flow component or a shoulder on the systolic 
down-stroke, but there were no additional features displayed in the waveforms that were more prominent in the AAA group that were different from the 5 features noted in Chapter 3. Therefore the waveforms were individually scored according to the presence of these 5 features with a score of 1 awarded if the feature was present in one or more cycles within the waveform. Figure 5.1 shows the number of CFA waveforms from the AAA and no AAA patient groups with significant femoropopliteal (distal) disease with each feature present. Again, as for the no significant atherosclerotic disease groups, in general the combination of features present in the left and right CFA waveforms for the same patient were not always the same.

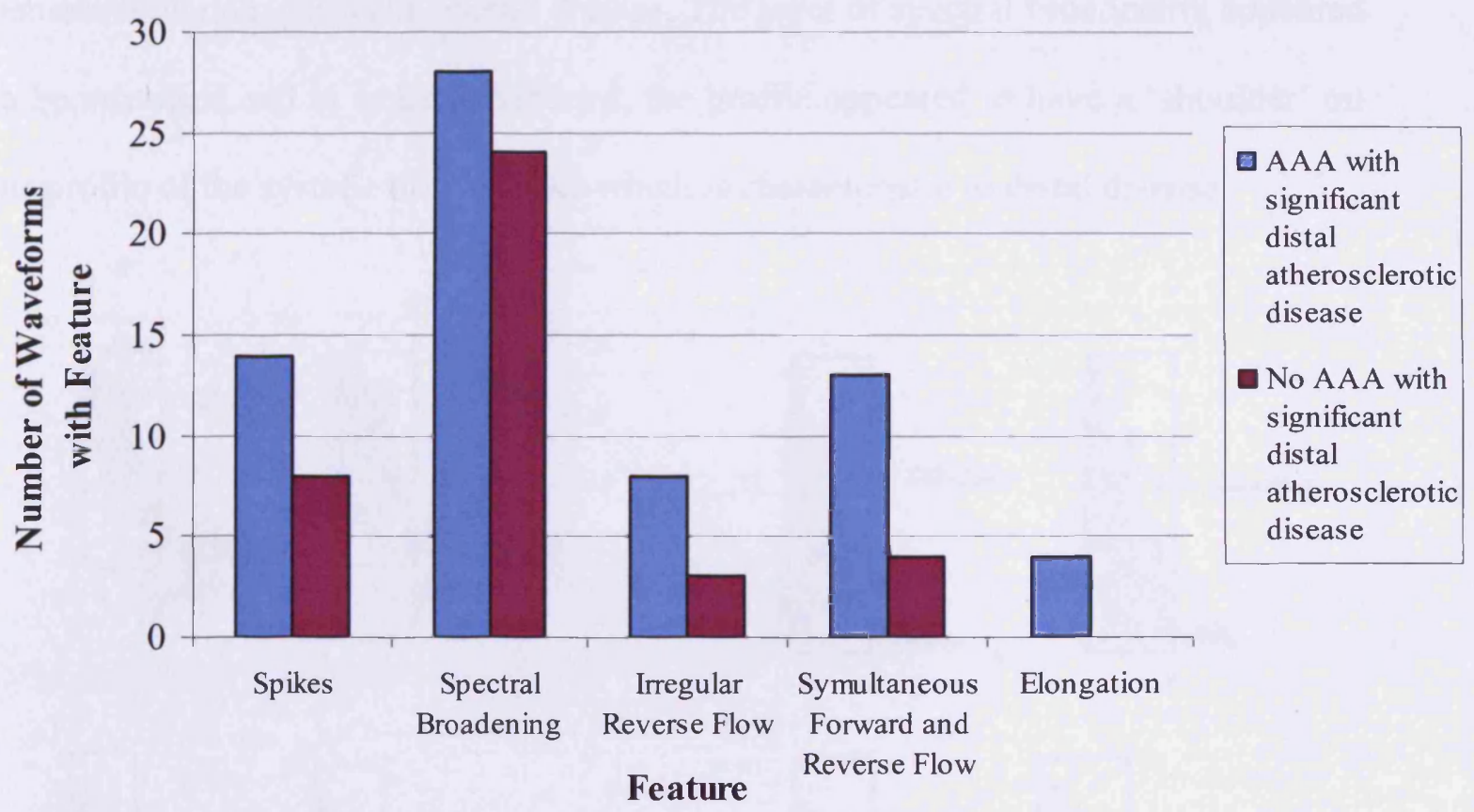

Figure 5.1: Number of CFA waveforms out of the 30 AAA and 36 no AAA CFA waveforms in the significant distal arterial disease study groups with each feature present visually 


\subsubsection{Description of Features}

The feature of spectral broadening was present in 28 out of the 30 AAA waveforms and 24 out of the 36 no AAA waveforms. This is a much higher proportion in both the AAA and no AAA groups than for the waveforms in the no significant atherosclerotic disease groups in Chapter 3 (for which 37 out of the 60 AAA waveforms and 14 out of the 60 normal waveforms displayed increased spectral broadening). Examples of the waveform cycles from AAA and no AAA patients with significant distal disease displaying increased spectral broadening are shown in Figure 5.2 and Figure 5.3 respectively. In some cases, the pattern of spectral broadening in the waveforms in patients with distal disease was different to that observed in the waveforms from patients with no significant arterial disease. The level of spectral broadening appeared to be increased and in some waveforms, the profile appeared to have a 'shoulder' on the profile of the systolic down-stroke which is characteristic to distal disease.

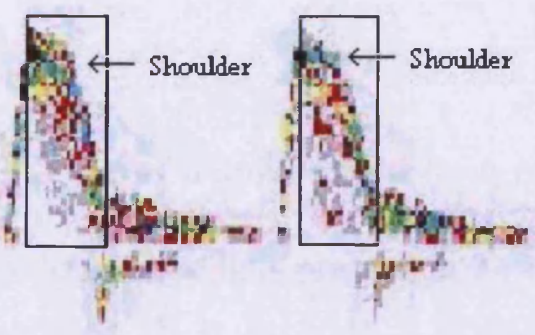

a)

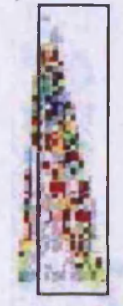

c)

Figures 5.2a-d: Examples of spectral broadening on the systolic down-stroke in AAA patients with significant femoro-popliteal atherosclerotic arterial disease

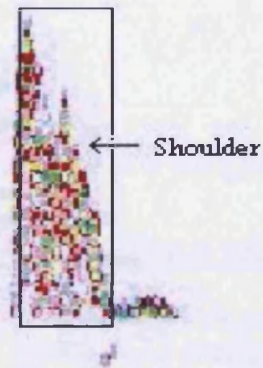

b)

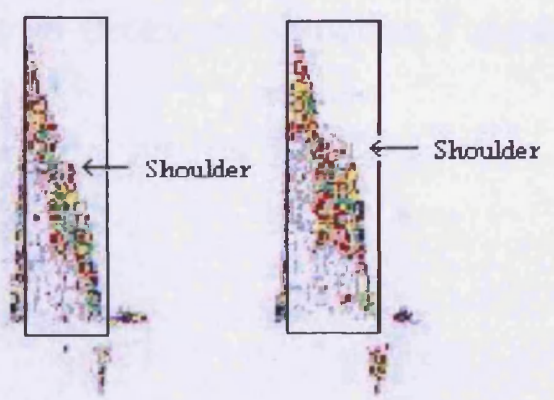

)

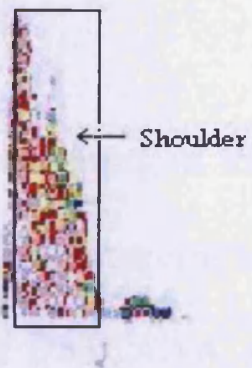
179 


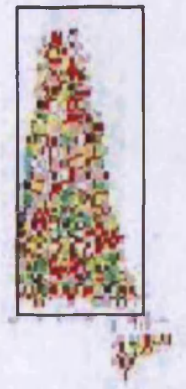

a)

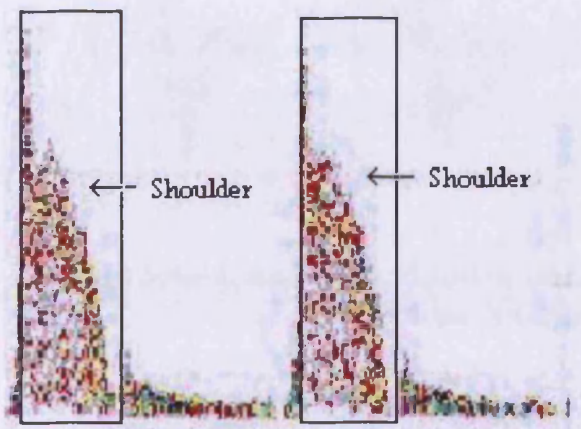

c)

Figures 5.3a-d: Examples of spectral broadening on the systolic down-stroke in no AAA patients with significant femoro-popliteal atherosclerotic arterial disease

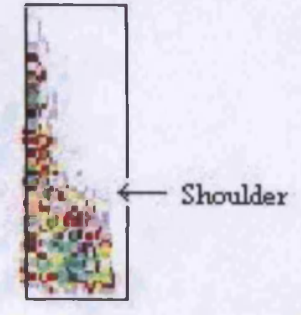

*

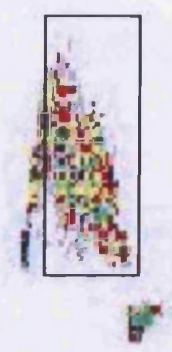

d)

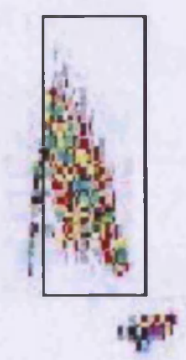

(1)

The feature of a spiked profile in the spectral broadening in the downward slope of the systolic peak was present in 14 out of 30 AAA waveforms and 8 out of the 36 no AAA waveforms. Examples of waveform cycles from AAA and no AAA patients displaying the feature of spikes on the systolic down-stroke are shown in Figures 5.4 and 5.5 respectively. 


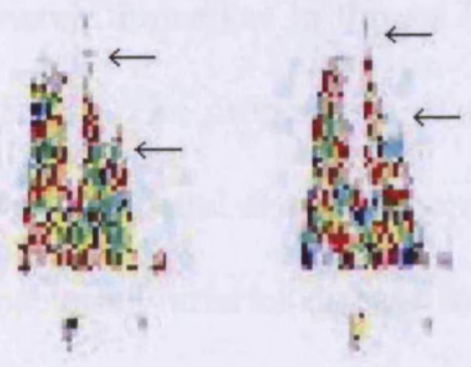

a)

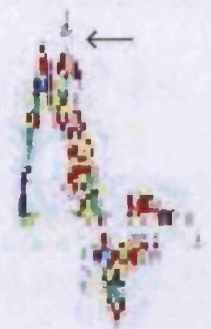

c)
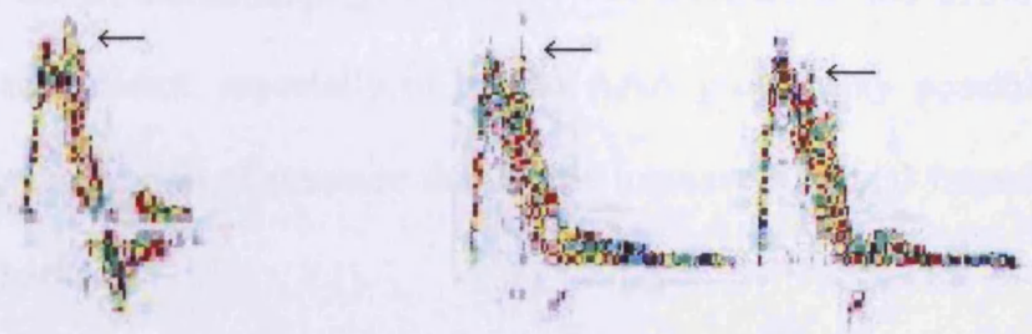

d)

b)

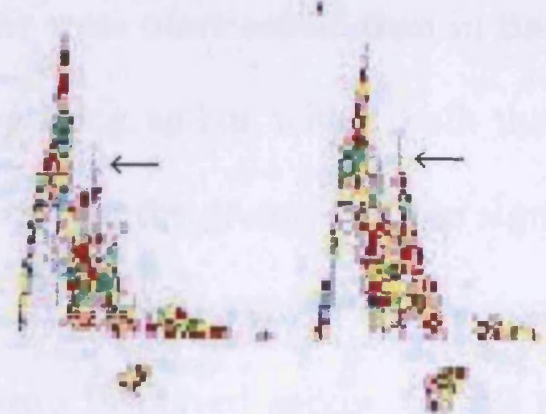

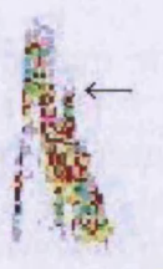

whe

a)

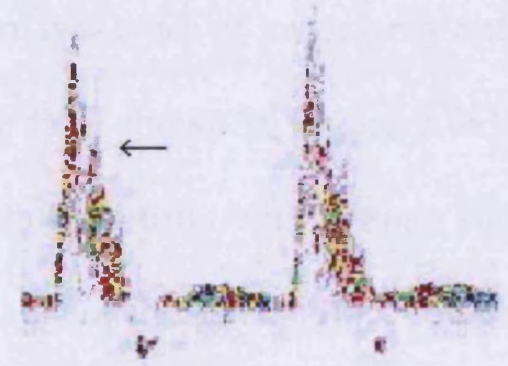

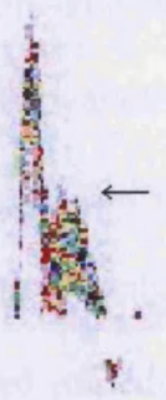

b)

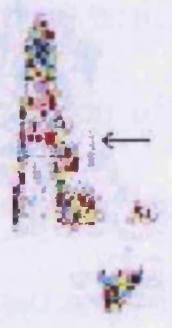

d)

c)

Figures 5.5a-d: Examples of spikes on the systolic down-stroke in no AAA patients with significant femoro-popliteal atherosclerotic arterial disease
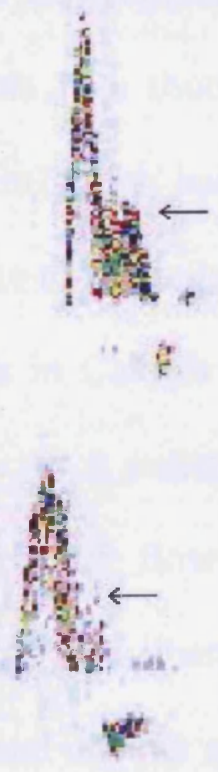
In general, the spikes in the no AAA waveforms were more subtle than in the AAA waveforms. The proportions of waveforms displaying spikes within both the AAA and no AAA distal disease groups were higher than for the groups with no significant atherosclerotic arterial disease in Chapter 3 (for the AAA group with no significant atherosclerotic disease 23 out of the 60 waveforms displayed spikes, for the normal patient group only 3 out of the 60 displayed spikes). The increase in the number of waveforms with spikes present, especially in the no AAA group may possibly be attributed to increased reflection of pressure due to the increase in distal impedance caused by the distal disease.

In general, due to the atherosclerotic disease, the reverse flow phases within the waveforms were reduced in both the AAA and no AAA groups, possibly due to the increased impedance to flow. This resulted in a 'stumped' appearance to the reverse flow phase as illustrated in Figures 5.3a and 5.3b. The feature of an irregular reverse flow pattern was present in 8 of the 30 AAA waveforms, this is a much lower proportion than in the AAA group with no significant disease in which half of the AAA waveforms displayed this feature. This may possibly be due to the reduction of the reverse flow component due to the increased impedance. As in Chapter 3 , there were two types of irregularity noted in the reverse flow within the AAA patient group. The first, shown in Figure 5.6a illustrates an elongation of the reverse flow with an irregular profile with distinct spikes, the second, shown in Figure 5.6b illustrates an 'exaggerated' reverse flow component with increased amplitude and distinct spikes. 
a)

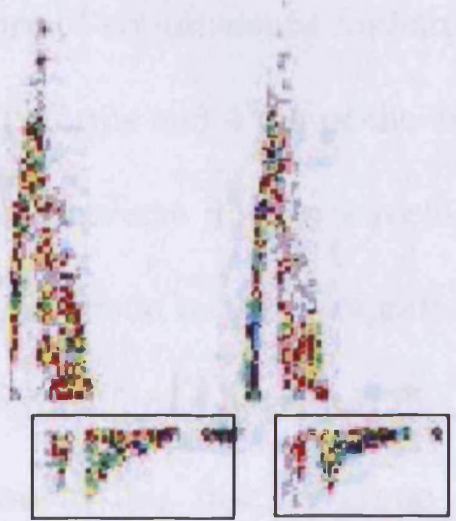

Figure $5.6 \mathrm{a}$ and $\mathrm{b}$ : Examples of irregular reverse flow patterns for AAA patients with significant

femoro-popliteal atherosclerotic arterial disease

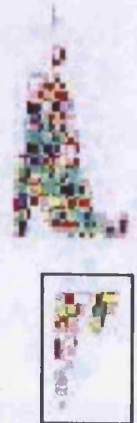

b)

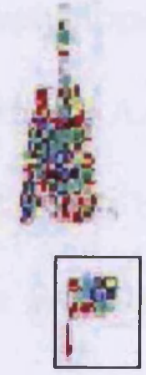

The irregular reverse flow pattern was present in 3 out of the 36 no AAA waveforms which is a similar proportion to that in the no AAA no significant atherosclerotic disease group. The irregularities were less distinct in these no AAA waveforms than in the AAA waveforms. An example of waveform cycles from a no AAA patient displaying irregular reverse flow is given in Figure 5.7.
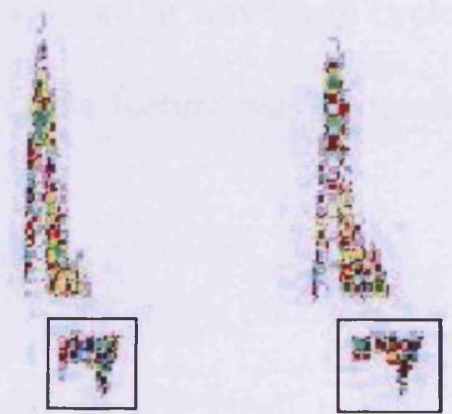

Figure 5.7: Example of irregular reverse flow pattern for no AAA patient with significant femoropopliteal atherosclerotic arterial disease 
The feature of simultaneous forward and reverse flow was present in 13 out of the 30 AAA waveforms and 4 out of the 36 no AAA waveforms. Examples of simultaneous forward and reverse flow in waveform cycles from AAA patients are given in Figure 5.8. In comparison to the no significant atherosclerotic disease group in which 21 out of the 60 waveforms displayed this feature, the proportion of AAA waveforms with the feature in this disease group is slightly higher. The proportion of no AAA waveforms displaying this feature is also increased as only 1 out of the 60 waveforms in the no significant atherosclerotic disease group displayed simultaneous forward and reverse flow.

In this feature, low power reverse flow can be seen under the systolic peak, before the onset of the 'true' reverse flow component. This can possibly be attributed to flow disturbances occurring during the systolic flow phase. In Chapter 3, for patients with no significant atherosclerotic arterial disease, the flow disturbances could possibly be attributed to the presence of AAA. However the presence of atherosclerotic disease also disturbs the flow and this could explain why this feature is more prevalent in both the AAA and no AAA distal disease groups than in the no significant disease groups. Examples of this feature displayed in waveform cycles from a patient without AAA is given in Figure 5.9. Again, this feature was generally more pronounced in the AAA waveforms. 


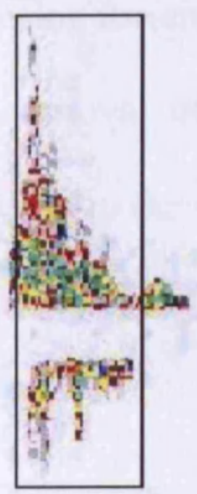

a)

Figure 5.8 a-b: Examples of simultaneous forward and reverse flow in AAA waveforms with significant femoro-popliteal atherosclerotic arterial disease
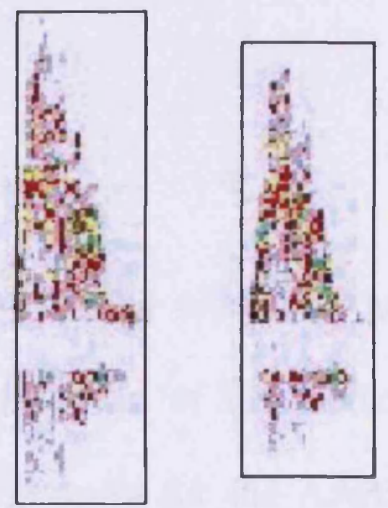

Figure 5.9: Example of simultaneous forward and reverse flow in a no AAA waveform with significant femoro-popliteal atherosclerotic arterial disease

The feature of waveform elongation was present in only 4 out of the 30 AAA waveforms and was not present in any of the no AAA waveforms. An example of the elongation in AAA waveform cycles is given in Figure 5.10. There may be several reasons why waveform elongation is less prevalent in the significant atherosclerotic disease patients: the effect of minor atherosclerotic disease of the proximal arteries 
reducing the amplitude of the flow at the CFA, the influence of distal impedance, and the removal of low amplitude reverse flow by the high pass 'wall thump' filter inherent in the system.

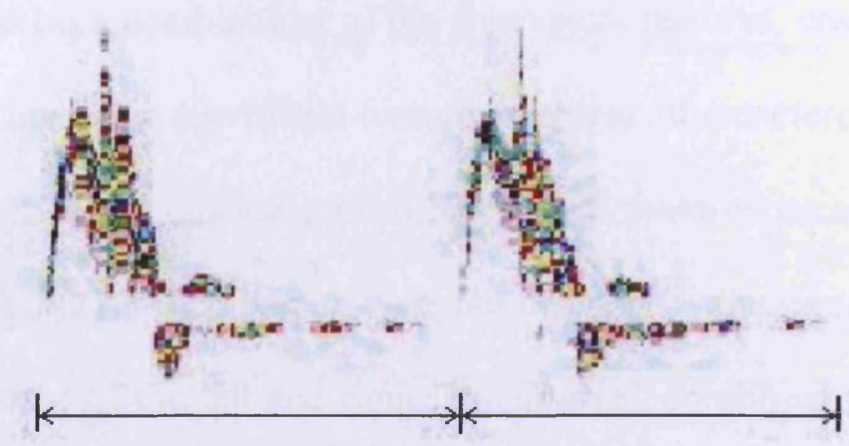

Figure 5.10: Example of Elongation in AAA waveform with significant femoro-popliteal atherosclerotic arterial disease

It can be seen in Figure 5.1, that the separation between the AAA and no AAA waveforms provided by identification of the 5 visual features is poorer than the separation provided by these features for the AAA and no AAA patients without significant atherosclerotic disease in Figure 3.12 (Chapter 3). The additional changes to the CFA waveforms caused by the presence of significant femoro-popliteal atherosclerotic disease, make it much more difficult to discriminate between CFA waveforms from patients with and without AAA by visual feature analysis. Each individual feature does show a level of discrimination, but there is no single feature that is characteristic to AAA. In the following section, the scores for the five waveform features were combined to determine whether AAA could be identified more successfully by detection based on the combination of the 5 visual features. 


\subsubsection{AAA Detection in Patients with Significant Femoro-Popliteal Atherosclerotic}

\section{Disease Using CFA Visual Feature Analysis}

The same method that was applied to the patients in the no significant atherosclerotic disease groups in Chapter 3 was used to determine whether visual analysis of the CFA waveforms, based on a combination of the five visual features, could correctly detect AAA in the patients in the significant femoro-popliteal atherosclerotic disease groups. As for the no significant disease groups, the visual features present in the left and right CFA waveforms for each patient were not necessarily the same. This may be due to the different paths of the left and right iliac arteries, combined with differences in the pattern of proximal and distal atherosclerotic disease between the two sides. Therefore for each patient, the total scores for the waveforms from the left and right legs were summed to give a total patient score.

The total scores for the $15 \mathrm{AAA}$ and 18 no AAA patients are plotted in Figures $5.11 \mathrm{a}$ and $5.11 \mathrm{~b}$. The mean factor score per patient in the AAA group was 4.5 with a maximum score of 8 factors and a minimum score of 2 factors. The mean total factor score per patient in the no AAA group was 2.2 with a maximum score of 6 factors minimum score 0 factors. In comparison with the results obtained from the no significant disease groups, the mean score for the AAA group is similar but the mean score in the no AAA group is increased. This may be expected due to the increase in flow disturbances caused by the atherosclerotic disease, making it more difficult to differentiate between the two groups. 


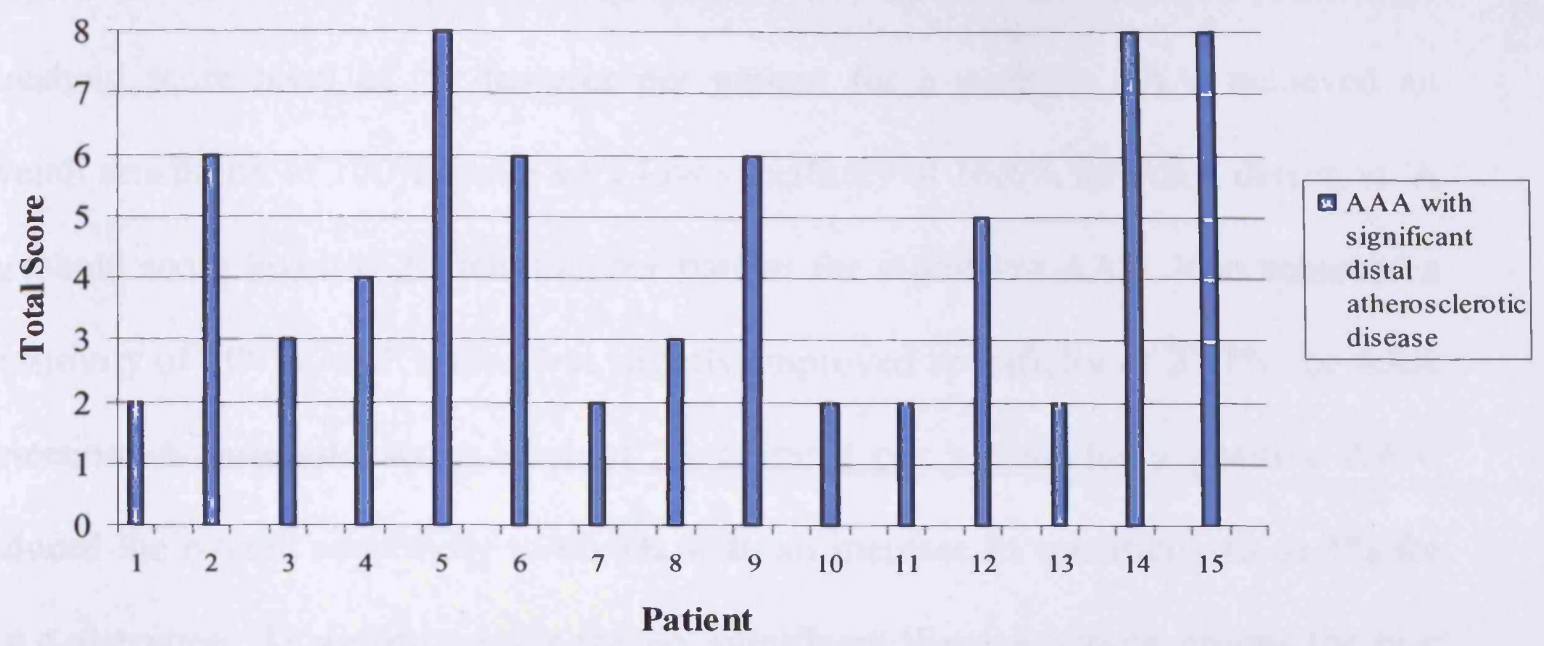

Figure 5.11a: Total Patient Scores for the 15 AAA patients with significant femoro-popliteal atherosclerotic disease.

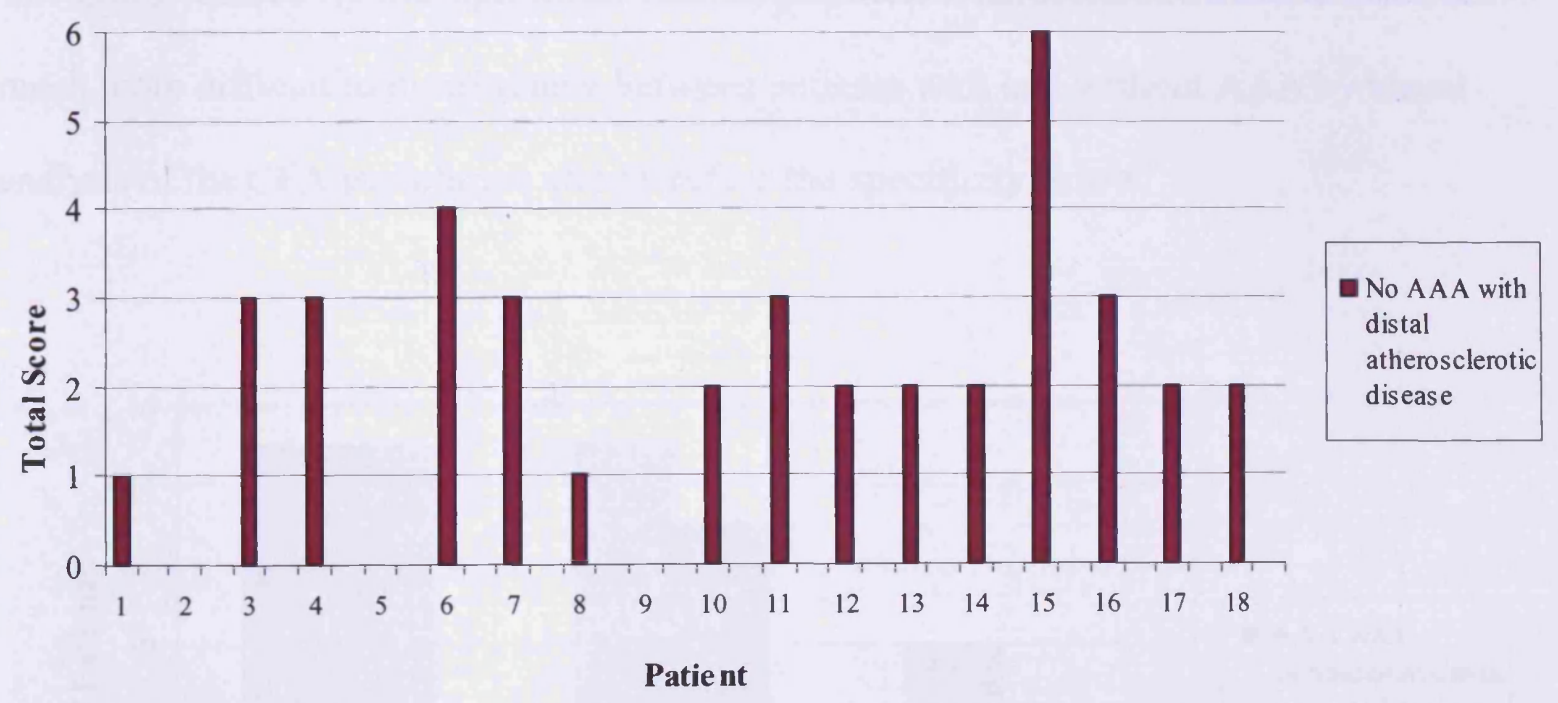

Figure 5.11b: Total Patient Scores for the 18 no AAA patients with significant femoro-popliteal atherosclerotic disease.

Following the method described in Chapter 3, the total patient scores were used to set a threshold level to best distinguish between the AAA and no AAA groups. Figure 5.12 shows the number of AAA and no AAA patients with significant atherosclerotic 
disease of the femoro-popliteal arteries with $\geq 1, \geq 2$ and $\geq 3$ features present. A threshold score level of $\geq 1$ features per patient for a positive AAA, achieved an overall sensitivity of $100 \%$, but a very low specificity of $16.6 \%$ for AAA detection. A threshold score level of $\geq 2$ features per patient for a positive AAA, also achieved a sensitivity of $100 \%$, with a low, but slightly improved specificity of $27.7 \%$ for AAA detection. A threshold score level of $\geq 3$ features per patient for a positive AAA, reduced the overall sensitivity to $66.6 \%$ with an increase in specificity to $61.1 \%$ for AAA detection. Therefore, as for the no significant disease patient groups the best threshold for AAA detection was a patient score of $\geq 2$ as this provided $100 \%$ sensitivity with the higher level of specificity. The additional changes to the CFA waveforms caused by the significant femoro-popliteal atherosclerotic disease made it much more difficult to discriminate between patients with and without AAA by visual analysis of the CFA waveforms and therefore the specificity is low.

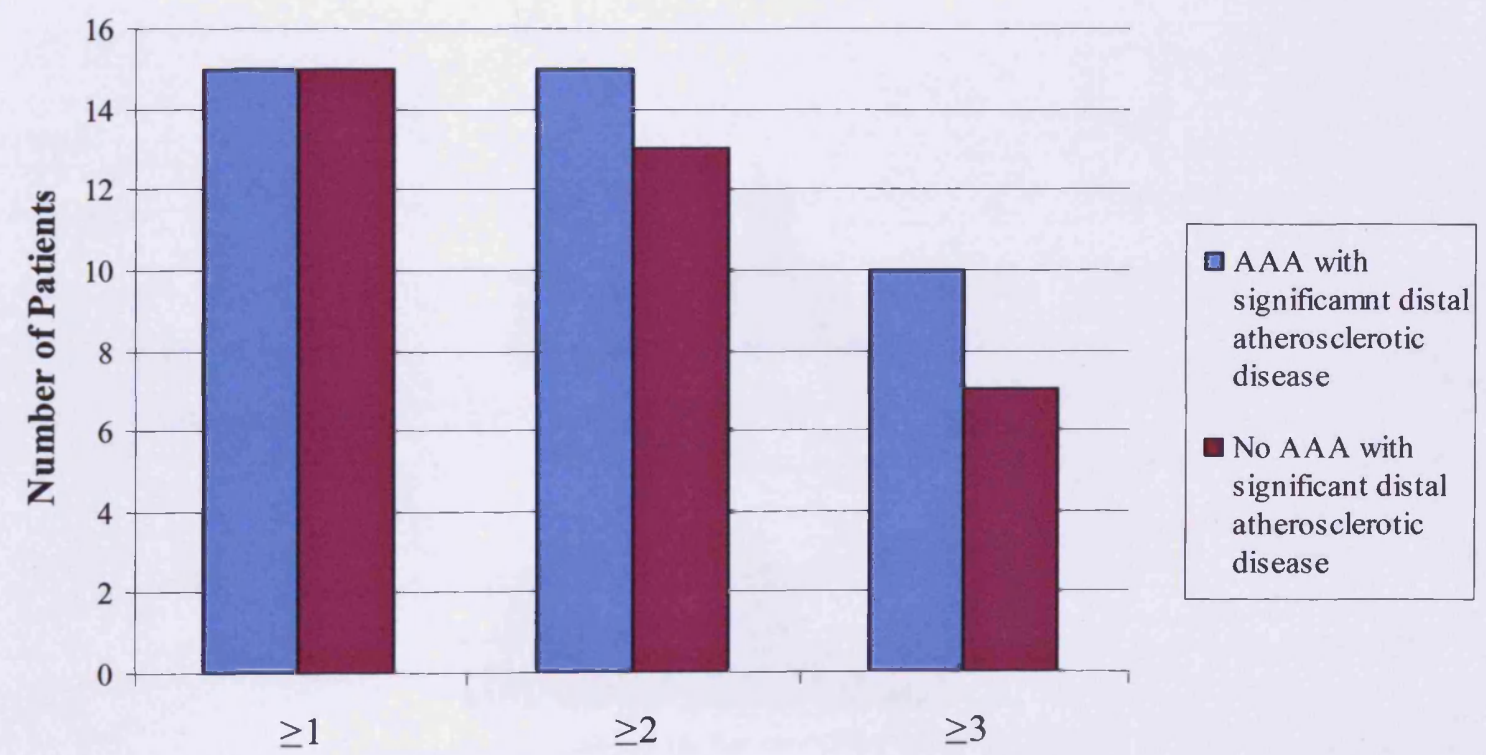

Total Score

Figure 5.12: Number of AAA $(n=15)$ and no AAA $(n=18)$ patients in the study groups with significant femoeo-popliteal atherosclerotic disease with total score $\geq 1, \geq 2$ and $\geq 3$ 
5.3.2 Visual Inspection of CFA Waveforms from Patients with Significant Proximal $\underline{\text { Atherosclerotic Disease }}$

\subsubsection{Feature Identification}

Proximal disease was present for 8 out of the 11 AAA waveforms and 10 out of the 14 no AAA waveforms from the 6 AAA and 7 no AAA patients in the significant proximal disease study groups. On visual inspection, all the CFA waveforms from the limbs with significant iliac disease were damped. 7 out of the 8 waveforms from patients with AAA, and 9 out of the 10 waveforms from patients without AAA were 'mono-phasic'. Examples of these mono-phasic waveform cycles are given in Figure 5.13. It can be seen that there was no real differences between the profiles of the AAA and no AAA mono-phasic waveforms.

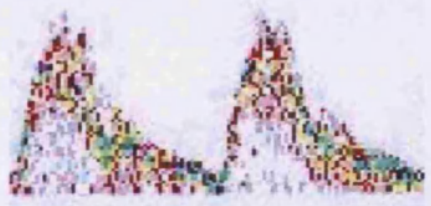

a)

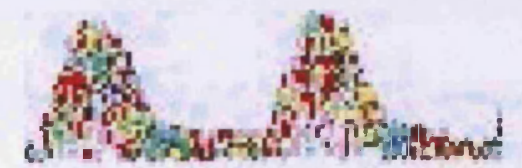

b)

Figure 5.13: Damped 'mono-phasic' CFA waveform with significant proximal disease and a) AAA and b) No AAA. 
All the waveforms in both groups with proximal disease displayed the feature of spectral broadening. 2 out of the 8 AAA waveforms and 3 out of the 10 no AAA waveforms had the appearance of spikes on the down-stroke of the peak of the waveform cycle as illustrated in Figure 5.14. However, it is probably not possible for flow disturbances set up within an AAA to be transmitted through a tight iliac artery stenosis or through collateral arteries in the case of an iliac occlusion. Therefore, these features are more likely to be due to flow disturbances set up by proximal stenotic, local or distal disease rather than by an AAA.

a)
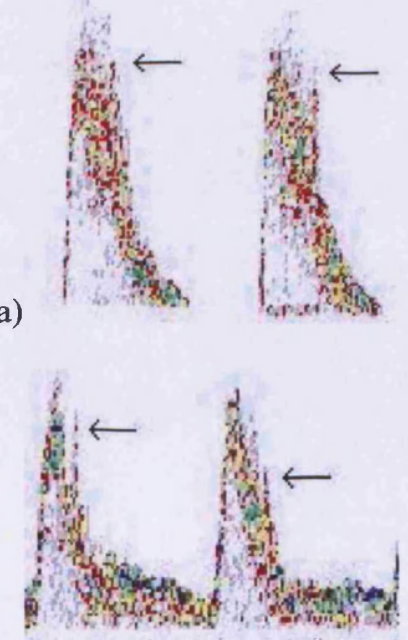

b)

Figure 5.14: Spikes on peak down-stroke of damped waveform cycles for patients with significant proximal disease and a) AAA and b) No AAA

The feature of irregular reverse flow, as illustrated in Figure 5.15, was only present in 2 out of the 8 AAA waveforms and was not exhibited in any of the 10 no AAA waveforms. The feature of simultaneous forward and reverse flow was present in 3 of the AAA waveforms and only 1 of the no AAA waveforms. This feature is illustrated for waveform cycles from AAA and no AAA patients in Figure 5.16 and is also illustrated in the waveform cycles from the AAA patient in Figure 5.15. None of the CFA waveforms in the proximal disease groups exhibited the feature of elongation. 


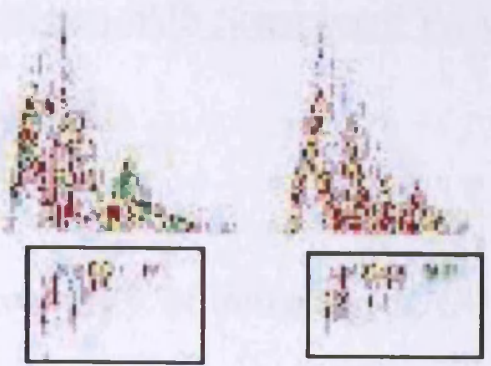

Figure 5.15: Irregular reverse flow pattern in AAA waveform cycle with significant proximal disease.

a)

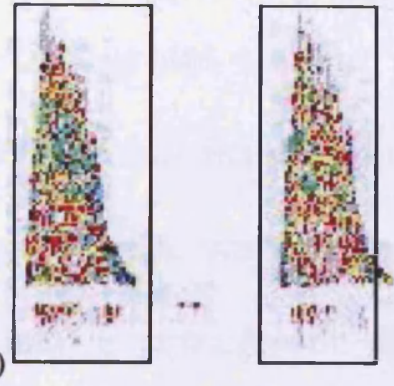

b)

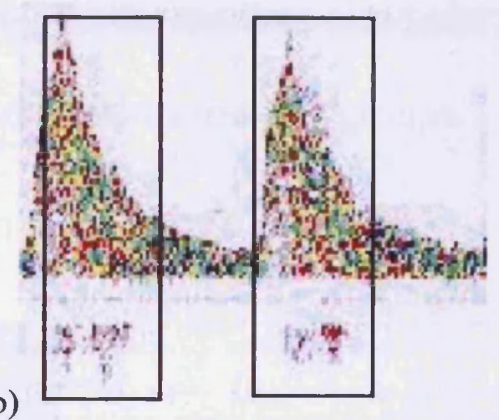

Figure 5.16: Simultaneous forward and reverse flow in a) AAA and b) no AAA waveform cycles with significant proximal disease.

It was not possible to determine whether the features observed in the AAA waveforms with proximal disease resulted from flow disturbances set up within the AAA, or whether they were caused by the presence of atherosclerotic disease. In the case of an occlusion of the iliac artery, the features in the CFA waveform are unlikely to be due to an AAA because the blood flow below the level of reconstitution is reliant upon collateral circulation. In the case of an iliac stenosis, the waveform changes are also more likely to be related to the flow disturbances associated with a stenosis rather than an AAA. 


\subsubsection{AAA Detection in Patients with Significant Proximal Atherosclerotic Disease}

\section{Using CFA Visual Feature Analysis}

In order to determine the feasibility of detecting AAA by visual feature analysis of CFA waveforms for patients in the proximal atherosclerotic disease groups, the right and left CFA waveforms each patient (including the 3 waveforms in the AAA group and 4 waveforms in the no AAA group without proximal disease) were scored according to the presence of the 4 visual features discussed in the previous section. Figure 5.17 shows the number of CFA waveforms from the AAA and no AAA patients in the significant proximal atherosclerotic disease groups with each feature present. It can be seen that none of the features provide a good level of discrimination between the AAA and no AAA patients in these groups.

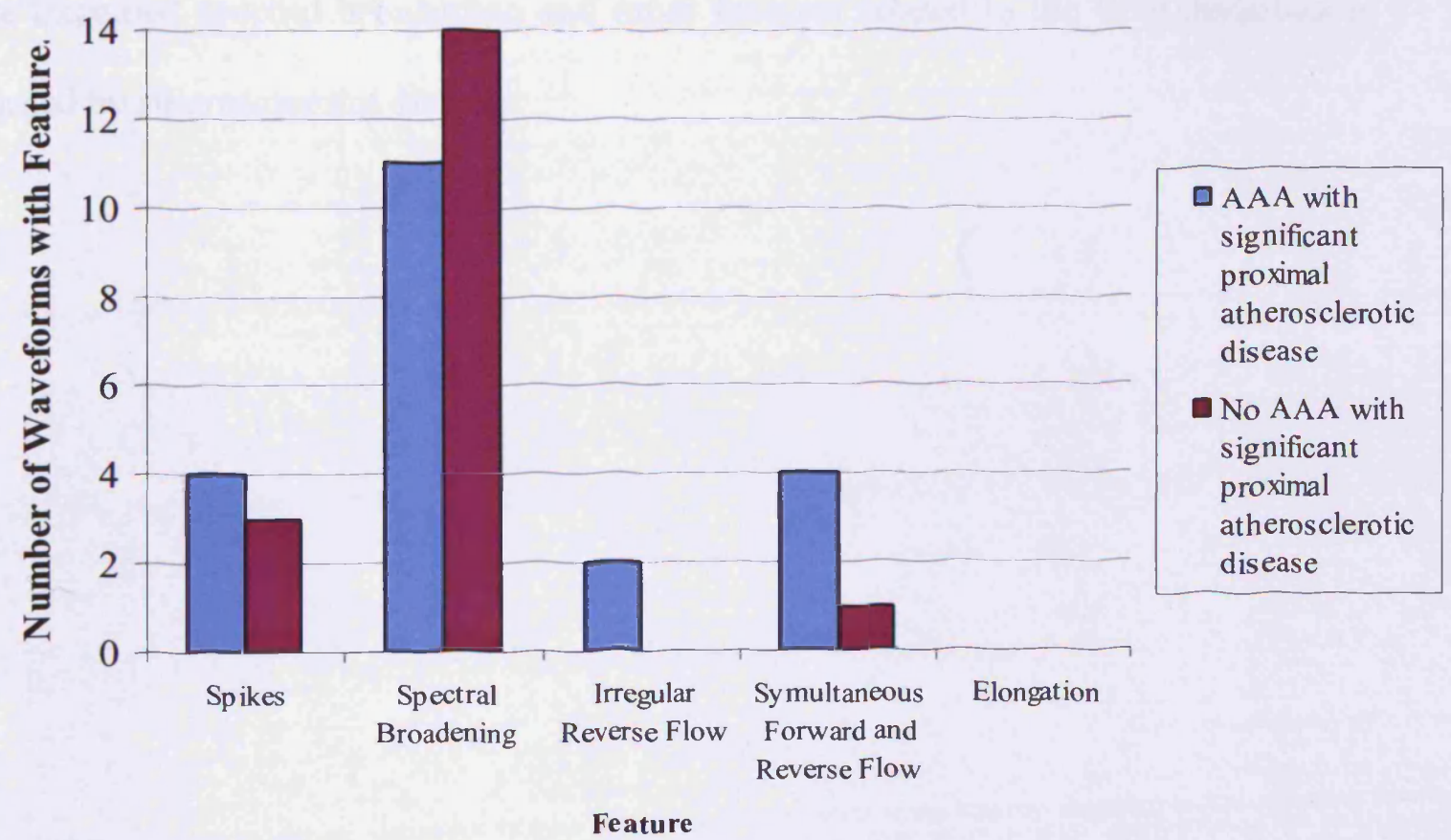

Figure 5.17: Number of CFA waveforms out of 11 AAA and 14 no AAA CFA waveforms in the significant proximal atherosclerotic disease groups with each feature present visually 
Again, the total scores for the CFA waveforms from the left and right legs were summed to give a total factor score per patient to try and improve the discrimination. The total scores for the $6 \mathrm{AAA}$ and 7 no AAA patients are plotted in Figures 5.18a and $5.18 \mathrm{~b}$. The mean factor score per patient in the AAA group was 3.5 with a maximum score of 4 factors and a minimum score of 2 factors. The mean total factor score per patient in the no AAA group was 2.6 with a maximum score of 4 factors and a minimum score of 2 factors.

The mean score for this proximal disease AAA group is lower than the mean scores for the no significant disease and significant distal disease AAA groups. This may be because the features in the waveforms related to AAA flow disturbances are not transmitted through an iliac stenosis or collateral vessels. The mean score in the no AAA proximal disease group is slightly increased and this is most likely to be due to the increased spectral broadening and other features related to the flow disturbances caused by atherosclerotic disease. 


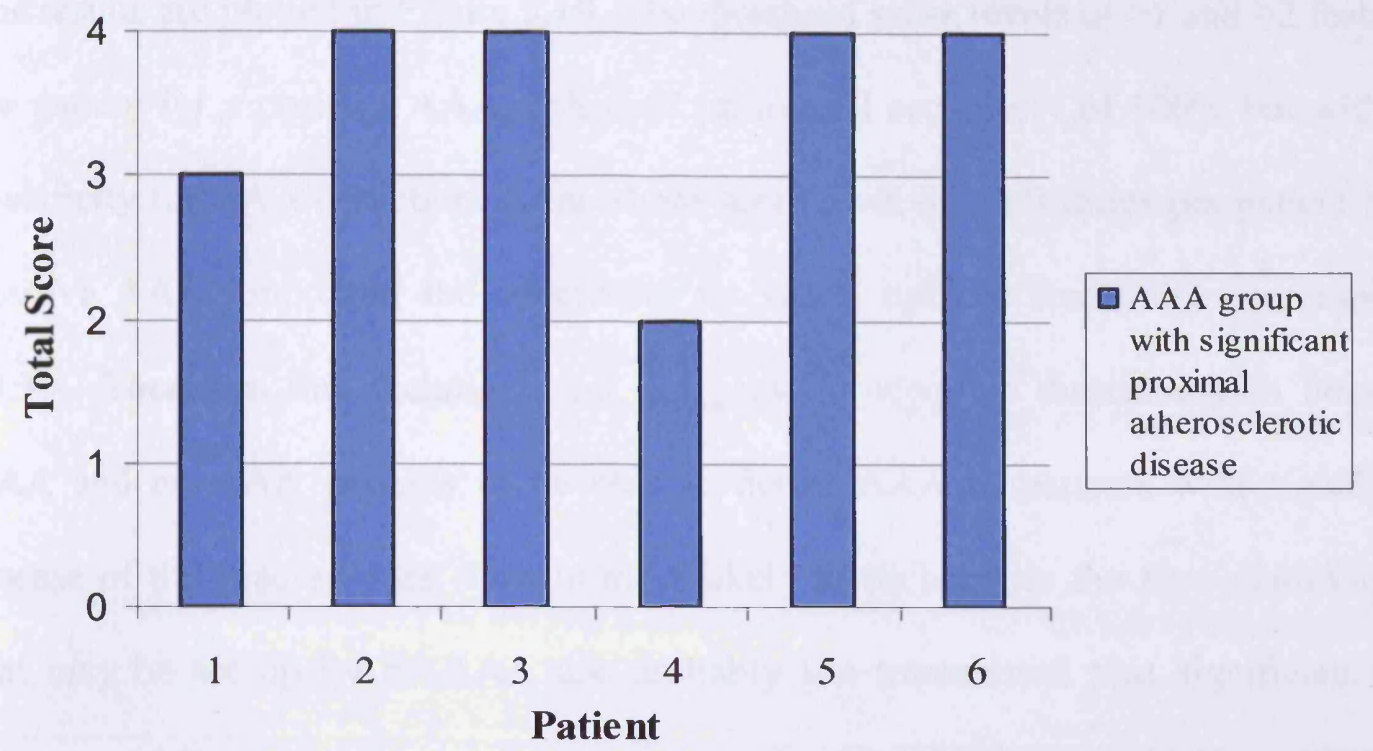

Figure 5.18a: Total Patient Scores for the 6 AAA patients with significant proximal atherosclerotic disease.

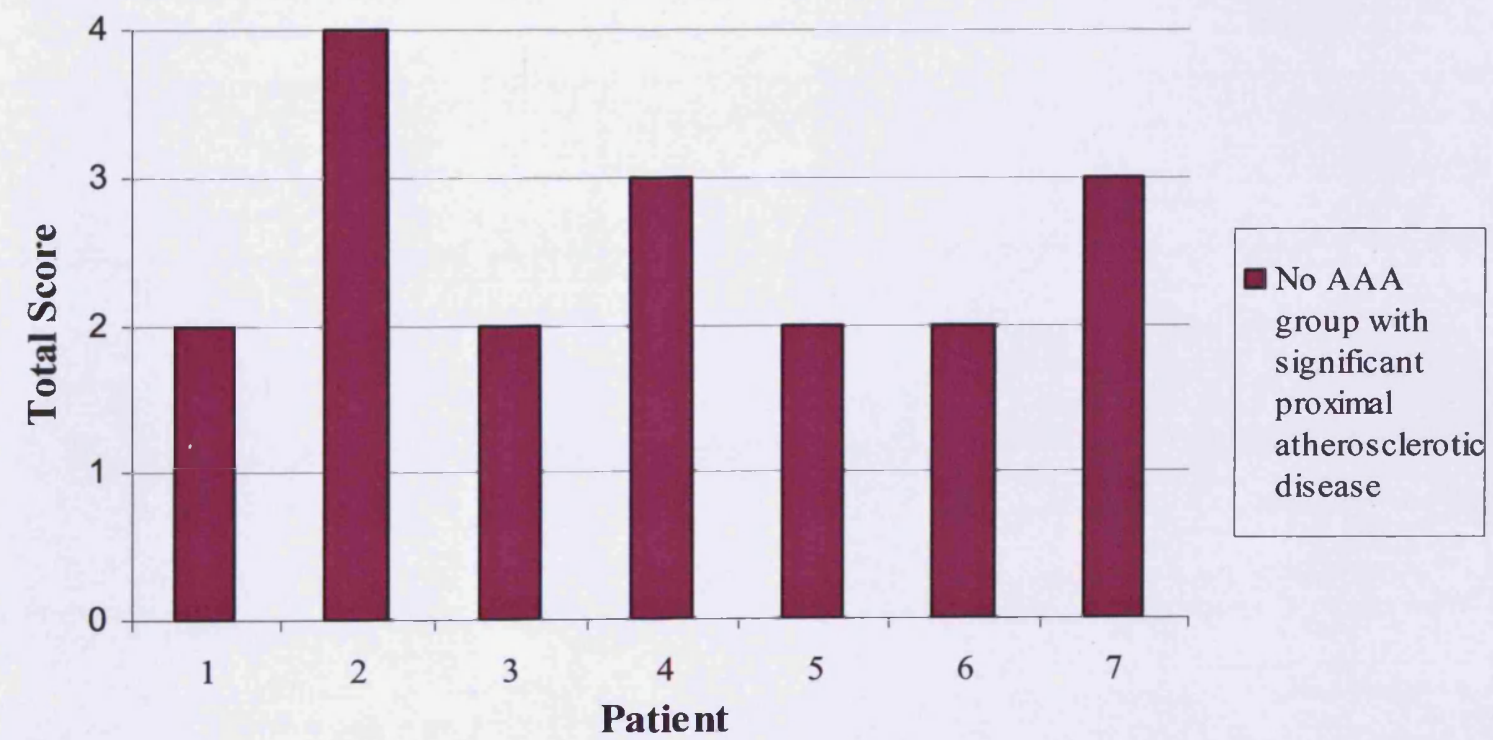

Figure 5.18b: Total Patient Scores for the 7 no AAA patients with significant proximal atherosclerotic disease. 
Again, the threshold levels of $\geq 1, \geq 2$ and $\geq 3$ were applied to the total patient score. The results are plotted in Figure 5.19. The threshold score levels of $\geq 1$ and $\geq 2$ features per patient for a positive AAA, achieved an overall sensitivity of $100 \%$ but with no specificity for AAA detection. A threshold score level of $\geq 3$ features per patient for a positive AAA, improved the specificity to $57.1 \%$ but the sensitivity decreased to 83.3\%. Therefore this technique did not provide adequate discrimination between AAA and no AAA patients to be able to detect AAA in patients with significant disease of the iliac arteries. This is most likely to be because the flow disturbances that may be set up by an AAA are probably not transmitted past significant iliac disease, and flow disturbances set up as a result of the atherosclerotic disease in both the AAA and no AAA groups make it almost impossible to discriminate between AAA and no AAA patients with significant proximal disease by visual analysis of the CFA waveform. 


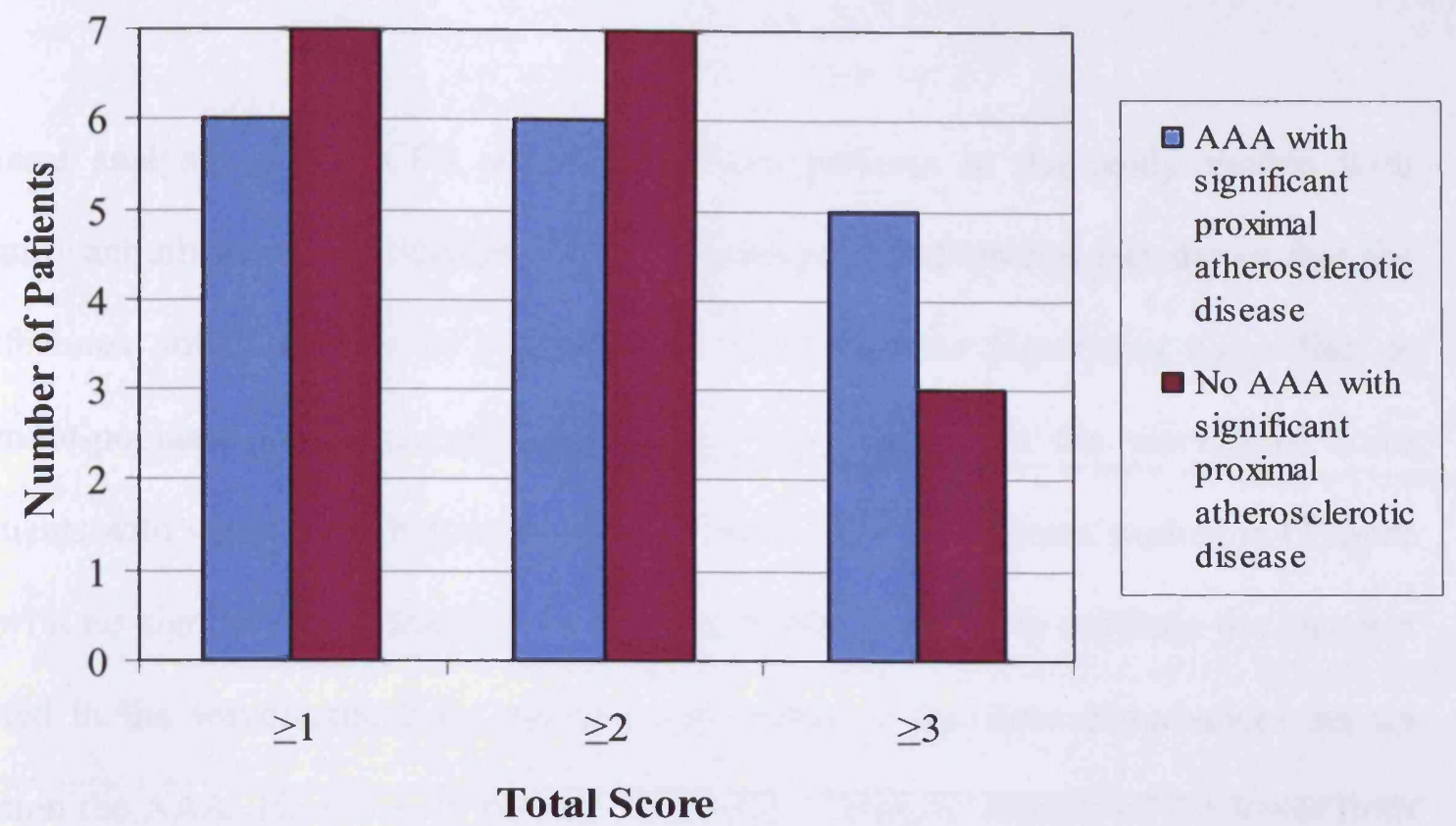

Figure 5.19: Number of AAA $(n=6)$ and no AAA $(n=7)$ patients with significant proximal atherosclerotic disease with $\geq 1, \geq 2$ and $\geq 3$ features present. 


\subsection{Discussion}

Visual analysis of the CFA waveforms from patients in the study groups with significant atherosclerotic disease of the femoro-popliteal arteries has shown that the 5 features noted visually in patients with AAA, but no significant aorto-iliac or femoro-popliteal atherosclerotic disease, are also present in the waveforms from patients with significant femoro-popliteal disease. For the patients studied in Chapter 3 with no significant atherosclerotic disease, it was possible to attribute the changes noted in the waveforms from patients with AAA to the flow disturbances set up within the AAA. However, in patients with atherosclerotic disease of the lower limb arteries, flow disturbances are also set up by the presence of atheromatous plaques and occlusions. All patients in the significant distal disease groups had at least one significant stenosis or occlusion of the femoro-popliteal arteries. In addition, the majority of patients also had extensive, diffuse calcification on the arterial walls throughout the iliac and femoro-popliteal arteries that may also disturb the blood flow. Therefore, in patients with AAA and significant atherosclerotic disease of the femoro-popliteal arteries, it was not possible to attribute the waveform changes solely to the AAA. Many of the waveforms from patients without AAA with significant distal disease also displayed similar changes. Therefore visual analysis of the CFA waveforms based on the 5 features of spectral broadening on the systolic down stroke, the appearance of spikes on the systolic down stroke, an irregular reverse flow pattern, simultaneous forward and reverse flow during systole and elongation of the waveform during the cardiac cycle, provided sensitivity to AAA of $100 \%$ but with a low specificity of $27.7 \%$ due to additional flow disturbances set up by atherosclerotic lesions. 
Visual analysis of CFA waveforms from AAA and no AAA patients with significant iliac artery disease showed that the 5 features displayed in the no significant arterial disease and significant distal disease groups were almost completely removed. The features that were present in the waveforms were more likely to be set up by the atherosclerotic arterial disease rather than AAA. It was therefore not possible to adequately discriminate between the AAA and no AAA patients with significant disease of the iliac arteries by visual feature analysis of the CFA waveform.

The additional changes to the CFA waveforms set up in the presence of significant iliac or femoro-popliteal atherosclerotic disease made it much more difficult to discriminate between patients with and without AAA by visual analysis of the CFA waveforms. Because visual analysis is subjective, it was aimed in Chapter 6 of this thesis to improve on the specificity by performing more objective analysis on these data sets using the MATLAB based AAA detection program designed in Chapter 4. 
Chapter 6: Objective Analysis of CFA Waveforms from AAA and No AAA

Patients with Significant Atherosclerotic Disease using MATLAB Software

\subsection{Introduction and Aim}

In Chapter 4, the MATLAB based program to detect AAA by analysis of CFA waveform features produced excellent results for patients with no significant atherosclerotic iliac or femoro-popliteal disease. The aim of the work in this chapter was to apply this method to objectively analyse the CFA waveforms from patients with significant iliac or femoro-popliteal atherosclerotic arterial disease, based on the features noted in visual inspection of the waveforms in Chapter 5, to try and improve on the results achieved for AAA detection by the visual analysis method.

In the first stage of the work, described in section 6.2 of this chapter, the CFA waveform files from the patients with and without AAA in the study groups with significant femoro-popliteal atherosclerotic disease were subjected to the AAA detection program that was designed and tested for patients without significant atherosclerotic disease in Chapter 4. Based on the outcome, in section 6.3, the selection of indices and threshold levels implemented by the detection program were adjusted to try to improve the performance of the program for these patients. In section 6.4, the performance of the modified detection program for the AAA and no AAA patient study groups with significant femoro-popliteal arterial disease is discussed. In section 6.5 , the performance of the modified detection program was tested by its response to the blind test sets of waveform data from the AAA and no AAA patients with significant femoro-popliteal atherosclerotic disease that were 
separated into the test groups. In section 6.6, the CFA waveforms from patients with and without AAA with significant iliac disease were subjected to the program. A discussion of the results and performance of the AAA detection program are given in section 6.7 .

\subsection{Performance of the AAA Detection Program for Patients with Significant}

\section{Femoro-Popliteal Atherosclerotic Disease}

In order to test the performance of the AAA detection program for patients with significant femoro-popliteal atherosclerotic disease, the CSV CFA waveform files from the $15 \mathrm{AAA}$ and 18 no AAA patients in the study groups with significant femoro-popliteal disease were subjected to the program.

\subsubsection{Preliminary Tests}

Before the waveforms were subjected to the AAA detection program, the initial data processing implemented by the program (described in section 4.3) was performed on each waveform file individually to ensure the program functioned correctly for each waveform. The sample regions for the automatic thresholding for background noise reduction were plotted to check their position. The maximum, minimum and bandwidth envelopes were plotted and visually inspected. The location markers to indicate the systolic peak and pulse foot, and the start and end of the reverse flow phases were plotted and superimposed on the spectrogram to check their position. 
The initial data processing operated correctly for all of the waveform files in the AAA and no AAA significant femoro-popliteal disease study groups.

\subsubsection{Initial AAA Detection Program Performance Results}

The left and right CFA waveforms from the 15 AAA and 18 no AAA patients were entered automatically into the AAA detection program in sequence. The individual scores that were calculated by the program for these patients are plotted in Figures 6.1a and $6.1 \mathrm{~b}$ respectively. The mean score per patient in the AAA group was 5.1 with a maximum score of 10 and a minimum score of 3 factors. The mean score per patient in the no AAA group was 4.8 with a maximum score of 9 and minimum score of 0 . These results were compared to those obtained for the no significant atherosclerotic disease study groups in Chapter 4 (Section 4.6). The mean score of 5.1 in the AAA distal disease group is slightly higher than the mean score of 4.8 in the AAA with no significant disease group. The mean score in the no AAA distal disease group of 4.8 is significantly higher than the mean score of 1.2 in the normal group.

Figure 6.2 shows the number of AAA and no AAA patients with $\geq 1, \geq 2$ and $\geq 3$ features present. 


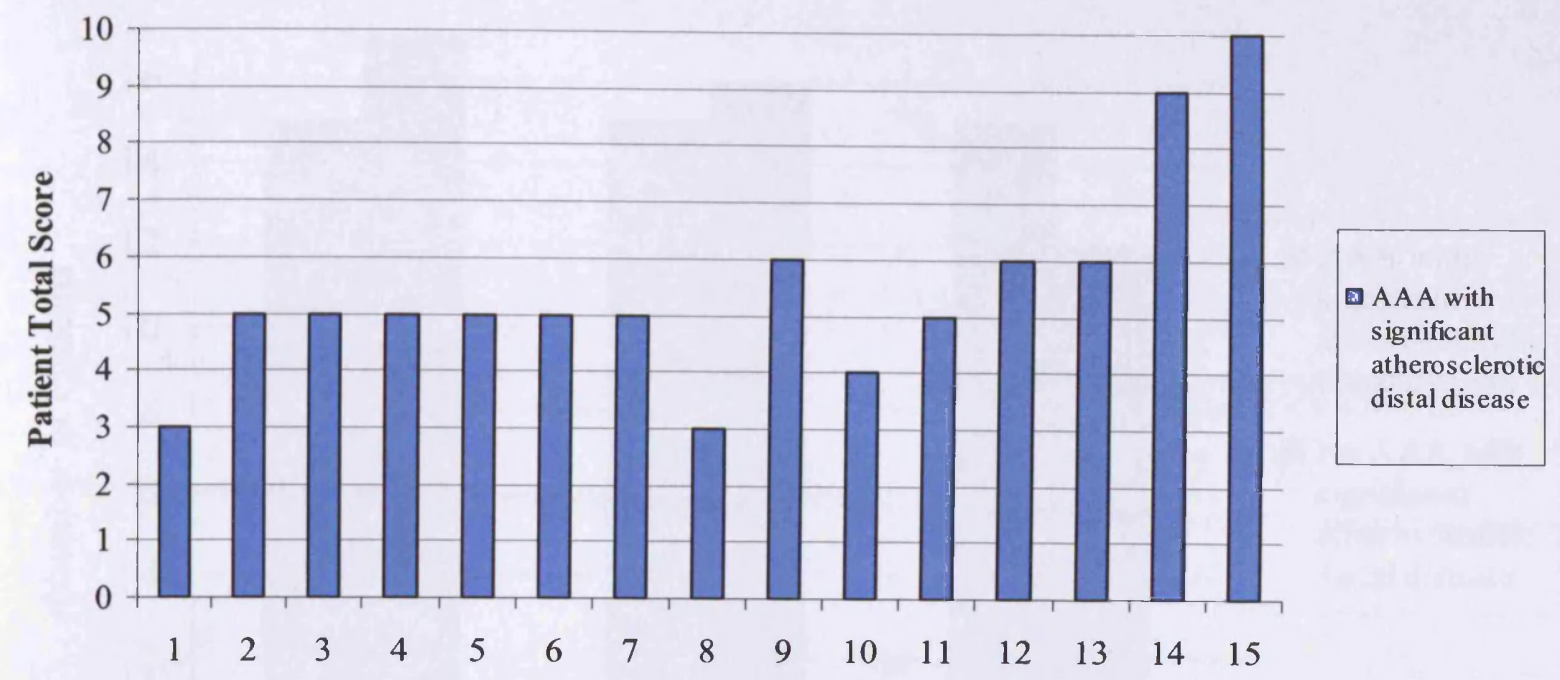

Patient

Figure 6.1a: Total Patient Scores for the 15 AAA patients with significant femoro-popliteal atherosclerotic disease.

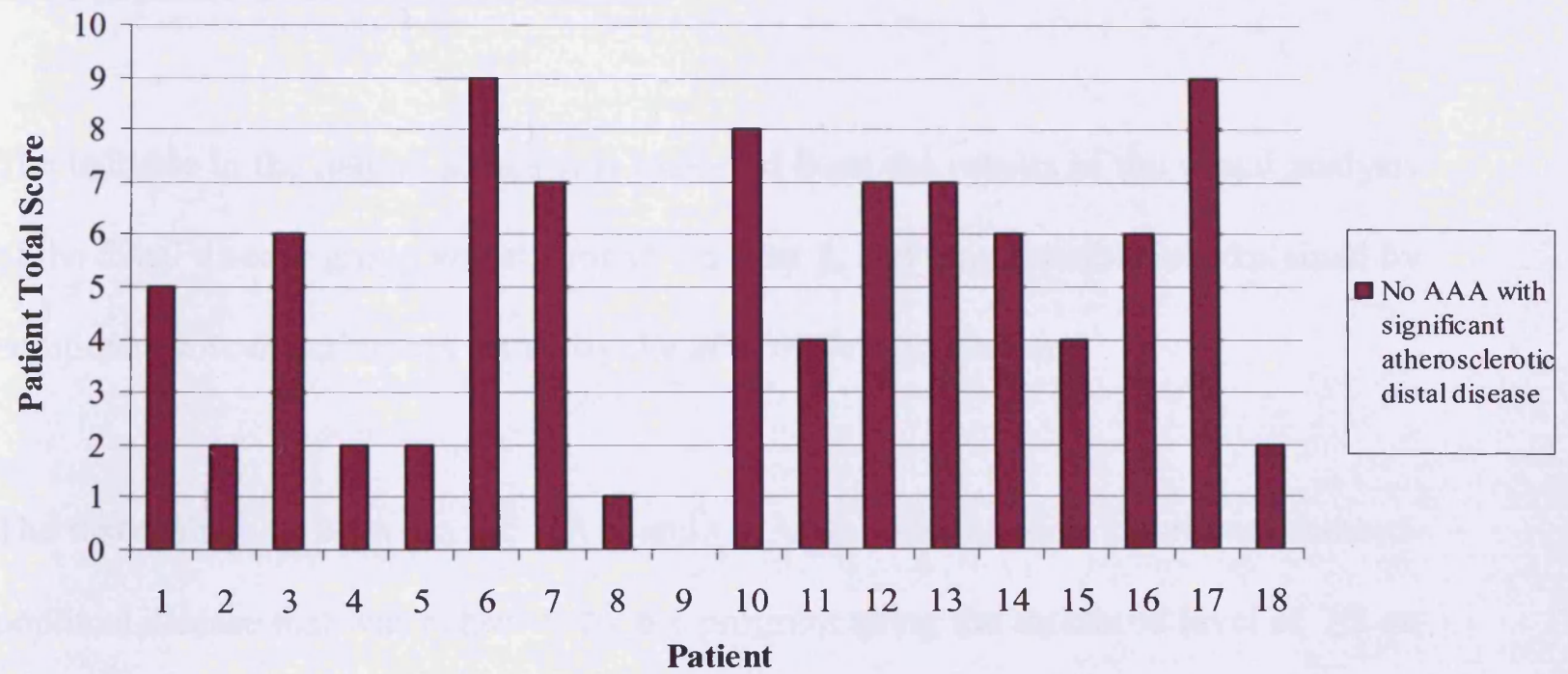

Figure 6.1b: Total Patient Scores for the 18 No AAA patients with significant femoro-popliteal atherosclerotic disease. 


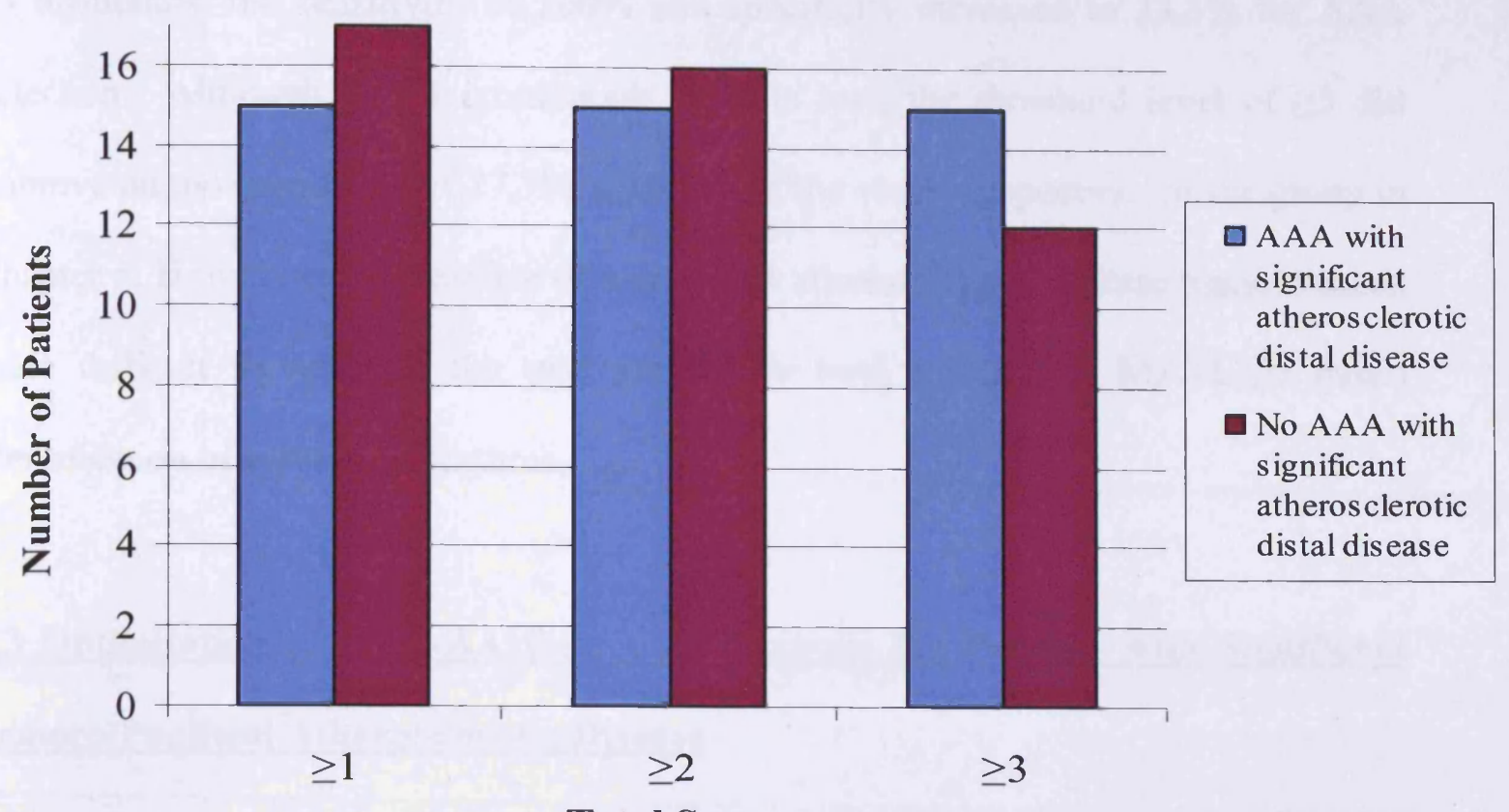

Total Score

Figure 6.2: Number of AAA $(n=15)$ and no AAA $(n=18)$ patients with significant femoro-popliteal atherosclerotic disease with $\geq 1, \geq 2$ and $\geq 3$ features present.

\subsubsection{Initial Program Results: Discussion}

The increase in the patient scores was expected from the results of the visual analysis of the distal disease group waveforms in Chapter 5, and may possibly be explained by additional flow disturbances set up by the atherosclerotic disease.

The discrimination between the AAA and no AAA patients with significant femoropopliteal disease that was achieved by the program using the threshold level of $\geq 2$ on the patient scores between the AAA and no AAA distal disease groups was much poorer than was achieved for the no significant disease group.

The threshold score levels of $\geq 1$ and $\geq 2$ achieved a sensitivity of $100 \%$ with but with very low specificities of $5.6 \%$ and $11.1 \%$ respectively. The threshold score level of 
$\geq 3$ maintained the sensitivity of $100 \%$ and specificity increased to $33.3 \%$ for AAA detection. Although the discrimination level is low, the threshold level of $\geq 3$ did improve on the specificity of $27.7 \%$ achieved in the visual inspection for the group in Chapter 5. However, the presence of significant atherosclerotic disease made it much more difficult to separate the two groups by both visual and MATLAB based identification of waveform features.

\subsection{Optimisation of the AAA Detection Program for Patients with Significant}

\section{Femoro-Popliteal Atherosclerotic Disease}

In order to try to improve the discrimination between the AAA and no AAA patients in the significant femoro-popliteal atherosclerotic disease groups, the levels for each of the 6 indices calculated by the AAA detection program (1) Maximum Systolic Down-Stroke Bandwidth Index, 2) Systolic Spike Index, 3) Reverse Power Density Index, 4) Reverse to Systolic Height Ratio Index, 5) Integral Reverse Power Under Systolic Upstroke Index, 6) Integral Reverse Power Under Systolic Down-Stroke Index) for the left and right CFA waveforms from the $15 \mathrm{AAA}$ and 18 no AAA patients in the significant Femoro-Popliteal disease groups were plotted and visually inspected. The aims were: 1) to determine whether the indices were suitable for the waveforms in these groups; and 2) to determine whether the threshold levels for the indices set by the original program provided adequate discrimination between the groups, or whether the level could be improved by adjusting the threshold levels.

A new MATLAB program referred to as the 'disease group AAA detection program' was set up to test different combinations of the indices, threshold levels and weighting 
factors with the aim of improving on the performance of the original program for the significant distal disease patients. This new program operated in the same way as the original AAA detection program as described in Figure 4.22 (Chapter 4, Section 4.6).

\subsubsection{Maximum Systolic Down-Stroke Bandwidth Index in the Distal Disease Groups}

The Maximum Systolic Down-Stroke Bandwidth Index levels for the left and right CFA waveforms for the $15 \mathrm{AAA}$ and 18 no AAA patients in the significant distal disease study groups are plotted in Figure 6.3a. The threshold level set by the original AAA detection program is indicated by the dotted black line.

The range of levels for this index within these two study groups is similar to the range in the no significant disease study groups plotted in Figure 4.12 (Chapter 4). The index level for $43.3 \%$ of AAA (13 out of 30 ) waveforms and $27 \%$ of no AAA (10 out of 36) waveforms in the distal disease groups lay above the threshold of 0.76 . This discrimination level is not as good as it was for the no significant disease groups for which $55 \%$ of AAA (33 out of 60 ) waveforms and $26.7 \%$ of normal (16 out of 60 ) waveforms lay above the threshold.

On visual inspection of Figure $6.3 \mathrm{a}$ the threshold is reasonably well placed. However, to increase the discrimination between the AAA and no AAA distal disease groups a number of different, increased, threshold levels were tested in the disease group AAA detection program index combinations. The best level of discrimination was provided with a threshold level of 0.80 as indicated by the dotted green line. 


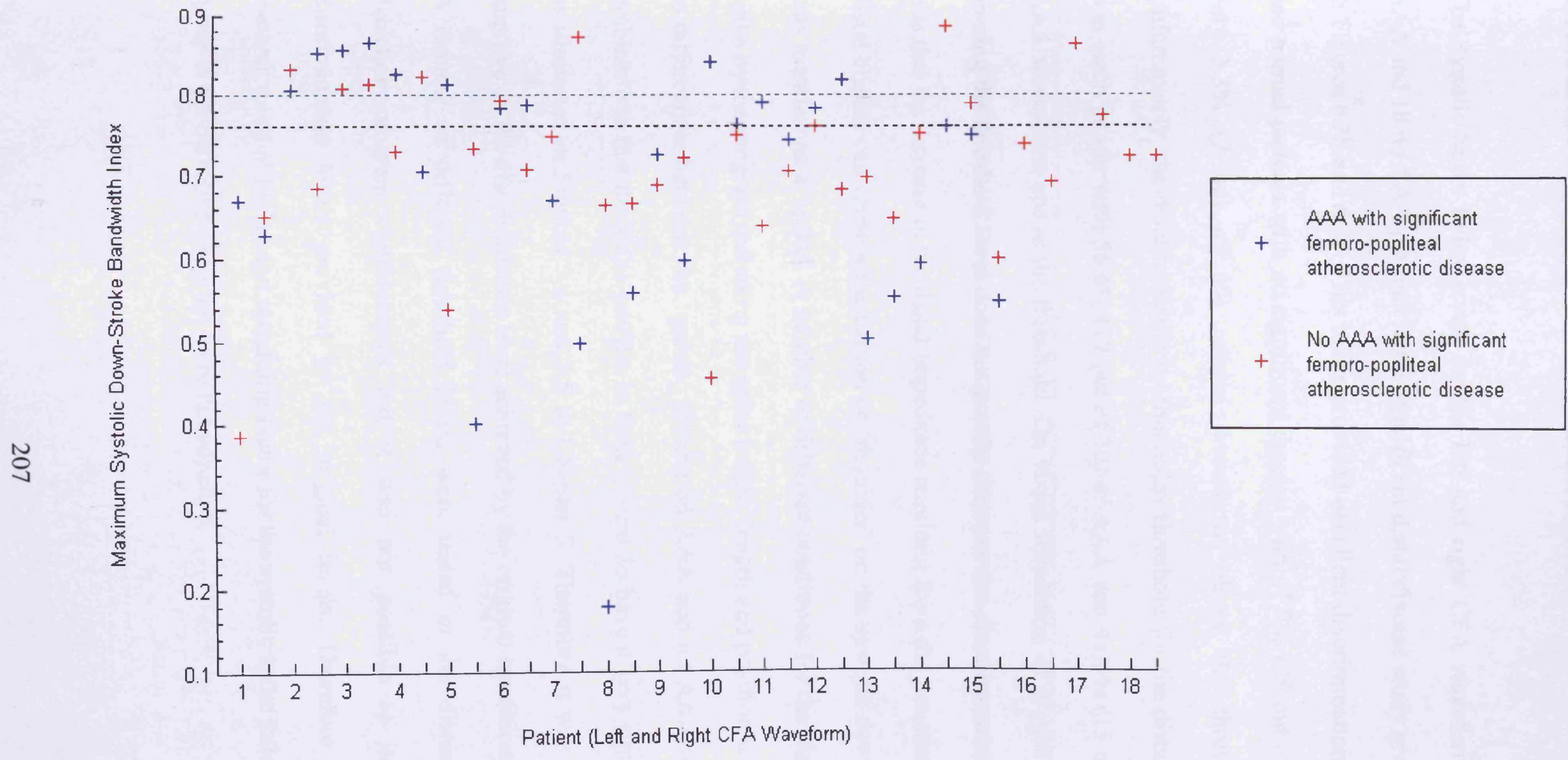

Figure 6.3a: Maximum Systolic Down-Stroke Bandwidth Index plotted for the left and right CFA waveforms for the 15 AAA and 18 no AAA patients in the significant femoro-popliteal atherosclerotic disease study groups. 


\subsubsection{Systolic Spike Index in the Distal Disease Groups}

The Systolic Spike Index levels for the left and right CFA waveforms from the 15 AAA and 18 no AAA patients in the significant distal disease study groups are plotted in Figures $6.3 \mathrm{~b}$ and $6.3 \mathrm{c}$. This index provided excellent discrimination between AAA and normal patients with no significant disease with $41.7 \%$ (25 out of 60 ) AAA and only $3.3 \%$ ( 2 out of 60 ) normal waveforms above the threshold of $\geq 0.5$. Unfortunately, the discrimination by this index threshold for the distal disease group was much poorer with $56.6 \%$ (17 out of 30 ) of AAA and $41.7 \%$ (15 out of 36$)$ of no AAA waveforms above the threshold. On visual inspection of graphs $6.3 \mathrm{~b}$ and $6.3 \mathrm{c}$, altering the threshold level does not greatly improve the discrimination. The problem was that the increase in the distal impedance resulting from the presence of significant distal disease can cause a fluctuation or 'shoulder' on the systolic down-stroke which may manifest as a 'spike'. A number of different conditions for the classification of a spike by the program including the spike height, length and position were tested to try to differentiate between the 'spikes' present on AAA and no AAA waveforms. The problem was that the spike profiles in both groups do have a very similar appearance as illustrated in Figures 5.4 and 5.5 in Chapter 5. Therefore it was not possible to improve on the discrimination level achieved by the original conditions and threshold. A number of different threshold levels were tested in the disease group AAA detection program combinations but it was not possible to improve on the discrimination levels provided by the original levels. Therefore the calculation method, threshold level and weighting factor for the systolic spike index in the disease group AAA detection program were not adjusted. 


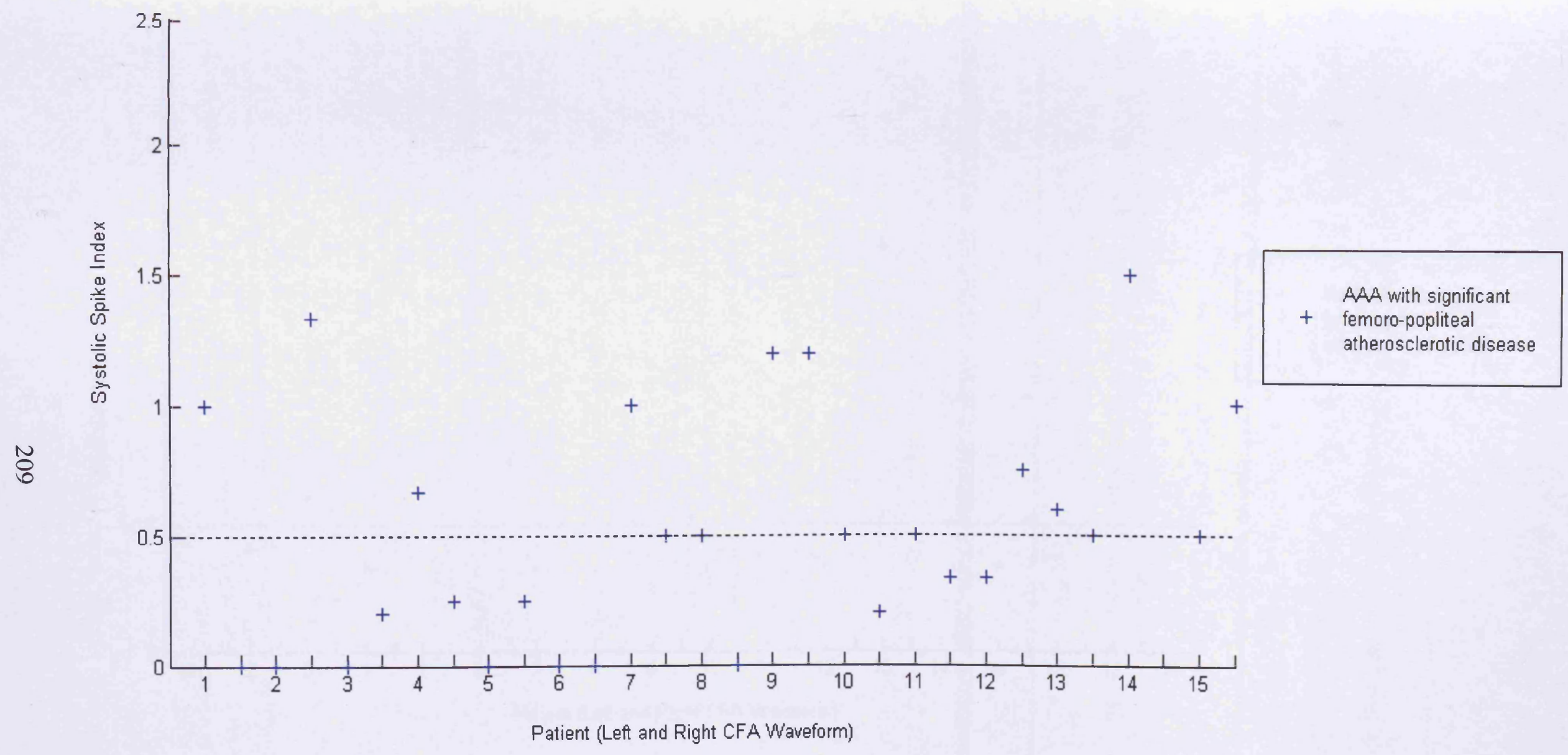

Figure 6.3b: Systolic Spike Index plotted for the left and right CFA waveforms for the 15 AAA patients in the significant femoro-popliteal atherosclerotic disease study group. 


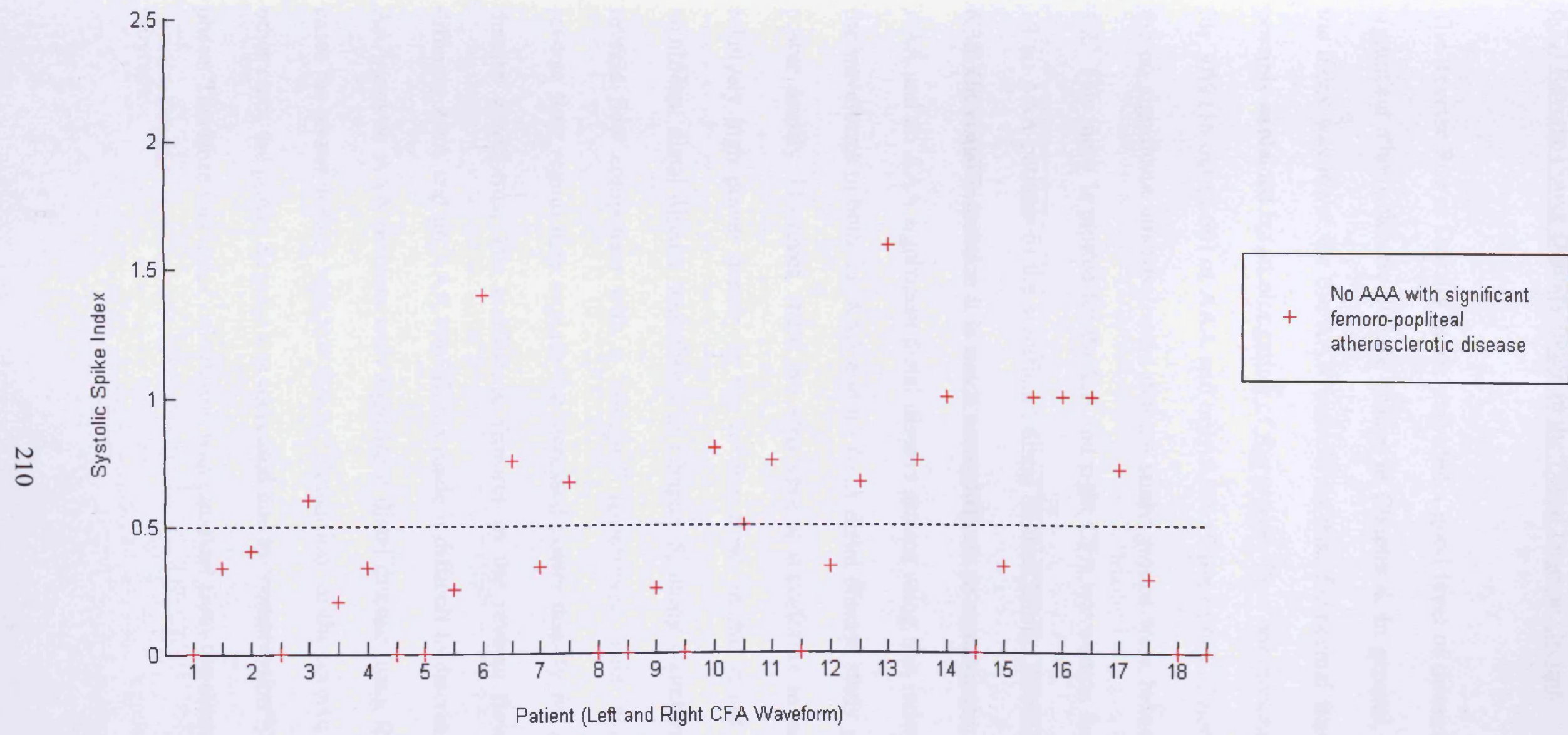

Figure 6.3c: Systolic Spike Index plotted for the left and right CFA waveforms for the 18 no AAA patients in the significant femoro-popliteal atherosclerotic disease study group. 


\subsubsection{Reverse Power Density Index in the Distal Disease Groups}

The Reverse Power Density Index provided a good level of discrimination for the no significant atherosclerotic disease groups in Chapter 4. In general, for these patients, the index was lower for the AAA waveforms than for normal waveforms. This was possibly explained by an elongation of the reverse flow component. The index levels for $30 \%$ (18 out of 60$)$ of AAA and only $8.3 \%$ (5 out of 60$)$ of normal waveforms in the no significant atherosclerotic disease study groups were below the threshold of 4.2. This index is plotted for the left and right CFA waveforms for the 15 AAA and 18 no AAA patients in the significant distal atherosclerotic disease groups in Figure 6.3d. On visual inspection it is much more difficult to separate the waveforms in the AAA and no AAA significant distal disease groups using this index. The majority of the waveforms in both the AAA and no AAA distal disease study groups have a low power density. However, there are also several waveforms in each group with a relatively high power density in the reverse flow. In the visual inspection of the significant distal disease waveforms in Chapter 5, many waveforms had a reduced reverse flow component with a 'stumped' appearance. This 'compression' of the reverse flow region may explain the increased power density for some of the distal disease waveforms. The conflicting features in the reverse flow components for different AAA and no AAA waveforms made it difficult to discriminate between the AAA and no AAA patients with significant distal disease using this index. In some cases the power density was low due to 'elongation' of the reverse flow phase and in other cases the power density was increased due to 'compression' of the reverse flow phase. Therefore this index calculation was removed from the disease AAA detection program. 


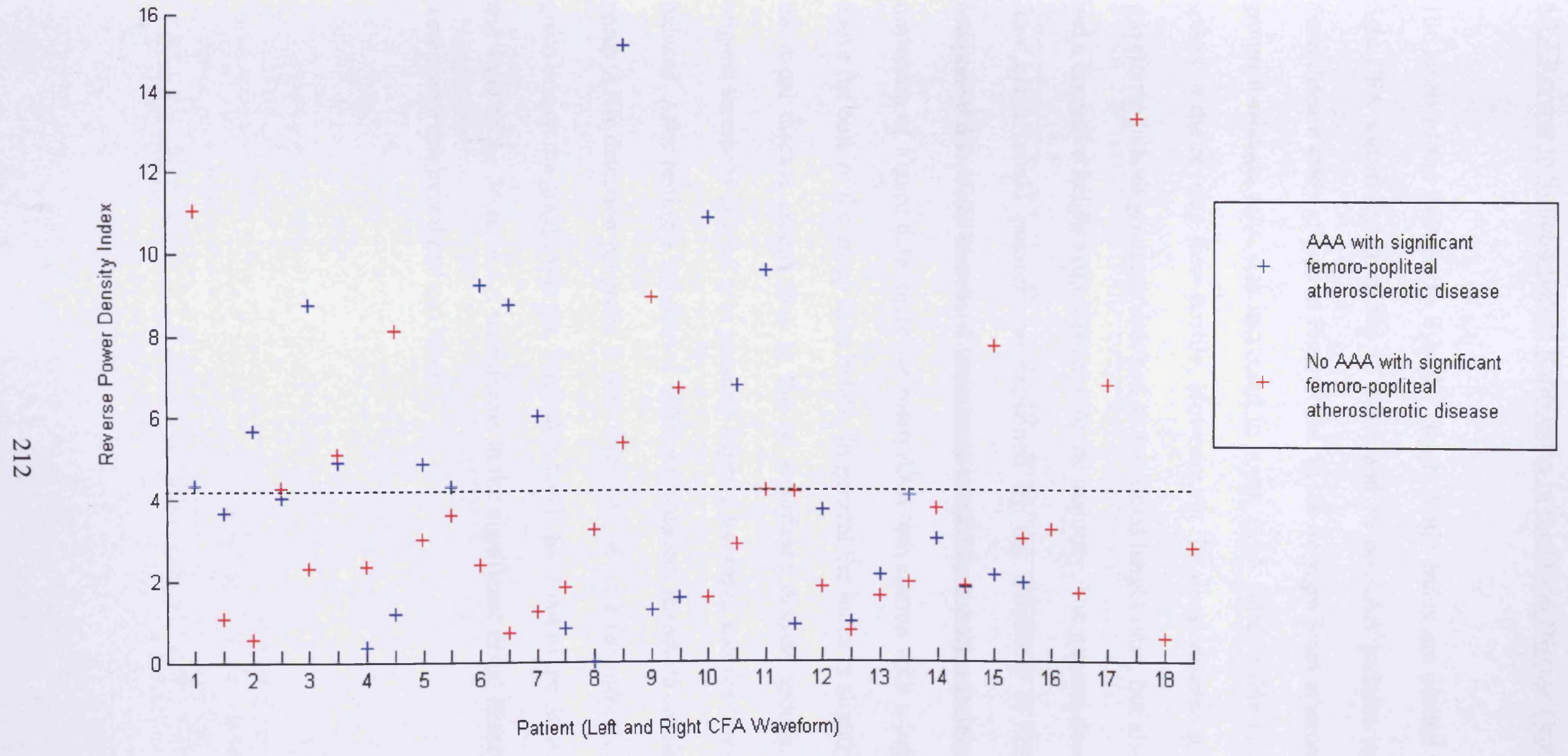

Figure 6.3d Reverse Power Density Index plotted for the left and right CFA waveforms for the 15 AAA and 18 no AAA patients in the significant femoropopliteal atherosclerotic disease study groups. 


\subsubsection{Reverse to Systolic Height Ratio Index in the Distal Disease Groups}

The levels of the Reverse to Systolic Height Ratio Index are plotted for the left and right CFA waveforms from the $15 \mathrm{AAA}$ and 18 no AAA patients in the significant distal disease study groups in Figure 6.3e. In the no significant atherosclerotic disease groups the height ratio was increased in some AAA patients due to the presence of spikes in the reverse flow profile. However, in the distal disease group there were waveforms in both groups which had an increased height ratio, but also several which had a decreased height ratio compared to the majority. The general decrease in reverse flow height could possibly be explained by the reduction of the reverse flow component due to the increased impedance caused by the atherosclerotic disease. On inspection of Figure $6.3 \mathrm{e}$, there are more AAA waveforms with a height ratio index above the bulk of the group than below. In general the index is slightly lower in both the distal disease groups than in the no significant disease groups. Therefore the original threshold of $>5.2$ was possibly slightly too high, and the threshold level was reduced. After testing a number of different, reduced, threshold levels in the disease group AAA detection program, a new threshold of $>4.2$ (as indicated by the dotted green line on the graph) was set. The index levels for 7 out of the 30 AAA waveforms and 4 out of the 36 no AAA waveforms in the significant distal disease study groups were above this new threshold level. 


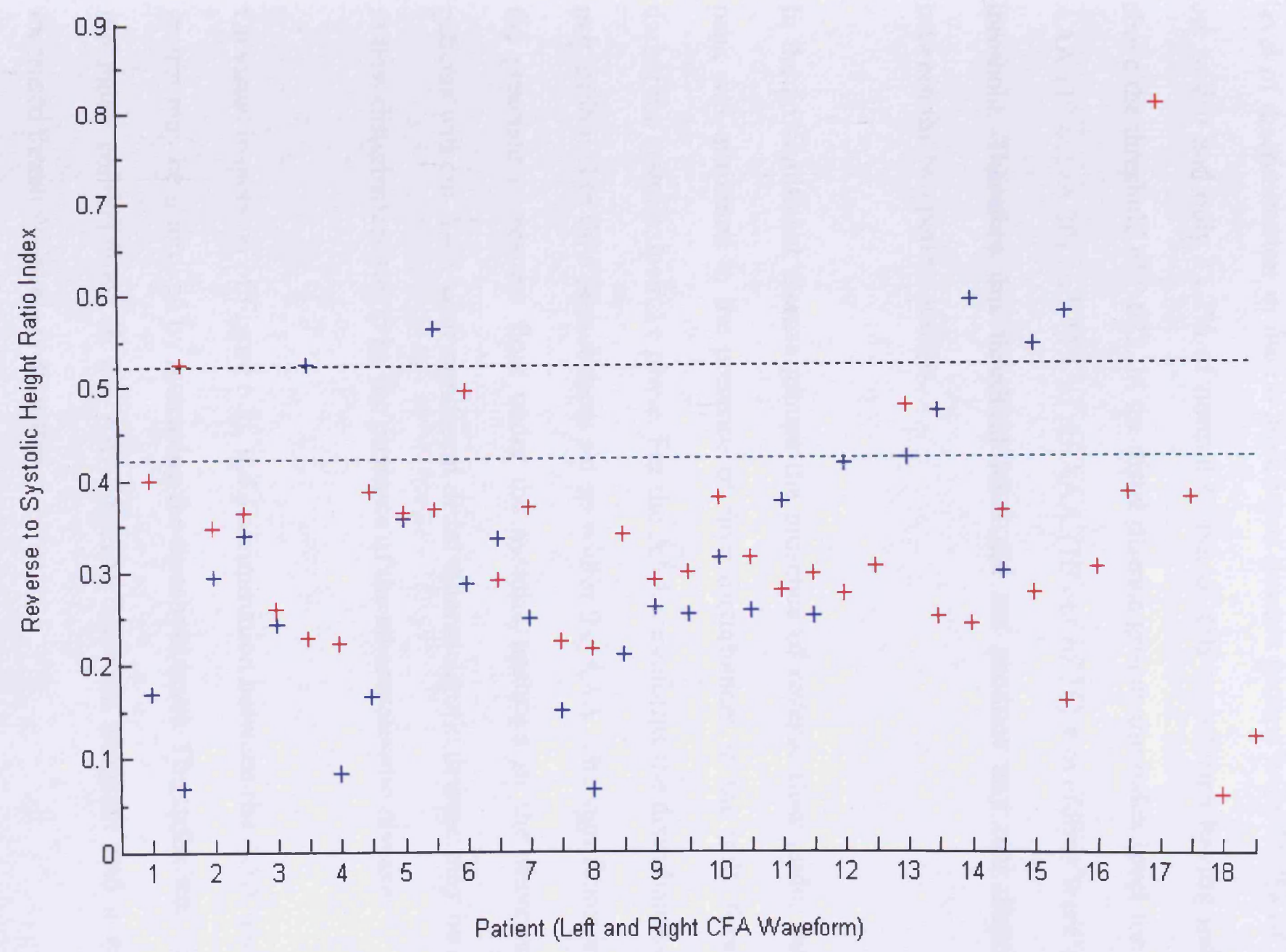

Figure 6.3e Reverse to Systolic Height Ratio Index plotted for the left and right CFA waveforms for the 15 AAA and 18 no AAA patients in the significant femoro-popliteal atherosclerotic disease study groups. 


\subsubsection{Integral of Reverse Power Under Systolic Upstroke Index in the Distal Disease}

\section{$\underline{\text { Groups }}$}

The levels of the Integral of the Reverse Power Under the Systolic Upstroke Index for the left and right CFA waveforms from the 15 AAA and 18 no AAA patients in the distal disease study groups are plotted in Figure 6.3f. This index provided a good level of discrimination in the no significant disease groups with $41.7 \%$ of AAA (25 out of 60 ) and only $11.7 \%$ of normal ( 7 out of 60 ) waveforms having index levels above the threshold of 0.025 . In the distal disease groups the index level for $33.3 \%$ of AAA (10 out of 30$)$ and $44 \%$ of no AAA (16 out of 36$)$ waveforms were above the threshold. Therefore this threshold level did not produce any real discrimination between the two patient groups.

In the no significant disease groups the presence of reverse flow under the systolic peak was attributed to the presence of flow disturbances in the bulk forward flow during the systolic upstroke phase. For the AAA waveforms the disturbances were inpart attributed to flow disturbances set up within the AAA. The significant increase in the presence of reverse flow under the systolic upstroke in the waveforms from patients without AAA with significant distal atherosclerotic disease may be attributed to flow disturbances set up by the presence of the atherosclerotic disease.

On visual inspection of Figure 6.3f, the discrimination between the AAA and no AAA groups may be improved by increasing the threshold level. The index was included in the index combinations in the AAA disease detection program and a number of increased threshold levels were tested. 
The threshold level was increased to 0.1 (as indicated by the dotted green line on the graph. The index levels for $23.3 \%$ (7 out of 30 ) of AAA waveforms and $16.7 \%$ (6 out of 36) of the no AAA waveforms in the significant distal disease study groups were above this new threshold level. 


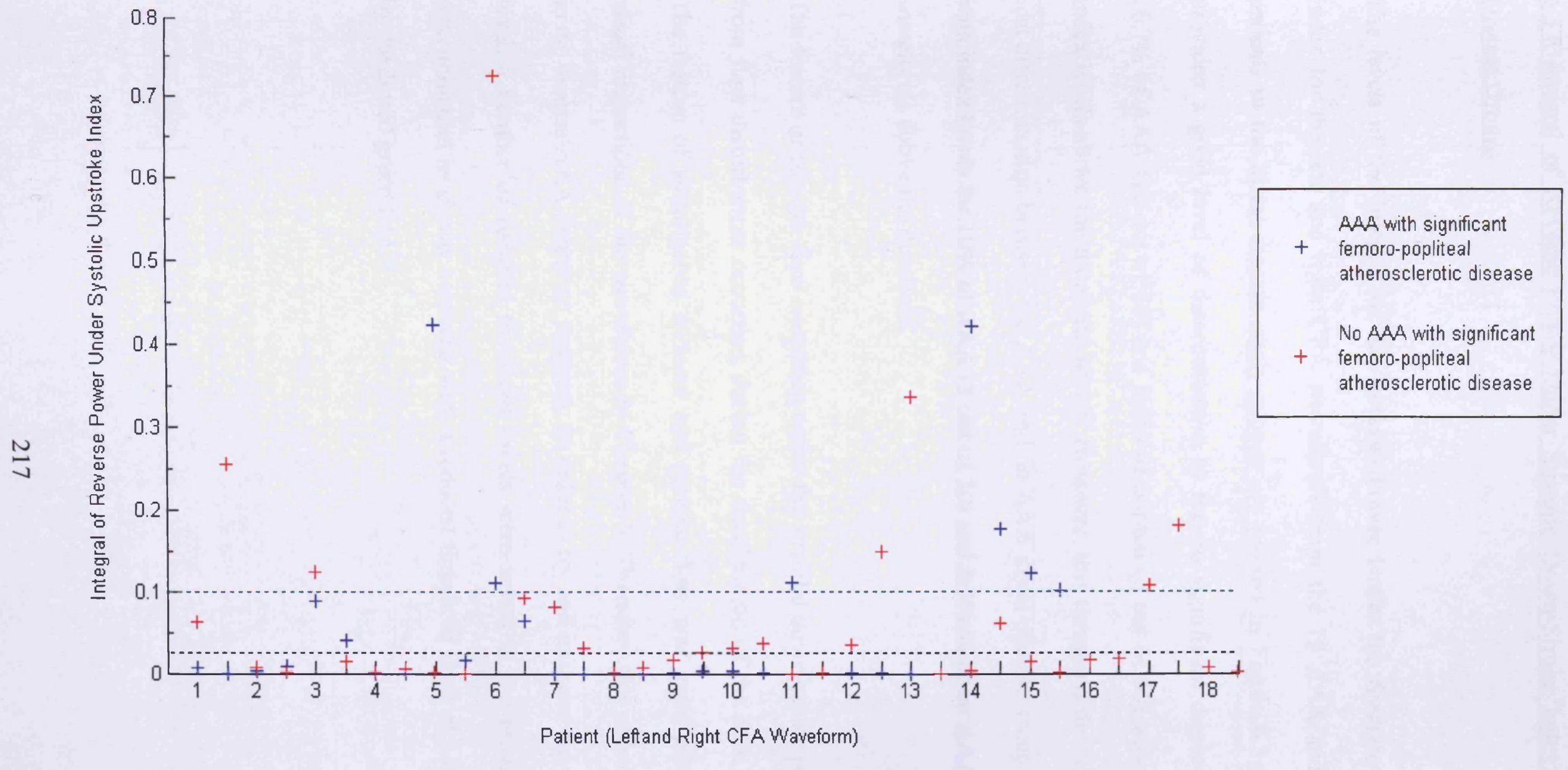

Figure 6.3f: Integral of the Reverse Power Under the Systolic Upstroke Index plotted for the left and right CFA waveforms for the 15 AAA and 18 normal patients in the significant femoro-popliteal atherosclerotic disease study groups. 


\subsubsection{Integral of Reverse Power Under Systolic Down-Stroke Index in the Distal}

\section{Disease Groups}

The levels of the Integral of the Reverse Power Under the Systolic Down-Stroke Index for the left and right CFA waveforms from the 15 AAA and 18 no AAA patients in the distal disease study groups are plotted in Figure $6.3 \mathrm{~g}$. This index provided a good level of discrimination in the no significant disease groups with $16.7 \%$ of AAA (10 out of 60 ) and $8.3 \%$ of normal (5 out of 60 ) waveforms having index levels above the threshold of 1.5. However, this threshold did not provide any real discrimination between the AAA and no AAA distal disease groups study groups with index levels for $10 \%$ of AAA ( 3 out of 30 ) and $8.3 \% \%$ of no AAA ( 3 out of 36 ) waveforms above the threshold.

The feature of reverse flow occurring under the systolic down-stroke possibly results from flow disturbances occurring during the deceleration of the bulk forward flow. The feature of simultanous forward and reverse flow was quite prominent in the visual inspections of the waveforms in Chapter 5. Therefore this index was included in the disease AAA detection program. In order to try and improve the discrimination level, a number of reduced threshold levels were tested in the program. The best discrimination level was achieved with a reduced threshold level of 1.25 as indicated by the dotted green line. 


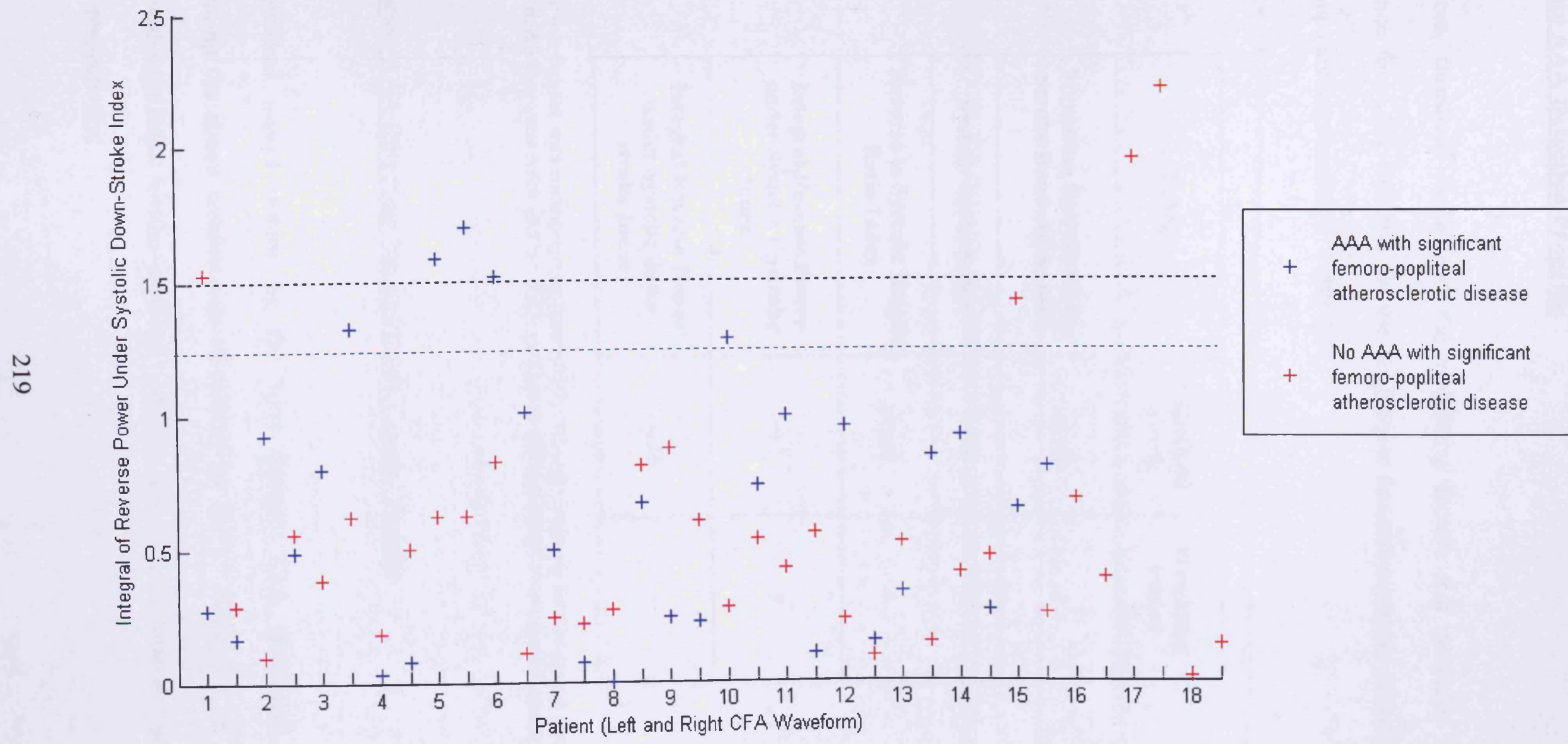

Figure 6.3g: Integral of the Reverse Power Under the Systolic Down-Stroke Index plotted for the left and right CFA waveforms for the 15 AAA and 18 normal patients in the significant femoro-popliteal atherosclerotic disease study groups. 


\subsection{Disease AAA Detection Program}

The indices, threshold levels and the weighting factors that provided the best performance for AAA detection in the significant femoro-popliteal (distal) disease study groups are summarised in Table 6.1.

\begin{tabular}{|c|c|c|}
\hline Index & $\begin{array}{c}\text { Threshold } \\
\text { Level }\end{array}$ & $\begin{array}{c}\text { Weighting } \\
\text { Factor }\end{array}$ \\
\hline $\begin{array}{c}\text { Maximum Systolic Down- } \\
\text { Stroke Bandwidth Index }\end{array}$ & $>0.80$ & 1 \\
\hline Systolic Spike Index & $\geq 0.5$ & 2 \\
\hline $\begin{array}{c}\text { Reverse to Systolic Height } \\
\text { Ratio Index }\end{array}$ & $>0.42$ & 1 \\
\hline $\begin{array}{c}\text { Integral Reverse Power } \\
\text { under Systolic Upstroke } \\
\text { Index }\end{array}$ & $>0.1$ & 1 \\
\hline $\begin{array}{c}\text { Integral Reverse Power } \\
\text { under Systolic down } \\
\text { stroke Index }\end{array}$ & $>1.25$ & \\
\hline
\end{tabular}

Table 6.1: Indices with corresponding threshold levels and weighting factors giving the best discrimination between AAA and No AAA patients in the significant distal disease study groups

\subsubsection{Disease AAA Detection Program Study Groups' Results}

The individual scores output by the new disease AAA detection program implementing the above combination of indices for the 15 AAA and 18 no AAA patients with significant femoro-popliteal occlusive disease are plotted in Figures 6.4a and $6.4 \mathrm{~b}$ respectively. 


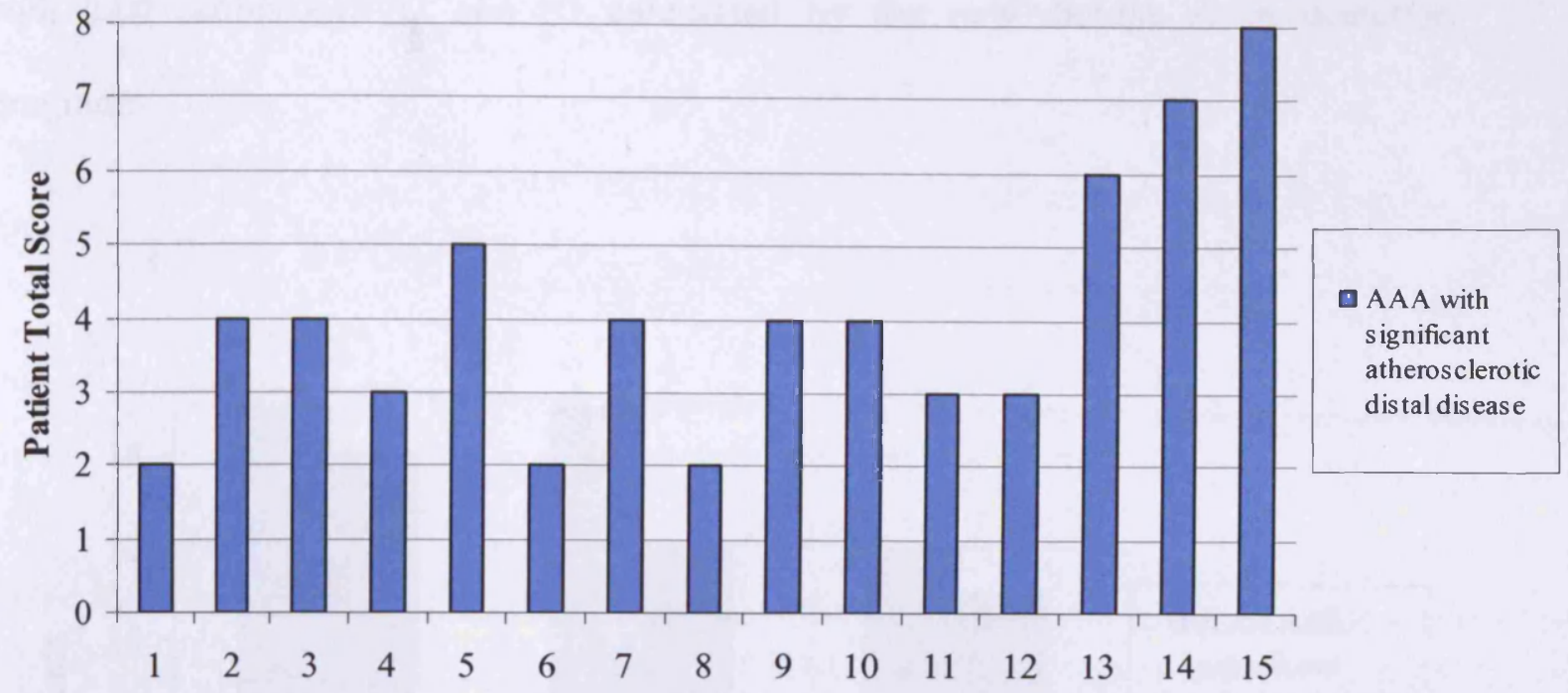

Patient

Figure 6.4a: Total Patient Scores for the 15 AAA patients with significant femoro-popliteal atherosclerotic disease.

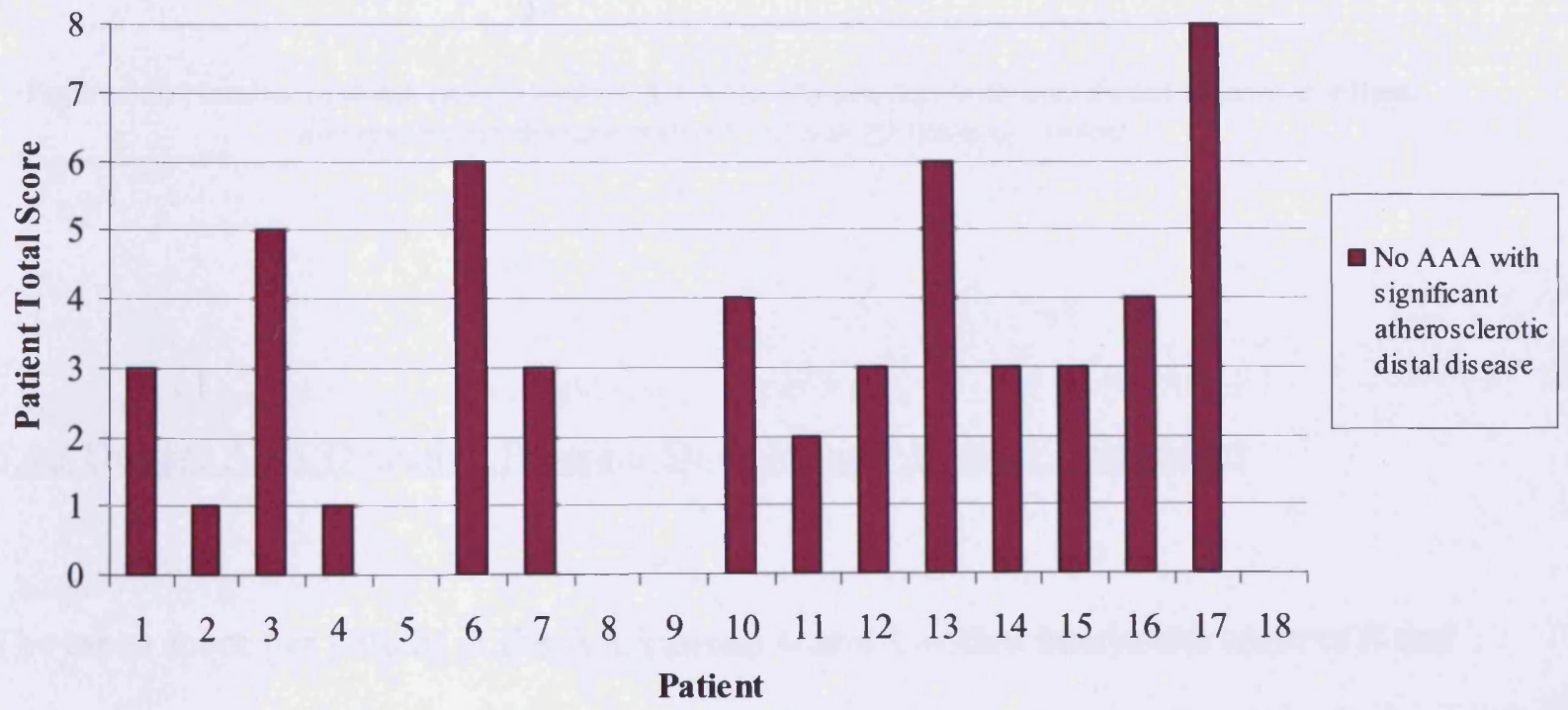

Figure 6.4b: Total Patient Scores for the 18 no AAA patients with significant femoro-popliteal atherosclerotic disease. 
Figure 6.5 shows the number of AAA and no AAA significant distal disease patients with total scores $\geq 1, \geq 2$ and $\geq 3$ calculated by the new disease AAA detection program.

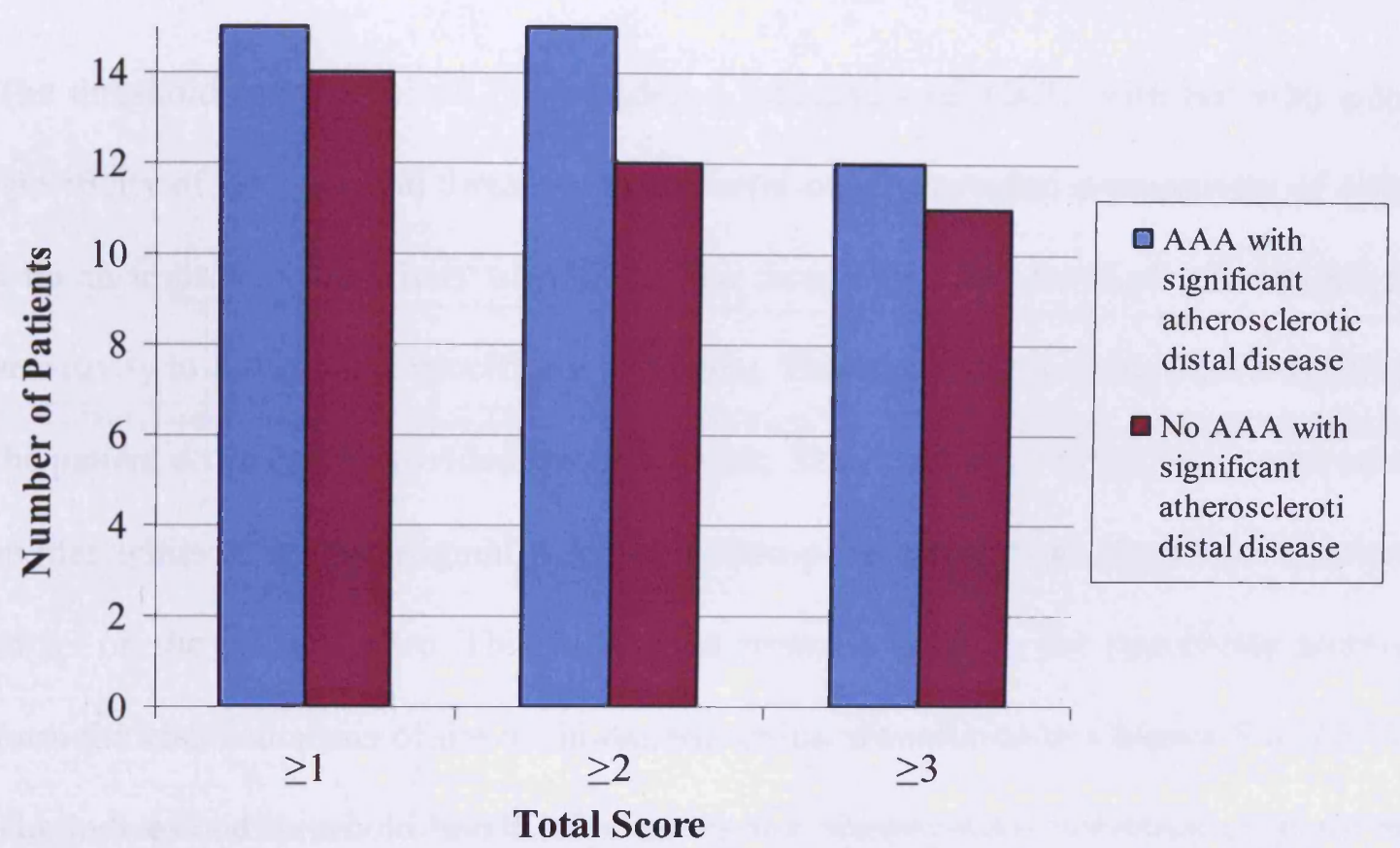

Figure 6.5: Number of AAA $(n=15)$ and no AAA $(n=18)$ patients with significant femoro-popliteal atherosclerotic disease with $\geq 1, \geq 2$ and $\geq 3$ features present.

\subsubsection{Disease AAA Detection Program Study groups' Results: Discussion}

The mean score per patient in the AAA group was 4.1 with a maximum score of 8 and a minimum score of 2 factors. The mean score per patient in the no AAA group was 2.9 with a maximum score of 8 and a minimum score of 0 . In comparison of these results to the results obtained from the original AAA detection program for the distal 
disease study groups, the mean scores in each group are lower. The mean score in the AAA group is reduced by 1.0 and the mean score in the no AAA group is reduced by 1.9. The difference between the mean scores of the two groups is increased from 0.3 to 1.9. However, this is still lower than the difference of 3.6 between the means of the AAA and normal patient groups with no significant atherosclerotic disease.

The threshold score level of $\geq 1$ provided a sensitivity of $100 \%$ with but with a low specificity of $22.2 \%$. The threshold score level of $\geq 2$ provided a sensitivity of $100 \%$ with an improved specificity of $33.3 \%$. The threshold score level of $\geq 3$ reduced the sensitivity to $80 \%$ with a specificity of $38.9 \%$. Therefore, the threshold level of $\geq 2$ on the patient score again provided the best result. The specificity of $33.3 \%$ is equivalent to that achieved by the original AAA detection program with an increased threshold of $\geq 3$ on the patient score. This is a slight improvement on the specificity attained form the visual analysis of the distal disease group waveforms in Chapter 5 of $27.7 \%$. The indices and threshold levels selected for the disease AAA detection program are probably more applicable to the distal disease groups than those in the original AAA detection program. 


\subsection{Response of Disease AAA Detection Program to Blind Test Groups}

The performance of the new disease AAA detection program was tested by subjecting the waveforms from the $3 \mathrm{AAA}$ and 4 no AAA patients with significant distal disease in the blind test groups that were separated at the start of the study (summarised in Table A3.2, Appendix 3 and A4.2, Appendix 4). The 5 indices are plotted for the left and right CFA waveforms for the AAA and no AAA test group patients in Figures 6.6a to $6.6 \mathrm{f}$. The dotted lines indicate the threshold levels set by the disease AAA detection program.

For the Maximum Systolic Down-Stroke Bandwidth Index, 1 waveform in each group had an index level above the threshold of 0.80 . For the Systolic Spike Index, 3 waveforms in each group had an index level above the threshold of 0.5. For the Reverse to Systolic Height Ratio Index, 1 of the AAA waveforms and none of the no AAA waveforms lay above the threshold level of 4.2. For the Integral Reverse Power Under Systolic Upstroke Index 1 AAA waveform and 2 no AAA waveforms had an Index level above the threshold of 0.10 . For the Integral Reverse Power Under Systolic Down-Stroke Index none of the AAA waveforms and 3 of the no AAA waveforms had an index level above the threshold of 1.25 . 


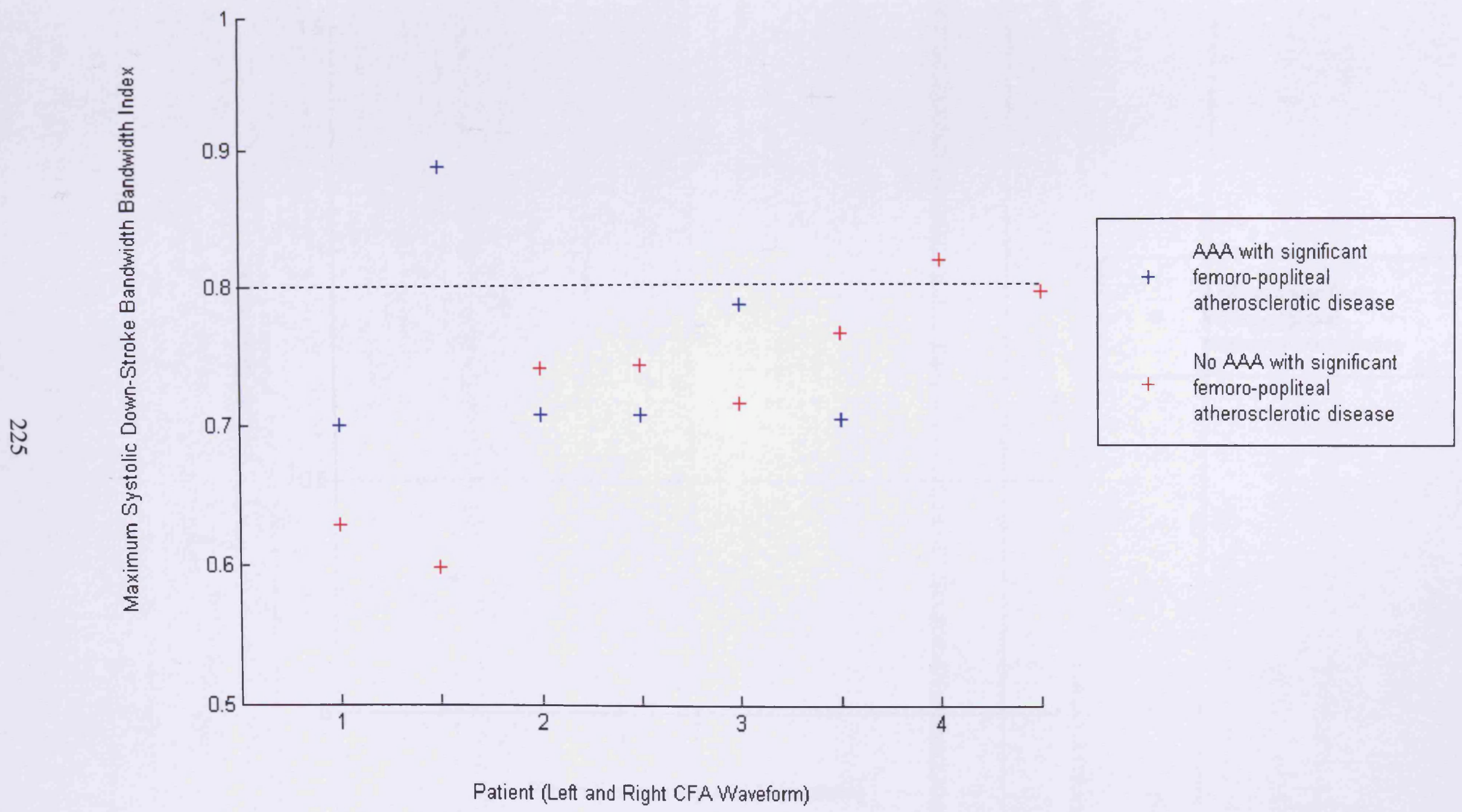

Figure 6.6a: Maximum Systolic Down-Stroke Bandwidth Index plotted for the left and right CFA waveforms from the 3 AAA and 4 normal patients in the distal disease test groups. 


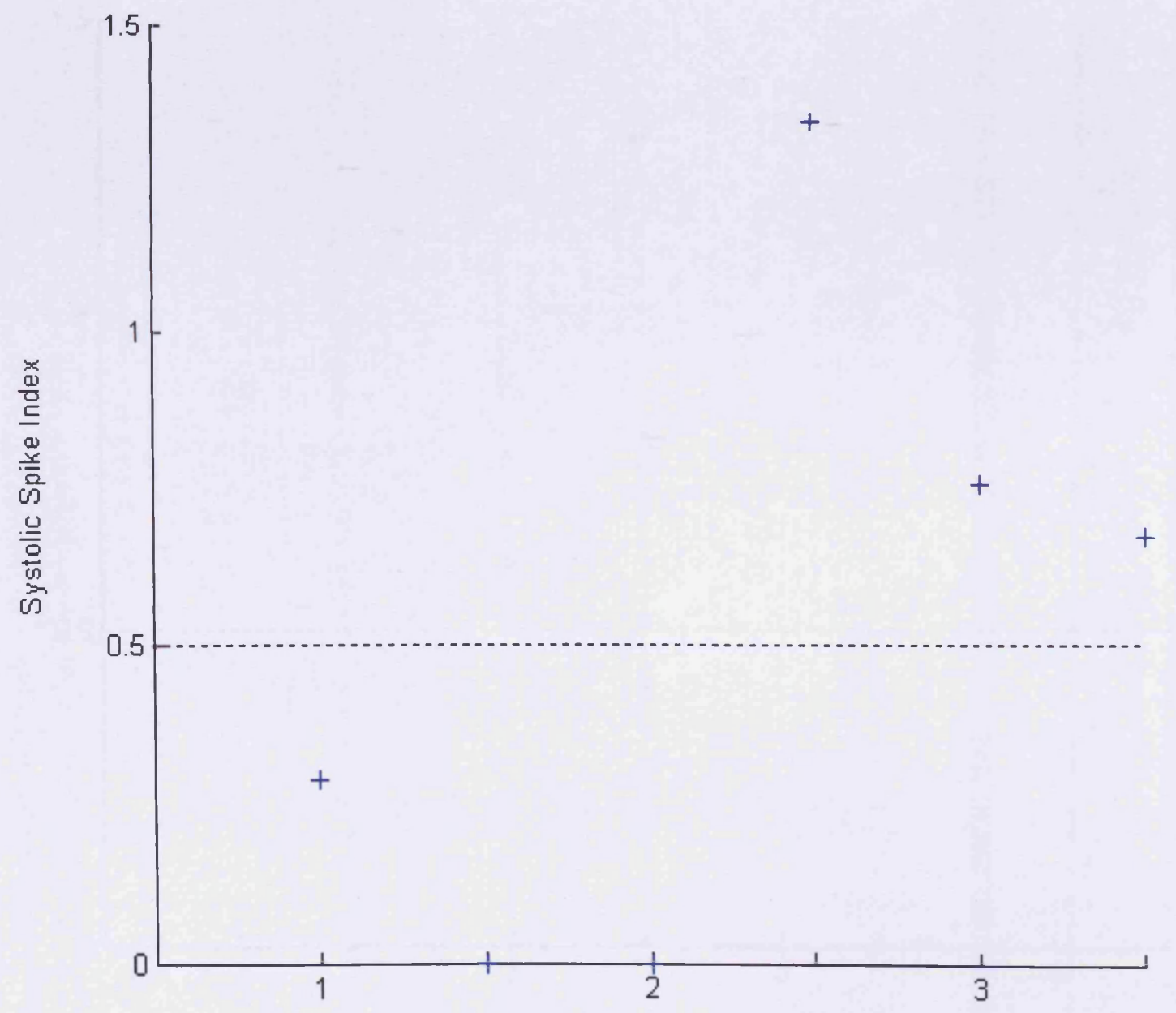

Patient (Left and Right CFA Waveform)

Figure 6.6b: Systolic Spike Index plotted for the left and right CFA waveforms from the 3 AAA patients in the distal disease test group. 


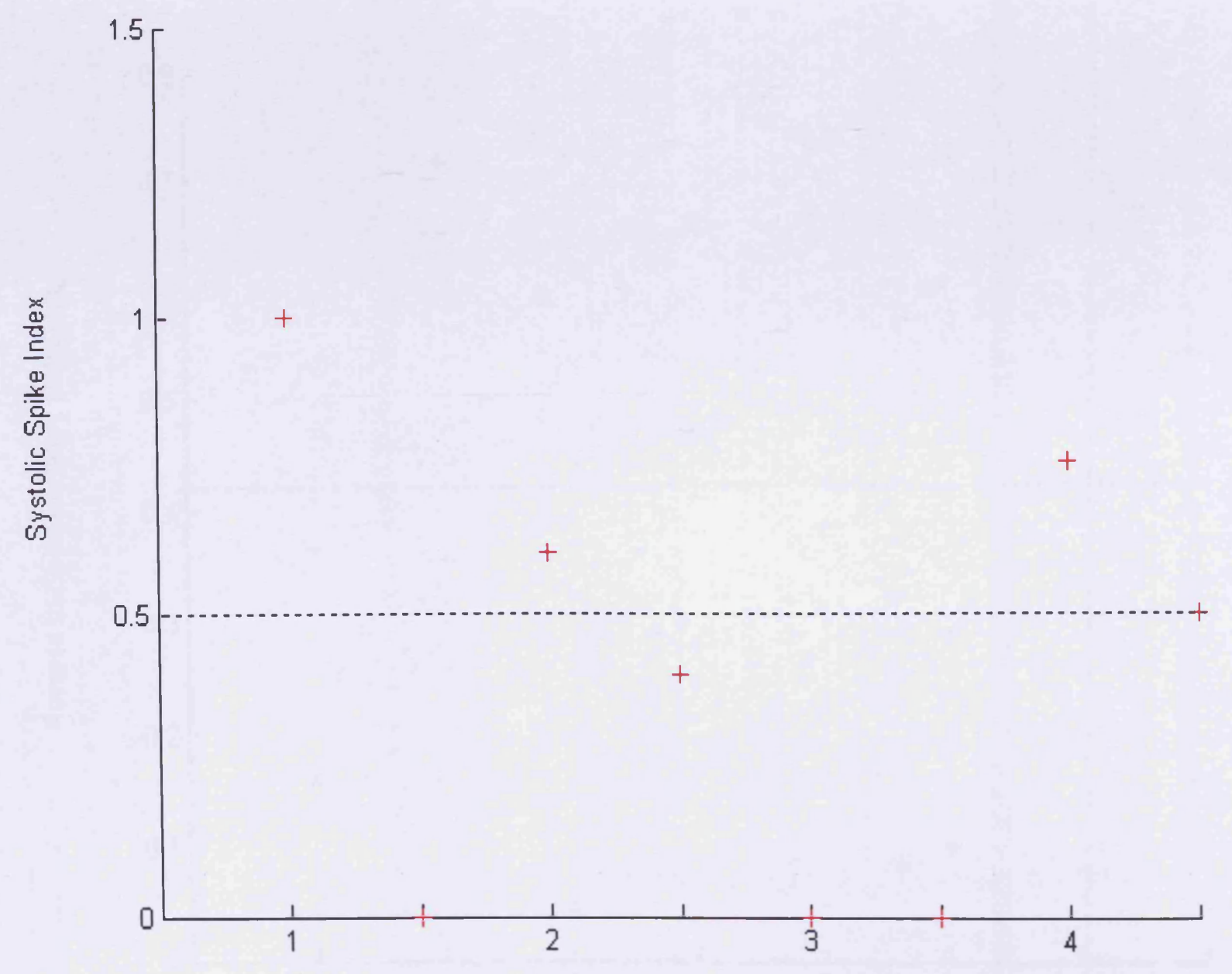

Patient (Left and Right CFA Waveform)

Figure 6.6c: Systolic Spike Index plotted for the left and right CFA waveforms from the 4 normal patients in the distal disease test group. 


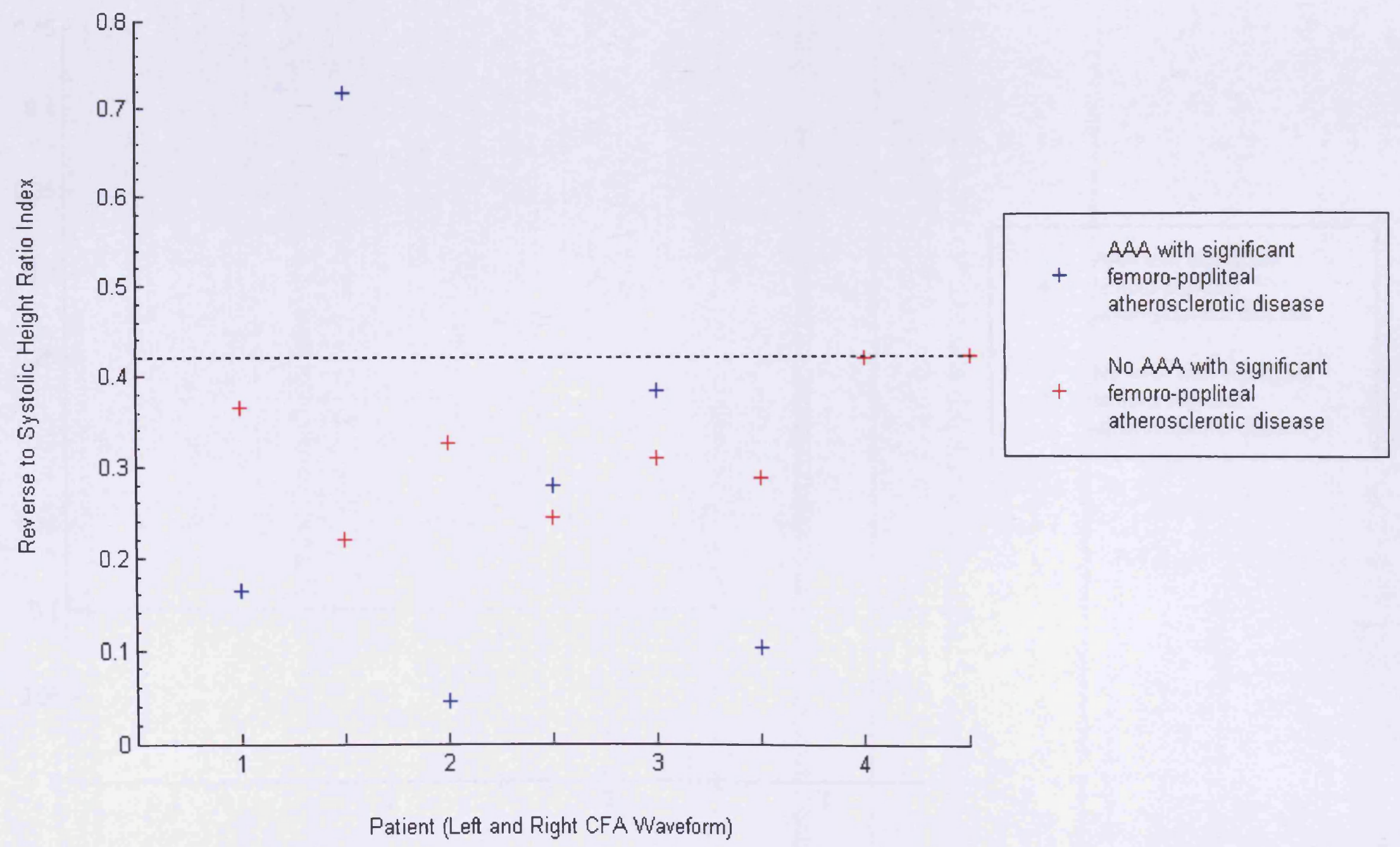

Figure 6.6d: Reverse to Systolic Height Ratio Index plotted for the left and right CFA waveforms from the 3 AAA and 4 normal patients in the distal disease test groups. 


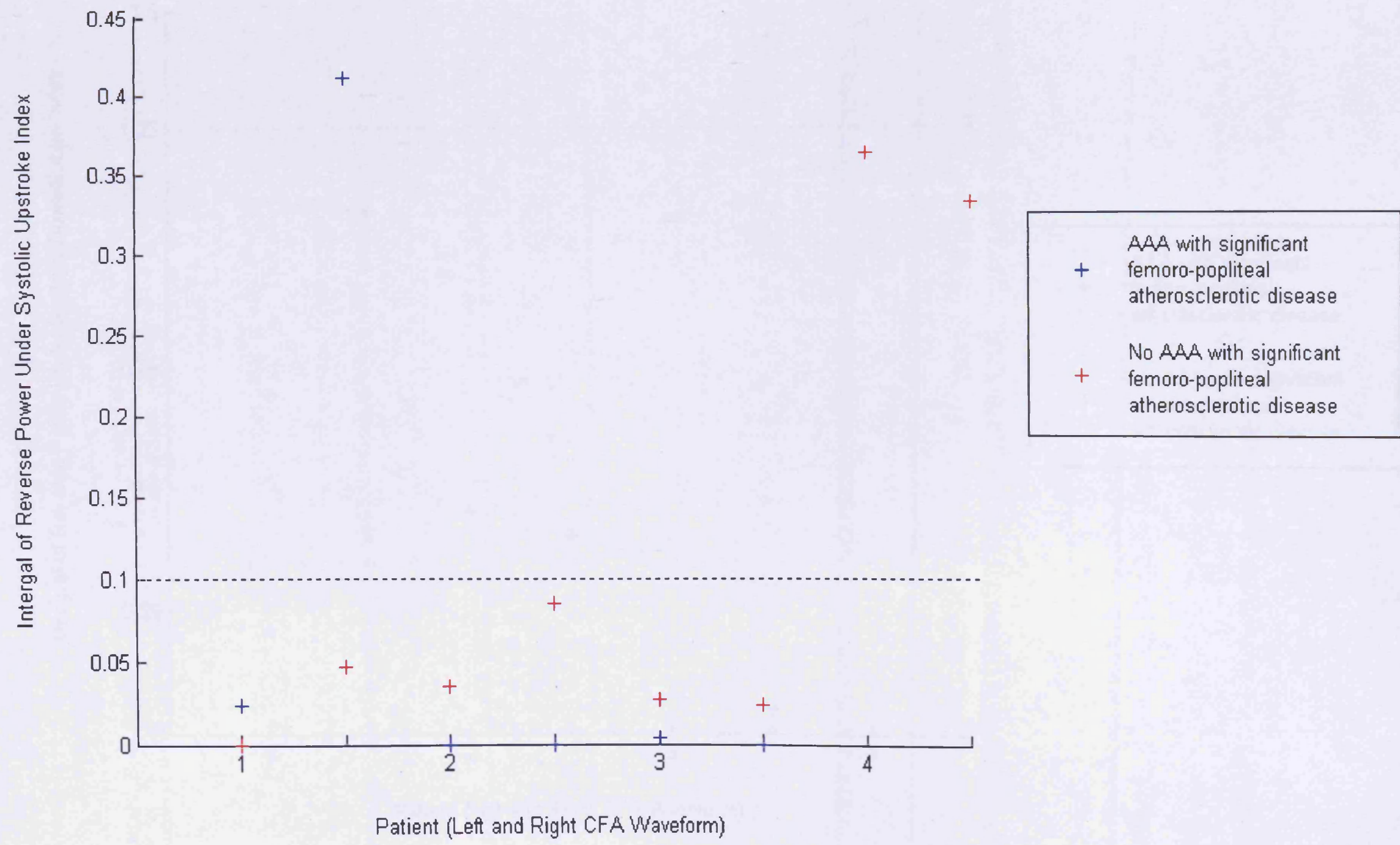

Figure 6.6e Integral of Reverse Power Under Systolic Upstroke Index plotted for the left and right CFA waveforms from the 3 AAA and 4 normal patients in the distal disease test groups. 

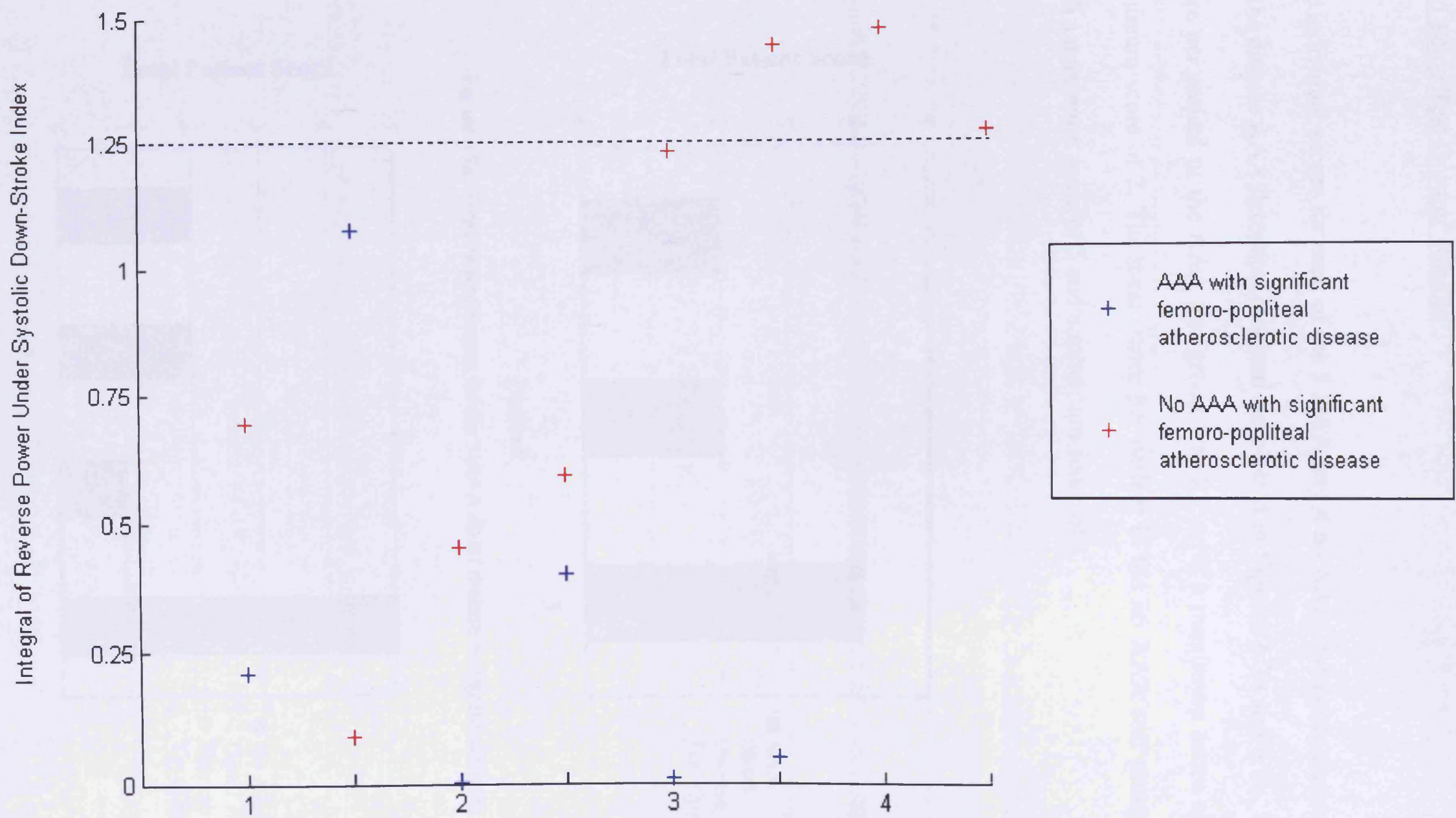

Patient (Left and Right CFA Waveform)

Figure 6.6f: Integral of Reverse Power Under Systolic Down-stroke Index plotted for the left and right CFA waveforms from the 3 AAA and 4 normal patients in the distal disease test groups. 


\section{$\underline{\text { 6.5.1 Blind Test Groups' Results }}$}

The individual scores for each of the 3 AAA and 4 no AAA test group patients output by the disease AAA detection program are plotted in Figures $6.7 \mathrm{a}$ and $6.7 \mathrm{~b}$. The mean score per patient in the AAA test group was 2.7 with a maximum score of 3 and a

minimum score of 2 . The mean score per patient in the no AAA test group was 3.3 with a maximum score of 5 and a minimum score of 1 .

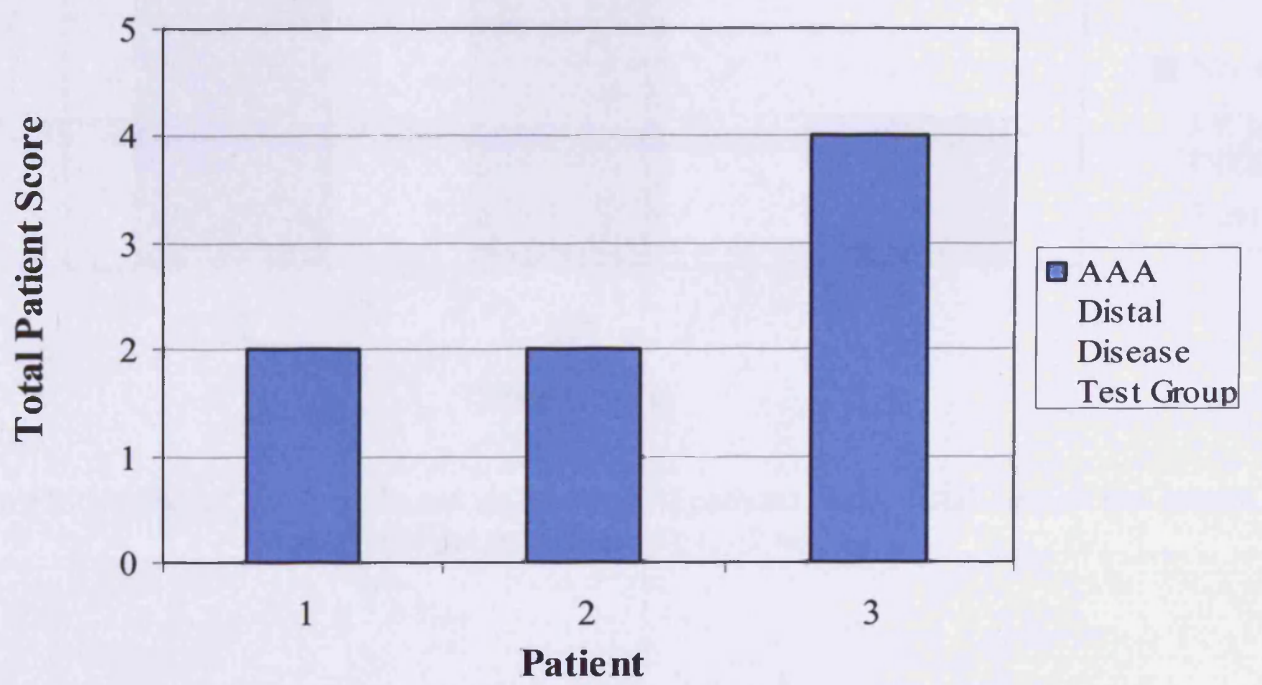

Figure 6.7a: Total Patient Scores for the 3 AAA distal disease test group patients

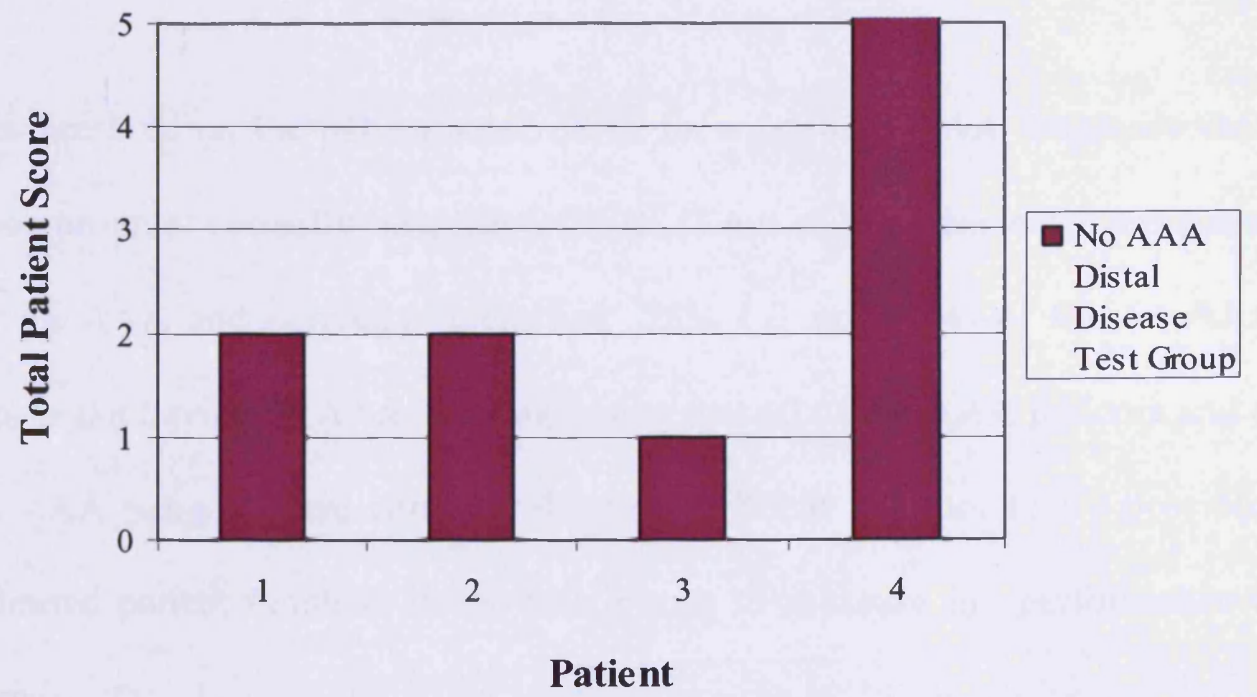

Figure 6.7b: Total Patient Scores for the 4 no AAA distal disease test group patients 
The number of test patients with total patient scores of $\geq 1, \geq 2$ and $\geq 3$ are plotted in Figure 6.8 .

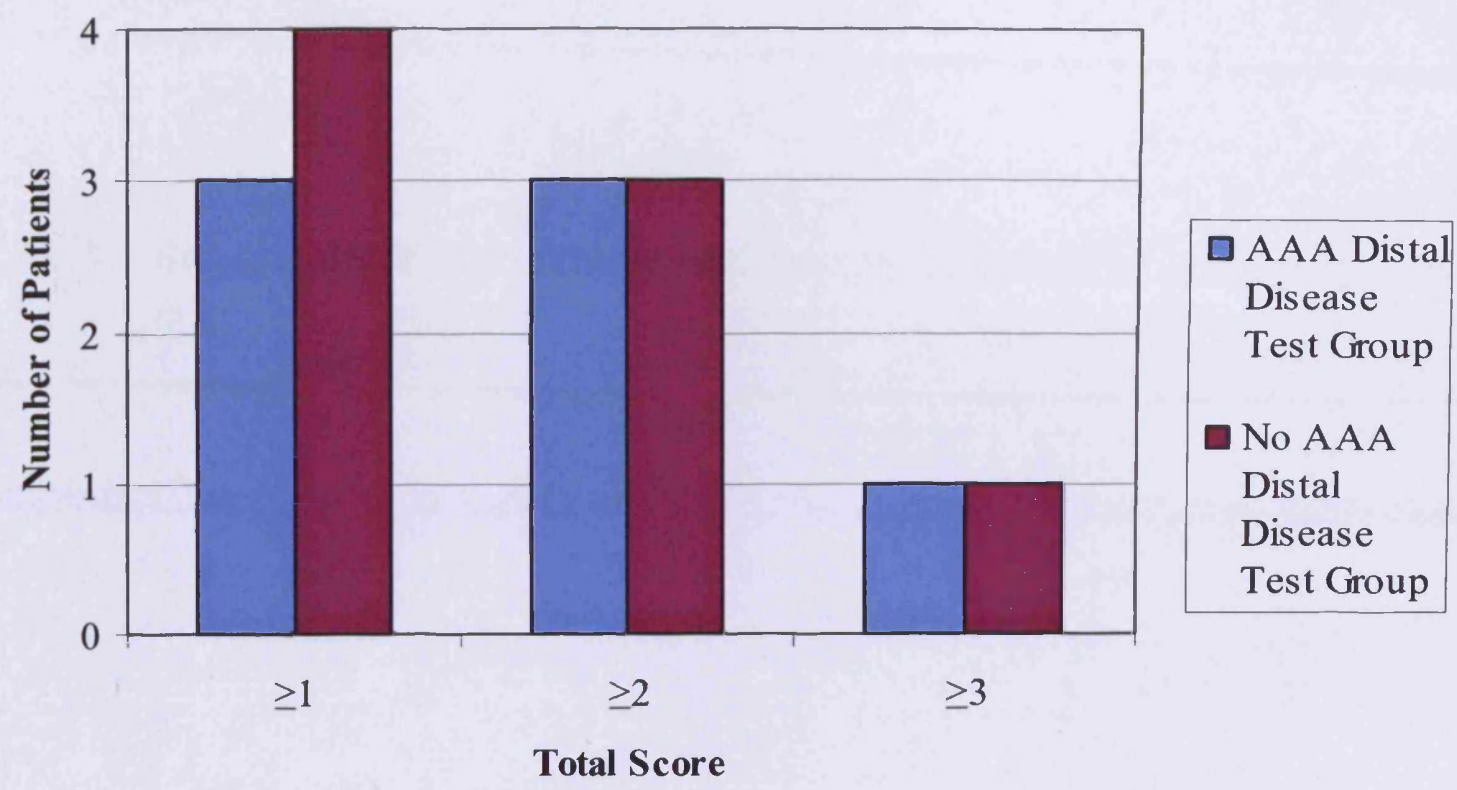

Figure 6.8: Number of AAA $(n=3)$ and no AAA $(n=4)$ patients in the distal disease test groups with total patient scores of $\geq 1, \geq 2$ and $\geq 3$

\subsubsection{Blind Test Groups' Results: Discussion}

For the threshold on the patient score of $\geq 2$ for a positive AAA diagnosis the AAA detection program correctly 'identified' $100 \%$ ( 3 out of 3 ) of the test AAA patients as having an AAA and correctly 'identified' $75 \%$ ( 3 out of 4 ) of the no AAA test patients as not having an AAA. It is promising that all of the AAA patients and one of the no AAA patients were correctly diagnosed, but it was not really possible with such limited patient numbers in the test groups to measure the performance of the program. 


\subsection{AAA Detection Program for Patients with Significant Iliac Atherosclerotic}

\section{Disease}

The visual analysis of the CFA waveforms in Chapter 5 showed that the 5 features present in the no significant atherosclerotic disease and significant femoro-popliteal atherosclerotic disease groups were almost completely removed by the presence of significant atherosclerotic iliac (proximal) disease. Visual identification of these features for the 6 AAA and 7 no AAA patients with significant proximal disease did not provide any separation between the AAA and no AAA patients for a sensitivity of $100 \%$. In order to test the performance of the AAA detection program for patients with significant proximal atherosclerotic disease the CFA waveforms from the 6 AAA and 7 no AAA patients in the study groups were subjected to the disease AAA detection program.

Unfortunately, the AAA and disease AAA detection programs did not run for the waveforms with proximal disease because the pulse foot at the end of the systolic peak could not be located with the criteria set by the program. This was due to the fact that the waveforms were very damped. However, because the features which seemed to be characteristic to AAA were almost completely removed from the CFA waveforms in the presence of significant proximal disease in the visual inspections, it was decided to let the program 'break' if the pulse foot of the systolic down-stroke could not be located. If this system was implemented as a screening tool, this would indicate the need for further investigation of the patient. Further investigation of the disease state of the aorto-iliac and femoro-popliteal arteries would be necessary regardless of whether the patient had an AAA. 
From experience in the Doppler Ultrasound department at UHW, approximately $25 \%$ of patients with significant lower limb arterial disease have significant proximal disease. Studies indicate the prevalence of significant lower limb PAD in the general population is around $12 \%$. From the estimate of $25 \%$ for the prevalence of significant proximal disease, this would only involve around $3 \%$ of the general population. Therefore, because the proportion of the population with significant aorto-iliac disease is very low, the inability of the disease detection program to function for these patients may not be critical to the overall performance of the program as a screening tool.

\subsection{Discussion}

In this chapter, two different MATLAB programs were tested on patients with and without AAA with significant femoro-popliteal atherosclerotic disease. The aim was to determine whether AAA could be detected in these patients by automatic, objective analysis of the CFA waveforms. The additional changes to the CFA waveforms set up in the presence of significant femoro-popliteal atherosclerotic disease made it much more difficult to discriminate between patients with and without AAA, even with an objective analysis method. The original AAA detection program, with a threshold of $\geq 2$ placed on the total patient score, provided a sensitivity of $100 \%$ and a specificity of only $11.1 \%$ for AAA detection. The specificity was increased to $33.3 \%$ with an increased threshold of $\geq 3$ placed on the total patient score. Inspection of the plots of the 6 indices showed that the reverse power density index was unsuitable and that the discrimination between the groups by the five remaining indices could be improved by adjustment of the set threshold levels. 
The new 'disease AAA detection program' combined the 5 indices: 1) Maximum Systolic Down-Stroke Bandwidth Index, 2) Systolic Spike Index, 3) Reverse to Systolic Height Ratio Index, 4) Integral Reverse Power Under Systolic Upstroke Index and 5) Integral Reverse Power Under Systolic Down-Stroke Index with adjusted threshold levels. This program achieved a sensitivity of $100 \%$ and a specificity of $33.3 \%$ for AAA detection in patients with significant femoro-popliteal atherosclerotic disease. This improved on the specificity of $11.1 \%$ achieved by the original AAA detection program, and marginally improved on the specificity level of 27.7\% achieved in the visual inspection of the waveforms in Chapter 5. The indices and threshold levels implemented by the disease AAA detection program were also more applicable to the waveforms in the distal disease patient groups.

If the AAA detection programs were to be implemented as a method for screening for AAA, it may be possible to combine the use of the 2 programs with a technique such as an ABPI measurement to assess whether the patient has significant PAD. If the ABPI measurement was normal, the original program could be implemented to analyse the CFA waveforms. If the ABPI measurement indicated significant disease, the Disease AAA program described in this chapter could be implemented.

The relatively poor specificity achieved for patients with significant atherosclerotic disease may not be critical to the overall performance of the combined screening method. If these results are projected onto the general population with no significant aorto-iliac or femoro-popliteal occlusive arterial disease, this gives a sensitivity of $100 \%$ and specificity of around $73 \%$ for $88 \%$ of the general population, assuming the prevalence of lower limb PAD is around $12 \%$. 
The screening studies for AAA indicate that a single scan would be performed on all males of 65 years old. PAD is a progressive disease, and studies indicate that the prevalence of PAD is around 7\% for those aged $60-69$ years old and around $23 \%$ for those aged over 80 years old (Fowkes, Housley et al. 1991; Ostchega, Paulose-Ram et al. 2007). Therefore, if this AAA detection technique was applied as an AAA screening technique in 65 year olds, it would provide a sensitivity of around $100 \%$ and specificity of around $70 \%$ for $93 \%$ of the screened population. In the remaining $7 \%$ of the screened population with significant atherosclerotic arterial disease, around $25 \%(1.75 \%$ of the screened population) would have significant proximal disease and would automatically require further investigation of the aorto-iliac and femoropopliteal arteries. For the remaining $5.25 \%$ of the screened population with significant femoro-poplital disease, the disease AAA detection program would provide a sensitivity of around $100 \%$ with a specificity of around $33 \%$. If the original AAA detection program was used for the population with no significant disease and the disease AAA detection program was used for the population with significant atherosclerotic disease, combining the outcomes for the no significant disease, distal and proximal disease groups gives an overall sensitivity of around $100 \%$ and an overall specificity of around $70 \%$ to AAA detection in the screened population.

If the outcomes for the original AAA detection program (with a threshold on the total patient score of $\geq 2$ ) are projected onto the screened population, the sensitivity to AAA would be very high (around 100\%). The specificity for the $93 \%$ of the screened population without significant atherosclerotic disease would be around $73 \%$. The specificity for the small proportion $(\sim 7 \%)$ of the population with significant 
atherosclerotic disease would only be around $11 \%$. Hence, the overall specificity for the screened population would be around $68 \%$.

Further work would be necessary to determine the feasibility of implementing the two AAA detection programs in conjunction with a simple method for arterial disease detection such as an ABPI measurement. Although the combined method may provide a slightly better specificity to AAA in the screened population than the original AAA detection program. For a practical view point, the implementation of the single program would be much simpler and because such a small percentage $(\sim 7 \%)$ of the screened population would have significant PAD, the reduction of around $2 \%$ in specificity may not be critical to the overall performance of the device in a screening programme.

The sensitivity and specificity levels provided by both the combined and single AAA detection programs are adequate for a tool to screen for AAA and indicate that objective analysis of CFA waveform features is a promising, alternative technique to conventional ultrasound for AAA detection. 
Chapter 7: Characterisation of AAA In Patients with No Significant Atherosclerotic Arterial Disease

\subsection{Introduction and Aim}

The AAA modelling studies reviewed in Chapter 1 indicated that disturbed and possibly turbulent flows occur within AAAs, and that these disturbances may be translated distally. The primary aim of this research was to develop a method to detect AAA by analysis of the CFA waveform. The MATLAB based AAA detection program described in Chapter 4 was capable of detecting AAA with $100 \%$ sensitivity and around $70 \%$ specificity for patients without significant iliac or femoro-popliteal atherosclerotic arterial disease. The modelling studies also indicated that the flow disturbances set up within AAA models are dependant upon the AAA size and shape. The aim of the work described in this chapter was to determine whether, in addition to detecting AAA, the information provided by the AAA detection program could also be used to provide information on the physical characteristics of the AAA such as the AAA size and the presence of ILT. This information is useful to the clinician when determining the optimum management path and treatment options. 


\subsection{Method}

The cohort for this section of work was the group of 37 patients with AAA but no significant atherosclerotic arterial disease (30 patients in the study group, plus the 7 patients in the test group).

In the first section of the work, the AAA size and presence of intra-luminal thrombus were plotted against the total scores for each patient calculated by the AAA Detection Program. Linear regression analysis was applied to determine whether there was any correlation between the AAA size and the presence of ILT with the program results.

The aim of the second section of the work was to investigate whether there was any correlation between each of the 6 individual indices: 1) Maximum Systolic DownStroke Bandwidth Index, 2) Systolic Spike Index, 3) Reverse to Systolic Height Ratio Index, 4) Reverse Power Density Index, 5) Integral Reverse Power Under Systolic Upstroke Index and 6) Integral Reverse Power Under Systolic Down-Sstroke Index calculated by the AAA detection program and AAA size. The MATLAB code in the AAA detection program was altered so that the levels for the 6 individual indices calculated for the right and left CFA waveforms for each patient were output before the weighting factors or thresholds were applied. The mean and maximum index levels for each patient were plotted against AAA size and linear regression analysis was applied to determine whether they provided any correlation. 


\section{$\underline{7.3 \text { Results }}$}

\subsubsection{Investigation of Total Patient Scores with AAA Size and ILT}

The total patient scores for the 37 patients were plotted against AAA size as in Figure 7.1. It can be seen that there is no significant correlation between the total patient score and the AAA size $\left(\mathrm{R}^{2}=0.002, \mathrm{P}=0.787\right)$.

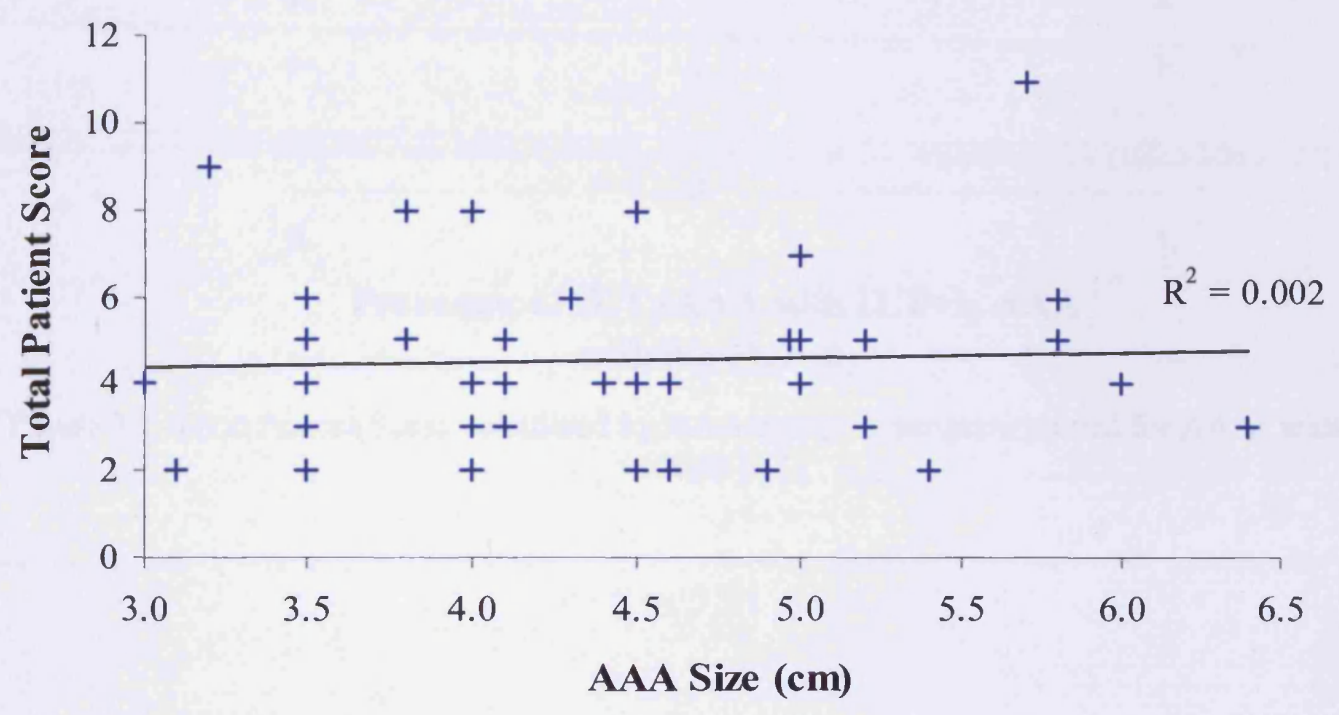

Figure 7.1: Total Patient Score calculated by the AAA detection program plotted against AAA Size.

The AAA sizes for AAAs with and without ILT are plotted in Figure 7.2. It can be seen that there is also no real difference in the total patient scores for AAAs with and without ILT. 


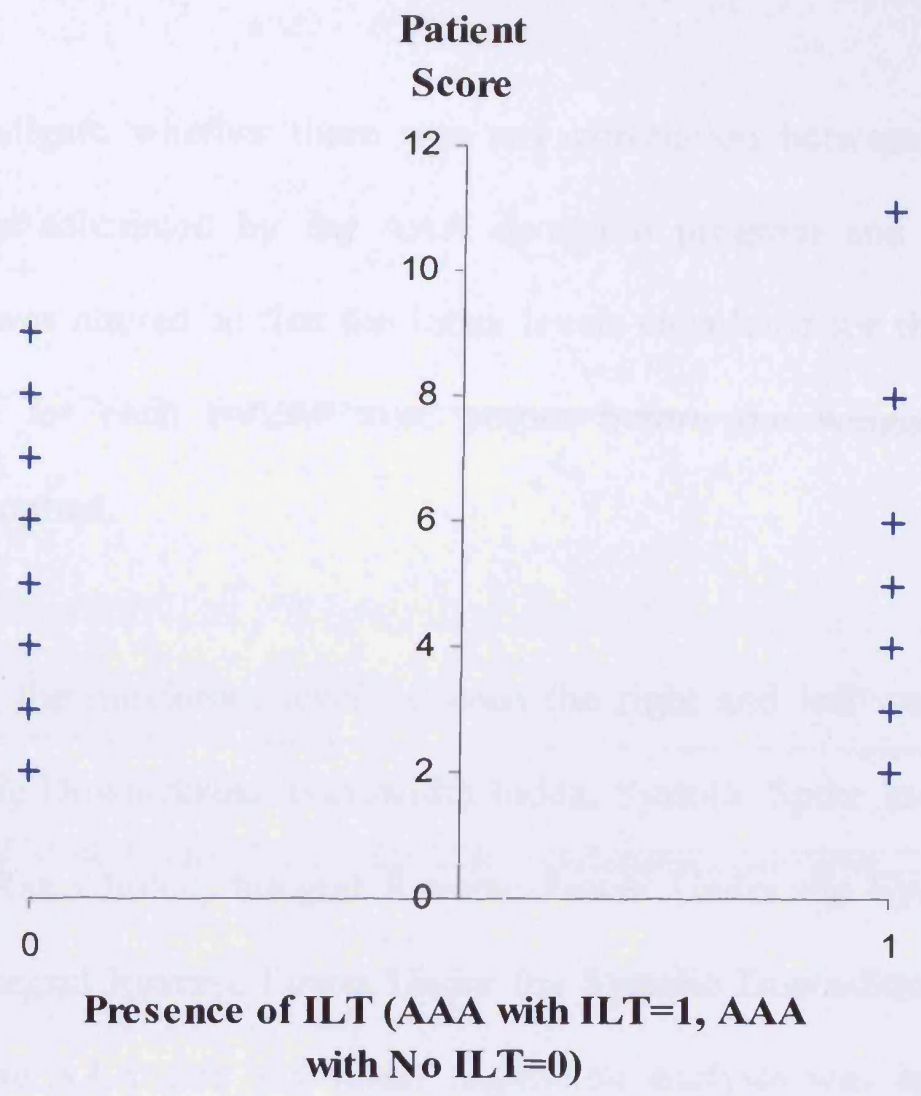

Figure 7.2: Total Patient Score calculated by AAA detection program plotted for AAAs with and without ILT.

The indices and threshold levels used in the AAA detection program were selected to optimise the discrimination between AAA and normal patients to provide a method to detect AAA, not and not specifically to characterise the size or ILT status. Therefore, it may be possible that a different selection of indices and threshold levels may provide better characterisation of AAA size and ILT. 


\subsubsection{Investigation of Individual Index Scores for AAA Size Characterisation}

In order to investigate whether there was any correlation between each of the 6 individual indices calculated by the AAA detection program and AAA size, the MATLAB code was altered so that the index levels calculated for the right and left CFA waveforms for each patient were output before the weighting factors or thresholds were applied.

For each patient, the maximum level between the right and left waveforms of the Maximum Systolic Down-Stroke Bandwidth Index, Systolic Spike Index, Reverse to Systolic Height Ratio Index, Integral Reverse Power Under the Systolic Upstroke Index and the Integral Reverse Power Under the Systolic Down-Stroke Index were plotted against the AAA size and linear regression analysis was applied. For the Reverse Power Density Index the minimum level between the right and left waveform values were plotted against AAA size. The plots for the 6 indices are given in Figures 7.3 to 7.8 .

On visual inspection of the graphs and application of linear regression analysis, there was no significant correlation between the AAA size and the maximum out of the values for the right and left waveforms for the Systolic Down-Stroke Bandwidth Index $\left(\mathrm{R}^{2}=0.000, \mathrm{P}=0.952\right)$, Systolic Spike Index $\left(\mathrm{R}^{2}=0.001, \mathrm{P}=0.876\right)$ and the Integral Reverse Power Under Systolic Down-Stroke Index $\left(\mathrm{R}^{2}=0.003, \mathrm{P}=0.740\right)$. There is some weak correlation that is statistically significant between the maximum of the Maximum Reverse to Systolic Height Ratio Index and AAA size plotted in Figure 7.5. For this index, there is a slight positive trend $\left(\mathrm{R}^{2}=0.171, \mathrm{P}=0.011\right)$ with 
AAA size. There is also weak correlation that approaches statistical significance between AAA size and the minimum Reverse to Power Density Index plotted in Figure 7.6 $\left(\mathrm{R}^{2}=0.082, \mathrm{P}=0.086\right)$, and the Integral Reverse Power Under Systolic Upstroke Index $\left(\mathrm{R}^{2}=0.080, \mathrm{P}=0.091\right)$. For the Reverse Power Density Index, there is a possible negative trend with AAA size and for the Integral Reverse Power Under the Systolic Upstroke Index there is a possible positive trend with AAA size.

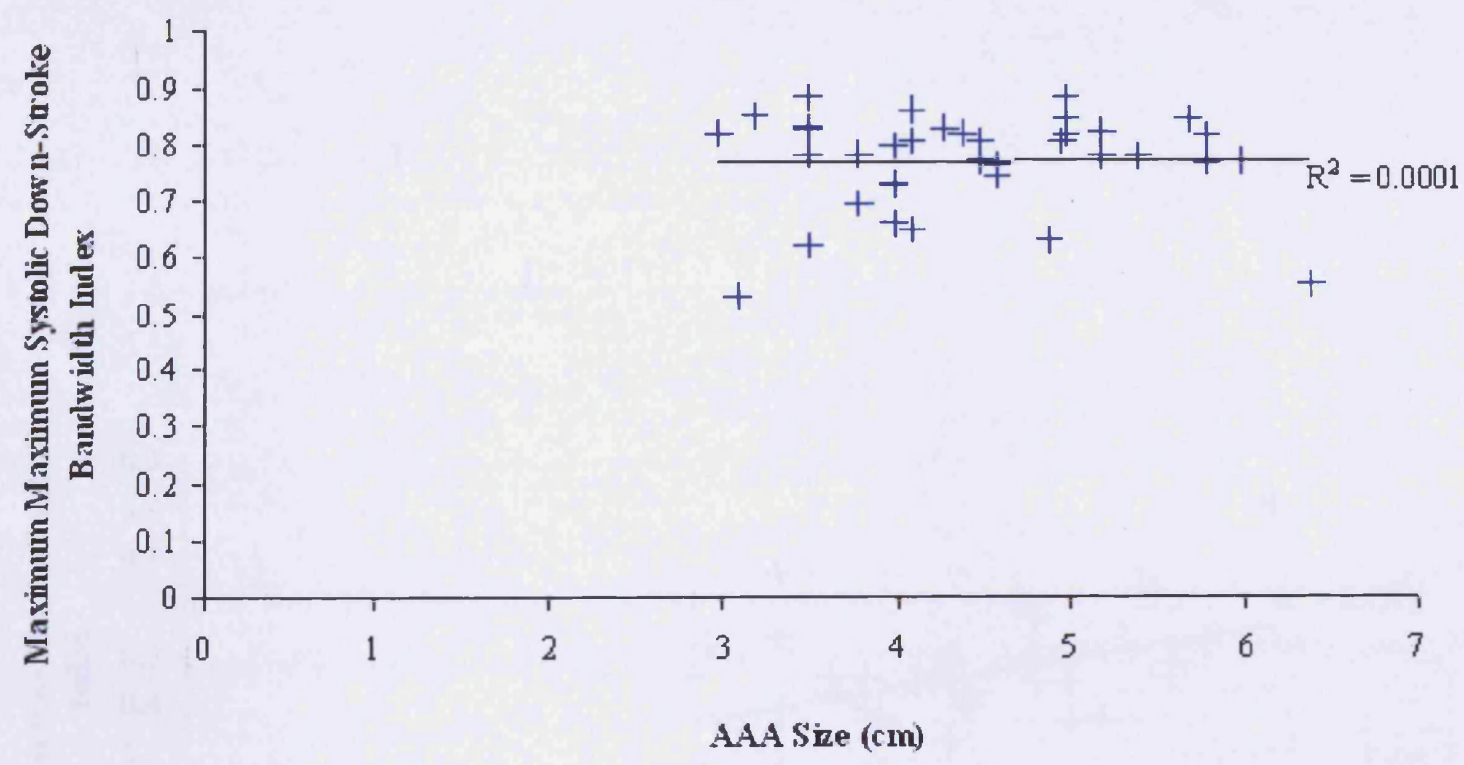

Figure 7.3: Maximum Maximum Systolic Down-Stroke Bandwidth Index for patient plotted against AAA size. 


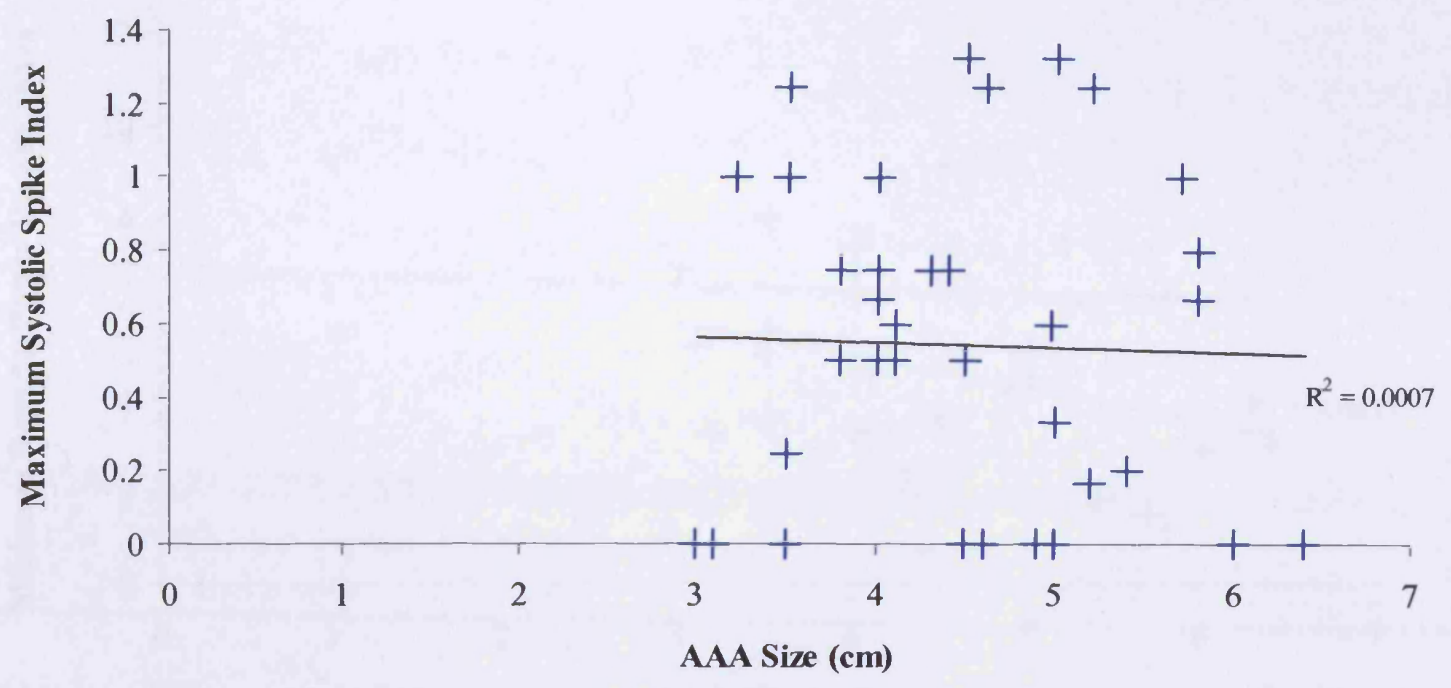

Figure 7.4: Maximum Systolic Spike Index for patient plotted against AAA size.

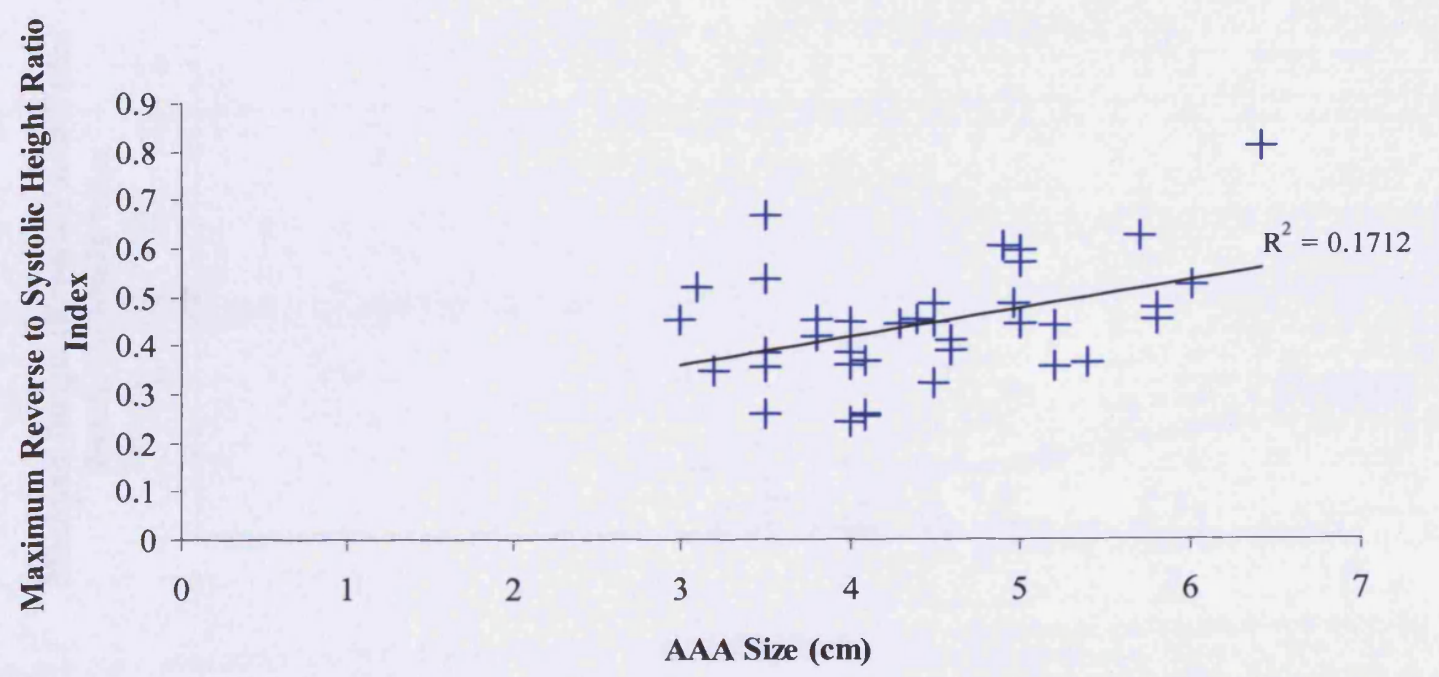

Figure 7.5: Maximum Reverse to Systolic Height Ratio Index for patient plotted against AAA size. 


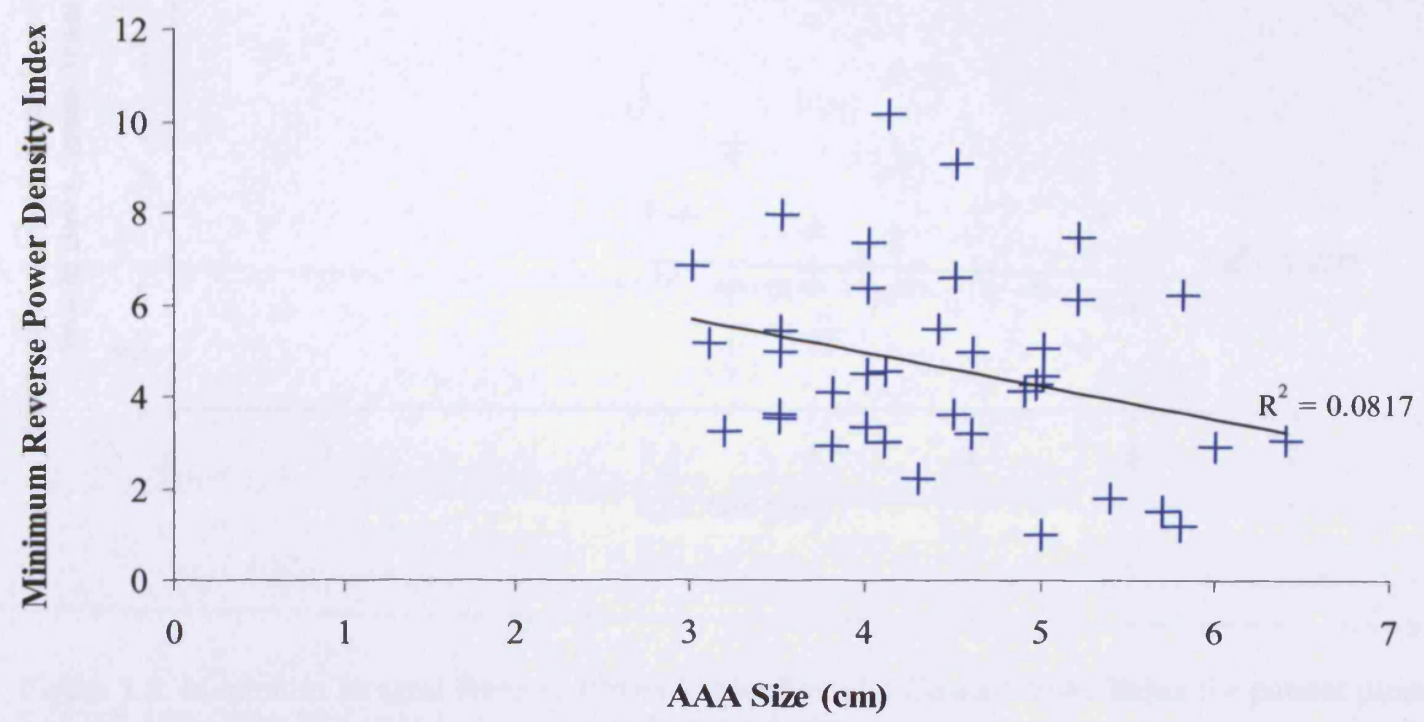

Figure 7.6: Minimum Reverse Power Density Index for patient plotted against AAA size.

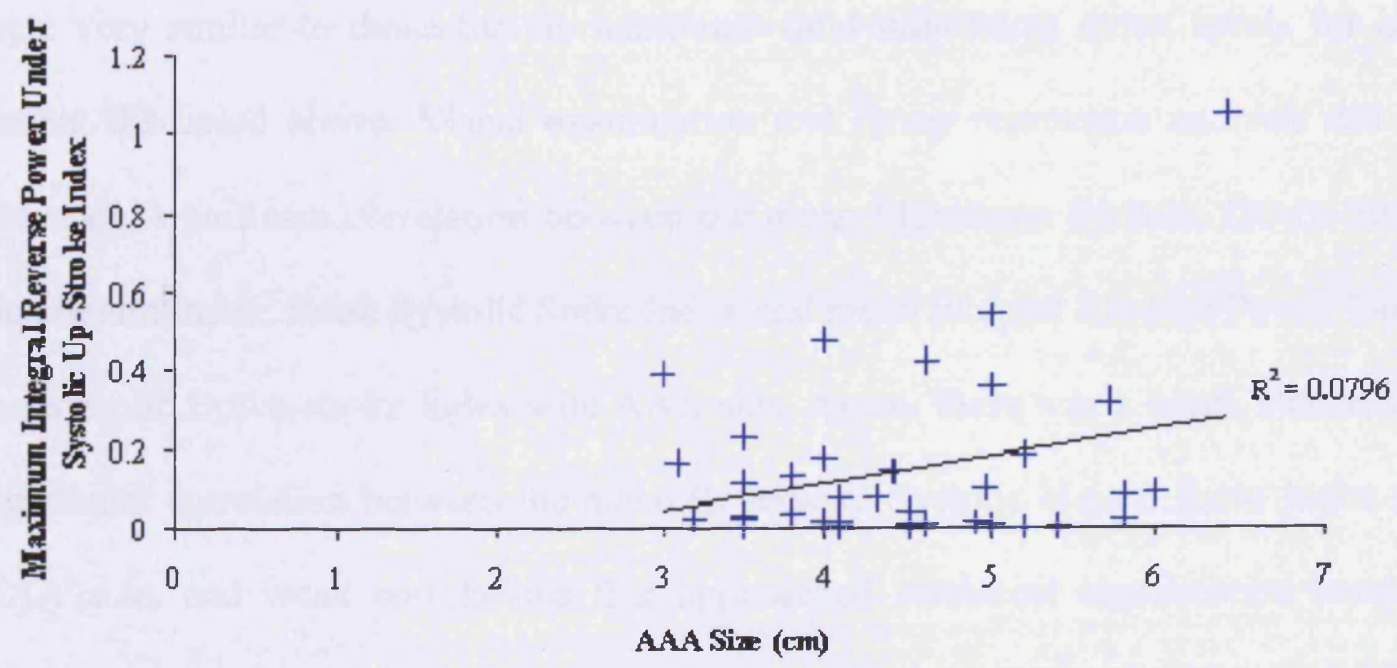

Figure 7.7: Maximum Integral Reverse Power Under Systolic Upstroke index for patient plotted against AAA size. 


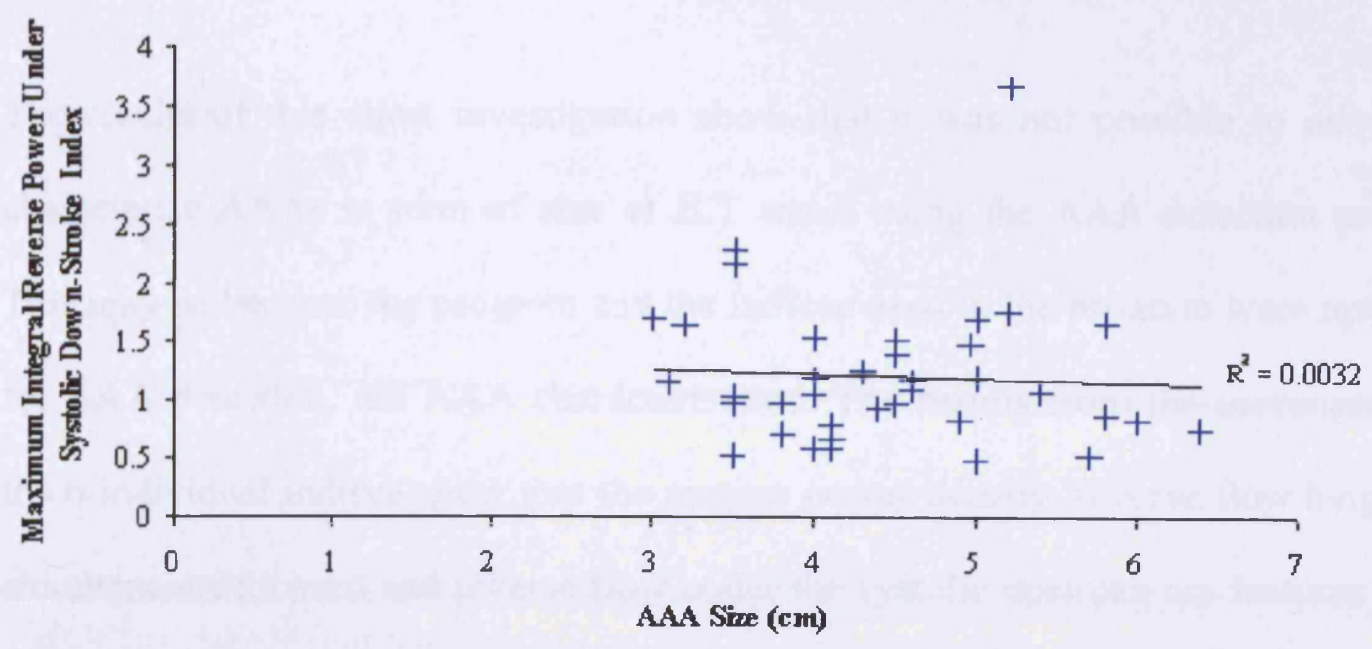

Figure 7.8: Maximum Integral Reverse Power Under Systolic Down-Stroke Index for patient plotted against AAA size.

For each patient, the mean of the index values for the right and left CFA waveforms were calculated for each of the 6 indices and plotted against AAA size. The results were very similar to those for the maximum (and minimum) index levels for each patient discussed above. Visual examination and linear regression analysis did not show any significant correlation between the mean Maximum Systolic Down-Stroke Bandwidth Index, mean Systolic Spike Index and mean Integral Reverse Power Under the Systolic Down-stroke Index with AAA size. Again, there was a weak, statistically significant correlation between the mean Reverse to Systolic Height Ratio Index and AAA size, and weak correlations that approached statistical significance between AAA size and the mean Integral Reverse Power Under the Systolic Upstroke Index and the mean Reverse Power Density Index. 


\subsection{Discussion}

The results of this short investigation show that it was not possible to adequately characterise AAAs in term of size or ILT status using the AAA detection program. This may be because the program and the indices used in the program were optimised for AAA detection, not AAA characterisation. The results from the investigation of the 6 individual indices show that the reverse power density, reverse flow height and simultaneous forward and reverse flow under the systolic upstroke are features within the CFA waveform which may be useful for characterising AAA size. It may be possible to characterise the physical aspects of an AAA from the CFA waveform using a program optimised for the characterisation of AAA, possibly involving different analysis methods. In order to characterise AAA it is first necessary to be able to detect AAA, and most important result of the overall research project is that it is possible to detect AAA by analysis of the CFA waveform. 


\section{Chapter 8: Summary and Conclusions}

\subsection{Research Summary}

The main aim of this research project was to determine whether aneurysms of the abdominal aorta can be detected by analysis of the CFA Doppler waveform. Visual analysis of CFA waveforms from patients with no significant atherosclerotic disease of the iliac or femoro-popliteal arteries, discussed in Chapter 3, indicated that there are certain changes to the normal CFA Doppler waveform in the presence of AAA. There were 5 features noted, that were present predominantly in the waveforms from AAA patients. These were: 1) spectral broadening on the systolic down-stroke, 2) the appearance of spikes on the systolic down-stroke, 3) an irregular reverse flow pattern, 4) simultaneous forward and reverse flow during systole and 5) elongation of the waveform during the cardiac cycle. It was possible to detect AAA by a method based on visual identification of these features for 30 patients with AAA and 30 patients without AAA with a sensitivity of $93.3 \%$ and a specificity of $70 \%$. These results indicated that it may be possible to detect AAA by feature analysis of the CFA Doppler waveform. Visual analysis of Doppler waveforms is subjective and requires a high level of training; therefore a more objective, automated analysis method was necessary. A MATLAB based computer program was written to automatically perform the CFA waveform analysis and determine whether or not an AAA was present. The CFA waveform Doppler waveform is a 2-dimensional representation of a 3- dimensional data set of frequency, amplitude and time. Because the CFA Doppler waveform is so complex, a number of simplified vectors were calculated from the waveforms to represent certain waveform parameters, for example, the maximum 
frequency envelope and an envelope to represent band-width. These vectors were then used to calculate a number of different indices to provide a measure for different aspects of the 5 visual waveform features. Plots of these indices were visually inspected to determine which indices provided the best level of separation between the AAA and normal patients. A MATLAB program was then written to combine these indices in different combinations, with a number of different threshold levels and weighting factors applied to determine the optimum combination to provide the highest levels of sensitivity and specificity for AAA detection. The optimum combination involved 6 indices to describe the 5 visual features: Maximum Systolic Down-Stroke Bandwidth Index, Systolic Spike Index, Reverse Power Density Index, Reverse to Systolic Height Ratio Index, Integral Reverse Power Under Systolic Upstroke Index and Integral Reverse Power Under Systolic Down-Stroke Index. The program calculated a score for each patient based on where the index values calculated for the right and left CFA waveforms lay in relation to set threshold levels. A threshold was then placed on the total patient scores to best separate the AAA and normal patients. The program produced very promising results with a sensitivity of $100 \%$ and a specificity of $73.3 \%$ for AAA detection in the 30 AAA and 30 normal patients without significant atherosclerotic disease. The program was then subjected to waveform data from blind test sets of waveform data, and produced a sensitivity of $100 \%$ and specificity of $63.6 \%$ for 7 AAA and 11 normal test patients. The program also achieved a sensitivity of $100 \%$ for AAA detection in 9 patients with AAA and additional aneurysmal disease of the iliac or popliteal arteries.

The process was then applied to waveform data from patients with and without AAA with significant atherosclerotic disease of the iliac and femoral-popliteal arteries. 
Visual and MATLAB based analysis of the waveforms in Chapters 5 and 6 did not provide as good differentiation between the AAA and normal patients. The 5 features noted visually in patients with AAA, but no significant atherosclerotic disease were also present in the waveforms from patients with significant femoro-popliteal disease. However, additional waveform features set up by the atherosclerotic disease made it much more difficult to separate the patients with and without AAA. For the 15 AAA and 18 no AAA patients with significant femoro-popliteal disease, AAA detection based on visual identification of the 5 waveform features provided a sensitivity of $100 \%$ but a specificity of only $27.7 \%$. Automatic, objective analysis was performed using two different MATLAB programs. The original MATLAB AAA detection program achieved a sensitivity of $100 \%$ but a very poor specificity of only $11.1 \%$. The disease group AAA detection program combined 5 out of the 6 indices with adjusted threshold levels and provided improved results with a sensitivity of $100 \%$ and a specificity of $33.3 \%$. For the 6 AAA and 7 no AAA patients with significant atherosclerotic disease of the iliac arteries, visual analysis of the waveforms indicated that almost all the waveform features were removed. The features that were present in the waveforms were more likely to be set up by the atherosclerotic arterial disease rather than AAA. All the CFA waveforms had very high levels of spectral broadening and AAA detection based on visual feature analysis of the waveforms provided $100 \%$ sensitivity but no specificity. The MATLAB programs did not function for these patients because the waveforms were very damped. It was therefore not possible to adequately discriminate between patients with and without AAA with significant disease of the iliac arteries by feature analysis of the CFA waveform. 
A secondary aim of the research was to determine whether, in addition to detecting AAA, the information provided by the AAA detection program could also be used to provide information on the physical characteristics of the AAA such as the AAA size and the presence of ILT. The results of the short investigation, discussed in Chapter 7, indicated that it was not possible to adequately characterise AAAs in term of size or ILT status using the data provided by the AAA detection program. This may have been because the program and the indices used in the program were optimised for AAA detection, not AAA characterisation. The results did however indicate that the reverse power density, reverse flow height and simultaneous forward and reverse flow under the systolic upstroke are features within the CFA waveform which may be useful for characterising AAA size. It may be possible to characterise the physical aspects of an AAA from the CFA waveform, using a different program optimised for the characterisation of AAA, possibly involving different analysis methods.

\section{$\underline{8.2 \text { Conclusions }}$}

The results of this research study suggest that automated feature analysis of the CFA Doppler waveform has potential as an alternative method to conventional B-mode ultrasound imaging for AAA screening. The overall response of the AAA detection program for the 46 patients with AAA and 41 patients without AAA and no significant atherosclerotic disease was a sensitivity of $100 \%$ for AAA detection with a specificity of $70.7 \%$. If these results are projected onto to the general population with no significant aorto-iliac or femoro-popliteal occlusive arterial disease, assuming the prevalence of lower limb PAD is around $12 \%$, this gives a sensitivity of around $100 \%$ and a specificity of around $70 \%$ for approximately $88 \%$ of the general population. 
Studies indicate that screening men over the age of 65 with B-mode ultrasound to detect AAA significantly reduces death rates and is cost-effective. However, although screening for AAA using conventional ultrasound techniques is cost effective, Bmode ultrasound scanning is hospital based, and requires a skilled operator to perform the investigation and analyse the images. CFA Doppler waveforms are measured by GPs and outpatient nurses using a simple hand held 'Pocket Doppler system'. Based on the findings of this research, it may be possible in future work to incorporate automated AAA detection software, based on CFA waveform feature analysis, into a hand-held Doppler system. This may provide an alternative screening tool for AAA which could offer a quick and more cost effective method for screening in GP surgeries and outpatient clinics.

If the performance of the AAA detection program is projected onto the potential screened population, assuming $93 \%$ of the screened population would not have significant atherosclerotic arterial disease, the AAA detection program would provide a sensitivity of around $100 \%$ and a specificity of around $70 \%$ for approximately $93 \%$ of the screened population. For the remaining $7 \%$ of the screened population with significant atherosclerotic arterial disease the sensitivity would be high (around $100 \%$ ) but the specificity would be low. From experience in the Doppler Ultrasound department at UHW approximately $25 \%$ of patients with significant lower limb arterial disease have significant proximal disease. For the $\sim 5 \%$ of screened patients with significant femoro-popliteal disease the specificity for AAA detection would be between $11 \%$ and $33 \%$. The inability of the disease detection program to function for the significant proximal disease groups may not be not critical to the function of the 
program as a screening tool because the proportion of the population with significant aorto-iliac atherosclerotic disease is very low.

If the AAA detection programs were to be implemented as an alternative method for screening for AAA in men over 50 years old, the original AAA detection program would provide an overall sensitivity of around $100 \%$ and a specificity of around $68 \%$ for AAA detection. It may be possible to combine the use of the 2 MATLAB programs with a simple technique such as an ABPI measurement to assess whether significant PAD is present. If the ABPI measurement was normal, the original program could be implemented to analyse the CFA waveforms. If the ABPI measurement indicated significant disease, the 'disease AAA detection program' could be implemented. This combined technique would provide around $100 \%$ sensitivity and a marginally improved specificity of around $70 \%$ for AAA detection in the screened population. Further work would be necessary to determine the feasibility of implementing the two AAA detection programs in conjunction with a simple method for arterial disease detection such as an ABPI measurement. The combined method may provide a slightly better specificity to AAA in the screened population than the original AAA detection program. However, from a practical view point, the implementation of the single AAA detection program would be much simpler and because such a small percentage $(\sim 7 \%)$ of the screened population would have significant atherosclerotic disease, this slight reduction in specificity may not be critical to the overall performance of the device in a screening program. The sensitivity and specificity levels provided by both the single and combined AAA detection programs are probably adequate for a simple tool to screen for AAA and 
indicate that objective analysis of CFA waveform features is a promising, alternative technique to conventional ultrasound for AAA detection.

It was not possible to adequately characterise AAAs in term of size or ILT status using the AAA detection program. This may be because the program and the indices used in the program were optimised for AAA detection, not AAA characterisation. The results from the investigation of the 6 individual indices show that the reverse power density, reverse flow height and simultaneous forward and reverse flow under the systolic upstroke are features within the AAA waveform which may be useful for characterising AAA size. It may be possible to characterise the physical aspects of an AAA from the CFA waveform using a different program optimised for the characterisation of AAA. In order to characterise AAA it is first necessary to be able to detect AAA, and most important result of the overall research project is that it is possible to detect AAA by analysis of the CFA waveform.

\subsection{Future Work}

Feature analysis of the CFA Doppler waveform may provide a promising, alternative method for AAA detection. However, if the technique were to be implemented as a possible screening tool for AAA, there are several considerations that need to be addressed. The technique would need to be tested on a much larger sample size with the relative proportions of normal and AAA patients with and without significant atherosclerotic disease more representative of the respective prevalences in the general population. It may then be possible to transfer the technique to simple handheld device, for example a hand held continuous wave Doppler 'pencil probe' system 
for CFA waveform measurement with integrated AAA detection software. The use of automated CFA waveform analysis in conjunction with a simple method to screen for PAD, for example ABPI, may also need to be investigated.

Further work is also necessary to provide explanations as to how the CFA waveform features that are present with an AAA arise. The modelling studies discussed in Chapter 1 have examined flows within aneurysms but further work is necessary, possibly with the application of fluid dynamics modelling and finite element analysis techniques to investigate the development of the flow disturbances and the subsequent effect on the blood flow in iliac and femoral arteries distal to the aneurysm.

Although it was not possible to characterise the physical characteristics of the AAAs in the patients used in this study using the AAA detection program. The modelling studies indicated that the flow disturbances set up within AAA models were dependent on the AAA size and shape. It may therefore be possible to characterise the physical aspects of an AAA from the CFA waveform using a program optimised for the characterisation of AAA, possibly involving different analysis methods. The modelling studies also indicated that turbulent flows set up within an AAA increase the arterial wall stress. Therefore, further analysis of the CFA waveform may also provide an aid for the prediction of rupture risk in future studies.

In the future it may be conceivable to detect AAA, determine the size and presence of ILT and predict the risk of rupture using a single, simple hand held Doppler device with integrated software. Potentially, application of this technique could significantly reduce costs to the NHS and save lives. 


\section{References}

Ashton HA, Buxton MJ, Day NE, et al. The Multicentre Aneurysm Screening Study (MASS) into the effect of abdominal aortic aneurysm screening on mortality in men: a randomised controlled trial. Lancet 2002;360(9345):1531-9.

Baur GM, Porter MJ, Eidemiller LR, Rosch J, Keller F. The Role of Angiography in Abdominal Aortic Aneurysm. The American Journal of Surgery $1978 ; 136$.

Cosgrove D, Meire H, Dewbury K. Foreword. In: B GB, ed. Abdominal and General Ultrasound. Edinburgh: Churchill Livingstone, 1993.

Curci JA, Baxter B, T., Thompson RW. Arterial Aneurysms: Etiologic Considerations. In: Rutherford RB, ed. Vascular Surgery. 6th ed. Denver: Elsevier Saunders, 2005.

Di Martino ES, Vorp DA. Effect of variation in intraluminal thrombus constitutive properties on abdominal aortic aneurysm wall stress. Ann Biomed Eng 2003;31(7):804-9.

Egelhoff CJ, Budwig RS, Elger DF, Khraishi TA, Johansen KH. Model studies of the flow in abdominal aortic aneurysms during resting and exercise conditions. Journal of Biomechanics 1999;32(12):1319-1329. 
Evans D, McDicken W, Skidmore R, Woodcock J, eds. Doppler Ultrasound: Physics, Instrumentation and Clincal Applications: John Wiley and Sons, 1989.

Fillinger MF, Raghavan ML, Marra SP, Cronenwett JL, Kennedy FE. In vivo analysis of mechanical wall stress and abdominal aortic aneurysm rupture risk. J Vasc Surg 2002;36(3):589-97.

Fish P. Physics and Instrumentation of Diagnostic Medical Ultrasound. Chichester: John Wiley and Sons Ltd, 1990.

Fowkes FG, Housley E, Cawood EH, Macintyre CC, Ruckley CV, Prescott RJ. Edinburgh Artery Study: prevalence of asymptomatic and symptomatic peripheral arterial disease in the general population. Int J Epidemiol 1991;20(2):384-92.

Greenhalgh RM, Powell JT. Screening men for aortic aneurysm. BMJ 2002;325(7373):1123-4.

Heather BP, Poskitt KR, Earnshaw JJ, Whyman M, Shaw E. Population screening reduces mortality rate from aortic aneurysm in men. Br J Surg 2000;87(6):750-3.

Hennerici D, Neuerburg-Heusler G. Chapter 7 Abdominal Arteries. Vascular Diagnosis with Ultrasound. New York: Thieme, 1998. 
Hennerici D, Neuerburg-Heusler G. Chapter 1 Ultrasound Procedures. Vascular Diagnosis with Ultrasound. New York: Thieme, 1998.

Hoskins P, Thrush A, K. M. Diagnostic ultrasound: physics and equipment. London: Greenwich Medical Media 2003.

Hua J, Mower WR. Simple geometric characteristics fail to reliably predict abdominal aortic aneurysm wall stresses. J Vasc Surg 2001;34(2):308-15.

Humphries KN, Hames TK, Chant ADB. Detection of arterial dilatation in the distal aorta-lower limb arteries using continuous wave Doppler ultrasound. Cardiovascular Research 1982;16:474-482.

Nichols W, O'Rourke, MF. McDonald's Blood Flow in Arteries. Theoretical, Experimental and Clinical Principles. London: Hodder Arnold, 2005.

Ostchega Y, Paulose-Ram R, Dillon CF, Gu Q, Hughes JP. Prevalence of peripheral arterial disease and risk factors in persons aged 60 and older: data from the National Health and Nutrition Examination Survey 19992004. J Am Geriatr Soc 2007;55(4):583-9.

Peattie RA, Asbury CL, Bluth EI, Ruberti JW. Steady flow in models of abdominal aortic aneurysms. Part I: Investigation of the velocity patterns. J Ultrasound Med 1996;15(10):679-88. 
Peattie RA, Schrader T, Bluth EI, Comstock CE. Development of turbulence in steady flow through models of abdominal aortic aneurysms. J Ultrasound Med 1994;13(6):467-72.

Pretre R. Facts, at last, on management of small infrarenal aortic aneurysms. Lancet 1998;352(9141).

Schrader T, Peattie RA, Bluth EI, Comstock CE. A Qualitative Investigation of Turbulence in Flow through a Model Abdominal Aortic-Aneurysm. Investigative Radiology 1992;27(7):515-519.

Schurink GW, van Baalen JM, Visser MJ, van Bockel JH. Thrombus within an aortic aneurysm does not reduce pressure on the aneurysmal wall. J Vasc Surg 2000;31(3):501-6.

Scott RA, Bridgewater SG, Ashton HA. Randomized clinical trial of screening for abdominal aortic aneurysm in women. Br J Surg 2002;89(3):283-5.

Scott RA, Wilson NM, Ashton HA, Kay DN. Influence of screening on the incidence of ruptured abdominal aortic aneurysm: 5-year results of a randomized controlled study. Br J Surg 1995;82(8):1066-70.

Seeley RR, Stephens TD, Tate P. Anatomy and Physiology. 5th ed. Boston: McGraw Hill, 2000. 
Semmens JB, Norman PE, Lawrence-Brown MM, Holman CD. Influence of gender on outcome from ruptured abdominal aortic aneurysm. Br J Surg $2000 ; 87(2): 191-4$

Smith R. Britain needs to screen for aortic aneurysms. BMJ 1994(308):358-359.

Solomon EP, Schmidt RR, Adragna PJ. Circulation: The Blood Vessels. Human Anatomy and Physiology. International 2nd Edition ed. Ft. Worth: Saunders College Publishing, 1990.

Steinman DA, Vorp DA, Ethier CR. Computational modeling of arterial biomechanics: insights into pathogenesis and treatment of vascular disease. J Vasc Surg 2003;37(5):1118-28.

Sumner DS, Zierler RE. Vascular Physiology: Essential Haemodynamic Principles. In: Rutherford RB, ed. Vascular Surgery. 6th ed. Denver: Elsevier Saunders, 2005.

Vorp D, Raghavan B, Marshall W, Webster M. Mechanical wall stress in abdominal aortic aneurysm: Influence of diameter and asymmetry. Journal of Vascular Surgery 1998;27(4):632-639.

Wilmink AB, Quick CR, Hubbard CS, Day NE. Effectiveness and cost of screening for abdominal aortic aneurysm: results of a population screening program. J Vasc Surg 2003;38(1):72-7. 
Yehuda G. Wolf Bonnie L. Jhonson, , Bradlry B. Hill, Geoffrey D. Rubin, Thomas J. Fogarty and Christopher K. Zarind. Duplex Scannoing Versus CT Angiography for Preoperative Evaluation of Endovascular Abdominal Aortic Aneurysm Repair. Journal of aortic aneurysm models. Fluid Dynamics Research 2001(29):81-113.Vascular Surgery 2000;32(6).

Yip T, Yu S. Cyclic transition to turbulence in rigid abdominal aortic aneurysm models. Fluid Dynamics Research 2001(29):81-113.

Yip T, Yu S. Oscillatory flows in straight tubes with an axisymmetric bulge. Experimental Thermal and Fluid Science 2002(26):947-961.

Yip TH, Yu SCM. Cyclic flow characteristics in an idealized asymmetric abdominal aortic aneurysm model. Proceedings of the Institution of Mechanical Engineers Part H- Journal of Engineering in Medicine 2003;217(H1):27-39.

Yu S. Steady and pulsatile flows in Abdominal Aortic Aneurysm Models usign image particle velocimetry. International Journal of Heat and Fluid Flow $2000 ; 21: 74-83$.

Zarins CK, Xu CXU, Glagov S. Artery Wall Pathology in Atherosclerosis. In: Rutherford RB, ed. Vascular Surgery. 6th ed. Denver: Elsevier Saunders, 2005 . 
Appendix 1: Patient Data Summary for AAA Patient Groups with No Significant

$\underline{\text { Iliac or Femoro-Popliteal Disease }}$

Table A1.1: Patient Data Summary for AAA with No Significant Atherosclerotic Disease Study Group

\begin{tabular}{|c|c|c|c|c|c|c|c|}
\hline $\begin{array}{l}\text { Patient } \\
\text { Number }\end{array}$ & $\begin{array}{c}\text { Age } \\
\text { (Years) }\end{array}$ & $\begin{array}{l}\text { Maximum } \\
\text { Abdominal } \\
\text { Aorta } \\
\text { Diameter } \\
(\mathbf{c m})\end{array}$ & ILT & $\begin{array}{c}\text { Residual } \\
\text { Lumen } \\
\text { (cm) }\end{array}$ & Leg & $\begin{array}{c}\text { Iliac Artery } \\
\text { Disease } \\
\text { State }\end{array}$ & $\begin{array}{c}\text { Femoro- } \\
\text { Popliteal } \\
\text { Disease State }\end{array}$ \\
\hline \multirow{2}{*}{1} & \multirow{2}{*}{81} & \multirow{2}{*}{5.0} & \multirow{2}{*}{ Yes } & \multirow{2}{*}{2.5} & Left & $\begin{array}{c}\text { Very } \\
\text { tortuous } \\
\text { iliac arteries } \\
\text { but no } \\
\text { significant } \\
\text { disease } \\
\end{array}$ & $\begin{array}{l}\text { Minor } \\
\text { calcification }\end{array}$ \\
\hline & & & & & Right & $\begin{array}{c}\text { Very } \\
\text { tortuous } \\
\text { iliac arteries } \\
\text { but no } \\
\text { significant } \\
\text { disease } \\
\end{array}$ & $\begin{array}{c}\text { Minor } \\
\text { calcification }\end{array}$ \\
\hline \multirow{2}{*}{2} & \multirow{2}{*}{68} & \multirow{2}{*}{3.8} & \multirow{2}{*}{ No } & \multirow{2}{*}{ - } & Left & $\begin{array}{c}\text { Minor } \\
\text { calcification }\end{array}$ & $\begin{array}{c}\text { Minor } \\
\text { calcification }\end{array}$ \\
\hline & & & & & Right & $\begin{array}{c}\text { Minor } \\
\text { calcification }\end{array}$ & $\begin{array}{c}\text { Minor } \\
\text { calcification }\end{array}$ \\
\hline \multirow{2}{*}{3} & \multirow{2}{*}{71} & \multirow{2}{*}{5.0} & \multirow{2}{*}{ Yes } & \multirow{2}{*}{2.8} & Left & No disease & No disease \\
\hline & & & & & Right & No disease & No disease \\
\hline \multirow{2}{*}{4} & \multirow{2}{*}{77} & \multirow{2}{*}{4.1} & \multirow{2}{*}{ Yes } & \multirow{2}{*}{2.2} & Left & No disease & No disease \\
\hline & & & & & Right & No disease & No disease \\
\hline \multirow{2}{*}{5} & \multirow{2}{*}{66} & \multirow{2}{*}{4.0} & \multirow{2}{*}{ Yes } & \multirow{2}{*}{3.2} & Left & No disease & No disease \\
\hline & & & & & Right & No disease & No disease \\
\hline \multirow{2}{*}{6} & \multirow{2}{*}{76} & \multirow{2}{*}{6.4} & \multirow{2}{*}{ Yes } & \multirow{2}{*}{3.9} & Left & $\begin{array}{c}\text { Tortuous } \\
\text { iliac arteries } \\
\text { but no } \\
\text { significant } \\
\text { disease }\end{array}$ & $\begin{array}{c}\text { Minor } \\
\text { calcification }\end{array}$ \\
\hline & & & & & Right & $\begin{array}{c}\text { Tortuous } \\
\text { iliac arteries } \\
\text { but no } \\
\text { significant } \\
\text { disease } \\
\end{array}$ & $\begin{array}{c}\text { Minor } \\
\text { calcification }\end{array}$ \\
\hline \multirow{2}{*}{7} & \multirow{2}{*}{65} & \multirow{2}{*}{3.0} & \multirow{2}{*}{ No } & \multirow{2}{*}{-} & Left & No disease & No disease \\
\hline & & & & & Right & No disease & No disease \\
\hline \multirow{2}{*}{8} & \multirow{2}{*}{54} & 58 & $\mathrm{No}$ & 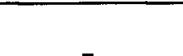 & Left & No disease & No disease \\
\hline & & 5.8 & NO & - & Right & No disease & No disease \\
\hline
\end{tabular}


Table A1.1 continued:

\begin{tabular}{|c|c|c|c|c|c|c|c|}
\hline $\begin{array}{l}\text { Patient } \\
\text { Number }\end{array}$ & $\begin{array}{c}\text { Age } \\
\text { (Years) }\end{array}$ & $\begin{array}{l}\text { Maximum } \\
\text { Abdominal } \\
\text { Aorta } \\
\text { Diameter } \\
\text { (cm) }\end{array}$ & ILT & $\begin{array}{l}\text { Residual } \\
\text { Lumen } \\
\text { (cm) }\end{array}$ & Leg & $\begin{array}{c}\text { Iliac Artery } \\
\text { Disease } \\
\text { State }\end{array}$ & $\begin{array}{c}\text { Femoro- } \\
\text { Popliteal } \\
\text { Disease State }\end{array}$ \\
\hline \multirow{2}{*}{9} & \multirow{2}{*}{68} & \multirow{2}{*}{4.1} & \multirow{2}{*}{ No } & \multirow{2}{*}{-} & Left & No disease & No disease \\
\hline & & & & & Right & No disease & No disease \\
\hline \multirow{2}{*}{10} & \multirow{2}{*}{85} & \multirow{2}{*}{4.3} & \multirow{2}{*}{ No } & \multirow{2}{*}{-} & Left & $\begin{array}{c}\text { Tortuous } \\
\text { iliac arteries } \\
\text { but no } \\
\text { significant } \\
\text { disease } \\
\end{array}$ & $\begin{array}{c}\text { Minor } \\
\text { calcification }\end{array}$ \\
\hline & & & & & Right & $\begin{array}{c}\text { Tortuous } \\
\text { iliac arteries } \\
\text { but no } \\
\text { significant } \\
\text { disease } \\
\end{array}$ & $\begin{array}{c}\text { Minor } \\
\text { calcification }\end{array}$ \\
\hline \multirow{2}{*}{11} & \multirow{2}{*}{71} & \multirow{2}{*}{5.0} & \multirow{2}{*}{ No } & \multirow{2}{*}{ - } & Left & No disease & No disease \\
\hline & & & & & Right & No disease & No disease \\
\hline \multirow{2}{*}{12} & \multirow{2}{*}{81} & \multirow{2}{*}{4.0} & \multirow{2}{*}{ No } & \multirow{2}{*}{ - } & Left & $\begin{array}{c}\text { Minor } \\
\text { calcification }\end{array}$ & $\begin{array}{c}\text { Minor } \\
\text { calcification }\end{array}$ \\
\hline & & & & & Right & $\begin{array}{c}\text { Minor } \\
\text { calcification }\end{array}$ & $\begin{array}{c}\text { Minor } \\
\text { calcification }\end{array}$ \\
\hline \multirow{2}{*}{13} & \multirow{2}{*}{55} & \multirow{2}{*}{4.5} & \multirow{2}{*}{ Yes } & \multirow{2}{*}{3.0} & Left & $\begin{array}{c}\text { Minor } \\
\text { calcification }\end{array}$ & $\begin{array}{c}\text { Minor } \\
\text { calcification }\end{array}$ \\
\hline & & & & & Right & $\begin{array}{c}\text { Minor } \\
\text { calcification }\end{array}$ & $\begin{array}{c}\text { Minor } \\
\text { calcification }\end{array}$ \\
\hline \multirow{2}{*}{14} & \multirow{2}{*}{74} & \multirow{2}{*}{5.0} & \multirow{2}{*}{ No } & \multirow{2}{*}{-} & Left & $\begin{array}{c}\text { Minor } \\
\text { calcification } \\
\end{array}$ & $\begin{array}{c}\text { Minor } \\
\text { calcification } \\
\end{array}$ \\
\hline & & & & & Right & $\begin{array}{c}\text { Minor } \\
\text { calcification }\end{array}$ & $\begin{array}{c}\text { Minor } \\
\text { calcification }\end{array}$ \\
\hline \multirow{2}{*}{15} & \multirow{2}{*}{71} & \multirow{2}{*}{4.1} & \multirow{2}{*}{ Yes } & & Left & $\begin{array}{c}\text { Minor } \\
\text { calcification }\end{array}$ & $\begin{array}{c}\text { Minor } \\
\text { calcification }\end{array}$ \\
\hline & & & & 2.7 & Right & $\begin{array}{c}\text { Minor } \\
\text { calcification }\end{array}$ & $\begin{array}{c}\text { Minor } \\
\text { calcification }\end{array}$ \\
\hline 16 & 65 & 4.6 & Yes & 3.6 & Left & No disease & No disease \\
\hline & נo & 4.0 & & 3.0 & Right & No disease & No disease \\
\hline & & & & & Left & No disease & $\begin{array}{c}\text { Minor } \\
\text { calcification }\end{array}$ \\
\hline 17 & 85 & 4.0 & No & - & Right & No disease & $\begin{array}{c}\text { Minor } \\
\text { calcification }\end{array}$ \\
\hline & 55 & 35 & $\mathrm{No}_{0}$ & - & Left & $\begin{array}{c}\text { Minor } \\
\text { calcification }\end{array}$ & $\begin{array}{c}\text { Minor } \\
\text { calcification }\end{array}$ \\
\hline 18 & 5 & 3.5 & No & - & Right & $\begin{array}{c}\text { Minor } \\
\text { calcification }\end{array}$ & $\begin{array}{c}\text { Minor } \\
\text { calcification }\end{array}$ \\
\hline & & & & & Left & $\begin{array}{c}\text { Minor } \\
\text { calcification }\end{array}$ & $\begin{array}{c}\text { Minor } \\
\text { calcification }\end{array}$ \\
\hline 19 & 10 & 4.9 & No & - & Right & $\begin{array}{c}\text { Minor } \\
\text { calcification }\end{array}$ & $\begin{array}{c}\text { Minor } \\
\text { calcification }\end{array}$ \\
\hline
\end{tabular}


Table A1.1 continued:

\begin{tabular}{|c|c|c|c|c|c|c|c|}
\hline $\begin{array}{l}\text { Patient } \\
\text { Number }\end{array}$ & $\begin{array}{c}\text { Age } \\
\text { (Years) }\end{array}$ & $\begin{array}{l}\text { Maximum } \\
\text { Abdominal } \\
\text { Aorta } \\
\text { Diameter } \\
\text { (cm) } \\
\end{array}$ & ILT & $\begin{array}{c}\text { Residual } \\
\text { Lumen } \\
(\mathbf{c m})\end{array}$ & Leg & $\begin{array}{c}\text { Iliac Artery } \\
\text { Disease } \\
\text { State }\end{array}$ & $\begin{array}{c}\text { Femoro- } \\
\text { Popliteal } \\
\text { Disease State }\end{array}$ \\
\hline \multirow{2}{*}{20} & \multirow{2}{*}{77} & \multirow{2}{*}{4.4} & \multirow{2}{*}{ Yes } & \multirow{2}{*}{2.5} & Left & $\begin{array}{c}\text { Minor } \\
\text { calcification }\end{array}$ & $\begin{array}{c}\text { Minor } \\
\text { calcification }\end{array}$ \\
\hline & & & & & Right & $\begin{array}{c}\text { Minor } \\
\text { calcification }\end{array}$ & $\begin{array}{c}\text { Minor } \\
\text { calcification }\end{array}$ \\
\hline \multirow{2}{*}{21} & \multirow{2}{*}{40} & \multirow{2}{*}{5.2} & \multirow{2}{*}{ No } & \multirow{2}{*}{-} & Left & $\begin{array}{c}\text { Minor } \\
\text { calcification }\end{array}$ & $\begin{array}{c}\text { Minor } \\
\text { calcification }\end{array}$ \\
\hline & & & & & Right & $\begin{array}{c}\text { Minor } \\
\text { calcification }\end{array}$ & $\begin{array}{c}\text { Minor } \\
\text { calcification }\end{array}$ \\
\hline \multirow{2}{*}{22} & \multirow{2}{*}{68} & \multirow{2}{*}{5.8} & \multirow{2}{*}{ Yes } & \multirow{2}{*}{2.0} & Left & $\begin{array}{c}\text { Minor } \\
\text { calcification }\end{array}$ & $\begin{array}{c}\text { Minor } \\
\text { calcification }\end{array}$ \\
\hline & & & & & Right & $\begin{array}{c}\text { Minor } \\
\text { calcification }\end{array}$ & $\begin{array}{c}\text { Minor } \\
\text { calcification }\end{array}$ \\
\hline \multirow{2}{*}{23} & \multirow{2}{*}{69} & \multirow{2}{*}{5.4} & \multirow{2}{*}{ Yes } & \multirow{2}{*}{2.3} & Left & $\begin{array}{c}\text { Minor } \\
\text { calcification }\end{array}$ & $\begin{array}{c}\text { Minor } \\
\text { calcification }\end{array}$ \\
\hline & & & & & Right & $\begin{array}{c}\text { Minor } \\
\text { calcification }\end{array}$ & $\begin{array}{c}\text { Minor } \\
\text { calcification }\end{array}$ \\
\hline \multirow{2}{*}{24} & \multirow{2}{*}{83} & \multirow{2}{*}{6.0} & \multirow{2}{*}{ Yes } & \multirow{2}{*}{2.0} & Left & $\begin{array}{c}\text { Minor } \\
\text { calcification }\end{array}$ & $\begin{array}{c}\text { Minor } \\
\text { calcification }\end{array}$ \\
\hline & & & & & Right & $\begin{array}{c}\text { Minor } \\
\text { calcification }\end{array}$ & $\begin{array}{c}\text { Minor } \\
\text { calcification }\end{array}$ \\
\hline \multirow{2}{*}{25} & \multirow{2}{*}{85} & \multirow{2}{*}{4.5} & \multirow{2}{*}{ No } & \multirow{2}{*}{-} & Left & $\begin{array}{c}\text { Minor } \\
\text { calcification }\end{array}$ & $\begin{array}{c}\text { Minor } \\
\text { calcification }\end{array}$ \\
\hline & & & & & Right & $\begin{array}{c}\text { Minor } \\
\text { calcification }\end{array}$ & $\begin{array}{c}\text { Minor } \\
\text { calcification }\end{array}$ \\
\hline \multirow[t]{2}{*}{26} & \multirow[t]{2}{*}{67} & \multirow[t]{2}{*}{3.2} & \multirow[t]{2}{*}{ No } & \multirow[t]{2}{*}{-} & Left & $\begin{array}{c}\text { Moderate } \\
\text { calcification }\end{array}$ & $\begin{array}{c}\text { Moderate } \\
\text { calcification } \\
\text { but no } \\
\text { significant } \\
\text { focal lesions }\end{array}$ \\
\hline & & & & & Right & $\begin{array}{c}\text { Minor } \\
\text { calcification }\end{array}$ & $\begin{array}{c}\text { Minor } \\
\text { calcification }\end{array}$ \\
\hline \multirow{2}{*}{27} & \multirow{2}{*}{69} & 38 & Ye & 21 & Left & $\begin{array}{l}\text { Moderate } \\
\text { calcification }\end{array}$ & $\begin{array}{c}\text { Moderate } \\
\text { calcification } \\
\text { but no } \\
\text { significant } \\
\text { focal lesions }\end{array}$ \\
\hline & & 3.8 & Yes & 2.1 & Right & $\begin{array}{l}\text { Moderate } \\
\text { calcification }\end{array}$ & $\begin{array}{c}\text { Moderate } \\
\text { calcification } \\
\text { but no } \\
\text { significant } \\
\text { focal lesions }\end{array}$ \\
\hline
\end{tabular}


Table A1.1 continued:

\begin{tabular}{|c|c|c|c|c|c|c|c|}
\hline $\begin{array}{l}\text { Patient } \\
\text { Number }\end{array}$ & $\begin{array}{c}\text { Age } \\
\text { (Years) }\end{array}$ & $\begin{array}{l}\text { Maximum } \\
\text { Abdominal } \\
\text { Aorta } \\
\text { Diameter } \\
\text { (cm) }\end{array}$ & ILT & $\begin{array}{l}\text { Residual } \\
\text { Lumen } \\
\text { (cm) }\end{array}$ & Leg & $\begin{array}{c}\text { Iliac Artery } \\
\text { Disease } \\
\text { State }\end{array}$ & $\begin{array}{c}\text { Femoro- } \\
\text { Popliteal } \\
\text { Disease State }\end{array}$ \\
\hline \multirow{2}{*}{28} & \multirow{2}{*}{75} & \multirow{2}{*}{4.5} & \multirow{2}{*}{ No } & \multirow{2}{*}{-} & Left & $\begin{array}{c}\text { Minor } \\
\text { calcification }\end{array}$ & $\begin{array}{c}\text { Minor } \\
\text { calcification }\end{array}$ \\
\hline & & & & & Right & $\begin{array}{c}\text { Minor } \\
\text { calcification }\end{array}$ & $\begin{array}{c}\text { Minor } \\
\text { calcification }\end{array}$ \\
\hline \multirow{2}{*}{29} & \multirow{2}{*}{85} & \multirow{2}{*}{5.7} & \multirow{2}{*}{ Yes } & \multirow{2}{*}{2.4} & Left & $\begin{array}{c}\text { Minor } \\
\text { calcification }\end{array}$ & $\begin{array}{c}\text { Minor } \\
\text { calcification }\end{array}$ \\
\hline & & & & & Right & $\begin{array}{c}\text { Minor } \\
\text { calcification }\end{array}$ & $\begin{array}{c}\text { Minor } \\
\text { calcification }\end{array}$ \\
\hline \multirow{2}{*}{30} & \multirow{2}{*}{69} & \multirow{2}{*}{3.5} & \multirow{2}{*}{ No } & \multirow{2}{*}{-} & Left & $\begin{array}{c}\text { Minor } \\
\text { calcification }\end{array}$ & $\begin{array}{c}\text { Minor } \\
\text { calcification }\end{array}$ \\
\hline & & & & & Right & $\begin{array}{c}\text { Minor } \\
\text { calcification }\end{array}$ & $\begin{array}{c}\text { Minor } \\
\text { calcification }\end{array}$ \\
\hline
\end{tabular}


Table A1.2: Patient Data Summary for AAA with No Significant Atherosclerotic Disease Test Group

\begin{tabular}{|c|c|c|c|c|c|c|c|}
\hline $\begin{array}{l}\text { Patient } \\
\text { Number }\end{array}$ & $\begin{array}{c}\text { Age } \\
\text { (Years) }\end{array}$ & $\begin{array}{c}\text { Maximum } \\
\text { Abdominal } \\
\text { Aorta } \\
\text { Diameter (cm) }\end{array}$ & ILT & $\begin{array}{c}\text { Residual } \\
\text { Lumen } \\
\text { (cm) }\end{array}$ & Leg & $\begin{array}{c}\text { Iliac Artery } \\
\text { Disease } \\
\text { State }\end{array}$ & $\begin{array}{c}\text { Femoro- } \\
\text { Popliteal } \\
\text { Disease State }\end{array}$ \\
\hline \multirow{2}{*}{1} & \multirow{2}{*}{61} & \multirow{2}{*}{3.1} & \multirow{2}{*}{ No } & \multirow{2}{*}{-} & Left & No disease & No disease \\
\hline & & & & & Right & No disease & No disease \\
\hline \multirow[b]{2}{*}{2} & \multirow[b]{2}{*}{83} & \multirow[b]{2}{*}{4.6} & \multirow[b]{2}{*}{ No } & \multirow[b]{2}{*}{-} & Left & $\begin{array}{c}\text { Minor } \\
\text { calcification }\end{array}$ & $\begin{array}{c}\text { Minor } \\
\text { calcification }\end{array}$ \\
\hline & & & & & Right & $\begin{array}{c}\text { Minor } \\
\text { calcification }\end{array}$ & $\begin{array}{c}\text { Moderate } \\
\text { calcification } \\
\text { but no } \\
\text { significant } \\
\text { focal lesions }\end{array}$ \\
\hline \multirow{2}{*}{3} & \multirow{2}{*}{73} & \multirow{2}{*}{3.5} & \multirow{2}{*}{ No } & \multirow{2}{*}{-} & Left & No disease & No disease \\
\hline & & & & & Right & No disease & No disease \\
\hline \multirow{2}{*}{4} & \multirow{2}{*}{77} & \multirow{2}{*}{3.5} & \multirow{2}{*}{ No } & \multirow{2}{*}{ - } & Left & $\begin{array}{c}\text { Minor } \\
\text { calcification }\end{array}$ & $\begin{array}{c}\text { Minor } \\
\text { calcification }\end{array}$ \\
\hline & & & & & Right & $\begin{array}{c}\text { Minor } \\
\text { calcification }\end{array}$ & $\begin{array}{c}\text { Minor } \\
\text { calcification }\end{array}$ \\
\hline \multirow[t]{2}{*}{5} & \multirow[t]{2}{*}{83} & \multirow[t]{2}{*}{5.2} & \multirow[t]{2}{*}{ No } & \multirow[t]{2}{*}{-} & Left & $\begin{array}{c}\text { Tortuous } \\
\text { EIA. } \\
\text { Moderate } \\
\text { calcification }\end{array}$ & $\begin{array}{c}\text { Moderate } \\
\text { calcification } \\
\text { but no } \\
\text { significant } \\
\text { focal lesions }\end{array}$ \\
\hline & & & & & Right & $\begin{array}{c}\text { Minor } \\
\text { calcification }\end{array}$ & $\begin{array}{c}\text { Minor } \\
\text { calcification }\end{array}$ \\
\hline \multirow[t]{2}{*}{6} & \multirow[t]{2}{*}{78} & \multirow[t]{2}{*}{4.0} & \multirow[t]{2}{*}{ No } & \multirow[t]{2}{*}{-} & Left & $\begin{array}{c}\text { Minor } \\
\text { calcification }\end{array}$ & $\begin{array}{c}\text { Minor } \\
\text { calcification } \\
\text { but no } \\
\text { significant } \\
\text { disease } \\
\end{array}$ \\
\hline & & & & & Right & $\begin{array}{c}\text { Minor } \\
\text { calcification }\end{array}$ & $\begin{array}{c}\text { Minor } \\
\text { calcification }\end{array}$ \\
\hline \multirow{2}{*}{7} & \multirow{2}{*}{78} & \multirow{2}{*}{3.5} & \multirow{2}{*}{ Yes } & \multirow{2}{*}{2.3} & Left & No disease & No disease \\
\hline & & & & & Right & No disease & No disease \\
\hline
\end{tabular}


Table A1.3: Patient Data Summary for AAA with No Significant Atherosclerotic

Disease with Additional Aneurysmal Disease Group

\begin{tabular}{|c|c|c|c|c|c|c|c|c|}
\hline $\begin{array}{l}\text { Patient } \\
\text { Number }\end{array}$ & $\begin{array}{c}\text { Age } \\
\text { (Years) }\end{array}$ & $\begin{array}{l}\text { Maximum } \\
\text { Abdominal } \\
\text { Aorta } \\
\text { Diameter } \\
\text { (cm) } \\
\end{array}$ & ILT & $\begin{array}{c}\text { Residual } \\
\text { Lumen } \\
\text { (cm) }\end{array}$ & Leg & $\begin{array}{c}\text { Iliac Artery } \\
\text { Disease } \\
\text { State }\end{array}$ & $\begin{array}{c}\text { Femoro- } \\
\text { Popliteal } \\
\text { Disease } \\
\text { State }\end{array}$ & $\begin{array}{c}\text { Additional } \\
\text { Aneurysmal } \\
\text { Disease }\end{array}$ \\
\hline \multirow{2}{*}{1} & \multirow{2}{*}{68} & \multirow{2}{*}{5.7} & \multirow{2}{*}{ Yes } & \multirow{2}{*}{3.6} & Left & No disease & No disease & $\begin{array}{c}1.9 \mathrm{~cm} \\
\text { popliteal } \\
\text { aneurysm }\end{array}$ \\
\hline & & & & & Right & No disease & No disease & $\begin{array}{c}1.6 \mathrm{~cm} \\
\text { popliteal } \\
\text { aneurysm } \\
\end{array}$ \\
\hline \multirow{2}{*}{2} & \multirow{2}{*}{69} & \multirow{2}{*}{3.7} & \multirow{2}{*}{ Yes } & \multirow{2}{*}{3.3} & Left & No disease & No disease & $\begin{array}{l}3.3 \mathrm{~cm} \text { CIA } \\
\text { aneurysm }\end{array}$ \\
\hline & & & & & Right & No disease & No disease & $\begin{array}{l}2.2 \mathrm{~cm} \mathrm{CIA} \\
\text { aneurysm }\end{array}$ \\
\hline \multirow{2}{*}{3} & \multirow{2}{*}{76} & \multirow{2}{*}{3.7} & \multirow{2}{*}{ No } & \multirow{2}{*}{ - } & Left & $\begin{array}{c}\text { Minor } \\
\text { calcification }\end{array}$ & $\begin{array}{c}\text { Minor } \\
\text { calcification }\end{array}$ & 2. \\
\hline & & & & & Right & $\begin{array}{c}\text { Minor } \\
\text { calcification }\end{array}$ & $\begin{array}{c}\text { Minor } \\
\text { calcification }\end{array}$ & $\begin{array}{l}1.7 \mathrm{~cm} \mathrm{CIA} \\
\text { aneurysm }\end{array}$ \\
\hline \multirow{2}{*}{4} & \multirow{2}{*}{76} & \multirow{2}{*}{5.1} & \multirow{2}{*}{ Yes } & \multirow{2}{*}{3.1} & Left & No disease & No disease & $\begin{array}{l}2.1 \mathrm{~cm} \text { CIA } \\
\text { aneurysm }\end{array}$ \\
\hline & & & & & Right & No disease & No disease & $\begin{array}{l}2.5 \mathrm{~cm} \text { CIA } \\
\text { aneurysm }\end{array}$ \\
\hline \multirow{2}{*}{5} & \multirow{2}{*}{77} & \multirow{2}{*}{4.7} & \multirow{2}{*}{ Yes } & \multirow{2}{*}{3.1} & Left & $\begin{array}{c}\text { Minor } \\
\text { calcification }\end{array}$ & $\begin{array}{c}\text { Minor } \\
\text { calcification }\end{array}$ & $\begin{array}{l}1.6 \mathrm{~cm} \mathrm{CIA} \\
\text { aneurysm }\end{array}$ \\
\hline & & & & & Right & $\begin{array}{c}\text { Minor } \\
\text { calcification }\end{array}$ & $\begin{array}{c}\text { Minor } \\
\text { calcification }\end{array}$ & $\begin{array}{c}1.9 \mathrm{~cm} \text { CIA } \\
\text { aneurysm }\end{array}$ \\
\hline \multirow{2}{*}{6} & \multirow{2}{*}{75} & \multirow{2}{*}{3.5} & \multirow{2}{*}{ No } & \multirow{2}{*}{ - } & Left & $\begin{array}{c}\text { Minor } \\
\text { calcification }\end{array}$ & $\begin{array}{c}\text { Minor } \\
\text { calcification }\end{array}$ & $\begin{array}{c}3.0 \mathrm{~cm} \\
\text { popliteal } \\
\text { aneurysm }\end{array}$ \\
\hline & & & & & Right & $\begin{array}{c}\text { Minor } \\
\text { calcification }\end{array}$ & $\begin{array}{c}\text { Minor } \\
\text { calcification }\end{array}$ & $\begin{array}{c}1.9 \mathrm{~cm} \\
\text { popliteal } \\
\text { aneurysm }\end{array}$ \\
\hline \multirow{2}{*}{7} & \multirow{2}{*}{88} & \multirow{2}{*}{5.2} & \multirow{2}{*}{ Yes } & \multirow{2}{*}{2.0} & Left & $\begin{array}{c}\text { Minor } \\
\text { calcification }\end{array}$ & $\begin{array}{c}\text { Minor } \\
\text { calcification }\end{array}$ & $\begin{array}{c}2.0 \mathrm{~cm} \text { CIA } \\
\text { aneurysm }\end{array}$ \\
\hline & & & & & Right & $\begin{array}{c}\text { Minor } \\
\text { calcification }\end{array}$ & $\begin{array}{c}\text { Minor } \\
\text { calcification }\end{array}$ & $\begin{array}{l}1.5 \mathrm{~cm} \text { CIA } \\
\text { aneurysm }\end{array}$ \\
\hline 0 & 71 & 38 & No & & Left & $\begin{array}{c}\text { Minor } \\
\text { calcification }\end{array}$ & $\begin{array}{c}\text { Minor } \\
\text { calcification }\end{array}$ & $\begin{array}{l}1.8 \mathrm{~cm} \mathrm{CIA} \\
\text { aneurysm }\end{array}$ \\
\hline 8 & 14 & 5.0 & NO & - & Right & $\begin{array}{c}\text { Minor } \\
\text { calcification }\end{array}$ & $\begin{array}{c}\text { Minor } \\
\text { calcification }\end{array}$ & $\begin{array}{l}1.6 \mathrm{~cm} \mathrm{CIA} \\
\text { aneurysm }\end{array}$ \\
\hline 0 & 78 & 30 & No & & Left & No disease & No disease & $\begin{array}{l}1.5 \mathrm{~cm} \text { CIA } \\
\text { aneurysm }\end{array}$ \\
\hline 9 & 18 & 3.0 & No & - & Right & No disease & No disease & $\begin{array}{l}1.5 \mathrm{~cm} \text { CIA } \\
\text { aneurysm }\end{array}$ \\
\hline
\end{tabular}


Appendix 2: Patient Data Summary for Normal Patient Groups

Table A2.1: Patient Data Summary for Normal Study Group

\begin{tabular}{|c|c|c|c|c|c|}
\hline $\begin{array}{l}\text { Patient } \\
\text { Number }\end{array}$ & $\begin{array}{c}\text { Age } \\
\text { (Years) }\end{array}$ & $\begin{array}{c}\text { Maximum } \\
\text { Abdominal } \\
\text { Aorta Diameter } \\
(\mathbf{c m}) \\
\end{array}$ & Leg & $\begin{array}{l}\text { Iliac Artery } \\
\text { Disease State }\end{array}$ & $\begin{array}{c}\text { Femoro-Popliteal } \\
\text { Disease State }\end{array}$ \\
\hline \multirow{2}{*}{1} & \multirow{2}{*}{60} & \multirow{2}{*}{1.8} & Left & No disease & No disease \\
\hline & & & Right & No disease & No disease \\
\hline \multirow{2}{*}{2} & \multirow{2}{*}{60} & \multirow{2}{*}{1.5} & Left & No disease & No disease \\
\hline & & & Right & No disease & No disease \\
\hline \multirow{2}{*}{3} & \multirow{2}{*}{52} & \multirow{2}{*}{1.9} & Left & No disease & No disease \\
\hline & & & Right & No disease & No disease \\
\hline \multirow{2}{*}{4} & \multirow{2}{*}{55} & \multirow{2}{*}{1.5} & Left & No disease & No disease \\
\hline & & & Right & No disease & No disease \\
\hline \multirow{2}{*}{5} & \multirow{2}{*}{72} & \multirow{2}{*}{2.0} & Left & $\begin{array}{c}\text { Minor } \\
\text { calcification }\end{array}$ & $\begin{array}{c}\text { Minor } \\
\text { calcification }\end{array}$ \\
\hline & & & Right & $\begin{array}{c}\text { Minor } \\
\text { calcification }\end{array}$ & $\begin{array}{c}\text { Minor } \\
\text { calcification }\end{array}$ \\
\hline \multirow{2}{*}{6} & \multirow{2}{*}{72} & \multirow{2}{*}{1.3} & Left & $\begin{array}{c}\text { Minor } \\
\text { calcification }\end{array}$ & $\begin{array}{c}\text { Minor } \\
\text { calcification }\end{array}$ \\
\hline & & & Right & $\begin{array}{c}\text { Minor } \\
\text { calcification }\end{array}$ & $\begin{array}{c}\text { Minor } \\
\text { calcification }\end{array}$ \\
\hline \multirow{2}{*}{7} & \multirow{2}{*}{51} & \multirow{2}{*}{1.4} & Left & No disease & No disease \\
\hline & & & Right & No disease & No disease \\
\hline \multirow{2}{*}{8} & \multirow{2}{*}{63} & \multirow{2}{*}{1.2} & Left & $\begin{array}{c}\text { Minor } \\
\text { calcification }\end{array}$ & $\begin{array}{c}\text { Minor } \\
\text { calcification }\end{array}$ \\
\hline & & & Right & $\begin{array}{c}\text { Minor } \\
\text { calcification }\end{array}$ & $\begin{array}{c}\text { Minor } \\
\text { calcification }\end{array}$ \\
\hline \multirow[t]{2}{*}{9} & \multirow[t]{2}{*}{54} & \multirow[t]{2}{*}{1.4} & Left & No disease & No disease \\
\hline & & & Right & No disease & No disease \\
\hline \multirow[t]{2}{*}{10} & \multirow[t]{2}{*}{53} & \multirow[t]{2}{*}{1.9} & Left & $\begin{array}{c}\text { Minor } \\
\text { calcification }\end{array}$ & $\begin{array}{c}\text { Minor } \\
\text { calcification }\end{array}$ \\
\hline & & & Right & $\begin{array}{c}\text { Minor } \\
\text { calcification }\end{array}$ & $\begin{array}{c}\text { Minor } \\
\text { calcification }\end{array}$ \\
\hline \multirow[t]{2}{*}{11} & \multirow[t]{2}{*}{70} & \multirow[t]{2}{*}{1.3} & Left & $\begin{array}{c}\text { Minor } \\
\text { calcification }\end{array}$ & $\begin{array}{c}\text { Minor } \\
\text { calcification }\end{array}$ \\
\hline & & & Right & $\begin{array}{c}\text { Minor } \\
\text { calcification }\end{array}$ & $\begin{array}{c}\text { Minor } \\
\text { calcification }\end{array}$ \\
\hline \multirow[t]{2}{*}{12} & 77 & 1.2 & Left & $\begin{array}{c}\text { Minor } \\
\text { calcification }\end{array}$ & $\begin{array}{c}\text { Minor } \\
\text { calcification }\end{array}$ \\
\hline & & & Right & $\begin{array}{c}\text { Minor } \\
\text { calcification }\end{array}$ & $\begin{array}{c}\text { Minor } \\
\text { calcification }\end{array}$ \\
\hline
\end{tabular}


Table A2.1 continued.

\begin{tabular}{|c|c|c|c|c|c|}
\hline $\begin{array}{l}\text { Patient } \\
\text { Number }\end{array}$ & $\begin{array}{c}\text { Age } \\
\text { (Years) }\end{array}$ & $\begin{array}{c}\text { Maximum } \\
\text { Abdominal } \\
\text { Aorta Diameter } \\
(\mathbf{c m}) \\
\end{array}$ & Leg & $\begin{array}{l}\text { Iliac Artery } \\
\text { Disease State }\end{array}$ & $\begin{array}{c}\text { Femoro-Popliteal } \\
\text { Disease State }\end{array}$ \\
\hline \multirow[t]{2}{*}{13} & \multirow[t]{2}{*}{57} & \multirow[t]{2}{*}{1.3} & Left & $\begin{array}{c}\text { Minor } \\
\text { calcification }\end{array}$ & $\begin{array}{c}\text { Minor } \\
\text { calcification }\end{array}$ \\
\hline & & & Right & $\begin{array}{c}\text { Minor } \\
\text { calcification }\end{array}$ & $\begin{array}{c}\text { Minor } \\
\text { calcification }\end{array}$ \\
\hline \multirow[t]{2}{*}{14} & \multirow[t]{2}{*}{80} & \multirow[t]{2}{*}{1.7} & Left & $\begin{array}{c}\text { Minor } \\
\text { calcification } \\
\end{array}$ & $\begin{array}{c}\text { Minor } \\
\text { calcification } \\
\end{array}$ \\
\hline & & & Right & $\begin{array}{c}\text { Minor } \\
\text { calcification }\end{array}$ & $\begin{array}{c}\text { Minor } \\
\text { calcification }\end{array}$ \\
\hline \multirow[t]{2}{*}{15} & \multirow[t]{2}{*}{72} & \multirow[t]{2}{*}{1.7} & Left & $\begin{array}{c}\text { Minor } \\
\text { calcification } \\
\end{array}$ & $\begin{array}{c}\text { Minor } \\
\text { calcification }\end{array}$ \\
\hline & & & Right & $\begin{array}{c}\text { Minor } \\
\text { calcification }\end{array}$ & $\begin{array}{c}\text { Minor } \\
\text { calcification }\end{array}$ \\
\hline \multirow[t]{2}{*}{16} & \multirow[t]{2}{*}{81} & \multirow[t]{2}{*}{1.8} & Left & $\begin{array}{c}\text { Minor } \\
\text { calcification } \\
\end{array}$ & $\begin{array}{c}\text { Minor } \\
\text { calcification }\end{array}$ \\
\hline & & & Right & $\begin{array}{c}\text { Minor } \\
\text { calcification }\end{array}$ & $\begin{array}{c}\text { Minor } \\
\text { calcification }\end{array}$ \\
\hline \multirow[t]{2}{*}{17} & \multirow[t]{2}{*}{83} & \multirow[t]{2}{*}{1.5} & Left & No disease & No disease \\
\hline & & & Right & No disease & No disease \\
\hline \multirow[t]{2}{*}{18} & \multirow[t]{2}{*}{61} & \multirow[t]{2}{*}{1.4} & Left & No disease & No disease \\
\hline & & & Right & No disease & No disease \\
\hline \multirow[t]{2}{*}{19} & \multirow[t]{2}{*}{74} & \multirow[t]{2}{*}{1.4} & Left & $\begin{array}{c}\text { Minor } \\
\text { calcification } \\
\end{array}$ & $\begin{array}{c}\text { Minor } \\
\text { calcification } \\
\end{array}$ \\
\hline & & & Right & $\begin{array}{c}\text { Minor } \\
\text { calcification }\end{array}$ & $\begin{array}{c}\text { Minor } \\
\text { calcification }\end{array}$ \\
\hline \multirow[t]{2}{*}{20} & \multirow[t]{2}{*}{69} & \multirow[t]{2}{*}{1.5} & Left & $\begin{array}{c}\text { Minor } \\
\text { calcification }\end{array}$ & $\begin{array}{c}\text { Minor } \\
\text { calcification }\end{array}$ \\
\hline & & & Right & $\begin{array}{c}\text { Minor } \\
\text { calcification }\end{array}$ & $\begin{array}{c}\text { Minor } \\
\text { calcification }\end{array}$ \\
\hline \multirow[t]{2}{*}{21} & \multirow[t]{2}{*}{92} & \multirow[t]{2}{*}{1.1} & Left & $\begin{array}{c}\text { Minor } \\
\text { calcification }\end{array}$ & $\begin{array}{c}\text { Minor } \\
\text { calcification }\end{array}$ \\
\hline & & & Right & $\begin{array}{c}\text { Minor } \\
\text { calcification }\end{array}$ & $\begin{array}{c}\text { Minor } \\
\text { calcification }\end{array}$ \\
\hline \multirow[t]{2}{*}{22} & \multirow[t]{2}{*}{67} & \multirow[t]{2}{*}{1.5} & Left & $\begin{array}{c}\text { Minor } \\
\text { calcification }\end{array}$ & $\begin{array}{c}\text { Minor } \\
\text { calcification }\end{array}$ \\
\hline & & & Right & $\begin{array}{c}\text { Minor } \\
\text { calcification } \\
\end{array}$ & $\begin{array}{c}\text { Minor } \\
\text { calcification }\end{array}$ \\
\hline \multirow[t]{2}{*}{23} & \multirow[t]{2}{*}{58} & \multirow[t]{2}{*}{2.1} & Left & $\begin{array}{c}\text { Minor } \\
\text { calcification }\end{array}$ & $\begin{array}{c}\text { Minor } \\
\text { calcification }\end{array}$ \\
\hline & & & Right & $\begin{array}{c}\text { Minor } \\
\text { calcification }\end{array}$ & $\begin{array}{c}\text { Minor } \\
\text { calcification }\end{array}$ \\
\hline \multirow[t]{2}{*}{24} & 65 & 1.8 & Left & $\begin{array}{c}\text { Minor } \\
\text { calcification }\end{array}$ & $\begin{array}{c}\text { Minor } \\
\text { calcification }\end{array}$ \\
\hline & & & Right & $\begin{array}{c}\text { Minor } \\
\text { calcification }\end{array}$ & $\begin{array}{c}\text { Minor } \\
\text { calcification }\end{array}$ \\
\hline
\end{tabular}


Table A2.1 continued.

\begin{tabular}{|c|c|c|c|c|c|}
\hline $\begin{array}{l}\text { Patient } \\
\text { Number }\end{array}$ & $\begin{array}{c}\text { Age } \\
\text { (Years) }\end{array}$ & $\begin{array}{c}\text { Maximum } \\
\text { Abdominal } \\
\text { Aorta Diameter } \\
(\mathrm{cm}) \\
\end{array}$ & Leg & $\begin{array}{c}\text { Iliac Artery } \\
\text { Disease State }\end{array}$ & $\begin{array}{l}\text { Femoro-Popliteal } \\
\text { Disease State }\end{array}$ \\
\hline \multirow[t]{2}{*}{25} & \multirow[t]{2}{*}{75} & \multirow[t]{2}{*}{1.2} & Left & $\begin{array}{c}\text { Minor } \\
\text { calcification }\end{array}$ & $\begin{array}{c}\text { Minor } \\
\text { calcification }\end{array}$ \\
\hline & & & Right & $\begin{array}{c}\text { Minor } \\
\text { calcification }\end{array}$ & $\begin{array}{c}\text { Minor } \\
\text { calcification }\end{array}$ \\
\hline \multirow[t]{2}{*}{26} & \multirow[t]{2}{*}{50} & \multirow[t]{2}{*}{1.6} & Left & $\begin{array}{c}\text { Minor } \\
\text { calcification }\end{array}$ & $\begin{array}{c}\text { Minor } \\
\text { calcification }\end{array}$ \\
\hline & & & Right & $\begin{array}{c}\text { Minor } \\
\text { calcification }\end{array}$ & $\begin{array}{c}\text { Minor } \\
\text { calcification }\end{array}$ \\
\hline \multirow[t]{2}{*}{27} & \multirow[t]{2}{*}{87} & \multirow[t]{2}{*}{1.2} & Left & $\begin{array}{c}\text { Minor } \\
\text { calcification }\end{array}$ & $\begin{array}{c}\text { Minor } \\
\text { calcification }\end{array}$ \\
\hline & & & Right & $\begin{array}{c}\text { Minor } \\
\text { calcification }\end{array}$ & $\begin{array}{c}\text { Minor } \\
\text { calcification }\end{array}$ \\
\hline \multirow[t]{2}{*}{28} & \multirow[t]{2}{*}{77} & \multirow[t]{2}{*}{2.2} & Left & $\begin{array}{c}\text { Minor } \\
\text { calcification }\end{array}$ & $\begin{array}{c}\text { Minor } \\
\text { calcification }\end{array}$ \\
\hline & & & Right & $\begin{array}{c}\text { Minor } \\
\text { calcification } \\
\end{array}$ & $\begin{array}{c}\text { Minor } \\
\text { calcification } \\
\end{array}$ \\
\hline \multirow[t]{2}{*}{29} & \multirow[t]{2}{*}{83} & \multirow[t]{2}{*}{1.7} & Left & $\begin{array}{c}\text { Minor } \\
\text { calcification }\end{array}$ & $\begin{array}{c}\text { Minor } \\
\text { calcification }\end{array}$ \\
\hline & & & Right & $\begin{array}{c}\text { Minor } \\
\text { calcification }\end{array}$ & $\begin{array}{c}\text { Minor } \\
\text { calcification }\end{array}$ \\
\hline \multirow[t]{2}{*}{30} & \multirow[t]{2}{*}{56} & \multirow[t]{2}{*}{1.6} & Left & $\begin{array}{c}\text { Minor } \\
\text { calcification }\end{array}$ & $\begin{array}{c}\text { Minor } \\
\text { calcification } \\
\end{array}$ \\
\hline & & & Right & $\begin{array}{c}\text { Minor } \\
\text { calcification }\end{array}$ & $\begin{array}{c}\text { Minor } \\
\text { calcification }\end{array}$ \\
\hline
\end{tabular}


TableA2.2: Patient Data Summary for Normal Test Group

\begin{tabular}{|c|c|c|c|c|c|}
\hline $\begin{array}{l}\text { Patient } \\
\text { Number }\end{array}$ & $\begin{array}{c}\text { Age } \\
\text { (Years) }\end{array}$ & $\begin{array}{c}\text { Maximum } \\
\text { Abdominal } \\
\text { Aorta Diameter } \\
(\mathbf{c m}) \\
\end{array}$ & Leg & $\begin{array}{l}\text { Iliac Artery } \\
\text { Disease State }\end{array}$ & $\begin{array}{c}\text { Femoro-Popliteal } \\
\text { Disease State }\end{array}$ \\
\hline \multirow{2}{*}{1} & \multirow{2}{*}{73} & \multirow{2}{*}{1.5} & Left & $\begin{array}{c}\text { Minor } \\
\text { calcification }\end{array}$ & $\begin{array}{c}\text { Minor } \\
\text { calcification }\end{array}$ \\
\hline & & & Right & $\begin{array}{c}\text { Minor } \\
\text { calcification } \\
\end{array}$ & $\begin{array}{c}\text { Minor } \\
\text { calcification }\end{array}$ \\
\hline \multirow{2}{*}{2} & \multirow{2}{*}{58} & \multirow{2}{*}{2.0} & Left & $\begin{array}{c}\text { Minor } \\
\text { calcification }\end{array}$ & $\begin{array}{c}\text { Minor } \\
\text { calcification }\end{array}$ \\
\hline & & & Right & $\begin{array}{c}\text { Minor } \\
\text { calcification }\end{array}$ & $\begin{array}{c}\text { Minor } \\
\text { calcification }\end{array}$ \\
\hline \multirow{2}{*}{3} & \multirow{2}{*}{76} & \multirow{2}{*}{1.7} & Left & $\begin{array}{c}\text { Minor } \\
\text { calcification }\end{array}$ & $\begin{array}{c}\text { Minor } \\
\text { calcification }\end{array}$ \\
\hline & & & Right & $\begin{array}{c}\text { Minor } \\
\text { calcification }\end{array}$ & $\begin{array}{c}\text { Minor } \\
\text { calcification }\end{array}$ \\
\hline \multirow{2}{*}{4} & \multirow{2}{*}{78} & \multirow{2}{*}{1.6} & Left & $\begin{array}{c}\text { Minor } \\
\text { calcification }\end{array}$ & $\begin{array}{c}\text { Minor } \\
\text { calcification }\end{array}$ \\
\hline & & & Right & $\begin{array}{c}\text { Minor } \\
\text { calcification }\end{array}$ & $\begin{array}{c}\text { Minor } \\
\text { calcification }\end{array}$ \\
\hline \multirow{2}{*}{5} & \multirow{2}{*}{50} & \multirow{2}{*}{2.0} & Left & No disease & No disease \\
\hline & & & Right & No disease & No disease \\
\hline \multirow{2}{*}{6} & \multirow{2}{*}{76} & \multirow{2}{*}{1.7} & Left & $\begin{array}{c}\text { Minor } \\
\text { calcification } \\
\end{array}$ & $\begin{array}{c}\text { Minor } \\
\text { calcification }\end{array}$ \\
\hline & & & Right & $\begin{array}{c}\text { Minor } \\
\text { calcification }\end{array}$ & $\begin{array}{c}\text { Minor } \\
\text { calcification }\end{array}$ \\
\hline \multirow{2}{*}{7} & \multirow{2}{*}{72} & \multirow{2}{*}{1.4} & Left & $\begin{array}{c}\text { Minor } \\
\text { calcification }\end{array}$ & $\begin{array}{c}\text { Minor } \\
\text { calcification }\end{array}$ \\
\hline & & & Right & $\begin{array}{c}\text { Minor } \\
\text { calcification }\end{array}$ & $\begin{array}{c}\text { Minor } \\
\text { calcification } \\
\end{array}$ \\
\hline \multirow{2}{*}{8} & \multirow{2}{*}{83} & \multirow{2}{*}{2.2} & Left & $\begin{array}{c}\text { Minor } \\
\text { calcification }\end{array}$ & $\begin{array}{c}\text { Minor } \\
\text { calcification }\end{array}$ \\
\hline & & & Right & $\begin{array}{c}\text { Minor } \\
\text { calcification }\end{array}$ & $\begin{array}{c}\text { Minor } \\
\text { calcification }\end{array}$ \\
\hline \multirow[t]{2}{*}{9} & \multirow[t]{2}{*}{73} & \multirow[t]{2}{*}{2.3} & Left & $\begin{array}{c}\text { Minor } \\
\text { calcification }\end{array}$ & $\begin{array}{c}\text { Minor } \\
\text { calcification }\end{array}$ \\
\hline & & & Right & $\begin{array}{c}\text { Minor } \\
\text { calcification }\end{array}$ & $\begin{array}{c}\text { Minor } \\
\text { calcification }\end{array}$ \\
\hline \multirow[t]{2}{*}{10} & \multirow[t]{2}{*}{69} & \multirow[t]{2}{*}{1.7} & Left & $\begin{array}{c}\text { Minor } \\
\text { calcification }\end{array}$ & $\begin{array}{c}\text { Minor } \\
\text { calcification }\end{array}$ \\
\hline & & & Right & $\begin{array}{c}\text { Minor } \\
\text { calcification }\end{array}$ & $\begin{array}{c}\text { Minor } \\
\text { calcification }\end{array}$ \\
\hline \multirow[t]{2}{*}{11} & \multirow[t]{2}{*}{72} & \multirow[t]{2}{*}{1.8} & Left & $\begin{array}{c}\text { Minor } \\
\text { calcification }\end{array}$ & $\begin{array}{c}\text { Minor } \\
\text { calcification }\end{array}$ \\
\hline & & & Right & $\begin{array}{c}\text { Minor } \\
\text { calcification }\end{array}$ & $\begin{array}{c}\text { Minor } \\
\text { calcification }\end{array}$ \\
\hline
\end{tabular}


Appendix 3: Patient Data Summary for AAA Patient Groups with Significant

\section{Atherosclerotic Disease}

Table A3.1: Patient Data Summary for AAA with Significant Femoro-Popliteal

Disease Study Group

\begin{tabular}{|c|c|c|c|c|c|c|c|c|}
\hline $\begin{array}{l}\text { Patient } \\
\text { Number }\end{array}$ & $\begin{array}{c}\text { Age } \\
\text { (Years) }\end{array}$ & $\begin{array}{c}\text { Maximum } \\
\text { Abdominal } \\
\text { Aorta } \\
\text { Diameter } \\
(\mathrm{cm}) \\
\end{array}$ & ILT & $\begin{array}{l}\text { Residual } \\
\text { Lumen } \\
\text { (cm) }\end{array}$ & Leg & $\begin{array}{l}\text { Iliac Artery } \\
\text { Disease } \\
\text { State }\end{array}$ & $\begin{array}{c}\text { Femoro- } \\
\text { Popliteal } \\
\text { Disease State }\end{array}$ & $\begin{array}{c}\text { Additional } \\
\text { Aneurysmal } \\
\text { Disease }\end{array}$ \\
\hline \multirow[b]{2}{*}{1} & \multirow[b]{2}{*}{71} & \multirow[b]{2}{*}{3.0} & \multirow[b]{2}{*}{ No } & \multirow[b]{2}{*}{-} & Left & $\begin{array}{l}\text { Minor } \\
\text { calcification }\end{array}$ & $\begin{array}{l}\text { Tight proximal } \\
\text { SFA stenosis. } \\
\text { Distal SFA } \\
\text { short segment } \\
\text { occlusion }\end{array}$ & No \\
\hline & & & & & Right & $\begin{array}{l}\text { Minor } \\
\text { calcification }\end{array}$ & $\begin{array}{c}2 \mathrm{~cm} 50 \% \\
\text { SFA origin } \\
\text { stenosis. Distal } \\
\text { SFA short } \\
\text { segment } \\
\text { occlusion. }\end{array}$ & No \\
\hline \multirow[t]{2}{*}{2} & \multirow[t]{2}{*}{81} & \multirow[t]{2}{*}{3.9} & \multirow[t]{2}{*}{ No } & \multirow[t]{2}{*}{-} & Left & $\begin{array}{l}\text { Minor } \\
\text { calcification }\end{array}$ & $\begin{array}{l}\text { Short segment } \\
\text { mid SFA } \\
\text { occlusion }\end{array}$ & No \\
\hline & & & & & Right & $\begin{array}{c}\text { Minor } \\
\text { calcification }\end{array}$ & $\begin{array}{c}\text { Minor } \\
\text { calcification }\end{array}$ & No \\
\hline \multirow{2}{*}{3} & \multirow{2}{*}{55} & \multirow{2}{*}{3.6} & \multirow{2}{*}{ No } & \multirow[b]{2}{*}{ - } & Left & $\begin{array}{c}\text { Minor } \\
\text { calcification }\end{array}$ & $\begin{array}{l}\text { SFA occluded } \\
\text { from origin. } \\
\text { Adductor level } \\
\text { reconstitution }\end{array}$ & No \\
\hline & & & & & Right & $\begin{array}{l}\text { Minor } \\
\text { calcification }\end{array}$ & $\begin{array}{l}\text { SFA occluded } \\
\text { from origin. } \\
\text { Distal SFA } \\
\text { reconstitution }\end{array}$ & No \\
\hline \multirow[t]{2}{*}{4} & \multirow[t]{2}{*}{74} & \multirow[t]{2}{*}{3.7} & \multirow[t]{2}{*}{ No } & \multirow[t]{2}{*}{ - } & Left & $\begin{array}{c}\text { Minor } \\
\text { calcification }\end{array}$ & $\begin{array}{c}\text { Short segment } \\
\text { SFA origin } \\
\text { occlusion }\end{array}$ & No \\
\hline & & & & & Right & $\begin{array}{c}\text { Minor } \\
\text { calcification }\end{array}$ & $\begin{array}{c}\text { Distal SFA } \\
\text { occlusion }\end{array}$ & No \\
\hline \multirow{2}{*}{5} & \multirow{2}{*}{78} & \multirow{2}{*}{4.6} & \multirow{2}{*}{ Yes } & \multirow{2}{*}{2.8} & Left & $\begin{array}{c}\text { Minor } \\
\text { calcification }\end{array}$ & $\begin{array}{l}\text { SFA occluded } \\
\text { from origin. } \\
\text { Distal SFA } \\
\text { reconstitution }\end{array}$ & No \\
\hline & & & & & Right & $\begin{array}{c}\text { Minor } \\
\text { calcification }\end{array}$ & $\begin{array}{c}\text { Mid SFA } \\
\text { occlusion with } \\
\text { popliteal } \\
\text { reconstitution }\end{array}$ & No \\
\hline
\end{tabular}


Table A3.1 continued

\begin{tabular}{|c|c|c|c|c|c|c|c|c|}
\hline $\begin{array}{l}\text { Patient } \\
\text { Number }\end{array}$ & $\begin{array}{c}\text { Age } \\
\text { (Years) }\end{array}$ & $\begin{array}{l}\text { Maximum } \\
\text { Abdominal } \\
\text { Aorta } \\
\text { Diameter } \\
\text { (cm) } \\
\end{array}$ & ILT & $\begin{array}{l}\text { Residual } \\
\text { Lumen } \\
\text { (cm) }\end{array}$ & Leg & $\begin{array}{l}\text { Iliac Artery } \\
\text { Disease } \\
\text { State }\end{array}$ & $\begin{array}{c}\text { Femoro- } \\
\text { Popliteal } \\
\text { Disease State }\end{array}$ & $\begin{array}{c}\text { Additional } \\
\text { Aneurysmal } \\
\text { Disease }\end{array}$ \\
\hline \multirow[t]{2}{*}{6} & \multirow[t]{2}{*}{71} & \multirow[t]{2}{*}{4.9} & \multirow[t]{2}{*}{ Yes } & \multirow[t]{2}{*}{4.0} & Left & $\begin{array}{l}\text { Minor } \\
\text { calcification }\end{array}$ & $\begin{array}{l}\text { Tight SFA } \\
\text { origin and mid } \\
\text { SFA stenosis. }\end{array}$ & No \\
\hline & & & & & Right & $\begin{array}{c}\text { Minor } \\
\text { calcification }\end{array}$ & $\begin{array}{c}\sim 50 \% \text { mid } \\
\text { SFA stenosis }\end{array}$ & No \\
\hline \multirow[t]{2}{*}{7} & \multirow[t]{2}{*}{70} & \multirow[t]{2}{*}{3.7} & \multirow[t]{2}{*}{ Yes } & \multirow[t]{2}{*}{2.1} & Left & $\begin{array}{c}\text { Minor } \\
\text { calcification }\end{array}$ & $\begin{array}{l}\text { SFA occluded } \\
\text { from just } \\
\text { distal to the } \\
\text { origin with } \\
\text { popliteal } \\
\text { reconstitution }\end{array}$ & No \\
\hline & & & & & Right & $\begin{array}{l}\text { Minor } \\
\text { calcification }\end{array}$ & $\begin{array}{c}\text { Extensive } \\
\text { calcification } \\
\text { with several } \\
\text { moderate SFA } \\
\text { stenoses } \\
\end{array}$ & No \\
\hline \multirow[t]{2}{*}{8} & \multirow[t]{2}{*}{81} & \multirow[t]{2}{*}{5.0} & \multirow[t]{2}{*}{ Yes } & \multirow[t]{2}{*}{4.0} & Left & $\begin{array}{c}\text { Minor } \\
\text { calcification }\end{array}$ & $\begin{array}{c}\text { Moderate } \\
\text { diffuse } \\
\text { calcification } \\
\text { throughout } \\
\text { with a tight } \\
\text { mid popliteal } \\
\text { stenosis } \\
\end{array}$ & No \\
\hline & & & & & Right & $\begin{array}{l}\text { Minor } \\
\text { calcification }\end{array}$ & $\begin{array}{c}\text { Diffuse } \\
\text { calcification } \\
\text { throughout } \\
\text { Several } \sim 50 \% \\
\text { SFA stenoses } \\
\end{array}$ & No \\
\hline \multirow[t]{2}{*}{9} & \multirow[t]{2}{*}{67} & \multirow[t]{2}{*}{5.1} & \multirow{2}{*}{ Yes } & \multirow[t]{2}{*}{4.0} & Left & $\begin{array}{l}\text { Minor } \\
\text { calcification }\end{array}$ & $\begin{array}{l}\text { SFA occluded } \\
\text { from origin. } \\
\text { Adductor level } \\
\text { reconstitution }\end{array}$ & No \\
\hline & & & & & Right & $\begin{array}{l}\text { Minor } \\
\text { calcification }\end{array}$ & $\begin{array}{l}\text { SFA occluded } \\
\text { from the origin } \\
\text { with popliteal } \\
\text { reconstitution }\end{array}$ & No \\
\hline \multirow[t]{2}{*}{10} & \multirow[t]{2}{*}{76} & \multirow[t]{2}{*}{5.8} & \multirow[t]{2}{*}{ No } & \multirow[t]{2}{*}{-} & Left & $\begin{array}{c}\text { Minor } \\
\text { calcification }\end{array}$ & $\begin{array}{c}\text { CFA } \\
\text { moderately } \\
\text { calcified. SFA } \\
\text { occluded from } \\
\text { origin } \\
\end{array}$ & No \\
\hline & & & & & Right & $\begin{array}{c}\text { Minor } \\
\text { calcification }\end{array}$ & $\begin{array}{c}\text { Diffuse } \\
\text { calcification }\end{array}$ & No \\
\hline
\end{tabular}


Table A3.1 continued

\begin{tabular}{|c|c|c|c|c|c|c|c|c|}
\hline $\begin{array}{l}\text { Patient } \\
\text { Number }\end{array}$ & $\begin{array}{c}\text { Age } \\
\text { (Years) }\end{array}$ & $\begin{array}{c}\text { Maximum } \\
\text { Abdominal } \\
\text { Aorta } \\
\text { Diameter } \\
(\mathbf{c m}) \\
\end{array}$ & ILT & $\begin{array}{c}\text { Residual } \\
\text { Lumen } \\
\text { (cm) }\end{array}$ & Leg & $\begin{array}{l}\text { Iliac Artery } \\
\text { Disease } \\
\text { State }\end{array}$ & $\begin{array}{c}\text { Femoro- } \\
\text { Popliteal } \\
\text { Disease State }\end{array}$ & $\begin{array}{c}\text { Additional } \\
\text { Aneurysmal } \\
\text { Disease }\end{array}$ \\
\hline \multirow[t]{2}{*}{11} & \multirow[t]{2}{*}{62} & \multirow[t]{2}{*}{3.3} & \multirow[t]{2}{*}{ Yes } & \multirow[t]{2}{*}{1.7} & Left & $\begin{array}{c}\text { Minor } \\
\text { calcification }\end{array}$ & $\begin{array}{c}\text { Tight distal } \\
\text { SFA stenosis }\end{array}$ & No \\
\hline & & & & & Right & $\begin{array}{c}\text { Minor } \\
\text { calcification }\end{array}$ & $\begin{array}{c}\text { Minor } \\
\text { calcification }\end{array}$ & No \\
\hline \multirow[t]{2}{*}{12} & \multirow[t]{2}{*}{82} & \multirow[t]{2}{*}{6.0} & \multirow[t]{2}{*}{ Yes } & \multirow[t]{2}{*}{2.5} & Left & $\begin{array}{c}\text { Minor } \\
\text { calcification }\end{array}$ & $\begin{array}{c}\text { Minor } \\
\text { calcification }\end{array}$ & No \\
\hline & & & & & Right & $\begin{array}{l}\text { Minor } \\
\text { calcification }\end{array}$ & $\begin{array}{l}\text { SFA occludes } \\
\sim 15 \mathrm{~cm} \text { from } \\
\text { the origin } \\
\text { with } \\
\text { adductor } \\
\text { reconstitution }\end{array}$ & $\begin{array}{c}3.6 \mathrm{~cm} \text { CIA } \\
\text { aneurysm }\end{array}$ \\
\hline \multirow[t]{2}{*}{13} & \multirow[t]{2}{*}{76} & \multirow[t]{2}{*}{6.3} & \multirow[t]{2}{*}{ No } & \multirow[t]{2}{*}{-} & Left & $\begin{array}{l}\text { Minor } \\
\text { calcification }\end{array}$ & $\begin{array}{c}\text { Diffuse } \\
\text { disease with } \\
\text { several } \\
\text { moderate } \\
\text { SFA stenoses }\end{array}$ & $\begin{array}{c}2.4 \mathrm{~cm} \mathrm{CIA} \\
\text { aneurysm }\end{array}$ \\
\hline & & & & & Right & $\begin{array}{l}\text { Minor } \\
\text { calcification }\end{array}$ & $\begin{array}{c}\text { Diffuse } \\
\text { disease with } \\
\text { several } \\
\text { moderate } \\
\text { SFA stenoses }\end{array}$ & $\begin{array}{l}2.0 \mathrm{~cm} \mathrm{CIA} \\
\text { aneurysm }\end{array}$ \\
\hline \multirow[t]{2}{*}{14} & \multirow[t]{2}{*}{80} & \multirow[t]{2}{*}{3.9} & \multirow[t]{2}{*}{ No } & \multirow[t]{2}{*}{-} & Left & $\begin{array}{l}\text { Minor } \\
\text { calcification }\end{array}$ & $\begin{array}{l}\text { SFA occluded } \\
\text { from origin } \\
\text { with popliteal } \\
\text { reconstitution }\end{array}$ & No \\
\hline & & & & & Right & $\begin{array}{l}\text { Minor } \\
\text { calcification }\end{array}$ & $\begin{array}{l}\text { SFA occluded } \\
\text { from origin } \\
\text { with popliteal } \\
\text { reconstitution }\end{array}$ & No \\
\hline \multirow[t]{2}{*}{15} & \multirow[t]{2}{*}{85} & \multirow[t]{2}{*}{5.6} & \multirow[t]{2}{*}{ No } & \multirow[t]{2}{*}{ - } & Left & $\begin{array}{c}\text { Minor } \\
\text { calcification }\end{array}$ & $\begin{array}{c}50 \% \text { mid } \\
\text { SFA stenosis }\end{array}$ & No \\
\hline & & & & & Right & $\begin{array}{c}\text { Minor } \\
\text { calcification }\end{array}$ & $\begin{array}{l}50-70 \% \text { mid } \\
\text { SFA stenosis }\end{array}$ & No \\
\hline
\end{tabular}


Table A3.2: Patient Data Summary for AAA with Significant Femoro-Popliteal Disease Test Group

\begin{tabular}{|c|c|c|c|c|c|c|c|c|}
\hline $\begin{array}{l}\text { Patient } \\
\text { Number }\end{array}$ & $\begin{array}{c}\text { Age } \\
\text { (Years) }\end{array}$ & $\begin{array}{l}\text { Maximum } \\
\text { Abdominal } \\
\text { Aorta } \\
\text { Diameter } \\
\text { (cm) } \\
\end{array}$ & ILT & $\begin{array}{c}\text { Residual } \\
\text { Lumen } \\
\text { (cm) }\end{array}$ & Leg & $\begin{array}{c}\text { Iliac Artery } \\
\text { Disease } \\
\text { State }\end{array}$ & $\begin{array}{c}\text { Femoro- } \\
\text { Popliteal } \\
\text { Disease State }\end{array}$ & $\begin{array}{c}\text { Additional } \\
\text { Aneurysmal } \\
\text { Disease }\end{array}$ \\
\hline \multirow{2}{*}{1} & \multirow{2}{*}{76} & \multirow{2}{*}{4.8} & \multirow{2}{*}{ Yes } & \multirow{2}{*}{2.5} & Left & $\begin{array}{c}\text { Minor } \\
\text { calcification }\end{array}$ & $\begin{array}{l}\text { Mid SFA } \\
\text { occlusion }\end{array}$ & No \\
\hline & & & & & Right & $\begin{array}{c}\text { Minor } \\
\text { calcification }\end{array}$ & $\begin{array}{l}\text { SFA occluded } \\
\text { from origin }\end{array}$ & No \\
\hline \multirow[t]{2}{*}{2} & \multirow[t]{2}{*}{91} & \multirow[t]{2}{*}{5.0} & \multirow[t]{2}{*}{ No } & \multirow[t]{2}{*}{-} & Left & $\begin{array}{c}\text { Minor } \\
\text { calcification }\end{array}$ & $\begin{array}{c}\text { Diffuse } \\
\text { disease } \\
\text { throughout } \\
\text { with a tight } \\
\text { proximal } \\
\text { popliteal } \\
\text { stenosis } \\
\end{array}$ & No \\
\hline & & & & & Right & $\begin{array}{c}\text { Minor } \\
\text { calcification }\end{array}$ & $\begin{array}{l}\text { SFA occludes } \\
\sim 10 \mathrm{~cm} \text { from } \\
\text { the origin } \\
\text { with } \\
\text { adductor } \\
\text { reconstitution }\end{array}$ & No \\
\hline \multirow{2}{*}{3} & \multirow{2}{*}{88} & \multirow{2}{*}{5.6} & \multirow{2}{*}{ No } & \multirow{2}{*}{-} & Left & $\begin{array}{l}\text { Minor } \\
\text { calcification }\end{array}$ & $\begin{array}{c}\text { SFA } \\
\text { narrowed } \\
\text { throughout } \\
\text { with several } \\
50-70 \% \\
\text { stenoses } \\
\end{array}$ & No \\
\hline & & & & & Right & $\begin{array}{c}\text { Minor } \\
\text { calcification }\end{array}$ & $\begin{array}{c}\text { SFA } \\
\text { narrowed } \\
\text { throughout } \\
\text { with several } \\
50-70 \% \\
\text { stenoses }\end{array}$ & No \\
\hline
\end{tabular}


Table A3.3: Patient Data Summary for AAA with Significant Iliac Disease Group

\begin{tabular}{|c|c|c|c|c|c|c|c|c|}
\hline $\begin{array}{l}\text { Patient } \\
\text { Number }\end{array}$ & $\begin{array}{c}\text { Age } \\
\text { (Years) }\end{array}$ & $\begin{array}{l}\text { Maximum } \\
\text { Abdominal } \\
\text { Aorta } \\
\text { Diameter } \\
\text { (cm) }\end{array}$ & ILT & $\begin{array}{c}\text { Residual } \\
\text { Lumen } \\
\text { (cm) }\end{array}$ & Leg & $\begin{array}{l}\text { Iliac Artery } \\
\text { Disease State }\end{array}$ & $\begin{array}{c}\text { Femoro- } \\
\text { Popliteal } \\
\text { Disease State }\end{array}$ & $\begin{array}{c}\text { Additional } \\
\text { Aneurysmal } \\
\text { Disease }\end{array}$ \\
\hline \multirow[t]{2}{*}{1} & \multirow[t]{2}{*}{76} & \multirow[t]{2}{*}{3.6} & \multirow[t]{2}{*}{ Yes } & \multirow[t]{2}{*}{1.9} & Left & $\begin{array}{l}\text { Tight EIA } \\
\text { stenosis }\end{array}$ & $\begin{array}{l}\text { Tight SFA } \\
\text { origin } \\
\text { stenosis. SFA } \\
\text { patent but } \\
\text { very diseased }\end{array}$ & No \\
\hline & & & & & Right & $\begin{array}{l}\text { CIA and EIA } \\
\text { occluded }\end{array}$ & $\begin{array}{l}\text { SFA occluded } \\
\text { from origin }\end{array}$ & No \\
\hline \multirow{2}{*}{2} & \multirow{2}{*}{76} & \multirow{2}{*}{4.8} & \multirow{2}{*}{ Yes } & \multirow{2}{*}{2.2} & Left & $\begin{array}{c}\text { CIA } \\
\text { occlusion }\end{array}$ & $\begin{array}{c}\text { Minor } \\
\text { calcification }\end{array}$ & No \\
\hline & & & & & Right & $\begin{array}{c}\text { Minor } \\
\text { calcification }\end{array}$ & $\begin{array}{c}\text { Minor } \\
\text { calcification }\end{array}$ & No \\
\hline \multirow[t]{2}{*}{3} & \multirow[t]{2}{*}{74} & \multirow[t]{2}{*}{4.9} & \multirow[t]{2}{*}{ Yes } & \multirow[t]{2}{*}{2.3} & Left & EIA stenosis & $\begin{array}{l}\text { SFA occluded } \\
\text { from the } \\
\text { origin with } \\
\text { popliteal } \\
\text { reconstitution }\end{array}$ & No \\
\hline & & & & & Right & $\begin{array}{c}\text { Minor } \\
\text { calcification }\end{array}$ & $\begin{array}{c}\text { SFA } \\
\text { moderately } \\
\text { narrowed } \\
\text { throughout }\end{array}$ & No \\
\hline \multirow[t]{2}{*}{4} & \multirow[t]{2}{*}{$\overline{73}$} & \multirow[t]{2}{*}{4.4} & \multirow[t]{2}{*}{ No } & \multirow[t]{2}{*}{-} & Left & $\begin{array}{c}\text { Tight EIA } \\
\text { origin and } \\
\text { distal EIA } \\
\text { stenosis }\end{array}$ & $\begin{array}{l}\text { SFA occluded } \\
\text { from origin }\end{array}$ & No \\
\hline & & & & & Right & $\begin{array}{c}\text { Moderate } \\
\text { EIA stenosis }\end{array}$ & $\begin{array}{l}\text { Tight distal } \\
\text { CFA stenosis. } \\
\text { SFA occluded } \\
\text { from origin }\end{array}$ & No \\
\hline \multirow[t]{2}{*}{5} & \multirow[t]{2}{*}{79} & \multirow[t]{2}{*}{6.3} & \multirow[t]{2}{*}{ Yes } & \multirow[t]{2}{*}{2.3} & Left & $\begin{array}{c}\text { CIA } \\
\text { occlusion } \\
\text { with EIA } \\
\text { reconstitution } \\
\text { via IIA } \\
\end{array}$ & $\begin{array}{l}\text { Diffuse } \\
\text { disease }\end{array}$ & No \\
\hline & & & & & Right & $\begin{array}{c}\text { Minor } \\
\text { calcification }\end{array}$ & $\begin{array}{l}\text { Diffuse } \\
\text { disease }\end{array}$ & No \\
\hline \multirow[t]{2}{*}{6} & \multirow[t]{2}{*}{74} & \multirow[t]{2}{*}{6.0} & \multirow[t]{2}{*}{ No } & \multirow[t]{2}{*}{-} & Left & EIA occluded & $\begin{array}{c}\text { CFA } \\
\text { occluded with } \\
\text { SFA origin } \\
\text { reconstitution }\end{array}$ & No \\
\hline & & & & & Right & $\begin{array}{c}\text { Tight EIA } \\
\text { stenosis }\end{array}$ & $\begin{array}{c}\text { Tight mid } \\
\text { SFA stenosis. } \\
\text { Distal SFA } \\
\text { occlusion }\end{array}$ & $\begin{array}{l}3.6 \mathrm{~cm} \text { CIA } \\
\text { aneurysm }\end{array}$ \\
\hline
\end{tabular}


Appendix 4: Patient Data Summary for Patient Groups with No AAA and

\section{Significant Atherosclerotic Disease}

Table A4.1: Patient Data Summary for No AAA with Significant Femoro-Popliteal Disease Study Group

\begin{tabular}{|c|c|c|c|c|c|}
\hline $\begin{array}{l}\text { Patient } \\
\text { Number }\end{array}$ & $\begin{array}{c}\text { Age } \\
\text { (Years) }\end{array}$ & $\begin{array}{c}\text { Maximum } \\
\text { Abdominal } \\
\text { Aorta Diameter } \\
(\mathbf{c m}) \\
\end{array}$ & Leg & $\begin{array}{l}\text { Iliac Artery } \\
\text { Disease State }\end{array}$ & $\begin{array}{l}\text { Femoro-Popliteal } \\
\text { Disease State }\end{array}$ \\
\hline \multirow{2}{*}{1} & \multirow{2}{*}{83} & \multirow{2}{*}{ 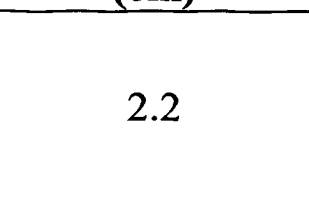 } & Left & $\begin{array}{c}\text { Minor } \\
\text { calcification }\end{array}$ & $\begin{array}{c}\text { Popliteal occlusion } \\
\text { at trifurcation }\end{array}$ \\
\hline & & & Right & $\begin{array}{c}\text { Minor } \\
\text { calcification }\end{array}$ & $\begin{array}{c}\text { Distal popliteal } \\
\text { occlusion }\end{array}$ \\
\hline \multirow{2}{*}{2} & \multirow{2}{*}{80} & \multirow{2}{*}{1.6} & Left & $\begin{array}{c}\text { Minor } \\
\text { calcification }\end{array}$ & $\begin{array}{c}\text { Diffuse disease } \\
\text { with several } \\
\text { moderate SFA } \\
\text { stenoses }\end{array}$ \\
\hline & & & Right & $\begin{array}{c}\text { Minor } \\
\text { calcification }\end{array}$ & $\begin{array}{c}\text { Diffuse disease } \\
\text { with several } \\
\text { moderate SFA } \\
\text { stenoses }\end{array}$ \\
\hline \multirow[t]{2}{*}{3} & \multirow[t]{2}{*}{81} & \multirow[t]{2}{*}{1.6} & Left & $\begin{array}{c}\text { Minor } \\
\text { calcification }\end{array}$ & $\begin{array}{c}\text { Diffuse disease } \\
\text { with at least one } \\
\text { moderate SFA } \\
\text { stenosis }\end{array}$ \\
\hline & & & Right & $\begin{array}{c}\text { Minor } \\
\text { calcification }\end{array}$ & $\begin{array}{c}\text { Diffuse disease } \\
\text { with tight mid SFA } \\
\text { stenosis }\end{array}$ \\
\hline \multirow[b]{2}{*}{4} & \multirow[b]{2}{*}{67} & \multirow[b]{2}{*}{1.7} & Left & $\begin{array}{c}\text { Minor } \\
\text { calcification }\end{array}$ & $\begin{array}{c}\text { Diffuse moderate } \\
\text { disease }\end{array}$ \\
\hline & & & Right & $\begin{array}{c}\text { Minor } \\
\text { calcification }\end{array}$ & $\begin{array}{c}\text { Diffuse disease } \\
\text { with } \sim 50-70 \% \\
\text { proximal SFA } \\
\text { stenosis }\end{array}$ \\
\hline \multirow[t]{2}{*}{5} & \multirow[t]{2}{*}{53} & \multirow[t]{2}{*}{1.5} & Left & $\begin{array}{c}\text { Minor } \\
\text { calcification }\end{array}$ & $\begin{array}{c}\text { Diffuse disease } \\
\text { with } \sim 50-70 \% \mathrm{mid} \\
\text { SFA stenosis } \\
\end{array}$ \\
\hline & & & Right & $\begin{array}{c}\text { Minor } \\
\text { calcification } \\
\end{array}$ & Minor calcification \\
\hline \multirow[b]{2}{*}{6} & \multirow[b]{2}{*}{90} & \multirow[b]{2}{*}{1.5} & Left & $\begin{array}{c}\text { Minor } \\
\text { calcification }\end{array}$ & $\begin{array}{l}\text { SFA occluded } \\
\text { from origin }\end{array}$ \\
\hline & & & Right & $\begin{array}{c}\text { Minor } \\
\text { calcification }\end{array}$ & $\begin{array}{l}\text { SFA occluded } \\
\text { from origin }\end{array}$ \\
\hline
\end{tabular}


Table A4.1 continued

\begin{tabular}{|c|c|c|c|c|c|}
\hline $\begin{array}{l}\text { Patient } \\
\text { Number }\end{array}$ & $\begin{array}{c}\text { Age } \\
\text { (Years) }\end{array}$ & $\begin{array}{c}\text { Maximum } \\
\text { Abdominal } \\
\text { Aorta Diameter } \\
(\mathrm{cm}) \\
\end{array}$ & Leg & $\begin{array}{l}\text { Iliac Artery } \\
\text { Disease State }\end{array}$ & $\begin{array}{c}\text { Femoro-Popliteal } \\
\text { Disease State }\end{array}$ \\
\hline \multirow{2}{*}{7} & \multirow{2}{*}{95} & \multirow{2}{*}{2.5} & Left & $\begin{array}{c}\text { Minor } \\
\text { calcification }\end{array}$ & $\begin{array}{c}\text { Very calcified SFA } \\
\text { with multiple tight } \\
\text { stenoses }\end{array}$ \\
\hline & & & Right & $\begin{array}{c}\text { Minor } \\
\text { calcification }\end{array}$ & $\begin{array}{c}\text { Very calcified SFA } \\
\text { with multiple tight } \\
\text { stenoses }\end{array}$ \\
\hline \multirow{2}{*}{8} & \multirow{2}{*}{50} & \multirow{2}{*}{1.6} & Left & $\begin{array}{c}\text { Minor } \\
\text { calcification }\end{array}$ & $\begin{array}{c}\text { Short segment } \\
\text { popliteal occlusion }\end{array}$ \\
\hline & & & Right & $\begin{array}{c}\text { Minor } \\
\text { calcification }\end{array}$ & Moderate disease \\
\hline \multirow{2}{*}{9} & \multirow{2}{*}{73} & \multirow{2}{*}{1.3} & Left & $\begin{array}{c}\text { Minor } \\
\text { calcification }\end{array}$ & $\begin{array}{c}\text { Two } 50-70 \% \text { SFA } \\
\text { stenoses }\end{array}$ \\
\hline & & & Right & $\begin{array}{c}\text { Minor } \\
\text { calcification }\end{array}$ & $\begin{array}{c}\text { At least two } 50- \\
70 \% \text { SFA stenoses }\end{array}$ \\
\hline \multirow{2}{*}{10} & \multirow{2}{*}{76} & \multirow{2}{*}{1.5} & Left & $\begin{array}{c}\text { Minor } \\
\text { calcification }\end{array}$ & $\begin{array}{c}\text { Very calcified SFA } \\
\text { with multiple tight } \\
\text { stenoses }\end{array}$ \\
\hline & & & Right & $\begin{array}{c}\text { Minor } \\
\text { calcification }\end{array}$ & $\begin{array}{c}\text { Very calcified SFA } \\
\text { with multiple tight } \\
\text { stenoses }\end{array}$ \\
\hline \multirow{2}{*}{11} & \multirow{2}{*}{76} & \multirow{2}{*}{1.3} & Left & $\begin{array}{c}\text { Minor } \\
\text { calcification }\end{array}$ & $\begin{array}{c}\text { Diffuse } \\
\text { calcification with } \\
\text { at least } 150-70 \% \\
\text { mid SFA stenosis }\end{array}$ \\
\hline & & & Right & $\begin{array}{c}\text { Minor } \\
\text { calcification }\end{array}$ & $\begin{array}{c}\text { Diffuse } \\
\text { calcification but no } \\
\text { significant focal } \\
\text { lesions }\end{array}$ \\
\hline \multirow{2}{*}{12} & \multirow{2}{*}{73} & \multirow{2}{*}{1.2} & Left & $\begin{array}{c}\text { Minor } \\
\text { calcification }\end{array}$ & $\begin{array}{l}\text { SFA occluded } \\
\text { from origin with } \\
\text { adductor level } \\
\text { reconstitution }\end{array}$ \\
\hline & & & Right & $\begin{array}{c}\text { Minor } \\
\text { calcification }\end{array}$ & $\begin{array}{c}\text { Distal CFA } \\
\text { occlusion with } \\
\text { proximal popliteal } \\
\text { reconstitution }\end{array}$ \\
\hline \multirow[t]{2}{*}{13} & \multirow[t]{2}{*}{96} & \multirow[t]{2}{*}{1.6} & Left & $\begin{array}{c}\text { Minor } \\
\text { calcification }\end{array}$ & $\begin{array}{c}\text { Diffuse disease } \\
\text { with distal SFA } \\
\text { occlusion } \\
\end{array}$ \\
\hline & & & Right & $\begin{array}{c}\text { Minor } \\
\text { calcification }\end{array}$ & $\begin{array}{l}\text { SFA occluded } \\
\text { from origin }\end{array}$ \\
\hline
\end{tabular}


Table A4.1 continued

\begin{tabular}{|c|c|c|c|c|c|}
\hline $\begin{array}{l}\text { Patient } \\
\text { Number }\end{array}$ & $\begin{array}{c}\text { Age } \\
\text { (Years) }\end{array}$ & $\begin{array}{c}\text { Maximum } \\
\text { Abdominal } \\
\text { Aorta Diameter } \\
(\mathbf{c m}) \\
\end{array}$ & Leg & $\begin{array}{l}\text { Iliac Artery } \\
\text { Disease State }\end{array}$ & $\begin{array}{c}\text { Femoro-Popliteal } \\
\text { Disease State }\end{array}$ \\
\hline \multirow{2}{*}{14} & \multirow{2}{*}{70} & \multirow{2}{*}{2.1} & Left & $\begin{array}{l}\text { Minor } \\
\text { calcification }\end{array}$ & $\begin{array}{c}\text { Diffuse } \\
\text { calcification with } \\
\text { multiple } 50-70 \% \\
\text { SFA stenoses } \\
\end{array}$ \\
\hline & & & Right & $\begin{array}{c}\text { Minor } \\
\text { calcification }\end{array}$ & $\begin{array}{c}\text { Diffuse } \\
\text { calcification with } \\
\text { multiple } 50-70 \% \\
\text { SFA stenoses } \\
\end{array}$ \\
\hline \multirow[t]{2}{*}{15} & \multirow[t]{2}{*}{65} & \multirow[t]{2}{*}{1.87} & Left & $\begin{array}{c}\text { Minor } \\
\text { calcification }\end{array}$ & $\begin{array}{c}\text { Diffuse } \\
\text { calcification with } \\
\text { at least } 150-70 \% \\
\text { mid SFA stenosis } \\
\end{array}$ \\
\hline & & & Right & $\begin{array}{c}\text { Minor } \\
\text { calcification }\end{array}$ & $\begin{array}{c}\text { Minor calcification } \\
\text { with SFA adductor } \\
\text { level occlusion }\end{array}$ \\
\hline \multirow[t]{2}{*}{16} & \multirow[t]{2}{*}{85} & \multirow[t]{2}{*}{1.3} & Left & $\begin{array}{c}\text { Minor } \\
\text { calcification }\end{array}$ & $\begin{array}{c}\text { Diffuse } \\
\text { calcification with } \\
\text { at least } 150-70 \% \\
\text { mid SFA stenosis } \\
\end{array}$ \\
\hline & & & Right & $\begin{array}{c}\text { Minor } \\
\text { calcification }\end{array}$ & $\begin{array}{c}\text { Diffuse } \\
\text { calcification with } \\
\text { at least } 150-70 \% \\
\text { mid SFA stenosis } \\
\end{array}$ \\
\hline \multirow[t]{2}{*}{17} & \multirow[t]{2}{*}{67} & \multirow[t]{2}{*}{2.0} & Left & $\begin{array}{c}\text { Minor } \\
\text { calcification }\end{array}$ & $\begin{array}{c}\text { Diffuse } \\
\text { calcification with } \\
\text { multiple } 50-70 \% \\
\text { SFA stenoses } \\
\end{array}$ \\
\hline & & & Right & $\begin{array}{c}\text { Minor } \\
\text { calcification }\end{array}$ & $\begin{array}{c}\text { Diffuse } \\
\text { calcification with } \\
\text { multiple } 50-70 \% \\
\text { SFA stenoses } \\
\end{array}$ \\
\hline \multirow[t]{2}{*}{18} & \multirow[t]{2}{*}{56} & \multirow[t]{2}{*}{1.2} & Left & $\begin{array}{c}\text { Minor } \\
\text { calcification }\end{array}$ & $\begin{array}{c}\text { SFA diffusely } \\
\text { narrowed } \\
\text { throughout }\end{array}$ \\
\hline & & & Right & $\begin{array}{c}\text { Minor } \\
\text { calcification }\end{array}$ & $\begin{array}{c}\text { Moderate } \\
\text { calcification }\end{array}$ \\
\hline
\end{tabular}


Table A4.2: Patient Data Summary for No AAA with Significant Femoro-Popliteal Disease test Group

\begin{tabular}{|c|c|c|c|c|c|}
\hline $\begin{array}{l}\text { Patient } \\
\text { Number }\end{array}$ & $\begin{array}{c}\text { Age } \\
\text { (Years) }\end{array}$ & $\begin{array}{c}\text { Maximum } \\
\text { Abdominal } \\
\text { Aorta Diameter } \\
\text { (cm) }\end{array}$ & Leg & $\begin{array}{l}\text { Iliac Artery } \\
\text { Disease State }\end{array}$ & $\begin{array}{c}\text { Femoro-Popliteal } \\
\text { Disease State }\end{array}$ \\
\hline \multirow[t]{2}{*}{1} & \multirow[t]{2}{*}{70} & \multirow[t]{2}{*}{2.0} & Left & $\begin{array}{l}\text { Minor } \\
\text { calcification }\end{array}$ & $\begin{array}{c}\text { Diffuse disease } \\
\text { with tight mid SFA } \\
\text { and adductor level } \\
\text { stenoses }\end{array}$ \\
\hline & & & Right & $\begin{array}{c}\text { Minor } \\
\text { calcification }\end{array}$ & $\begin{array}{c}\text { Diffuse disease } \\
\text { with tight distal } \\
\text { SFA stenosis }\end{array}$ \\
\hline \multirow{2}{*}{2} & \multirow{2}{*}{73} & \multirow{2}{*}{1.2} & Left & $\begin{array}{c}\text { Minor } \\
\text { calcification }\end{array}$ & Moderate disease \\
\hline & & & Right & $\begin{array}{c}\text { Minor } \\
\text { calcification }\end{array}$ & $\begin{array}{c}\text { Distal SFA } \\
\text { occlusion }\end{array}$ \\
\hline \multirow{2}{*}{3} & \multirow{2}{*}{75} & \multirow{2}{*}{1.6} & Left & $\begin{array}{c}\text { Minor } \\
\text { calcification }\end{array}$ & $\begin{array}{l}\text { Diffuse moderate } \\
\text { disease with } \\
\text { significant disease } \\
\text { at trifurcation }\end{array}$ \\
\hline & & & Right & $\begin{array}{c}\text { Minor } \\
\text { calcification }\end{array}$ & $\begin{array}{l}\text { Diffuse moderate } \\
\text { disease with } \\
\text { significant disease } \\
\text { at trifurcation }\end{array}$ \\
\hline \multirow{2}{*}{4} & \multirow{2}{*}{63} & \multirow{2}{*}{1.7} & Left & $\begin{array}{l}\text { Minor } \\
\text { calcification }\end{array}$ & $\begin{array}{c}\text { Diffuse disease } \\
\text { with multiple tight } \\
\text { SFA stenoses }\end{array}$ \\
\hline & & & Right & $\begin{array}{l}\text { Minor } \\
\text { calcification }\end{array}$ & $\begin{array}{c}\text { Diffuse disease } \\
\text { with multiple tight } \\
\text { SFA stenoses }\end{array}$ \\
\hline
\end{tabular}


Table A4.3: Patient Data Summary for No AAA with Significant Iliac Disease Group

\begin{tabular}{|c|c|c|c|c|c|}
\hline $\begin{array}{l}\text { Patient } \\
\text { Number }\end{array}$ & $\begin{array}{c}\text { Age } \\
\text { (Years) }\end{array}$ & $\begin{array}{c}\text { Maximum } \\
\text { Abdominal } \\
\text { Aorta } \\
\text { Diameter (cm) }\end{array}$ & Leg & $\begin{array}{l}\text { Iliac Artery } \\
\text { Disease State }\end{array}$ & $\begin{array}{l}\text { Femoro-Popliteal } \\
\text { Disease State }\end{array}$ \\
\hline \multirow[b]{2}{*}{1} & \multirow[b]{2}{*}{67} & \multirow[b]{2}{*}{1.7} & Left & CIA occlusion & Minor calcification \\
\hline & & & Right & $\begin{array}{c}\text { Minor } \\
\text { calcification }\end{array}$ & Minor calcification \\
\hline \multirow[t]{2}{*}{2} & \multirow[t]{2}{*}{71} & \multirow[t]{2}{*}{2.6} & Left & $\begin{array}{c}\text { Tight CIA } \\
\text { origin stenosis }\end{array}$ & $\begin{array}{c}\text { Distal CFA } \\
\text { occlusion with } \\
\text { proximal popliteal } \\
\text { reconstitution }\end{array}$ \\
\hline & & & Right & $\begin{array}{c}\text { Tight CIA } \\
\text { origin stenosis }\end{array}$ & $\begin{array}{l}\text { SFA occluded } \\
\text { from origin }\end{array}$ \\
\hline \multirow{2}{*}{3} & \multirow{2}{*}{51} & \multirow{2}{*}{1.7} & Left & $\begin{array}{c}\text { Minor } \\
\text { calcification }\end{array}$ & Minor calcification \\
\hline & & & Right & $\begin{array}{c}\text { Tight EIA } \\
\text { stenosis }\end{array}$ & Minor calcification \\
\hline \multirow{2}{*}{4} & \multirow{2}{*}{87} & \multirow{2}{*}{1.5} & Left & $\begin{array}{c}\text { Tight distal EIA } \\
\text { stenosis }\end{array}$ & $\begin{array}{l}\text { SFA occluded } \\
\text { from origin }\end{array}$ \\
\hline & & & Right & $\begin{array}{c}\text { Minor } \\
\text { calcification }\end{array}$ & $\begin{array}{l}\text { SFA occluded } \\
\text { from origin }\end{array}$ \\
\hline \multirow[t]{2}{*}{5} & \multirow[t]{2}{*}{75} & \multirow[t]{2}{*}{1.6} & Left & CIA occlusion & Mid SFA occlusion \\
\hline & & & Right & $\begin{array}{l}\text { Significant } \\
\text { distal CIA and } \\
\text { proximal EIA } \\
\text { disease }\end{array}$ & Mid SFA occlusion \\
\hline \multirow[t]{2}{*}{6} & \multirow[t]{2}{*}{52} & \multirow[t]{2}{*}{1.2} & Left & $\begin{array}{c}\text { Tight EIA } \\
\text { stenosis }\end{array}$ & Minor calcification \\
\hline & & & Right & $\begin{array}{c}\text { Minor } \\
\text { calcification }\end{array}$ & Minor calcification \\
\hline \multirow[t]{2}{*}{7} & \multirow[t]{2}{*}{85} & \multirow[t]{2}{*}{2.0} & Left & $\begin{array}{l}\text { Tight CIA } \\
\text { stenosis }\end{array}$ & Minor calcification \\
\hline & & & Right & $\begin{array}{c}\sim 50-70 \% \text { CIA } \\
\text { stenosis }\end{array}$ & Minor calcification \\
\hline
\end{tabular}




\section{Appendix 5: Explanation of Index Plot Format}

The levels for the indices calculated for the left and right CFA waveforms for each of the AAA and no AAA patients in the study and test groups are plotted in the format illustrated in Figure A1 below. In this example, the Maximum Down-Stroke Bandwidth Index levels are plotted for the $30 \mathrm{AAA}$ and 30 normal patients in the no significant atherosclerotic disease study groups. Due to 'font size limitation' there was not enough space on the horizontal axes to label both the left and right waveforms for each individual patient. Therefore, the following method was used: The hatch above the number 1 indicates the left CFA waveform for patient number 1 and the hatch immediately to the right indicates the right CFA waveform for patient number 1 . The hatch above the number 2 indicates the left CFA waveform for patient number 2 and the hatch immediately to the right indicates the right CFA waveform for patient number 2 and so forth for the total number of patients in the group under investigation. This method of labelling is used for all the index plots throughout Chapters 4 and 6.

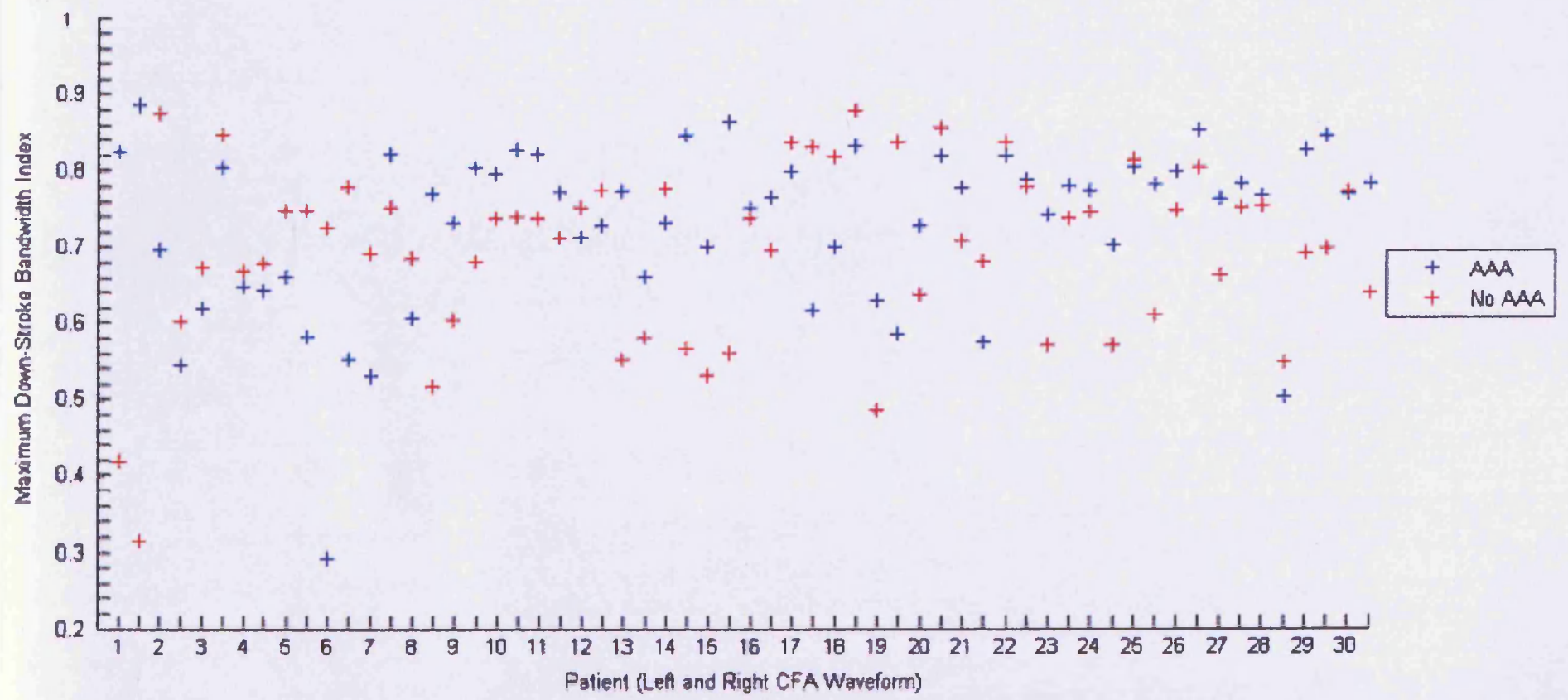

Figure A1: Axes label example for the Maximum Down-Stroke Bandwidth Index for the 30 AAA and 30 normal patients in the no significant atherosclerotic disease study groups. 